\title{
Ultraschall 2017
}

41. Dreiländertreffen ÖGUM, DEGUM, SGUM

Datum/Ort:

11.-13. Oktober 2017, Linz
Tagungspräsident:

Prim. Priv.-Doz. Dr. Wolfgang Arzt

S2 V 7: Abdomen

S6 V 1: Gefäßdiagnostik

S8 V 3: Gyn/Pränatal I

S11 V 5: Gyn/Pränatal II

S15 V 6: Gyn/Pränatal III

S18 V 11: Gyn/Pränatal IV

S22 V 13: Kontrastmittel

S24 V 8: Kopf/Hals

S27 V 10: MSK/Nervensonografie

S29 V 12: Echo/Thorax/Notfall

S35 V 2: Pädiatrie I

S37 V 9: Pädiatrie II

S40 V 4: Technik

S43 P 1: Poster Abdomen

S46 P 2: Poster Interdisziplinär

S51 P 3: Poster Gyn/Geburtshilfe

S63 Namenverzeichnis 


\section{7: Abdomen}

\section{F 3 Verlorene Gallensteine als Ursache für fremdkörperinduzierte postoperative Abszessbildung}

Autoren Gittinger $\mathrm{FS}^{1}$, Ripoll $\mathrm{C}^{2}$

Institut 1 Universitätsklinik Halle (Saale), Halle (Saale)/DE; 2 Universitätsklinikum Halle (Saale), Halle (Saale)/DE

DOI 10.1055/s-0037-1606866

Patienten und Methode Bei einem 52-jährigen Patienten war eine laparoskopische Cholezystektomie bei Gallenblasenempyem durchgeführt worden, intraoperativ fand eine Eröffnung der Gallenblase mit Eiteraustritt statt und postoperativ war es zu einer Cysticusinsuffizienz mit Notwendigkeit einer operativen Revision gekommen. Fast ein Jahr später klagte der Patient über neu aufgetretene Fieberattacken sowie rechtsseitige Oberbauchschmerzen, laborchemisch waren die Entzündungswerte erhöht. Eine daraufhin durchgeführte Schnittbildgebung erbrachte eine unklare, möglicherweise auch maligne Raumforderung des rechten Leberlappens mit Infiltration der Umgebung. Nativ-sonografisch zeigte sich eine inhomogen echoarme, infiltrativ wachsende, zur Niere hin fistulierende Raumforderung Lebersegment 5/6 mit zentralem schallschattengebendem Fremdkörper, die CEUS sprach für eine Entzündung in diesem Bereich. Eine sonografisch gesteuerte Herdbiopsie untermauerte den Verdacht auf ein entzündliches Geschehen ohne Hinweise für Malignität. Bei vermuteter Entzündung wurde eine längerfristige Antibiotikagabe eingeleitet, welche zwar unter der Dauer der Einnahme zu einer Besserung der klinischen Symptome führte, eine eigentliche Befundänderung aber nicht zu verzeichnen war. Bei Persistenz des Befundes sowie Rezidiv der klinischen Beschwerden nach Absetzen der Antibiotika wurde unter dem Verdacht auf einen Fremdkörper mit Abszessbildung eine operative Sanierung durchgeführt. Dabei erfolgte eine Lebersegmentresektion und Resektion des lateralen Zwerchfells und perirenalen Fettgewebes bei entzündlichem Konglomerattumor mit intraperitonealen Gallensteinen als auslösende Ursache.

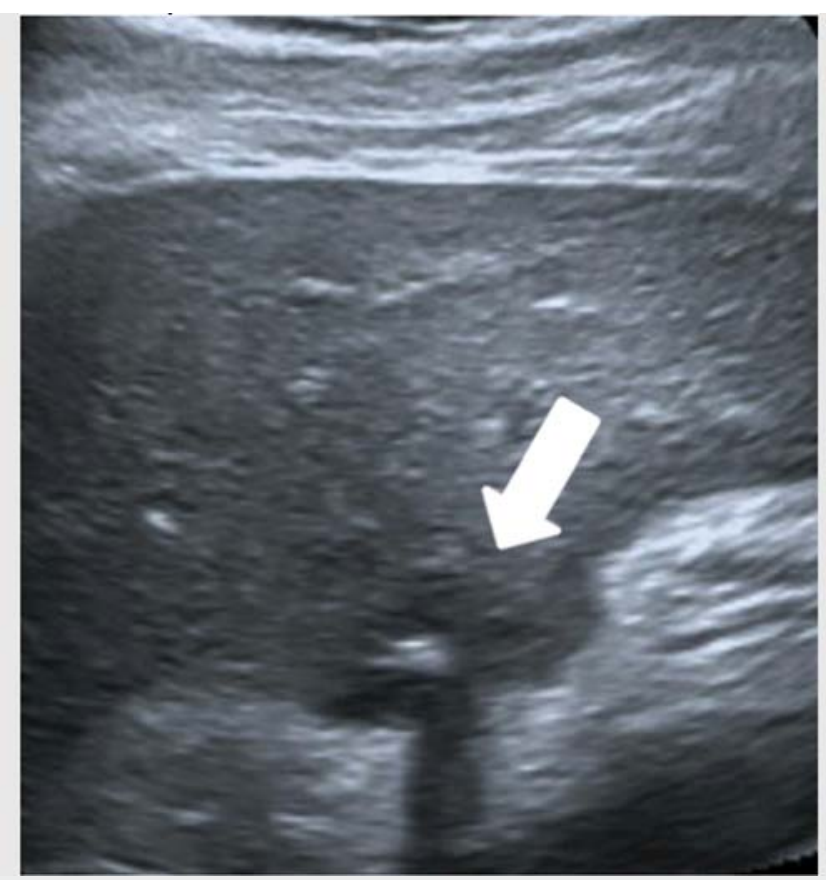

Abb. 1 Nativ-sonografischer Befund

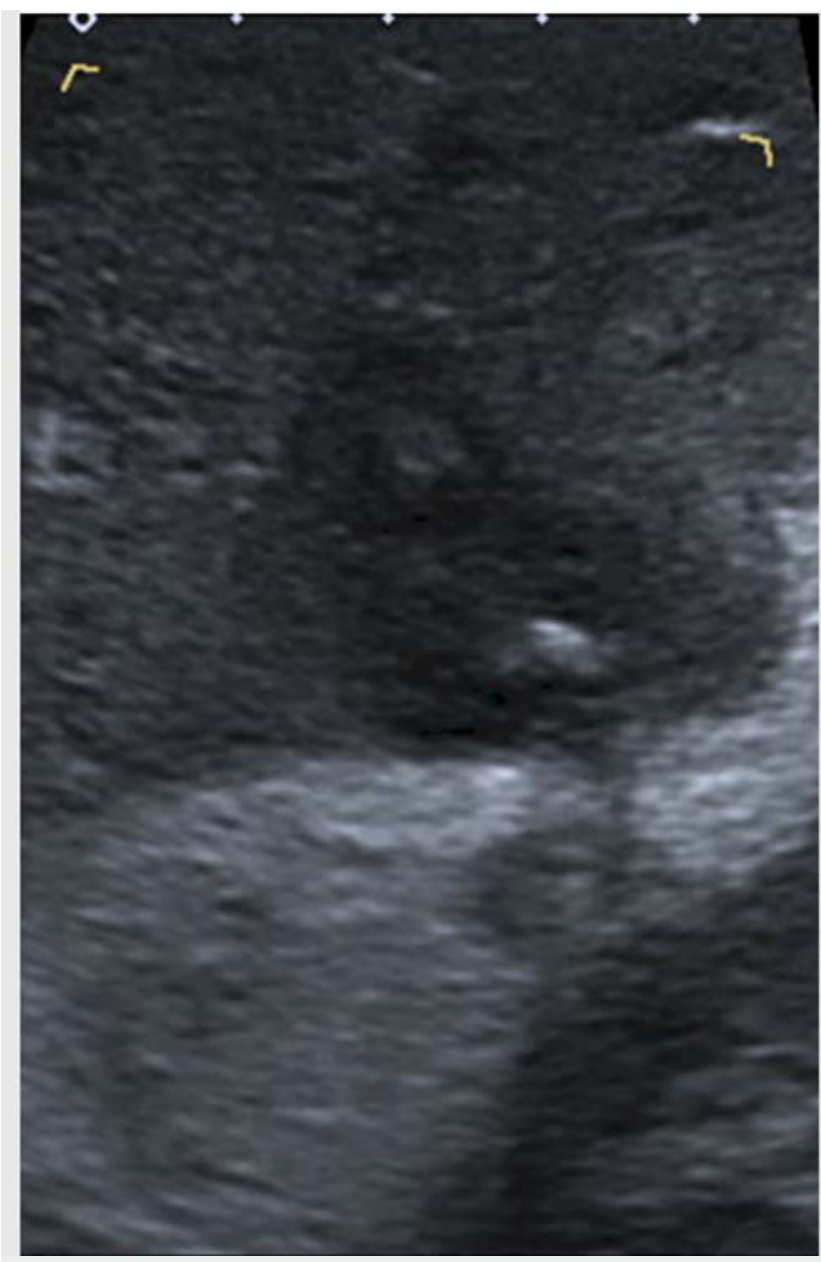

Abb. 2 Nativ-sonografischer Befund

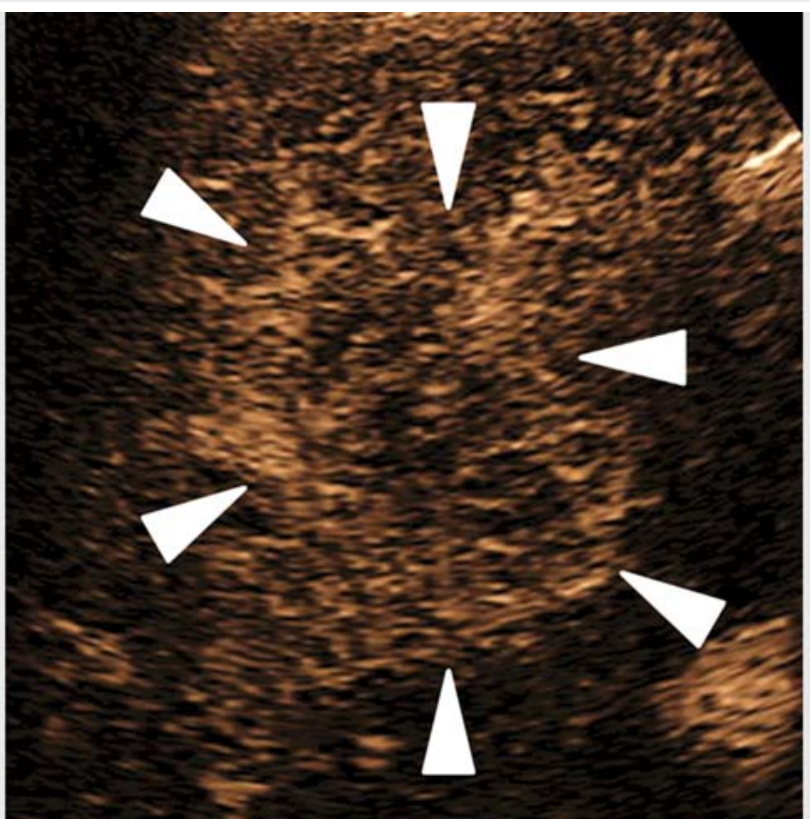

Abb. 3 CEUS, arterielle Phase

Schlussfolgerung Intra- oder perioperativ verlorene Gallensteine können auch noch längere Zeit nach erfolgter Operation ursächlich für eine fremdkörperinduzierte Abszessbildung sein. 


\section{V7.001 Stellenwert der Abdomen-Sonografie zur Fokussuche bei Patienten ohne abdominelle Symptomatik}

\author{
Autoren Kirchner $\mathrm{Gl}^{1}$, Brünn $\mathrm{K}^{1}$, Dechant $\mathrm{FX}^{1}$, Gelbrich $\mathrm{T}^{1}$, Jung $\mathrm{EM}^{1}$ \\ Institut 1 Uniklinikum Regensburg, Regensburg/DE \\ DOI 10.1055/s-0037-1606867
}

Problemstellung Bei Patienten mit Fieber und/oder erhöhten Infektwerten ohne abdominelle Symptomatik wird häufig einen Abdomen-Sonografie veranlasst, aus Sorge einen möglichen Infektfocus zu übersehen.

Ziel unserer Studie war es, den zusätzlichen Informationsgewinn der Abdomen-Sonografie bezüglich der Abklärung von Infektfoci bei Patienten ohne abdominelle Symptomatik zu untersuchen.

Patienten und Methode Retrospektiv wurden die Daten von 30 Pat. ( $m=18$, $\mathrm{w}=12$ ), die im Zeitraum von 10/16-12/16 eine Abdomen-Sonografie zur Fokussuche ohne bestehende abdominelle Symptomatik erhielten, ausgewertet. Analysiert wurden Laborwerte (CRP, Procalcitonin und Leukozyten), Vorliegen von Fieber, Abdomen-Sonografie-Befunde und die Arztbriefe. Die Abdomen-Sonografien wurden von erfahrenen Ärzten an High-End-Sonografiegeräten durchgeführt. Das Patientenalter zum Zeitpunkt der Untersuchung betrug 66+18 Jahre (MW+SD). 7 von 30 Patienten hatten alleinig eine Erhöhung der Entzündungswerte, während 23 von 30 Patienten zusätzlich Fieber hatten.

Ergebnis Bei 27 von 30 Patienten wurden keine Infektfoci in der AbdomenSonografie gefunden. Bei diesen 27 Patienten lagen folgende akute Erkrankungen vor: Pneumonie $(n=9)$, Fieber mit unklarem Infektfokus $(n=6)$, Harnwegsinfekt ( $n=5)$, CRP-Erhöhung ohne Fokus $(n=3)$, Dengue-Fieber $(n=1)$, Vaskulitis $(n=1)$, Reizung der Bursa omentalis $(n=1)$ und Endokarditis $(n=1)$. Sonografisch wurden folgende Pathologien gefunden, die erhöhte Infektwerte erklären könnten: Ein Patient mit gesichertem Harnswegsinfekt (Urinkultur: Klebsiellen) hatte einen Harnstau III. ' links bei liegendem DJ-Katheter. Eine Patientin mit metast. Ösophagus-CA und akuter Pneumonie hatte eine leichtgradige Cholestase ohne Bilirubinerhöhung und bedurfte keiner Intervention. Bei der dritten Patientin mit epiduralem Abszess zeigte sich ein Restharn trotz liegendem Urinkatheter.

Schlussfolgerung Die Abdomen-Sonografie zur Fokussuche bei Patienten ohne abdominelle Symptomatik bringt nur in seltenen Fällen einen zusätzlichen Hinweis auf einen Infektfokus.

\section{V7.002 Liegt eine höhere Prävalenz von autoimmunen Erkrankungen bei Patienten mit einer Nebenmilz vor?}

Autoren Akengin $\mathrm{A}^{1}$, Kubicka $\mathrm{S}^{1}$, Malek $\mathrm{NP}^{2}$, Boozari $\mathrm{B}^{1}$

Institut 1 Kreiskliniken Reutlingen, Reutlingen/DE; 2 Universitätsklinik

Tübingen, Tübingen/DE

DOI 10.1055/s-0037-1606868

Problemstellung Basiert auf einer klinischen Beobachtung haben wir uns die Frage gestellt, ob es eine höhere Prävalenz von autoimmunen Erkrankungen bei Patienten mit einer Nebenmilz gibt.

Patienten und Methode Zwischen den Jahren 2012 und 2015 wurden 12056 Patienten in unserer Ultraschallabteilung untersucht. Bei 235 von diesen Patienten konnte eine Nebenmilz mittels Ultraschall festgestellt werden. Die Prävalenz von autoimmunen Erkrankungen und das Risiko eine zu entwickeln wurde bei diesen Patienten retropektiv untersucht und mit einer gleich großen Kontrollgruppe aus dem gleichen Patientenkollektiv (Geschlechtsverteilung und Alter zwischen den beiden Gruppen waren ausgewogen) verglichen. Ergebnis Die Prävalenz von autoimmunen Erkrankungen war in der Nebenmilz-Gruppe mit 29,8\% (95\% Cl; 24,0 - 36,1) verglichen mit der Kontrollgruppe mit 14,9\% (95\% Cl; 10,6 - 20,1) signifikant höher ( $p<0,001)$. Patienten mit Nebenmilz haben außerdem ein höheres Risiko autoimmune Erkrankungen zu entwickeln (Prävalenzratio 2, Odds Ratio 2,42). Das Risiko ist besonders hoch bei Frauen, die älter als 50 Jahre sind (Prävalenzratio 3,49, Odds Ratio 4,48).
Diese Tatsache konnte zusätzlich durch eine Regressionsanalyse bestätigt werden ( $p<0,001$, Nebenmilz, gruppiert nach Geschlecht und Alter).

Schlussfolgerung Patienten mit Nebenmilz weisen eine höhere Prävalenz von autoimmunen Erkrankungen auf. Das Risiko für die Entwicklung einer autoimmunen Erkrankung ist insbesondere erhöht bei Frauen älter als 50 Jahre. Weitere prospektive Studien sind notwendig um diesen Zusammenhang zu bestätigen.

\section{V7.003 Intensivgallenblase - Ein ungelöstes Problem?}

Autoren Dirks $\mathrm{K}^{1}$

Institut 1 Rems-Murr-Klinikum, Winnenden/DE

DOI 10.1055/s-0037-1606869

Problemstellung Wandverdickungen der Gallenblase bei Intensivpatienten sind ein häufiges Problem, gerade beim routinemäßigen Einsatz der Sonografie als bedside Verfahren (POCUS). Frühere Arbeiten konnten zeigen, dass weniger als $20 \%$ der betroffenen Patienten eine gefürchtete acalculöse Cholezystitis haben, die mit einem hohen Perforationsrisiko einhergeht.

Patienten und Methode Anhand diverser Fallbeispiele werden Wandverdickungen der unterschiedlichsten Ursachen verglichen: Herzinsuffizienz, Schock, Sepsis, Hepatitis, ... und der fulminanten acalculösen oder calculösen Cholezystitis gegenübergestellt. Hochauflösende Sonografie, Farbdoppler und Kontrastmittel-Untersuchung werden verglichen und typische Verlaufskontrollen demonstriert.

Ergebnis Das Fehlen von Gallensteinen bei sorgfältiger Untersuchung sowie eine eher schlaffe Gallenblase sind auch auf Intensivstation wichtige B-Bild Indizien gegen eine akute Cholezystitis. Das wichtigste Zeichen der akuten Cholezystitis, der lokalisierte Druckschmerz mit dem Schallkopf, ist gerade bei beatmeten Patienten nicht überprüfbar. Die immer wieder erwähnte Dreischichtung der Gallenblasenwand ist nach unseren Erfahrungen völlig unspezifisch, gerade eine vielfache Aufsplitterung der Wandschichten spricht eher für ein Ödem als für eine Entzündung. Der Farbdoppler hilft meist wenig, am ehesten noch eine Mehrperfusion der angrenzenden Leber.

Im CEUS zeigt bei Cholezystitis die gesamte Wand ein vermehrtes Enhancement, während beim Ödem die äußeren Wandschichten kaum KM aufnehmen. Andererseits können auch gangränöse Entzündungen oder das Schockorgan GB eine Minderperfusion der Wand aufweisen. Sicher erkennbar sind Abszedierungen ins Leberbett.

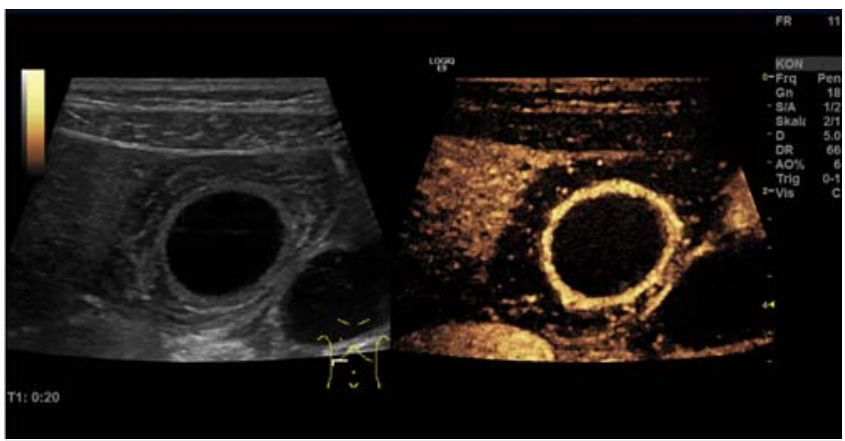

Abb. 1 GB-Wandödem bei Sepis: Enhancement nur in der Mucosa

Schlussfolgerung Diagnostik und Therapie der Intensivgallenblase bleiben schwierig: Neben einem guten klinischen Blick kann zumindest in manchen Fällen der CEUS weiterhelfen. Nur wer die Pitfalls kennt, kann oft lebensgefährlich Über- oder Unterdiagnosen vermeiden. 
V7.004 Prospektive Evaluation des Stellenwerts der ARFI-Elastografie von Leber und Milz bei Patienten nach TIPS-Anlage

Autoren Klinger $C^{1}$, Kruse $A^{1}$, Grotz $A^{1}$, Fröhlich $E^{2}$, Caca $K^{1}$, Riecken $B^{1}$ Institut 1 Klinikum Ludwigsburg, Ludwigsburg/DE; 2 Universität Tübingen, Tübingen/DE

DOI 10.1055/s-0037-1606870

Problemstellung Erste Pilotprojekte zeigten einen potenziellen Nutzen der ARFI-Elastografie von Leber und Milz in der Verlaufskontrolle von TIPS-Patienten. Ziel dieser Arbeit ist die prospektive Untersuchung des Stellenwerts der ARFI-Elastografie bei TIPS-Patienten.

Patienten und Methode In unserem Zentrum wurden von Juni 2015 bis Mai 201799 TIPS-Neuanlagen durchgeführt. Elastografische Messungen (Siemens Acuson S3000; Virtual TouchTM Tissue Quantification) von Leber und Milz erfolgten 1-5 Tage vor und 3-7 Tage nach der TIPS-Anlage (FU-1) sowie nach 3 Monaten (FU-2). Patienten mit Pfortaderthrombose, Malignomen, Tod vor dem 3-Monats-Follow-Up oder inkompletten Daten wurden ausgeschlossen. 37 Patienten wurden eingeschlossen (62,7 \pm 11,8 Jahre; 26 männlich, 11 weiblich). Indikationen zur TIPS-Anlage waren therapierefraktärer Aszites $(n=25)$, rezidivierende Varizenblutungen $(n=9)$ und hepatorenales Syndrom $(n=3)$.

Ergebnis Die präinterventionell gemessene Leber- und Milzsteifigkeit korrelierte nicht mit dem portosystemischen Drukgradienten (PPG) vor TIPS-Anlage $\left(r^{2}=0,03\right.$ bzw. $\left.r^{2}=0,05\right)$. Auch unterschieden sich die Messwerte nicht zwischen Patienten mit/ohne Aszites (3,16 vs. 3,4, $p=0,35)$, mit/ohne Varizen $(3,14$ vs. $3,24, p=0,57)$ sowie Patienten mit/ohne angiografischen Nachweis großer Umgehungskreisläufe (3,21 vs. 3,24, $p=0,84)$. Leber- und Milzsteifigkeit zeigten sowohl zum FU-1 als auch zum FU-2 keine signifikante Veränderung im Vergleich zu den präinterventionellen Messwerten (Abb. 1).

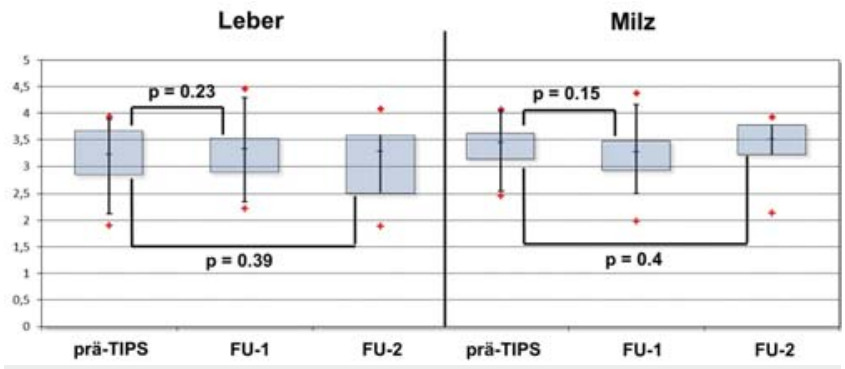

Abb. 1 ARFI-Messwerte von Leber und Milz vor TIPS-Anlage sowie 1 - 5 Tage (FU-1) bzw. 3 Monate nach TIPS-Anlage (FU-2).

Schlussfolgerung Bei ausgeprägter portaler Hypertension mit portal-hypertensiven Komplikationen korrelierten in unserem Kollektiv weder Leber- noch Milzsteifigkeit mit dem PPG. Die ARFI-Elastografie erwies sich auch in der Verlaufskontrolle nach TIPS-Anlage als nicht nützlich.

\section{V7.005 Ultraschall point Shearwave Elastografie des Pankreas: Vergleich zwischen Typ-1-Diabetikern und gesunden Probanden}

Autoren Püttmann $S^{1}$, Gräter $T^{1}$, Schmidberger $J^{1}$, Hänle $M^{1}$, Kratzer $W^{1}$ Institut 1 Universitätsklinikum Ulm, Ulm/DE

DOI 10.1055/s-0037-1606871

Problemstellung Ziel dieser Arbeit war die sono-elastografische Beurteilung des Pankreas bei Typ-1-Diabetikern im Vergleich zu gesunden Probanden.

Patienten und Methode Von 21 Patienten und 17 gesunden Probanden, die untersucht wurden, konnten 15 Patienten (10 Männer, 5 Frauen) und 15 Probanden (10 Männer, 5 Frauen) in die Pilotstudie eingeschlossen werden. Die Messungen erfolgten mittels Siemens Acuson S3000 (Siemens Healthcare,
Erlangen, Germany) mit einem 6C1-Konvexschallkopf mit der Methode der Virtual TouchTM Tissue Quantification (VTQ).

Ergebnis Die mittleren Scherwellengeschwindigkeiten lagen für das Caput des Pankreas für Patienten bei 1,0 $\pm 0,2 \mathrm{~m} / \mathrm{s}$ (Median: $1,1 \mathrm{~m} / \mathrm{s}$ ) und für Probanden ebenfalls bei $1,0 \pm 0,2 \mathrm{~m} / \mathrm{s}$ (Median: $0,9 \mathrm{~m} / \mathrm{s}$ ). Für das Corpus wurden Geschwindigkeiten von 1,2 $\pm 0,2 \mathrm{~m} / \mathrm{s}$ (Median: $1,2 \mathrm{~m} / \mathrm{s}$ ) ebenfalls für beide Gruppen gemessen. In der Pankreas Cauda konnte ein signifikanter Unterschied $(p=0,0474)$ zwischen Patienten $(1,1 \pm 0,1 \mathrm{~m} / \mathrm{s}$, Median: $1,0 \mathrm{~m} / \mathrm{s})$ und Probanden $(0,9 \pm 0,1 \mathrm{~m} / \mathrm{s}$, Median: $0,8 \mathrm{~m} / \mathrm{s})$ beobachtet werden. Im Mittelwert für das gesamte Pankreas lagen die Werte für die Patienten $(1,1 \pm 0,1 \mathrm{~m} / \mathrm{s}$, Median: $1,0 \mathrm{~m} / \mathrm{s})$ nicht signifikant $(p=0,2453)$ über den Werten der Probanden $(1,0 \pm 0,1 \mathrm{~m} / \mathrm{s}$, Median: $1,0 \mathrm{~m} / \mathrm{s})$.

Schlussfolgerung Zwischen Typ-1-Diabetikern und gesunden Probanden konnten sono-elastografisch keine Unterschiede nachgewiesen werden. Lediglich die Cauda des Pankreas zeigte bei Typ-1-Diabetikern höhere Werte. Zukünftige Studien müssen prüfen, ob dieses Ergebnis in größeren Studienpopulationen ebenfalls für die anderen Pankreasabschnitte reproduzierbar ist.

\section{V7.006 P-Shearwave ARFI-Elastografie des Pankreas bei Patienten mit Mukoviszidose - ein Vergleich mit gesunden Probanden}

Autoren Pfahler $\mathrm{M}^{1}$, Gräter $\mathrm{T}^{1}$, Schmidberger $]^{1}$, Hänle $\mathrm{M}^{1}$, Kratzer $\mathrm{W}^{1}$ Institut 1 Universitätsklinikum Ulm, Ulm/DE

DOI 10.1055/s-0037-1606872

Problemstellung Manifestationen der Mukoviszidose an der Bauchspeicheldrüse gewinnen bei den immer älter werdenden Patienten an klinischer Bedeutung. Die konventionelle Sonografie und die Acoustic-Radiation-Force-Impulse (ARFI) Bildgebung sind nicht-invasive, leicht verfügbare und einfach durchzuführende diagnostische Methoden. Das Ziel dieser Studie ist es, konventionelle sonografische Befunde und ARFI-Messwerte des Pankreas bei Patienten mit Mukoviszidose mit gesunden Kontrollpersonen zu vergleichen. Patienten und Methode 27 Patienten mit Mukoviszidose (13 Frauen/14 Männer; Durchschnittsalter 27,7 \pm 13,7 Jahre; Range 9 - 58 Jahre) und 60 gesunde Kontrollpersonen (42 Frauen/18 Männer; Durchschnittsalter 30,9 $\pm 11,0$ Jahre; Range 17 - 57 Jahre) wurden mittels der konventionellen Sonografie und der ARFI Elastografie am Pankreas untersucht.

Ergebnis Patienten mit Mukoviszidose zeigten ein echoreicheres Pankreasparenchym. Zystische Läsionen des Pankreas fanden sich bei sechs Patienten. Die ARFI Elastografie des Pankreasparenchyms ergab signifikant geringere Scherwellengeschwindigkeiten bei Mukoviszidosepatienten im Vergleich zur Kontrollgruppe $(1,01 \mathrm{~m} / \mathrm{s}$ vs. $1,33 \mathrm{~m} / \mathrm{s} ; \mathrm{p}<0,0001)$.

Schlussfolgerung Mithilfe der ARFI Elastografie stellt sich das Pankreas in vivo bei Patienten mit Mukoviszidose im Vergleich zu gesunden Kontrollpersonen deutlich weicher dar.

\section{V7.007 Veränderungen der mesenterialen Gefäße nach US-gesteuerter HIFU-Ablation beim lokal infiltrierenden Pankreasadenokarzinom}

Autoren Strunk $\mathrm{H}^{1}$, Marinova $\mathrm{M}^{1}$, Lützow $\mathrm{C}^{1}$

Institut 1 Universitätskliniken Bonn, Bonn/DE

DOI 10.1055/s-0037-1606873

Problemstellung Analyse der Auswirkungen der US-gesteuerten HIFU-Therapie auf die großen Oberbauchgefäße bei Patienten mit inoperablem Pankreaskarzinom.

Patienten und Methode Beurteilung der sonografischen und computertomografischen Untersuchungen von 50 konsekutiv mit HIFU behandelten Patienten (26 Männer und 24 Frauen) zwischen $41-82(65,0 \pm 10,2)$ Jahren vor und unmittelbar nach der HIFU-Therapie sowie nach 6 Wochen, 3 und 6 Monaten. Die Auswertung erfolgte durch zwei unabhängige Untersucher aufgeschlüsselt nach Gefäßen und Grad der Gefäßbeteiligung $(0=$ keine Gefäßbeteiligung, 
1 = Tumor reicht an das Gefäß heran, keine Einengung, 2 = mäßige Gefäßeinengung, 3 = filiforme Gefäßeinengung, 4 = Gefäßverschluss).

Ergebnis Vor HIFU-Therapie lag bei 42 der Patienten eine Beteiligung der arteriellen, 47 der venösen und 47 von beiden vor. Dabei wiesen 10 Patienten eine tumorbedingte filiforme arterielle Gefäßeinengung, drei einen Verschluss der A. mesenterica sup., fast die Hälfte $(n=24)$ einen Verschluss von V. mesenterica sup., Pfortader oder Milzvene auf. Trotz dieser Gefäßummauerungen und der zum Teil massiv erkennbaren Kollateralgefäße konnte eine HIFU-Therapie bei allen Patienten klinisch komplikationslos durchgeführt werden. Die Kontrollen unmittelbar nach durchgeführter HIFU-Therapie, zeigten bei 47 der Patienten (94\%) keine Änderung des Gefäßstatus. Durch Tumorprogress kam es bei 11 Patienten in den Verlaufskontrollen ab der sechsten Woche zu einer zunehmenden Gefäßeinengung bis hin zum Verschluss nach 6 Monaten.

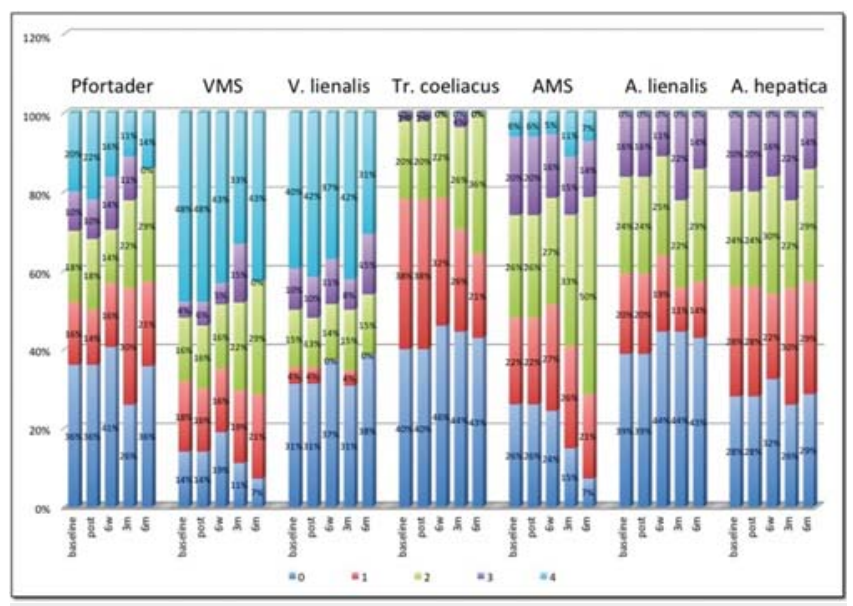

Abb. 1 Relative Zahl der Patienten ohne bzw. mit einer tumorbedingten Gefäßalteration vor (baseline) und im Verlauf direkt nach HIFU-Therapie (post), nach 6 Wochen $(6 \mathrm{w}), 3$ Monaten $(3 \mathrm{~m})$ und 6 Monaten $(6 \mathrm{~m})$ aufgeschlüsselt nach Gefäßen.

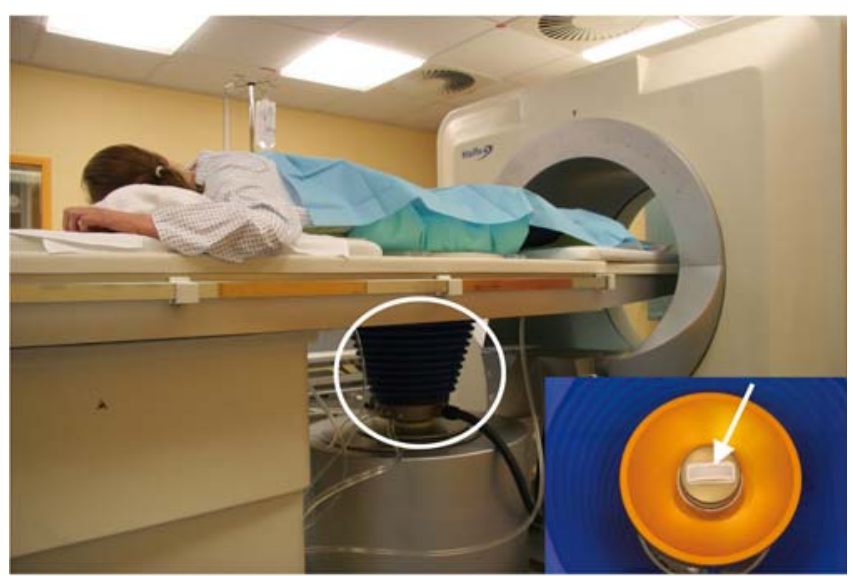

Abb. 2 Ultraschall-gesteuertes HIFU-Gerät. Der Schallkopf (Inlet: Pfeil = diagnostischer Transducer, Parabol-Wandler zur Erzeugung des therapeutischen Ultraschalls) befindet sich im Wassertank (weisses Oval) unterhalb einer Aussparung im Untersuchungstisch.

Schlussfolgerung Eine HIFU-Ablation beim lokal fortgeschrittenen Pankreaskarzinom ist auch bei mesenterialer Gefäßbeteiligung und ausgeprägten Umgehungskreisläufen sicher möglich. Im Einzelfall kann es bei bereits zuvor tumorbeteiligten Gefäßen zu einer weiteren Einengung kommen; bei den meisten Patienten (94\%) hat die Therapie aber trotz z. T. hochgradiger Stenosen keine nachteilige Auswirkung auf die Gefäßsituation.

\section{V7.008 Alveoläre Echinokokkose der Leber: Die Echinococcus multilocularis Ulm Klassifikation - Ultraschall (EMUC-US)}

Autoren Kratzer $\mathrm{W}^{1}$, Schmidberger $\mathrm{J}^{1}$, Hänle $\mathrm{M}^{1}$, Gräter $\mathrm{T}^{1}$, Grüner $\mathrm{B}^{1}$ Institut 1 Universitätsklinikum Ulm, Ulm/DE

DOI 10.1055/s-0037-1606874

Problemstellung Die Sonografie des Abdomens stellt bei Menschen mit Verdacht auf eine alveoläre Echinokkokkose (AE) eines der wichtigsten diagnostischen Instrumente dar. Ziel der Arbeit war es, eine sonografische Klassifikation für die hepatische alveoläre Echinokokkose zu entwickeln und zu etablieren.

Patienten und Methode Im Rahmen der Studie wurden retrospektiv 225 Patienten mit Leberläsionen bei alveolärer Echinokokkose beurteilt. Die sonomorphologischen Befunde wurden in ein neues Klassifikationsschema eingruppiert.

Ergebnis Die Auswertung der Ultraschallbefunde ergab, dass sich die Raumforderungen der Leber überwiegend in fünf morphologischen Ausprägungen präsentieren. Entsprechend den sonomorphologischen Strukturen wurden folgende Begrifflichkeiten etabliert: Sturm- und Hagelmuster, pseudozystisches Muster, pseudohämangiomartiges Muster, Verknöcherungsmuster und metastasenartiges Muster. Die Studie zeigt, dass das Sturm- und Hagelmuster sowie das pseudozystische Muster die am häufigsten vorkommenden Muster darstellen.

Schlussfolgerung Die Echinococcus multilocularis Ulm Klassifikation - Ultraschall (EMUC-US) ermöglicht eine Verbesserung der Diagnostik der hepatischen alveolären Echinokokkose. Die EMUC-US Klassifikation kann helfen, die unterschiedlichen klinischen Verläufe besser zu interpretieren und ermöglicht damit eine standardisierte Vergleichbarkeit von Ultraschallbefunden.

\section{V7.009 58-jähriger Patient mit Z.n. allogener Stammzelltransplantation, normaler Milzgröße und pathologischem Parenchymenhancement in der kontrastmittelunterstüzten Sonografie (CEUS): Hinweis auf funktionelle Asplenie bei normaler Milzgröße}

Autoren Trenker $C^{1}$, Wollmer $E^{2}$, Görg $C^{1}$, Neubauer $A^{2}$

Institut 1 Universitätsklinikum Marburg, Marburg/DE; 2 Universitätsklinikum Gießen und Marburg Standort Marburg und Philippsuniversität Marburg, Marburg/DE DOI 10.1055/s-0037-1606875

Patienten und Methode Ein 58-jähriger Patient mit mehrfachem Rezidiv eines Multiplen Myeloms, Z.n. allogener Stammzelltransplantation und Graft-vsHost-Disease (GvDH) wurde zur Verlaufskontrolle in unserem Interdisziplinären Ultraschallzentrum vorstellig. 2 Monate zuvor erfolgte die Einleitung einer immunsuppressiven Therapie mittels Proteinkinaseinhibitor bei ausgeprägter Haut- und Schleimhaut-GvHD. B-Bildsonografisch ist die Milz normwertig groß $(9 \times 4 \times 7 \mathrm{~cm})$, zeigt allerdings eine inhomogene Parenchymtextur. In der kontrastmittelunterstüzten Sonografie (CEUS) stellte sich früharteriell ein regelrechter arterieller Gefäßbaum dar (10s) mit anschließender homogener aber reduzierter parenchymaler Kontrastmittelanreicherung im Vergleich zum Enhancement der Leber. In der weiteren Parenchymphase (2 - 5 min) zeigte die Milz eine nahezu fehlende Kontrastmittelanreicherung. Die daraufhin initiierte Milzszintigrafie ergab die Diagnose einer Hypo-/Asplenie. Im peripheren Blutausstrich zeigte sich der Nachweis von Howell-Jolly-Körperchen als Ausdruck einer gestörten Milzfunktion.

Schlussfolgerung In der Regel ist die Milz in der CEUS durch eine starke und langandauernde Kontrastmittelanreicherung charakterisiert. Eine fehlende Milzkontrastierung wird u.a. bei Milzinfarkten und Hyposplenien (Milzgröße $<6 \times 3 \mathrm{~cm}$ ) beobachtet. Im vorliegenden Fall weist das pathologische CEUS Muster trotz normaler Milzgröße auf eine gestörte Mikrovaskularisation hin 
und damit vermutlich auch auf eine gestörte Milzfunktion. Szinitigrafisch konnte dieser Verdacht bestätigt werden. Möglicherweise stellt die CEUS eine diagnostische Methode zur Beurteilung der „funktionellen Asplenie“ dar. Eine regelrechte Größe schließt eine gestörte Funktion nichts aus.

\section{V7.010 Online-Speicherung und Publikation von Sonografiebefunden per Video-Portal}

Autoren Merkel $\mathrm{D}^{1}$, Schneider $\mathrm{C}^{1}$, Chupina $\mathrm{V}^{1}$, Kämmer J $\mathrm{C}^{2}$

Institut 1 Immanuel Klinik Rüdersdorf, Rüdersdorf bei Berlin/DE; 2 Vivantes Klinikum Kaulsdorf, Berlin/DE

DOI 10.1055/s-0037-1606876

Problemstellung Das Erkennen und Einordnen von seltenen oder gering ausgeprägten sonografischen Befunden führt regelrecht zu Unsicherheiten bei den Untersuchern.

Die Verwendung von Nachschlagewerken ist mühsam, weil die verfügbaren Bücher häufig nicht vor Ort sind, weil hier häufig kleine und teilweise veraltete Bilder abgedruckt sind und weil ein Foto nicht den Bildeindruck eines bewegten Sonografiebildes einer Real-time-Untersuchung widerspiegelt.

Patienten und Methode Mehr als 800 per Videoclip dokumentierte Sonografie-Befunde aus 3 verschiedenen Krankenhäusern werden anonymisiert und auf eine Länge zwischen 10 und 25 s geschnitten. Es erfolgen die kritische Durchsicht der Befunde mit Benennung einer Hauptdiagnose sowie die Zuordnung zu Organsystemen und zu verschiedenen Stichwörtern.

Das System ist erweiterbar und lebt von der Zuarbeit sonografisch tätiger Ärzte, die typische und/oder seltene Befunde per Videoclip aufzeichnen und zur online-Veröffentlichung zur Verfügung stellen.

Ergebnis Es entsteht eine online-Portal mit aktuell mehr als 800 Videosequenzen von typischen, anschaulichen oder auch seltenen sonografischen Befunden.

Das Portal ist frei über das Internet erreichbar, das Design ist für die Nutzung an mobilen Endgeräten optimiert. Ein schneller Internetzugang ist zum reibungsfreien Aufrufen der Videos von Vorteil.

Das Auffinden der relevanten Befunde erfolgt entweder über ein Stichwortsystem oder durch eine diagnosebasierte Suchfunktion.

Schlussfolgerung Durch das Videoportal ist eine ubiquitär verfügbare Plattform entstanden, mit deren Hilfe differentialdiagnostische Fragestellungen von typischen, untypischen und vor allem von seltenen Sonografiebefunden im bewegten Bild geklärt bzw. mit den selbst erhobenen Befunden abgeglichen werden können.

Das System ist frei zugänglich und lädt zum Hochladen eigener Videos ein, welche nach einer fachlichen Überprüfung eine permanente Aktualisierung und Erweiterung dieser umfangreichen Befundsammlung ermöglicht.

\section{V7.011 Qualität der B-Bild-Sonografie auf modernen High-End-Geräten - eine verblindete Studie}

Autoren Merkel $D^{1}$, Chupina $V^{1}$, Schneider $C^{1}$

Institut 1 Immanuel Klinik Rüdersdorf, Rüdersdorf bei Berlin/DE

DOI 10.1055/s-0037-1606877

Problemstellung Für eine aussagefähige abdominelle Sonografie ist die technische Qualität des B-Bildes maßgeblich. Insbesondere zum sonografischen Nachweis kleiner parenchymatöser Läsionen in Leber oder Pankreas werden hohe technische Anforderungen an das Sonografiegerät gestellt.

Patienten und Methode Zwischen Januar und Mai dieses Jahres testeten wir die B-Bild-Qualität bei Sonografiegeräten von insgesamt 7 in Deutschland konkurrierenden Geräteherstellern. Dabei griffen wir auf die jeweils neuesten Geräte aus dem High-End-Sektor mit Listenpreisen von mehr als 50 T€ zurück. Hierfür erhielten 4 gesunde Probanden Ulltraschalluntersuchungen mit jedem der 7 untersuchten Ultraschallgeräte in 4 definierten abdominellen Standardschnitten. Die Dokumentation erfolgte digital durch Aufzeichnung von Videosequenzen. Die so entstandenen 112 Videosequenzen wurden bezüglich jeg- licher Herstellerangaben anonymisiert. Schließlich erfolgte das paarweise $\mathrm{Ne}$ beneinanderstellen von jeweils zwei korrespondierenden Sequenzen.

Alle so entstandenen 336 Videopärchen wurden von insgesamt 8 erfahrenen Ultraschalluntersuchern hinsichtlich technischer Bildqualität im direkten visuellen Vergleich bewertet.

Ergebnis Mit dieser systematischen Untersuchung kristallisierten sich 2 der 7 untersuchten Geräte als Favoriten in Qualität der B-Bild-Darstellung heraus. Die Rangfolge der untersuchten Geräte korrelierte weder mit dem empfohlenen Verkaufspreis noch mit der Marktpräsenz der Gerätehersteller.

Schlussfolgerung Bei der Auswahl eines neuen High-End-Sonografiegerätes für eine gastroenterologische Klinik spielen viele unterschiedliche Kriterien eine Rolle. Weder ein besonders teures Gerät noch eine hohe Markpräsenz der Herstellerfirma waren eine Garantie für eine optimale B-Bild-Qualität.

\section{1: Gefäßdiagnostik}

\section{F 1 Fetale hepatische AV-Malformationen - seltene deletäre Gefäßanomalien - 2 pränatale Fallberichte}

Autoren Weichert $\mathrm{J}^{1}$, Gembicki $\mathrm{M}^{1}$, Hartge $\mathrm{D}^{1}$

Institut 1 Universitätsklinikum Schleswig-Holstein, Lübeck/DE

DOI 10.1055/s-0037-1606878

Patienten und Methode Arteriovenöse hepatische Gefäßanomalien sind seltene Fehlbildungen, die pränatal häufig zu einer frühen kardialen Kompromittierung des Feten führen (high cardiac output failure).

Wir berichten über 2 pränatale Fälle einer intrahepatischen AV-Malformation mit atypischer arterieller Gefäßverbindung des intrahepatischen Anteils der Vena umbilikalis und des Truncus coeliacus. Diagnosestellung im 1. Fall in der 11+6. SSW bei Hydrops fetalis. Farbdopplersonografisch zeigte sich ein vom Truncus coeliacus zur vorderen Bauchwand ziehendes breites aberrantes Gefäß, welches dann in die UV einmündete (Abb. 1, Vmax >120 cm/s). Echokardiografisch holosystolische Triskupidalinsuffizienz, ansonsten zeigte der Fet keine weiteren strukturellen Auffälligkeiten. Eine Karyotypisierung ergab eine Trisomie $21(47, X X,+21)$. Die Eltern hatten sich für eine SS-Beendigung entschieden.

Im Gegensatz hierzu fiel im 2. Fall einer diskordant wachsenden di-di Geminigravidität in der 20+5. SSW eine Gefäßanomalie zw. Aorta und subphrenisch zur UV ziehend auf (Abb. 2, Vmax $>100 \mathrm{~cm} / \mathrm{s}$ ), Ductus venosus hoch pulsatil, echokardiografisch beschleunigter laminarer Flow in der Aorta ascendens sowie Kardiomegalie. Die Eltern entschieden sich für die Fortführung der SS, bei progredienten Dopplerauffälligkeiten primäre Sectio in der 30+1. SSW. Der dystrophe Fet zeigte ein schweres Atemnotsyndrom und kardiorespiratorische Instabilität. Am 2. postpartalen Tag kam es zu einer fulminanten NEC (infolge chonischer Unterversorgung der unteren Körperregion) und Exitus letalis am selben Tag. Der Karyotyp war unauffällig 46,XY.

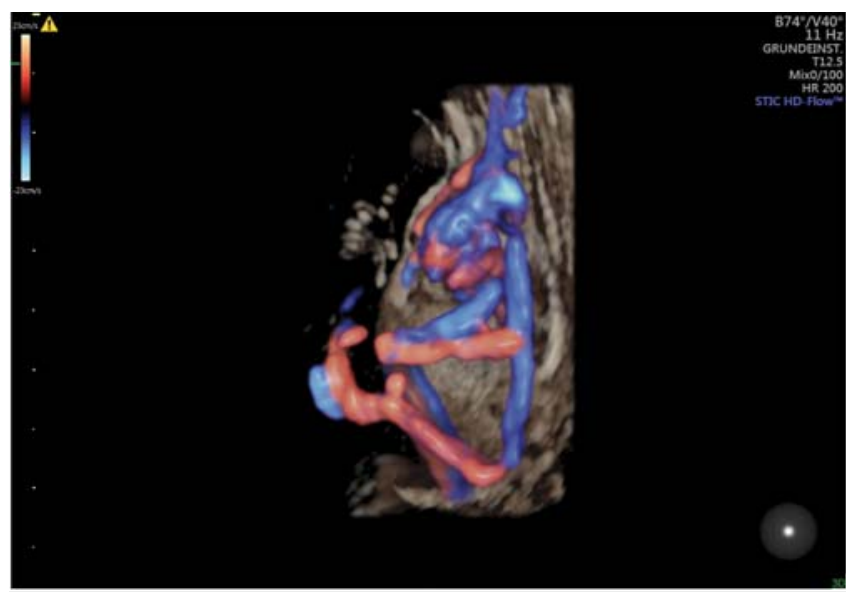

Abb. 1 


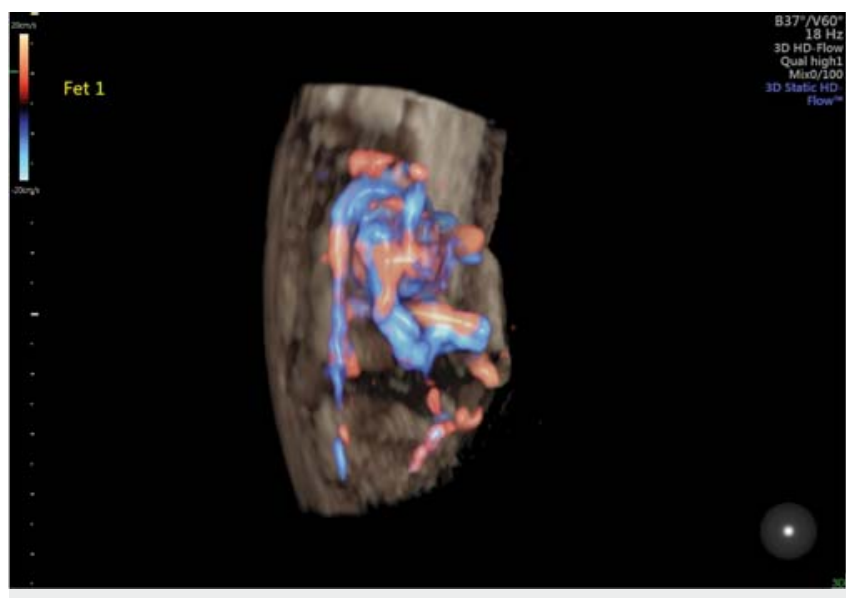

Abb. 2

Schlussfolgerung Die dopplersonografisch gut zu stellende Diagnose einer intrahepatischen AVM sollte eine ausführliche interdisziplinäre Beratung (unter Berücksichitgung der hohen prä- und perinatalen Mortalitätsrate) zur Folge haben. Eine Karyotypisierung ist dringend zu empfehlen. Verbindliche Empfehlungen hinsichtlich möglicher therapeutischen Ansätze fehlen bis dato.

\section{V1.001 Kontrastmittelsonografie in der Aktivitätsbeurteilung der Takayasu-Arteriitis}

Autoren Lottspeich $C^{1}$, Schröttle $A^{1}$, Dechant $C^{1}$, Schulze-Koops $H^{1}$, Hoffmann $\mathrm{U}^{1}$, Czihal $\mathrm{M}^{\top}$

Institut 1 Klinikum der Ludwig-Maximilians-Universität, München/DE DOI 10.1055/s-0037-1606879

Problemstellung Der potentielle Stellenwert der hochauflösenden Kontrastmittelsonografie (CEUS) in der Aktivitätsbeurteilung der Takayasu-Arteriitis soll evaluiert werden.

Patienten und Methode Seit 2016 wurden Patientinnen mit Takayasu-Arteriitis, die zusätzlich zur Farbduplexsonografie eine CEUS-Untersuchung der supraaortalen Arterien erhielten, in diese retrospektive Pilotstudie eingeschlossen. Voraussetzung für den Studieneinschluss war eine vaskulitistypische Verdickung des Intima-Media-Komplexes $>1,5 \mathrm{~mm}$ in mindestens einem Segment der supraaortalen Arterien (A. carotis communis, A. subclavia/axillaris) in der Farbduplexsonografie. Die klinische Aktivität wurde auf Basis von Anamnese, klinischer Untersuchung, humoralen Entzündungszeichen im Labor und Befunden der konventionellen Gefäßbildgebung (Duplexsonografie und/oder Schnittbildgebung) eingeschätzt und anhand etablierter Score-Systeme ( $\mathrm{NIH}-$ Score, ITAS-A) determiniert. Das Segment der supraaortalen Arterien mit der ausgeprägtesten Verdickung des Intima-Media-Komplexes wurde mittels hochauflösender Kontrastmittelsonografie untersucht (GE Logiq E9, Breitbandlinearschallkopf $6-15 \mathrm{MHz}, 1,5 \mathrm{ml}$ Sonovue ${ }^{\circledR}$ intravenös unter standardisierten Geräteeinstellungen). Der Übertritt von Kontrastmittelbläschen in die Gefäßwand wurde visuell beurteilt ( $\mathrm{rrad} 0=$ keine/minimale Anreicherung, Grad 1 = moderate Anreicherung, Grad 2 = starke Anreicherung).

Ergebnis 12 Untersuchungen (A. carotis communis: $n=9$, A. subclavia/axillaris: $n=3$ ) bei 8 Patientinnen wurden in die Analyse einbezogen. Zum Zeitpunkt der Ultraschalluntersuchung wurde die Erkrankung als klinisch aktiv ( $\mathrm{NIH}$-Score $\geq 2)$ in 5 Fällen und inaktiv ( $\mathrm{NIH}$-Score $\leq 1)$ in 7 Fällen beurteilt. Die mittlere Wanddicke der kontrastmittelsonografisch untersuchten Segmente betrug 2,5 mm (1,7-4,0 mm). Eine Kontrastmittelanreicherung Grad $1 / 2$ lag bei $100 \%$ der klinisch aktiven und $14 \%$ der klinisch inaktiven Fälle vor (Sensitivität 100\%, Spezifität 86\%).

Schlussfolgerung Die Kontrastmittelsonografie könnte einen diagnostischen Stellenwert in der bildgebenden Verlaufsbeurteilung der Takayasu-Arteriitis erlangen.

\section{V1.002 Mittels B-Flow können über die vergleichende Querschnittsflächenbestimmung $\mathrm{ACI}$ Stenosen verlässlich eingeschätzt werden}

Autoren Ausmeier $\mathrm{A}^{1}$, Weskott $\mathrm{HP}^{2}$

Institut 1 Nordstadt Klinikum, Hannover/DE; 2 Klinikum Siloah, Hannover/DE DOI 10.1055/s-0037-1606880

Problemstellung B-Flow stellt über ein Subtraktionsverfahren eine dopplerunabhängige Technik zur Berechnung von Stenosegraden der ACI dar. Die morphologisch ermittelten intra- und poststenotischen Flächenwerte wurden mit den dopplersonografisch gemessenen maximalen systolischen Geschwindigkeitswerten korreliert.

Patienten und Methode Eingeschlossen wurden 36 Männer und 16 Frauen mit ACl Stenosen zwischen $43 \%$ und $92 \%$.

Um den Stenosegrad nach NASCET zu definieren, wurde die Fläche der ACl auf der stenosierten Seite mit der distalen Referenzfläche korreliert. In gleicher Sitzung wurden die maximalen systolischen Geschwindigkeiten mittels PWDopplerverfahren bestimmt. Alle Berechnungen erfolgten dreimal.

US Gerät: LOGIQ E9,9L (GE Healthcare, Milwaukee, WI, USA).

Ergebnis Das Durchschnittsalter in der Gruppe der Männer betrug 71,2+7,9 Jahre, in der Gruppe der Frauen 67,8+7,7 Jahre. Die Stenosegrade ergaben bei den Männern 46-92\% bei maximalen systolischen Geschwindigkeiten von 0,99 - 5,64 mm/s und bei den Frauen 42,6 - 87,7\% bei systolischen Geschwindigkeiten von $0,76-4,25 \mathrm{~mm} / \mathrm{s}$.

Für die ACl-Stenosen betrug die Korrelation zwischen der Stenosefläche und systolischer Geschwindigkeit $r=0,65$ bei Männern und $r=0,72$ bei Frauen. Wegen starker Verkalkung konnte bei $22 \%$ der Patienten mit hochgradiger Stenose die Stenosefläche nicht bestimmt werden. Im Vergleich zum Nomogramm Kollektiv entspricht eine $>70 \%$ ige Stenose einer Flächenreduktion von $<0,054 \mathrm{~cm}^{2}$ bei Frauen und $<0,068 \mathrm{~cm}^{2}$ bei Männern.

Schlussfolgerung Es fand sich eine gute Korrelation zwischen hämodynamischer und morphologischer Stenosegraduierung.

Das B-Flow-Verfahren eignet sich als additives Verfahren bei der Einschätzung von ACl Stenosen, v.a. bei Tandem-, vorgeschalteten z. B. Aortenklappenstenosen oder nicht darstellbarem Referenzsegment.

\section{V1.003 „Post-Exercise Pulsatility Index“ weist Behandlungseffekte bei peripherer arterieller Verschlusskrankheit (pAVK) aus}

Autoren Udelnow $\mathrm{A}^{1}$, Hawemann $\mathrm{M}^{2}$, Kropf $\mathrm{S}^{3}$, Friebe $\mathrm{B}^{1}$, Meyer $\mathrm{F}^{1}$, Halloul $Z^{1}$

Institut 1 Uniklinik Magdeburg, Magdeburg/DE; 2 Universität Magdeburg, Madgeburg/DE; 3 Universität Magdeburg, Magdeburg/DE

DOI 10.1055/s-0037-1606881

Problemstellung Hämodynamische Parameter (HP) haben sich noch nicht als entscheidende diagnostische Kriterien bei der peripheren arteriellen Verschlusskrankheit (pAVK) etablieren können.

Hypothese der Untersuchung war es, dass mittels „Post-exercise“-Messungen pAVK-Patienten besser von gesunden Personen („healthy persons“ - HP) unterscheiden und dass diese Messungen hämodynamische Verbesserungen nach Behandlungsmaßnahmen detektieren.

Patienten und Methode 19 gesunde Voluntäre (HP) und 23 konsekutive pAVK-Patienten (Stadium IIb bis IV nach Fontaine) nahmen an der Untersuchung teil. Alle Teilnehmer wurden Messungen von „peak systolic velocity“ (PSV), „end-diastolic velocity“ (EDV), „minimal diastolic velocity“ (MD), „timeaveraged maximal velocities“ (TAMAX), Resistenzindex (RI) und Pulsatilitätsindex (PI) vor und nach standardisierten Bewegungsübungen (nach 1, 2, 3, 4 und 5 Minute[n] nach Übungsende) unterzogen. Die pAVK-Patienten wurden zusätzlich vor und nach Behandlungsmaßnahmen untersucht.

Ergebnis Im Vergleich der Ruhewerte waren HP und pAVK nicht signifikant unterschiedlich. PSV erhöhte sich nach Behandlung bei pAVK um $5 \mathrm{~cm} / \mathrm{s}$ („paired t-test“: p 0,025) im Vergleich zu vor der Behandlung. Die „areas under the 
curve of the receiver-operating-curves“ (AUC of ROC curves) für die Differenzierung von HP von pAVK-Patienten war 0,72 für TAMAX, 0,62 für PI und 0,57 für PSV. Im Zeitverlauf nach Bewegungsübungen durch wiederholte Messungen ANOVA zeigte sich, dass HP sich signifikant in PI, RI und PSV von pAVKPatienten vor und nach der Behandlung unterschieden ( $p<0,001$ für jeden Parameter). Bei pAVK-Patienten wurde ein signifikant verbesserter PI nach Behandlung im Vergleich zu prätherapeutischen Werten gefunden (p: 0,042). Schlussfolgerung HP können nicht besonders gut von pAVK-Patienten durch hämodynamische Parameter in Ruhe unterschieden werden, jedoch weit besser durch charakteristische Veränderungen nach standardisierter Bewegungsübung.

\section{V1.004 FKDS-Nachweis von Gefäßmalformationen bei seltenen Hemihyperplasie-Syndromen}

Autoren Urban $\mathrm{P}^{1}$, Tinschert $\mathrm{S}^{2}$

Institut 1 Evangelische Elisabeth Klinik, Berlin/DE; 2 Medizinische Universität, Innsbruck/AT

DOI 10.1055/s-0037-1606882

Patienten und Methode Wir haben 72 Patienten mit seltenen regionalen Riesenwuchs Syndromen klinisch und mittels farbkodierter Duplexsonografie (FKDS), hinsichtlich der Qualität malformierter Gefäße untersucht.

Ergebnis Nur bei 3 Patienten trafen die aktuell gültigen Kriterien des ProteusSyndroms zu. 24 konnten als CLOVES-Syndrom klassifiziert werden, 11 zeigten die Charakteristika einer Hemihyperplasie-multiple Lipomatose, 4 Patienten die eines Macrocephalie-kapilläres Teleangiektasie Syndroms, bei 2 Patienten wurde ein Cowden (SOLAMEN)-Syndrom diagnostiziert. 4 Patienten mit Sturge-Weber-Syndrom hatten zusätzliche Hemihyperplasien. 3 Patienten ohne Gefäßveränderungen zeigten eine Makrodaktylie.

Die verbliebenen 21 Patienten wiesen nicht klassifizierbare Fehlbildungen auf. Hiervon fanden sich bei 9 Patienten Kombinationen aus vaskulären Malformationen mit nicht lipomatösen Hemihyperplasien, bei 3 Patienten eine Kombination aus vaskulärer Malformation mit Lipomatose. 4 Patienten waren durch Gigantismus, 5 durch alleinige Lipomatose charakterisiert.

Insgesamt fanden wir bei 16 Patienten keinen Hinweis auf malformierte Gefäße.

In den anderen Gruppen zeigten die vaskulären Anomalien ein weites Spektrum, bis hin zu ausgedehnten Kombinationen aus venösen, lymphatischen und kapillären Läsionen.

Obwohl die Inzidenz ausgedehnter trunkulärer Malformationen bei schwerer Hyperplasie größer war, fanden wir keine strenge Korrelation zwischen dem Ausmaß der Hyperplasie und dem Ausmaß vaskulärer Malformationen.

Die Gruppe der Patienten mit CLOVES-Syndrom wies das breiteste Spektrum vaskulärer Anomalien, mit größter Anzahl lymphatischer Malformationen auf. Bei 4 Patienten von diesen und einem Proteus-Patienten detektierten wir zusätzliche Malformationen im intra- und/oder retroperitonealen Gefäßsystem.

Schlussfolgerung Bei Patienten mit regionalem Riesenwuchs lassen sich durch systematische FKDS-Untersuchungen in großer Anzahl vaskuläre Beteiligungen nachweisen.

\section{3: Gyn/Pränatal I}

\section{V3.001 Pulmonalatresie mit intaktem Ventrikelseptum - Vergleich des Outcomes bei pränataler vs. postnataler Diagnose}

Autoren Wolter $A^{1}$, Enzensberger $C^{1}$, Kawecki $A^{1}$, Degenhardt $]^{2}$, Stressig $R^{2}$, Ritgen $J^{2}$, Thul $J^{3}$, Axt-Fliedner $R^{4}$

Institut 1 Zentrum für Frauenheilkunde und Geburtshilfe,

Universitätsklinikum Gießen \& Marburg, Gießen/DE; 2 praenatalplus.de, Köln/ DE; 3 Klinik für Kinderkardiologie und angeborene Herzfehler,
Universitätsklinikum Gießen \& Marburg, Gießen/DE; 4 Universitätsklinikum Gießen und Marburg, Standort Gießen, Gießen/DE

DOI 10.1055/s-0037-1606883

Problemstellung Ziel unserer retrospektiven Auswertung war der perinatale Outcome-Vergleich von Patienten mit pränatal diagnostizierter Pulmonalatresie mit intaktem Ventrikelseptum (PAIVS) versus Patienten mit postnataler Diagnosestellung.

Patienten und Methode Ausgewertet wurden 47 Patienten mit PAIVS, die zwischen 01/2008 und 12/2016 in unserem Kinderherzzentrum, unserer Pränataldiagnostik oder in der Praxis praenatal.de in Köln vorstellig waren.

Ergebnis Bei 44/47 (93,6\%) Patienten war die Diagnose PAIVS pränatal bekannt, in $3 / 47(6,4 \%)$ der Fälle, welche in das Kinderherzzentrum verlegt wurden, wurde die Diagnose postpartal gestellt.

$6 / 44(13,6 \%)$ Patienten mit pränataler Diagnose sind pränatal lost to follow up, 1 (2,3\%) Schwangerschaftsabbruch wurde durchgeführt und $2(4,6 \%)$ intrauterine Fruchttode traten auf. 35/44 (79,5\%) Patienten wurden lebend geboren. 3 (8,6\%) waren lost to follow up < 30 Tage postpartum. Das mittlere follow up überlebender Patienten betrug 837 Tage.

9/35 (25,7\%) Patienten verstarben: $6 / 9$ (66,7\%) verstarben innerhalb 30 Tage postpartum, 2/9 (22,2\%) verstarben vor einer Intervention/Operation. 2/9 $(22,2 \%)$ hatten extracardiale Fehlbildungen.

$1 / 3(33,3 \%)$ Patienten mit postnataler Diagnose verstarben innerhalb der ersten 30 Tage postpartal nach Katheterintervention.

Schlussfolgerung Die Sterberate an Patienten mit PAIVS ist mit 25,7\% hoch, besonders innerhalb der ersten 30 Tage postpartal. Es konnten keine signifikanten Unterschiede bezüglich der Überlebensrate zwischen Patienten mit prä- und postnataler Diagnose bestimmt werden.

\section{V3.002 Absent pulmonary valve syndrome - Diagnose, Spektrum und Outcome in 71 pränatal diagnostizierten Fällen}

Autoren Axt-Fliedner $\mathrm{R}^{1}$, Kurkevych $\mathrm{A}^{2}$, Słodki $\mathrm{M}^{3}$, Respondek-Liberska $\mathrm{M}^{3}$, Zych-Krekora $K^{3}$, Stressig $R^{4}$, Ritgen $J^{4}$, Rizzo $G^{5}$, Krapp $M^{6}$, de Catte $L^{7}$, Mielke $G^{8}$, Bosselmann $S^{8}$, Meyer-Wittkopf $M^{9}$, Kawecki $A^{1}$, Wolter $A^{1}$, Mamalis $\mathrm{M}^{1}$, Enzensberger $\mathrm{C}^{1}$

Institut 1 Zentrum für Frauenheilkunde und Geburtshilfe, Universitätsklinikum Gießen \& Marburg, Gießen/DE; 2 Fetal Cardiology Unit, Ukrainian Children's Cardiac Center, Kyiv/UA; 3 Department of Prenatal Cardiology, Polish Mother's Memorial Hospital Research Institute, Lodz/PL; 4 praenatalplus.de, Köln/DE; 5 Department Obstetrics and Gynecology Università di Roma Tor Vergata, Roma/IT; 6 amedes experts Hamburg, Hamburg/DE; 7 Department of Obstetrics ad Gynecology, Fetal Medicine University Hospitals Leuven, Leuven/BE; 8 Pränatalzentrum Stuttgart, Stuttgart/DE; 9 Department of Gynecology and Obstetrics at the Health Center Rheine, Mathias Spital, Rheine/DE

DOI 10.1055/s-0037-1606884

Problemstellung Analyse des Spektrums und des Outcomes pränatal diagnostizierter Fälle mit absent pulmonary valve syndrome (APVS). Eingeschlossen wurden Feten sowohl mit APVS und Fallot Tetralogie (TOF/APVS), als auch mit APVS und intaktem Ventrikelseptum (APVS/IVS).

Patienten und Methode Retrospektive Multicenterstudie der International Prenatal Cardiology Collaboration Group (IPCCG). Analyse klinischer und echokardiografischer Datenbanken aus 9 Studienzentren aus dem Zeitraum 2012 2016 mit Erhebung entsprechender klinischer und echokardiografischer Parameter.

Ergebnis Insgesamt wurden 71 Fälle eingeschlossen, 59 (83,1\%) TOF/APVS und 12 (16,9\%) APVS/IVS. In 18,3\% (13/71) der Fälle wurde die Diagnose im ersten Trimenon gestellt. Es bestand eine hohe Assoziation mit Hydrops fetalis im ersten Trimenon (9/13, 69\%). Eine Chromosomenstörung lag bei $45 \%$ der Fälle mit bekanntem Karyotyp (13/29) vor, Microdeletion 22q11.2 $(n=6$, 
21\%) war die häufigste Aneuploidie. Ein intrauteriner Fruchttod trat in 14,3\% $(9 / 63)$ der Fälle mit bekanntem Outcome auf.

Overall survival nach Diagnosestellung betrug 28,1\% (20/71), 28,8\% bei TOF/ APVS und $25 \%$ bei APVS/IVS. Survival to birth lag bei $50 \%$ (27/54) für TOF/ APVS und 44,4\% (4/9) für APVS/IVS. Survival beyond neonatal period betrug $84,6 \%$ (22/26) für TOF/APVS und 100\% (3/3) für APVS/IVS.

Schlussfolgerung Die Diagnosestellung von APVS im 1. Trimenon ist möglich, das Outcome wird jedoch durch Aneuploidien, Hydrops fetalis und offenen Ductus arteriosus limitiert. Überlebensraten von $>80 \%$ können in isolierten Fällen von APVS/TOF und APVS/IVS erlangt werden.

\section{V3.003 Gewicht und Kopfumfang bei Feten mit HLH versus TGA, perinatales Management}

\author{
Autoren Janke $\mathrm{A}^{1}$, Schramm $\mathrm{T}^{1}$, Minderer $\mathrm{S}^{1}$, Gloning $\mathrm{KP}^{1}$ \\ Institut 1 Pränatal-Medizin München, München/DE \\ DOI 10.1055/s-0037-1606885
}

Problemstellung Die pränatale Wachstumsrestriktion bei Herzfehlerkindern hat über die unmittelbaren Auswirkungen der Organfehlbildung hinaus, Konsequenzen für das perinatale Management. Ziel dieser Untersuchung war eine Analyse von Wachstumsparametern (Gewicht und Kopfumfang) sowie daraus abgeleitet eine Überlegung zum optimalen Entbindungszeitpunkt.

Patienten und Methode Von 2000 bis 2017 wurden unmittelbar postnatal Körpergewicht und Kopfumfang von 54 karyotypisch und extrakardial unauffälligen Kindern mit isolierten Herzfehlern - 22 mit primärem Hypoplastischem Linksherz (HLH) und 32 mit Transposition der großen Arterien (TGA) - untersucht und mit Normperzentilen verglichen.

Ergebnis HLH-Kinder hatten zum Geburtszeitpunkt ein signifikant niedrigeres Körpergewicht (Median an der 27. Perzentile) und einen kleineren Kopfumfang (Median an der 25. Perzentile). Die TGA-Kinder zeigten dagegen ein normales Körpergewicht (Median an der 55. Perzentile) und einen leicht reduzierten Kopfumfang (Median an der 44. Perzentile). Das Gestationsalter beider Herzfehlerkohorten lag zum Entbindungszeitpunkt im Median bei $38+6$ Wochen.

Schlussfolgerung Wachstumsrestriktion ist bei Kindern mit kongenitalen Herzfehlern offenbar ein kardiospezifisches Phänomen. Sie darf nicht mit intrauteriner Wachstumsrestriktion anderer Ursache verwechselt werden, da letztere regelhaft zur vorzeitigen Beendigung der Schwangerschaft zwingt. Das postnatale kardiochirurgische Outcome von Herzfehlerkindern verbessert sich dagegen mit jeder vollendeten Schwangerschaftswoche bis zum Termin. Ziel ist es, diese Kinder nur bei zwingender fetaler oder maternaler Indikation zu entbinden. Wir empfehlen daher eine individualisierte Betrachtung und sorgfältige Überwachung dieser Risikogruppen im Perinatalzentrum.

\section{V3.004 Pränatales Management eines Feten mit HLHS mit restriktivem Foramen ovale und Hydrops fetalis mittels intrauteriner Vorhofseptumstenteinlage}

Autoren Weichert $A^{1}$, Tucher $E$ von ${ }^{1}$, Schulze $W^{1}$, Paulick $J^{2}$, Lebek $H^{2}$, Berg $C^{3}$, Henrich $W^{1}$

Institut 1 Charité - Universitätsmedizin Berlin, Berlin/DE; 2 Praxis für Pränatale Diagnostik, Berlin/DE; 3 Universitätsklinikum Bonn, Bonn/DE DOI 10.1055/s-0037-1606886

Patienten und Methode Angeborene Herzfehler gehören zu den häufigsten organspezifischen Fehlbildungen und sind die neonatale Haupttodesursache bei angeborenen Fehlbildungen. Als einer der schwersten Herzfehler gilt das hypoplastische Linksherzsyndrom (HLHS), welches ungefähr 1,6\% aller angeborenen Herzfehler ausmacht und unbehandelt in über $90 \%$ der Fälle zum Tod innerhalb der ersten 30 Lebenstage führt. Die Therapie erfolgt üblicherweise durch Operationsverfahren in drei Schritten (staged repair).

Im Rahmen des HLHS ist ein restriktives foramen ovale (FO) ein ungünstiger Prognosefaktor, der sowohl die Möglichkeit des staged repair als auch einer Herztransplantation negativ beeinflusst.
Fallreport Eine 32-jährige Patientin (V-Gravida, II-Para) wurde uns wegen eines HLHS in 24+1 SSW zugewiesen. Ursächlich war eine kritische Aortenklappenstenose mit retrograder Perfusion des Aortenbogens. Ferner bestand eine dysplastische Mitralklappe mit einer Mitralklappeninsuffizienz. Darüberhinaus zeigte sich eine Endocardfibroelastose des hypokontraktilen linken Ventrikels, eine Cardiomegalie sowie ein links > rechts Shunt über ein restriktives FO. Eine Woche später entwickelte sich ein Pericarderguss und Aszites, als Zeichen der cardialen Dekompensation. Die Schwangere wurde daraufhin zur Evaluation der Möglichkeit einer intrauterinen Aortenvalvuloplastie weiterüberwiesen.

Mangels technischer Umsetzbarkeit (Aortenwurzel 1,9 mm) wurde stattdessen ein Stent in das Vorhofseptum in 26+0 SSW eingelegt. Hiernach bildeten sich sowohl der Pericarderguss als auch der Aszites zurück.

Der weitere Schwangerschaftsverlauf gestaltete sich unauffällig.

In 38+2 SSW wurde das Kind per sectionem geboren (Junge, $2770 \mathrm{~g}, 51 \mathrm{~cm}$, APGAR: 7/8/8, NA-pH 7,28).

Schlussfolgerung Die intrauterine Einlage eines Vorhofstents könnte eine therapeutische Möglichkeit bei restriktivem Foramen ovale mit Hydrops fetalis darstellen. Wir berichten von dem prä- und postnatalen Management.

\section{V3.005 Fallot'sche Tetralogie - Vergleich des Outcomes bei pränataler vs. postnataler Diagnose}

Autoren Wolter $A^{1}$, Enzensberger $C^{1}$, Kawecki $A^{1}$, Degenhardt $J^{2}$, Stressig $R^{2}$, Ritgen $J^{2}$, Thul $J^{3}$, Herrmann $J^{4}$, Axt-Fliedner $\mathrm{R}^{1}$

Institut 1 Zentrum für Frauenheilkunde und Geburtshilfe,

Universitätsklinikum Gießen \& Marburg, Gießen/DE; 2 praenatalplus.de, Köln/ DE; 3 Klinik für Kinderkardiologie und angeborene Herzfehler,

Universitätsklinikum Gießen \& Marburg, Gießen/DE; 4 Statistikberatung Gießen, Gießen/DE

DOI 10.1055/s-0037-1606887

Problemstellung Ziel unserer retrospektiven Auswertung war der OutcomeVergleich von Patienten mit pränatal diagnostizierter Fallot-Tetralogie versus Patienten mit postnataler Diagnosestellung sowie die Auswertung fetaler Klappendimensionen im Hinblick auf die Prädiktion einer frühen Intervention innerhalb 30 Tage postpartum.

Patienten und Methode Ausgewertet wurden 142 Patienten mit TOF, die zwischen 01/2008 und 06/2016 in unserem Kinderherzzentrum, unserer Pränataldiagnostik oder in der Praxis praenatal.de in Köln vorstellig waren. Bei 74 war die Diagnose pränatal bekannt, bei 68 wurde sie postpartal gestellt und an unser Herzzentrum überwiesen.

Ergebnis Unter den Patienten mit pränataler Diagnosestellung hatten 24/74 Feten $(32,4 \%)$ extrakardiale Fehlbildungen. 10/52 (19,2\%) wurden lebend geboren, 10 (19,2\%) waren postpartal lost for follow up. 12/42 (28,6\%) benötigten innerhalb von 30 Tagen eine Katheter- oder chirurgische Intervention. 1/42 (2,4\%) mit Trisomie 18 verstarb unmittelbar nach Geburt, 2/41 $(4,9 \%)$ nach Interventionen. Es handelte sich dabei um komplizierte Fälle mit Absent pulmonary valve Syndrom und Ductus arteriosus Agenesie.

In der Gruppe mit postnataler Diagnosestellung lag die Rate an extrakardialen Fehlbildungen bei 12/68 (17,6\%). Alle überlebten während des beobachteten Zeitraums, 7 (10,3\%) benötigten eine frühe Intervention.

Schlussfolgerung Es zeigten sich keine Unterschiede hinsichtlich post-interventionellem Überleben. Allerdings waren im Kollektiv mit pränataler Diagnosestellung mehr Patienten mit komplexen Fallot-Subtypen mit Pulmonalatresie und mehr extrakardiale Fehlbildungen als im Kollektiv mit postnataler Diagnosestellung. Im Kollektiv mit pränataler Diagnosestellung benötigten mehr eine frühe Intervention $(p=0,02)$. Es schienen nur die Dimensionen von linker und rechter Pulmonalarterie, nicht jedoch die von Aortenklappe und Truncus pulmonalis, gering prädiktiv für die Notwendigkeit einer Intervention innerhalb von 30 Tagen postpartum zu sein. 


\section{V3.006 Segmentale rechtsventrikuläre Displacement- und Gewebegeschwindigkeitsanalyse mittels color tissue Doppler imaging (c-TDI) bei Feten mit Hypoplastischem Linksherzsyndrom (HLHS)}

\author{
Autoren Graupner $\mathrm{O}^{1}$, Enzensberger $\mathrm{C}^{2}$, Wieg $\mathrm{L}^{1}$, Wolter $\mathrm{A}^{2}$, Khalil $\mathrm{M}^{3}$, \\ Yerebakan $\mathrm{C}^{3}$, Axt-Fliedner $\mathrm{R}^{2}$ \\ Institut 1 Klinikum rechts der Isar, Technische Universität München, \\ München/DE; 2 Universitätsklinikum Gießen und Marburg, Standort Gießen, \\ Gießen/DE; 3 Universitätsklinikum Gießen, Gießen/DE
}

DOI 10.1055/s-0037-1606888

Problemstellung Das Langzeit-Outcome von Feten mit HLHS (Hypoplastisches Linksherzsyndrom) wird durch die rechtsventrikuläre Funktion beeinflusst. Unsere Studie untersucht, ob und inwieweit sich Unterschiede in der segmentalen rechtsventrikulären Myokardauslenkung (Displacement) und in den segmentalen myokardialen Gewebegeschwindigkeiten von HLHS-Feten im Vergleich zu gesunden Feten nachweisen lassen.

Patienten und Methode Es handelt sich um eine prospektive Studie bestehend 20 Feten mit HLHS und 20 gesunden Kontrollfeten. Mithilfe der c-TDI (color tissue Doppler imaging) Technik wurden systolische und diastolische myokardiale Geschwindigkeiten sowie die Myokardauslenkung innerhalb verschiedener Segmente des rechten Ventrikels gemessen. Ein $3 \mathrm{~mm}$-ROI (region of interest) wurde hierzu im basalen, mittleren und apikalen Teil der rechten Ventrikelwand platziert. Mögliche Veränderungen der c-TDI-Parameter innerhalb der drei verschiedenen Segmente wurden in beiden Gruppen untersucht. Ergebnis HLHS-Feten zeigten im Vergleich zu den gesunden Kontrollefeten signifikant niedrigere e'-Geschwindigkeiten im Basal- und Mittelteil des rechten Ventrikels $(p<0,05)$. Basal gemessene Displacement-Werte waren bei HLHS-Feten niedriger als bei den gesunden Kontrollen. Bei den myokardialen Geschwindigkeiten und Displacement-Werten ergab sich eine signifikante Abnahme von der Basis bis zur Spitze des fetalen Herzens in beiden Gruppen (apikobasaler Gradient).

Schlussfolgerung Es konnte eine veränderte rechtsventrikuläre Myokardfunktion bei HLHS-Feten in unterschiedlichen Segmenten des rechten Ventrikels nachgewiesen werden. Ein apikobasaler Gradient zeigte sich sowohl in der Kontroll- als auch in der HLHS-Gruppe. Die c-TDI-Technik kann wertvolle Informationen über die fetale Myokardfunktion liefern. Ihre Bedeutung für das Monitoring sowie für die Vorhersage des Outcome betroffener HLHS-Feten muss jedoch noch endgültig geklärt werden.

\section{V3.007 Quantifizierung der mechanischen Dyssynchronie und Strainmessung mittels Speckle Tracking Echokardiografie bei Feten mit Wachstumsrestriktion und unauffälligen Kontrollen}

Autoren Krause $\mathrm{K}^{1}$, Möllers $\mathrm{M}^{1}$, Falkenberg $\mathrm{M}^{1}$, Klockenbusch $\mathrm{W}^{1}$, Schmitz R Institut 1 Klinik für Frauenheilkunde und Geburtshilfe, Münster/DE DOI 10.1055/s-0037-1606889

Problemstellung Evaluation der longitudinalen mechanischen Dyssynchronie bei unauffälligen Feten mittels „Specke Tracking“ Echokardiografie (STE) und Vergleich der longitudinalen mechanischen Dyssynchronie bei Feten mit Wachstumsrestriktion (FGR) und bei normalen Kontrollen.

Patienten und Methode In einer prospektiven Studie wurden 30 FGR und 62 unauffällige Feten, darunter 30 gestationsalter gepaarte Kontrollen, mittels STE im transversalem Vierkammerblick untersucht. Die Dyssynchronie wurde offline mittels QLab 9 (Philips Medical Systems, Andover, MA, USA) untersucht, als Zeitdifferenz der Umschlagpunkte des Strains in beiden Ventrikeln und dem Septum. Die inter- und intraventrikulären und die intraseptalen Dyssynchronien wurden erhoben und die inter- und die intraobserver Realibiltäten ausgewertet.

Ergebnis Die longitudinale mechanische Dyssynchronie war in allen Fällen mit hoher inter- und intraobserver Realibiltät darstellbar. Die Datenanalyse wurde mit einer hohen Bildfrequenz von durchschnittlich 175 Bildern/s durchgeführt. Der Grad der inter- und intraventrikulären Dyssynchronie war in der FGR Gruppe höher als in der normalen Kontrollgruppe.

Schlussfolgerung STE ist eine zuverlässige Technik zur Beurteilung der kardinalen Funktion des fetalen Herzens. Interventrikuläre Dyssynchronie könnte einen potentiellen Parameter zur frühen Detektion von subklinischer myokardialer Dysfunktion darstellen. Die zukünftige klinische Bedeutung der longitudinalen mechanischen Dyssychroniemessung sollte in größeren Studien und mit für die Pränataldiagnostik optimierter Technik weiterführend untersucht werden.

\section{V3.008 Vergleich des Nabelschnurarterien coiling index des Spender- und Empfänger-Zwillings beim feto-fetalen Transfusions-Syndrom vor fetoskopischer Lasertherapie}

Autoren Bamberg $\mathrm{C}^{1}$, Glosemeyer $\mathrm{P}^{1}$, Hecher $\mathrm{K}^{1}$

Institut 1 Universitätsklinikum Hamburg-Eppendorf, Hamburg/DE DOI 10.1055/s-0037-1606890

Problemstellung Nabelschnurarterien sind helixartig um die Nabelvene gewunden. Monochoriale Zwillinge mit einem feto-fetalen Transfusions-Syndrom haben eine einzigartige Kreislaufsituation: Der Empfänger-Zwilling weist eine Hyper- und der Spender-Zwilling eine Hypovolämie auf. Ziel der Arbeit ist der Vergleich des pränatalen Nabelarterien coiling index beim feto-fetalen Transfusions-Syndrom.

Patienten und Methode In der Abteilung für Geburtshilfe und Pränatalmedizin, Universitätsklinikum Hamburg-Eppendorf haben wir bei $\mathrm{n}=28$ Schwangeren 3-D-Volumina der Nabelschnur mit Farbdopplersonografie vom Empfänger- und Spender-Zwilling mit einem feto-fetalen Transfusions-Syndrom unmittelbar vor einer Lasertherapie akquiriert. Die Analyse erfolgte retrospektiv. Im Multiplanarmodus wurde die Nabelschnur horizontal dargestellt und vergrößert sowie der coling index (Kehrwert der Distanz $(\mathrm{cm})$ einer gesamten Arterienumwindung) gemessen. Ein coiling index zwischen 0,2 und 0,6 wurde als normal klassifiziert. Hyper- und hypocoiled Nabelarterien lagen vor, wenn die 90 . Perzentile $(0,6)$ über- bzw. die 10 . Perzentile $(0,2)$ unterschritten wurde.

Ergebnis Das mittlere ( \pm STD) Gestationsalter betrug $21 \pm 2,4$ SSW. 51 Volumina der Nabelschnur wurden ausgewertet. Der coiling index konnte bei 27 von 28 Spender- und 24 von 28 Empfänger-Feten gemessen werden. Es fand sich ein signifikanter Unterschied $(p=0,01)$ bezüglich des coiling index in beiden Zwillingspopulationen. Empfänger-Zwillinge hatten in 14 (52\%) Fällen einen normalen coiling index. Zwei Feten (7,5\%) zeigten eine hypo- und elf $(40,5 \%)$ wiesen eine hypercoiled Nabelarterie auf. Spender-Zwillinge hatten in zehn $(41,5 \%)$ Fällen ein normales coiling. Bei zehn $(41,5 \%)$ Feten wurde ein hypocoiling und bei vier (17\%) Feten ein hypercoiling nachgewiesen.

Schlussfolgerung Spender- und Empfänger-Zwillinge zeigten signifikant unterschiedliche Muster des Nabelschnur coiling. Dies kann als Folge der unterschiedlichen Kreislaufsituationen beim feto-fetalen Transfusions-Syndrom interpretiert werden.

\section{V3.009 Einfluss von feto-fetalem Transfusionssyndrom auf den Tei-Index von Donor und Rezipient}

Autoren Ortiz J $]^{1}$, Torres $\mathrm{X}^{2}$, Eixarch $\mathrm{E}^{2}$, Lobmaier $\mathrm{S}^{1}$, Martinez $]^{2}$, Gratacós $\mathrm{E}^{2}$, Crispi $\mathrm{F}^{2}$

Institut 1 Klinikum rechts der Isar, München/DE; 2 BCNatal, Barcelona/ES DOI 10.1055/s-0037-1606891

Problemstellung Auswertung der Zeitintervalle und des Tei-Index bei Zwillingen mit feto-fetalem Transfusionssyndrom (FFTS).

Patienten und Methode Linksventrikuläre isovolumetrische Relaxations- (IRT) und Kontraktionszeit (ICT) sowie Ejektionszeit (ET) wurden mittels gepulster Doppler-Sonografie bei 51 Zwillingspaaren mit feto-fetalem Transfusionsyn- 
drom innerhalb von $24 \mathrm{~h}$ vor und $72 \mathrm{~h}$ nach der Lasertherapie gemessen. 47 Zwillingspaare unauffälliger monochorialer Geminigraviditäten wurden ebenfalls analysiert und als Kontrollgruppe eingeschlossen.

Ergebnis Die Donorfeten wiesen eine kürzere ET als die Rezipienten und Kontrollzwillinge sowie einen höheren Tei-Index als die Kontrollzwillinge auf. Außerdem zeigten die Rezipienten längere IRT, längere ICT und höheren TeiIndex als die Donorfeten und Kontrollzwillinge. Prä-interventionell war der TeiIndex in allen FFTS-Stadien erhöht. Post-interventionell verbesserten sich die ET bei den Donorfeten in allen FFTS-Stadien und die IRT bei den Rezipienten in Frühstadien. (Alle o.g. Ergebnisse waren statistisch signifikant).

Schlussfolgerung Die myokardiale Funktion war sowohl bei den Donorfeten als auch bei den Rezipienten sogar in Frühstadien eingeschränkt. Während die Donorfeten eine systolische Funktionsstörung wahrscheinlich aufgrund einer Hypovolämie aufwiesen, zeigten die Rezipienten eine globale Funktionsstörung am ehesten wohl wegen einer Hypervolämie und erhöhter Nachlast. Die Lasertherapie konnte die myokardiale Funktion schon innerhalb von $72 \mathrm{~h}$ beeinflussen.

\section{V3.010 Höhergradige Mehrlingsschwangerschaften - Management und Ausgang}

Autoren Geiler $\mathrm{S}^{1}$, Riße $\mathrm{S}^{1}$, Stepan $\mathrm{H}^{2}$, Faber $\mathrm{R}^{1}$

Institut 1 Zentrum für Pränatale Medizin, Leipzig/DE; 2 Universitätsklinik Leipzig, Leipzig/DE

DOI 10.1055/s-0037-1606892

Problemstellung Höhergradige Mehrlingsschwangerschaften (hMS > 2 Feten) gehen mit erhöhter perinataler Morbidität und Mortalität einher. Inwieweit das Ziel einer langen ambulanten Betreuung gerechtfertigt ist, soll die Auswertung der am Zentrum für Pränatale Medizin Leipzig (ZPML) betreuten Schwangerschaften zeigen.

Patienten und Methode Im Zeitraum von 2007 - 2017 wurden insgesamt 51 höhergradige Mehrlingsschwangerschaften betreut, deren perinataler Ausgang bekannt ist. In einer prospektiven Beobachtungsstudie vergleichen wir Verlauf und Ausgang ohne und mit Fetenreduktion (FR). Dabei wird besonders auf die Länge der ambulanten Betreuung sowie auf das Gestationsalter bei Geburt geachtet.

Ergebnis Von den 51 Schwangerschaften $(44 \times$ Drillinge, $6 \times$ Vierlinge, $1 \times$ Fünflinge) wurden 28 Schwangerschaften ohne FR betreut (27 Drillingsschwangerschaften und eine monochoriale Vierlings-SS bis zur 28+0 SSW). Gegenübergestellt wurden Drillings-SS mit Fetenreduktion auf Zwillinge $(n=12)$ und exspektativ betreute Drillingsschwangerschaften $(n=27)$. In der exspektativen Gruppe betrug das mittlere SS-alter bei Geburt 32+1 SSW, das mittlere Geburtsgewicht 1653 Gramm. Die stationäre Aufnahme erfolgte durchschnittlich in der 30+1 SSW. Es kam bei 4 Feten zum IUFT. In der Gruppe mit FR betrug das mittlere SS-alter bei Geburt 34+4 SSW, das Geburtsgewicht 2017 Gramm. Die Hospitalisierung erfolgte durchschnittlich in der 33+6 SSW. Es traten 3 IUFTs auf.

Schlussfolgerung Eine exspektative Betreuung bei Drillingsschwangerschaften ist durchaus gerechtfertigt; sie geht mit einer leicht erhöhten Morbidität einher. Demgegenüber stehen psychische Belastung einer Reduktion sowie das Eingriffskomplikationsrisiko. Frühgeburtssymptome (vorzeitiger Blasensprung, vorzeitige Wehentätigkeit, Cervixinsuffizienz) sind die entscheidenden Indikationen für eine stationäre Überwachung. Die Ergebnisse des Ausgangs der Drillingsschwangerschaften sind vergleichbar mit denen der Literatur und rechtfertigen somit den Ansatz für eine lange ambulante Betreuung in einem Pränatalzentrum.

\section{5: Gyn/Pränatal II}

\section{V5.001 Hypoplasie des Vermis cerebelli bei Feten mit 45 bis $84 \mathrm{~mm} \mathrm{SSL}$}

Autoren Altmann $\mathrm{R}^{1}$, Specht $\mathrm{C}^{2}$, Hechenberger $\mathrm{C}^{2}$, Scharnreitner I ${ }^{1}$, Arzt $\mathrm{W}^{1}$, Scheier $\mathrm{M}^{2}$

Institut 1 Kepleruniversitätsklinik Linz, Linz/AT; 2 Institut für Fetalmedizin, Feldkirch/AT DOI 10.1055/s-0037-1606893

Problemstellung Beschreibung der Hypoplasie/Aplasie des Vermis cerebelli bei Feten mit 45 - 84 mm Scheitelsteißlänge.

Patienten und Methode Wir zeigen die Ergebnisse einer prospektive Beobachtungsstudie, in die 66 Feten inkludiert wurden, deren Mütter unsere Zentren für ein Ersttrimesterscreening bzw. einen Combined Test aufsuchten. Die inkludierten Feten zeigten entweder eine Strukturanomalie oder einen auffälligen Kariotyp. Bei diesen Feten wurden transvaginal 3D Volumenblöcke der Fossa posterior aufgenommen und archiviert. Die Ergebnisse der Messungen der Fossa posterior dieser Feten wurden korreliert mit dem Normalkollektiv von 216 Feten mit normaler Entwicklung.

Ergebnis In 6 der 66 Feten (11\%) war der Vermis unter der 5. Perzentile des gestationsalter korrelierten Normwerts. Bei 4 Feten wurde die Schwangerschaft wegen Trisomie 13/18 bzw. Holoprosencephalie beendet. Eine Schwangerschaft zeigte im Ultraschall der 20. SSW eine Dandy Walker Malformation, eine weitere eine Vermishypoplasie.

Schlussfolgerung Eine Hypoplasie/Aplasie des Vermis cerebelli kann im transvaginalen 3D Ultraschall ab der 12 . Schwangeachaftswoche erkannt werden.

\section{V5.002 Differentialdiagnose intrazerebrale Raumforderung im späten 2. Trimenon: Solitärer fibröser Tumor vom Hämangioperizytom-Typ}

Autoren Mommsen $\mathrm{H}^{1}$, Minderer $\mathrm{S}^{1}$, Müller $\mathrm{AM}^{2}$, Schramm $\mathrm{T}^{1}$ Institut 1 Pränatal-Medizin München, München/DE; 2 Zentrum für Kinderpathologie und Pathologie, Bonn/DE DOI 10.1055/s-0037-1606894

Problemstellung Intrazerebrale Raumforderungen treten oft erst in der zweiten Schwangerschaftshälfte auf. Häufig handelt es sich um große Einblutungen (fetal stroke). Selten kann es sich auch um einen Tumor handeln. Die Differentialdiagnose ist entscheidend für die Prognoseeinschätzung.

Patienten und Methode 31 J, IIG/IP, Erstvorstellung 24+5. SSW. Z.n. unauffälligem Screening Ilb in der 21. SSW:

$57 \times 42 \mathrm{~mm}$ große inhomogene gering perfundierte Raumforderung in der linken Hemisphäre/Ventrikel bis zum Hirnstamm/Kleinhirn reichend, Mittellinie verlagert. Hohe Vmax und reverse flow in der ACM (Anämie DD Hirndruck). KU 95. Perz. V.a. Tumor DD Massenblutung.

25+3: Befund progredient $70 \times 57 \mathrm{~mm}$, Hirndruckzeichen zunehmend, verschmälerter Cortex, Ventrikulomegalie, KU > 99. Perz. FBS: normales Karyogramm; moderate Anämie Hb 8,3 g/dl. Dringender V.a. Tumor.

25+5: fetales MRT: Riesiger verdrängender Tumor der linken Hemisphäre, z.B. Teratom oder Plexustumor. Prognose infaust.

26+2: ausführliche interdisziplinäre Beratung nach erneuter Sonografie (weiter progredient); V.a. anaplastischer Tumor. Paar entscheidet sich nach Bedenkzeit für späten Abbruch (nach Fetozid).

27+1: Fetozid; Befund weiter progedient, kein normales Gehirngewebe mehr, extremes Schädelwachstum. Kardiale Belastung sichtbar. 


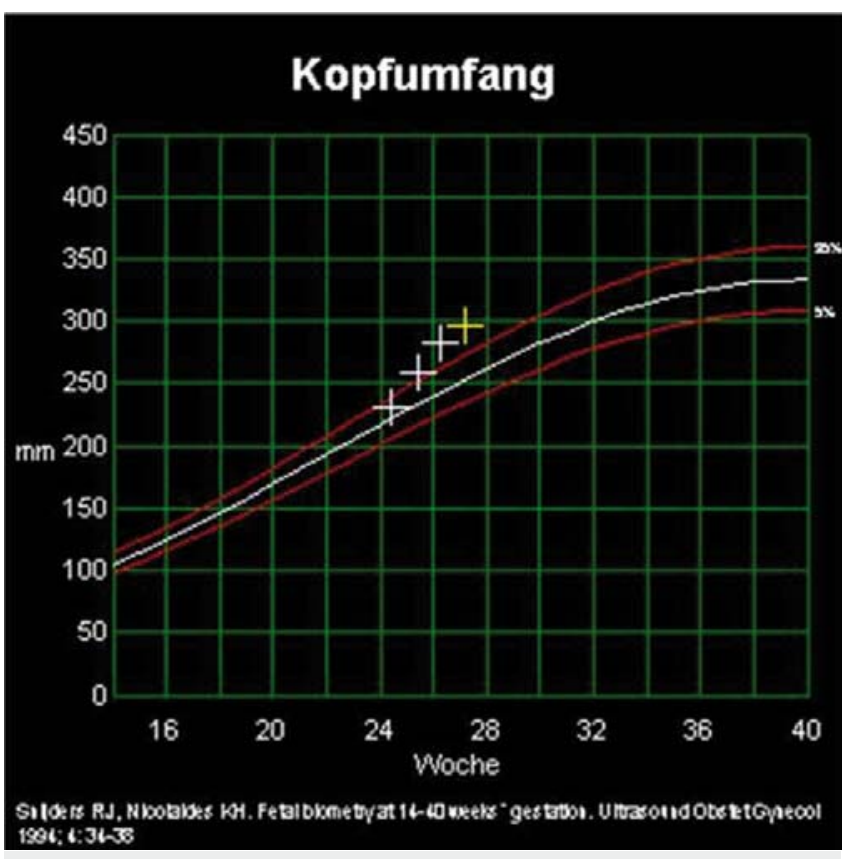

Abb. 1 Gehirntumor: Kopfwachstum

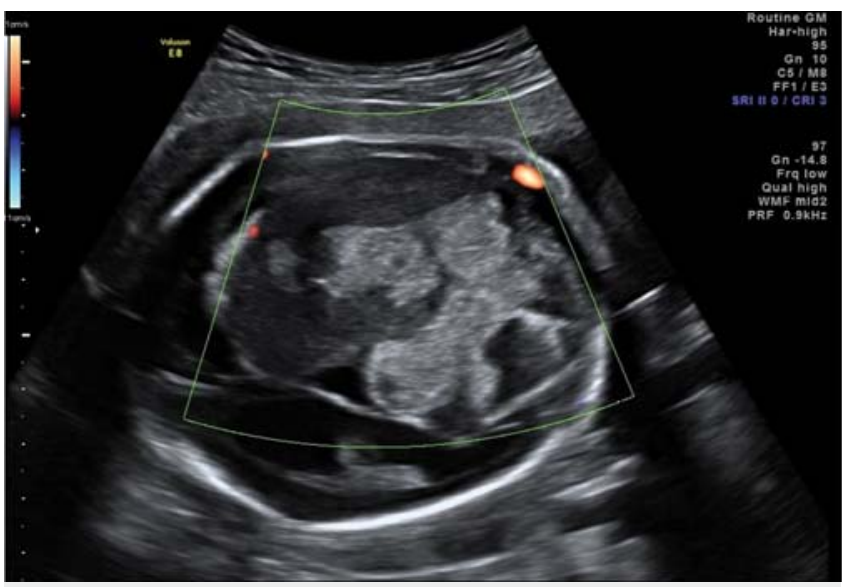

Abb. 2 Intrazerebrale Raumforderung linke Hemisphäre

Ergebnis Totgeburt 27+2. SSW nach Misoprostol-Einleitung: Weiblich, $1170 \mathrm{~g}$, SSL $23 \mathrm{~cm}, \mathrm{KU} 29 \mathrm{~cm}$ (>99. Perz).

Histologie/Immunhistochemie: $8 \mathrm{~cm}$ großer gefäßreicher spindelzellliger Hirntumor der linken Hemisphäre mit zahlreichen Einblutungen und Nekrosen. Rasches Wachstum durch zahlreiche Einblutungen mitverursacht.

Diagnose: Hämangioperizytom.

Schlussfolgerung Auch nach dem 2. Screening können sich schwere Entwicklungsstörungen insbesondere des Gehirnes zeigen. Als zusätzliches bildgebendes Verfahren kann die fetale MRT ein wichtige differentialdignostische Ergänzung sein.

Ein intrazerbraler SFT vom Hämangioperizytomtyp ist eine sehr seltene hochmaligne Entität, die zusammen mit intratumoralen Einblutungen die rasante Befundprogredienz erklärt. Bei infauster Prognose muss ein später Abbruch der Schwangerschaft diskutiert werden.

\section{V5.003 Outcome von 1169 Feten mit pränatal diagnostizierter singulärer Nabelschnurarterie (SUA)}

Autoren Friebe-Hoffmann $\mathrm{U}^{1}$, Hiltmann $\mathrm{A}^{1}$, Friedl $\mathrm{T}^{1}$, Lato $\mathrm{K}^{1}$, Hammer $\mathrm{R}^{2}$, Janni $W^{1}$, Kozlowski $P^{2}$

Institut 1 Universität Ulm, Ulm/DE; 2 praenatal.de, Düsseldorf/DE

DOI 10.1055/s-0037-1606895

Problemstellung Die pränatal diagnostizierte singuläre Nabelschnurarterie (SUA) hat eine Inzidenz von 0,5\% und ist oft mit einem adversen fetalen Outcome vergesellschaftet. Aufgrund inkohärenter Literatur wird das Management der SUA-Feten immer wieder kontrovers diskutiert.

Patienten und Methode 1169 Feten mit pränatal diagnostiziertem SUA im Zeitraum von 1997 - 2014 wurden einer beschreibenden retrospektiven Single-Center-Analyse hinsichtlich Fehlbildungsraten, Schwangerschaftsverlauf und perinatalem Outcome unterzogen.

Ergebnis Bei 989 (84,6\%) der Feten lag eine isolierte SUA (iSUA) vor, während $180(15,4 \%)$ zusätzliche strukturelle und/oder chromosomale Auffälligkeiten zeigten. Diese verteilten sich wie folgt: 9,0\% kardiovaskulär, 3,5\% urogenital, 2,9\% muskuloskeletal, 3,0\% gastrointestinal, 2,1\% zerebral und 2,8\% chromosomal. 50,8\% (49,2\%) der Feten waren weiblich (männlich) und in 64,2\% vs. $35,8 \%$ lag eine rechte vs. linke SUA vor. Feten mit zusätzlichen Anomalien wiesen insgesamt eine niedrigere Rate an Lebendgeburten $(85,0 \%$ vs. $98,5 \%, \quad p<0,001)$, ein niedrigeres mittleres Geburtsgewicht (2825 vs. $3220 \mathrm{~g}, \mathrm{p}<0,001),(13,7 \%$ vs. 3,8\%, $\mathrm{p}<0,001)$ und eine höhere rate an small for gestational age (SGA) Babys (21,6\% vs. 9,3\%, p<0,001) auf. In 5,1\% (60) der Kinder wurden chromosomale oder strukturelle Anomalien erst post partum nachgewiesen.

Schlussfolgerung Die Diagnose der fetalen SUA sollte ein intensives Organscreening nach sich ziehen und bei assoziierten Fehlbildungen eine invasive Diagnostik angeboten werden. Bei Feten mit iSUA werden intermittierende Biometriekontrollen zur Früherkennung einer intrauterinen Wachstumsbeschränkung (IUGR) empfohlen.

\section{V5.004 Prognosekriterien bei fetalen Steißbeinteratomen}

Autoren Geiler $\mathrm{S}^{1}$, Riße $\mathrm{S}^{1}$, Stepan $\mathrm{H}^{2}$, Faber $\mathrm{R}^{1}$

Institut 1 Zentrum für Pränatale Medizin, Leipzig/DE; 2 Universitätsklinik

Leipzig, Leipzig/DE

DOI 10.1055/s-0037-1606896

Problemstellung Fetale Steißbeinteratome können bereits intrauterin eine vitale Bedrohung des Feten darstellen. Fragestellung unserer Analyse war, ob bereits bei Erstdiagnose eindeutige Prognosekriterien für die weitere fetale Entwicklung vorliegen.

Patienten und Methode Im Zeitraum von 2007 - 2016 wurden im Zentrum für Pränatale Medizin Leipzig (ZPML) alle Schwangerschaften einbezogen, bei denen die Diagnose eines Steißbeinteratoms $(S C T=13)$ gestellt wurde. Es erfolgte eine retrospektive Datenanalyse unter Einbeziehung bestimmter Prognosekriterien.

Ergebnis Von den 13 Feten mit Steißbeinteratom wurden 5 Schwangerschaften ausgetragen (lebt $n=5$ ), 5 Frauen entschieden sich für eine Terminierung (TOP $n=5), 2$ Feten verstarben intrauterin (IUFT $n=2$ ), ein Kind verstarb unmittelbar postnatal an den Folgen der Tumorerkrankung. Bei einem der 5 lebend geborenen Kinder wurde die Diagnose erst postnatal gestellt. Bei den anderen 4 Kindern war bei pränataler Erstdiagnose die tumor volume to fetal weight ratio TFR gering $(<0,12)$ die ACM wies eine normale Geschwindigkeit auf und das Flussprofil im Ductus venosus war normal. Bei den 5 Feten, bei denen sich die Schwangere für ein TOP entschieden hatte, zeigten 3 Feten bei Erstvorstellung bereits Anämie, Perikarderguss, Kardiomegalie oder Hautödem. Die TFR war jeweils größer als 0,12. Auch bei den 2 intrauterin verstorbenen Feten war die TFR größer als 0,12. Bei weiteren zwei Feten mit zwar geringer TFR wurde aus mütterlicher Indikation bzw. bei intraduraler Mitbeteiligung des Tumors eine Termination durchgeführt. 
Schlussfolgerung Die Tumorgröße in Abhängigkeit vom Fetalgewicht (TFR) scheint bereits bei pränataler Erstdiagnose entscheidend für die Prognoseeinschätzung zu sein. Die sonografische Kontrolle des Tumorwachstums, der Arteria cerebri media und des Ductus venosus sowie der Fruchtwasserverhältnisse bestimmen Management und Entbindungszeitpunkt.

\section{V5.005 Prognostische Bedeutung der Ultraschallzeichen bei Arthrogryposis multiplex congenita}

Autoren Busack $B^{1}$, Henrich $W^{2}$, Verlohren $S^{3}$

Institut 1 Charité Universitätsmedizin Berlin, Berlin/DE; 2 Charité -

Universitätsmedizin Berlin, Berlin/DE; 3 Charite-Universitätsmedizin Berlin, Berlin/DE

DOI 10.1055/s-0037-1606897

Problemstellung Die fetale Arthrogryposis multiplex congenita (AMC) ist ein heterogener Symptomkomplex, der durch das Auftreten von Gelenkfehlstellungen in mindestens zwei Körperregionen definiert ist. Das Spektrum der möglichen Auffälligkeiten im Ultraschall ist vielfältig und die prognostische Bedeutung unklar, was die Beratungssituation verkompliziert.

Patienten und Methode In dieser monozentrischen, retrospektiven Studie wurde die prognostische Bedeutung der Ultraschallauffälligkeiten bei V.a. AMC untersucht. Eingeschlossen wurden 41 Schwangerschaften, die sich zwischen 1999 und 2016 in der Pränataldiagnostik der Charité - Universitätsmedizin Berlin vorstellten.

Ergebnis Die Ultraschallzeichen betrafen Sprungelenk/Fuß $(63,4 \%)$, Knie (53,6\%), Ellenbogen (60,9\%), Handgelenk (48,7\%), sowie Fingerfehlstellungen (36,5\%). In 36,5\% der Fälle kam es zu einem Hydrops fetalis. in 24,3\% konnte keine Magenfüllung dokumentiert werden. Profilauffälligkeiten wurden bei $26,8 \%$ diagnostiziert.

Daten zum Schwangerschaftsausgang lagen bei 34/41 Schwangerschaften vor: in 14 Fällen wurde eine Schwangerschaftsbeendigung vorgenommen, zweimal kam es zum IUFT. 18 Kinder wurden lebend geboren, 6 verstarben unmittelbar postpartal. Ein Hydrops fetalis zeigte sich bei $50 \%$ der Feten, die nach Geburt verstarben, bei 52,9\% Beendigungen gegenüber 0\% der überlebenden Feten. Kinder, die unmittelbar postpartal verstarben hatten in 23,5\% der Fälle eine Skoliose und 5,8\% ein Ophistotonus gegenüber 0\% in der Gruppe der Überlebenden. Eine fehlende Magenfüllung war bei $66,6 \%$ der postpartal verstorbenen, $29,4 \%$ der präpartal verstorbenen sowie $8,3 \%$ der überlebenden Kinder zu sehen.

Schlussfolgerung Zusätzlich zu den definierenden Ultraschallzeichen konnten Ultraschallzeichen mit prognostischer Bedeutung identifiziert werden. Insbesondere der Hydrops fetalis, die Skoliose sowie fehlenden Magenfüllung waren mit ungünstigem Ausgang vergesellschaftet. Dieses Wissen kann zukünftig bei Beratungen helfen, die Prognose besser abzuschätzen.

\section{V5.006 Vesico-amnialer Shunt mit dem Somatex-Shuntsystem bei fetaler Megazystis}

\author{
Autoren Strizek B ${ }^{1}$, Berg $C^{1}$, Gembruch $U^{1}$, Geipel $A^{1}$ \\ Institut 1 Universitätsfrauenklinik Bonn, Bonn/DE \\ DOI 10.1055/s-0037-1606898
}

Problemstellung Vesico-amniale Shuntanlagen (VAS) bei fetaler Megazystis haben hohe Komplikationsraten. Beim neuartigen Somatex-Shuntsystem sollen kleine Schirme an den Enden eine Dislokation verhindern, zudem ist aufgrund der kleineren Punktionskanüle eine VAS bereits am Ende des ersten Trimenons möglich.

Patienten und Methode Retrospektive, unizentrische Studie aller seit 11/ 2014 mit dem intrauterinen Somatex-Shuntsystem behandelten Patientinnen hinsichtlich Dislokationsrate, Komplikationen und postnatalem pulmonalem Outcome.

Ergebnis Bei 23 Patientinnen erfolgte eine VAS mit dem Somatex-Shuntsystem mit durchschnittlich $16,8 \mathrm{SSW}(14,4-28,0 \mathrm{SSW})$. Es erfolgten $1-3$
Shuntanlagen pro Schwangerschaft. Bei 10 Feten kam es nach initial guter Lage des Shunts nach durchschnittlich 59,6 Tagen (1 - 128 d) zu einer Dislokation. In der Mehrzahl der Fälle erfolgte diese nach intraabdominal, so dass in 6 Fällen eine weitere abdomino-amniale Shuntanlage erfolgte. In einem Fall kam es außerdem zu einer Migration des Shunts durch die Uteruswand in das maternale Abdomen.

Das Alter bei Geburt lag bei durchschnittlich 35,5 SW (28,7 - 40,4). Es kam zu einem Abort und einem Abbruch der Schwangerschaft bei fetaler Niereninsuffizienz in der 31. SSW.

Von derzeit 17 geborenen Kinder überlebten 15 die Neonatalperiode. Ein Kind verstarb an einer Lungenhypoplasie, ein weiteres bei gleichzeitiger Zwerchfellhernie. In mehr als $50 \%$ der Fälle musste postnatal ein operativer Eingriff zur Entfernung des Shunts durchgeführt werden.

Schlussfolgerung Das Somatex-Shuntsystem zur vesiko-amnialen Shuntanlage zeigt eine im Vergleich zu anderen Shunts geringere Dislokationsrate und langes Intervall bis zur Dislokation. Letale Lungenhypoplasien scheinen im Vergleich zu anderen Shuntsystemen verringert, ob Auswirkungen auf die Nierenfunktion bestehen, bleibt aktuell Gegenstand weiterer Untersuchungen.

\section{V5.007 Thorako-amniale Shuntanlage bei fetalem Hydrothorax: erste Erfahrungen mit dem Somatex intrauterinen Shuntsystem}

Autoren Strizek B ${ }^{1}$, Berg $C^{1}$, Gembruch $U^{1}$, Geipel $A^{1}$

Institut 1 Universitätsfrauenklinik Bonn, Bonn/DE

DOI 10.1055/s-0037-1606899

Problemstellung Thorako-amniale Shuntanlagen (TAS) bei fetalem Hydrothorax sind mit hohen Dislokationsraten verbunden. Wir berichten über erste Erfahrungen mit dem intrauterinen Somatex-Shuntsystem, das aufgrund seiner Materialeigenschaften diese verhindern soll.

Patienten und Methode Retrospektive, unizentrische Analyse aller seit 10/ 2014 durchgeführten thorako-amnialen Shuntanlagen in unserem Perinatalzentrum hinsichtlich technischer Aspekte, Dislokationrate und Outcome.

Ergebnis Im Untersuchungszeitraum wurden 49 TAS bei 25 Patientinnen mit durchschnittlich 27 SSW (19-23 SSW) durchgeführt. Die Indikationen waren Hydrothorax und CPAM mit Hydrops fetalis.

Bei 18 Patientinnen wurde initial ein Somatex-Shunt verwendet, in 4 Fällen (22\%) war eine Drainage durch die initiale Shuntanlage nicht suffizient. In 4 weiteren Fällen trat im Verlauf eine Dislokation auf. In 7 Fällen wurden erneute TAS (1 - 3/Patientin) durchgeführt, damit konnte bei 11/18 Fällen eine komplette Drainage und in den weiteren Fällen eine klinisch suffiziente Drainage erreicht werden. Das durchschnittliche Gestationsalter bei Geburt betrug 35,2 SSW (28 - 39).

Es trat ein intrauteriner Fruchttod und ein neonataler Tod bei Lungenhypoplasie auf, 13 Kinder wurden lebend entlassen. 3 Kinder waren lost to follow-up.

Bei 5 Kindern war postnatal eine operative Entfernung des Somatex-Shunts notwendig und bei einer Mutter war der Shunt in die Uteruswand migriert, der im Rahmen der Sectio komplikationslos entfernt wurde.

Schlussfolgerung In ca. $40 \%$ der Fälle wird nach initialem Einsatz des Somatex-Shuntsystems bei TAS eine Re-Intervention notwenig. Allerdings ist auch nach wiederholten Punktionen in der Mehrheit der Fälle eine komplette Drainage mit gutem Outcome zu erreichen. In einigen Fällen muss der Shunt nach Migration des Systems nach intrathrorakal operativ entfernt werden. 


\section{V5.008 Fetale Ovarialzysten - Pränatale Diagnose, prä- und postnataler Verlauf}

\author{
Autoren Takacs $\mathrm{FZ}^{1}$, Hamza $\mathrm{A}^{1}$, Meier $\mathrm{CM}^{2}$, Zemlin $\mathrm{M}^{2}$, Meyberg- \\ Solomayer $\mathrm{GC}^{1}$ \\ Institut 1 Universität des Saarlandes, Homburg/DE; 2 Universität des \\ Saarlandes, homburg/DE \\ DOI 10.1055/s-0037-1606900
}

Problemstellung In der vorliegenden Arbeit wurde untersucht, ob ein Zusammenhang zwischen den sonografischen Parametern bei fetalen Ovarialzysten und dem postnatalen Verlauf besteht, vor allem im Hinblick auf die Notwendigkeit einer postnatalen chirurgischen Intervention und einem Verlust des Ovars.

Patienten und Methode Zu der vorliegenden retrospektiven Studie wurden 41 Fälle mit fetalen Ovarialzysten untersucht. Postnatal war der Verlauf bei 28 Kindern bekannt.

Ergebnis Bei 16 Fällen (57\%) fand sich eine Spontanauflösung. 7 (44\%) pränatal und $9(56 \%)$ postnatal. Es handelte sich dabei um einfache $(n=15 ; 94 \%)$, $<5 \mathrm{~cm}(\mathrm{n}=14 ; 88 \%)$ bzw. kleiner als $4 \mathrm{~cm}(\mathrm{n}=11 ; 69 \%)$ Zysten. Jeweils die Hälfte war rechts bzw. linksseitig. Eine Operation wurde bei 12 von 28 (43\%) der Kinder durchgeführt. Die Indikationen waren Raumforderung, Stieldrehung oder Ruptur. Bei einer, bereits bei der Erstvorstellung rupturierten Zyste konnte kein valider Durchmesser gemessen werden. Die operierten Zysten waren überwiegend rechtsseitig $(n=9 ; 75 \%)$, komplex $(n=7 ; 58 \%)$, und $\geq 4 \mathrm{~cm}(11 / 11,100 \%)$ bzw. $\geq 5 \mathrm{~cm}(8 / 11 ; 73 \%) .5$ (42\%) der 12 operierten Kinder hatten eine Oophorektomie. Eine Zyste von $73 \mathrm{~mm}$ wurde in der 32 SSW punktiert und entleert. Im Verlauf kam es zu einer Einblutung. Postnatal wurde eine Zystenentfernung durchgeführt.

Schlussfolgerung Das Risiko für Komplikationen bzw. dem Verlust des betroffenen Ovars, ist größer bei rechtsseitigen, komplexen, großen $(\geq 4 \mathrm{~cm})$ fetalen Ovarialzysten. Um Komplikationen rechtzeitig zu entdecken sind postnatale Kontrolluntersuchungen notwendig.

\section{V5.009 Thorax-zu-Kopf-Quotient und Defektdurchmesser-zu-Kopf-Quotient als Prädiktoren für das fetale Outcome bei großen Omphalocelen}

Autoren Braun $\mathrm{J}^{1}$, Diemon $\mathrm{N}^{1}$, Funke $\mathrm{K}^{1}$, Möllers $\mathrm{M}^{1}$, Hammer $\mathrm{K}^{1}$, Steinhard $\mathrm{J}^{2}$, Sauerland $C^{1}$, Müller $\mathrm{V}^{1}$, Klockenbusch $\mathrm{W}^{1}$, Schmitz $\mathrm{R}^{3}$

Institut 1 Universitätsklinik Münster, Münster/DE; 2 Universitätsklinikum der Ruhr-Universität Bochum, Bad Oeynhausen/DE; 3 Klinik für Frauenheilkunde und Geburtshilfe, Münster/DE

DOI 10.1055/s-0037-1606901

Problemstellung Ziel der Arbeit war es das Verhältnis des Thoraxdurchmessers zum Defektdurchmesser von großen Omphalocelen als Vorhersagewert für das fetale Outcome zu untersuchen.

Patienten und Methode In dieser retrospektiven Arbeit wurden 17 Feten mit isolierter großer Omphalocele untersucht. Der anteriore-posteriore Thoraxdurchmesser und der Defektdurchmesser wurden sonografisch vermessen. Hierbei wurden der Thorax-zu-Kopf-Quotient (T/HC), der Defektdurchmesser-zu-Kopf-Quotient (DD/HC) und der Quotient aus Defektdurchmesser und Thoraxdurchmesser $(\mathrm{DD} / \mathrm{T})$ berechnet. Die Dauer der Beatmung postpartal $\left(\mathrm{t}_{\text {ventilation }}\right)$, die Dauer des Krankenhausaufenthaltes ( $\left.\mathrm{t}_{\text {hospital }}\right)$ und die Art der Behandlung postpartal wurde als Outcome-Parameter angegeben.

Ergebnis Es wurde weder hinsichtlich der Krankenhausaufenthaltsdauer $t_{\text {hospital }}(r=-0,418, p=0,095 ; r=-0,153, p=0,556 ; r=-0,023, p=0,929)$ noch der Beatmungsdauer $t_{\text {ventilation }}(r=-0,391, p=0,121 ; r=0,041, p=0,875$; $r=0,121, p=0,645)$ ein Zusammenhang mit den berechneten Quotienten gefunden ( $T / H C, D D / H C$ oder $D D / T$ ). Auch die Art der postnatalen Behandlung war weder mit einem der drei berechneten Quotienten noch der Krankenhausaufenthaltsdauer $t_{\text {hospital }}$ assoziiert $(r=0,155, p=0,553 ; r=0,019$, $p=0,942$; und $r=0,012, p=0,965 ; r=-0,009, p=0,973)$. In $53 \%$ der Fälle

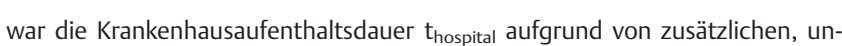
abhängigen postnatalen Komplikationen verlängert.

Schlussfolgerung Der Thoraxdurchmesser und der Defektdurchmesser groBer Omphalocelen können nicht als Prädiktor für das fetale Outcome herangezogen werden. Die perinatale Betreuung dieser Fälle stellt eine interdisziplinäre Herausforderung dar. Durch die Betreuung in einem Perinatalzentrum wird das fetale Outcome begünstigt.

\section{V5.010 Ultrasound markers in fetal hydronephrosis to predict postnatal surgery}

Authors Wertaschnigg $D^{1}$, Wohlmuth $C^{1}$, Schimke $C^{2}$, Kiener TA ${ }^{1}$ Institute 1 Universitätsklinik, Salzburg/AT; 2 Universitätskinderklinik, Salzburg/AT

DOI 10.1055/s-0037-1606902

Purpose Parents confronted with the finding of antenatal hydronephrosis are particularly interested in whether their baby will need postnatal surgery. The objective of this study was to predict neonatal nephrouropathy requiring surgery on the basis of the fetal anteroposterior renal pelvic diameter and the Society for Fetal Urology SFU grading system.

Methods The medical records of 179 patients with the finding of antenatal hydronephrosis were reviewed retrospectively. Additionally ANH was graded according to the SFU grading system. Prenatal ultrasound examinations were correlated to postnatal outcome, which was divided into three groups: prenatal resolution, conservative management and surgical treatment.

Results 58 (32,4\%) cases were classified as prenatal resolution, 89 (49,7\%) babies were assigned to the conservative outcome group and 32 (17,9\%) patients needed surgical repair. Postnatal surgery was best predicted in the second trimester (area under the receiver operating characteristics curve: 0,839 ) by an anteroposterior renal pelvic diameter cut-off of $8,25 \mathrm{~mm}$ (sensitivity: $77,8 \%$; specificity: $85,7 \%$; PPV of $53,9 \%$, NPV of $94,7 \%$ ). The combination of the parameters progression of SFU grade and SFU grade 3 or 4 achieved a sensitivity of $84,4 \%$ and a specificity of $80,3 \%$ for the prediction of a surgical outcome.

Conclusion Second trimester ultrasound is suitable for the prediction of neonatal nephrouropathy requiring surgery. The SFU grade should be assessed in every prenatal ultrasound examination as some further risk estimates can be made based on its dynamics over time.

\section{V5.011 Pränatale Diagnose einer kongenitalen neuromuskulären Erkrankung}

Autoren Scharnreitner $I^{1}$, Springer $C^{1}$, Pschebezin $F^{1}$, Altmann $R^{1}$, Arzt $W^{1}$ Institut 1 Kepleruniversitätsklinikum, Linz/AT

DOI 10.1055/s-0037-1606903

Problemstellung Hereditäre neuromuskuläre Erkrankungen stellen eine äuBerst heterogene Gruppe dar, betreffend sowohl die genotypische als auch die phänotypische Ausprägung. Die Tochter der Patientin im hier vorgestellten Fall ist Trägerin einer seltenen sporadisch aufgetretenen Mutation im DYNC1H1-Gen, die zu einem variablen Phänotyp - laut Literatur von einer proximalen spinalen Muskelatrophie bis zu einer Charcot-Marie-Tooth-Erkrankung reichend - führt. Die beim Feten pränatal diagnostizierte Variante im DYNC1H1Gen wurde bis 2014 erst einmal bei einer Patientin beschrieben.

Patienten und Methode Eine 30-jährige Zweitgravida wurde in der 21+3 SSW zum Organscreening am Institut für Pränatalmedizin des Kepleruniversitätsklinikums Linz vorstellig. Hier fielen eine permanente Extensionsstellung der unteren Extremität im Sinne von Genua recurvata und Talipes beidseitig auf. Weiters wurden die Händchen während der gesamten Untersuchung immer nur in Fauststellung („clenched fists“), die Unterarme ausschließlich flektiert gesehen. Zur weiteren Abklärung wurde eine Amniozentese durchgeführt. Hier konnte eine de novo Mutation im DYNCH1H1-Gen (c.791G>A (p. R264Q)) nachgewiesen werden, die von einer proximale spinale Muskelatrophie bis zu einer axonalen Charcot-Marie-Tooth-Erkrankung führen kann. Nach 
engmaschigen Kontrollen wurde in der $38+0$ SSW die primäre Sectio durchgeführt.

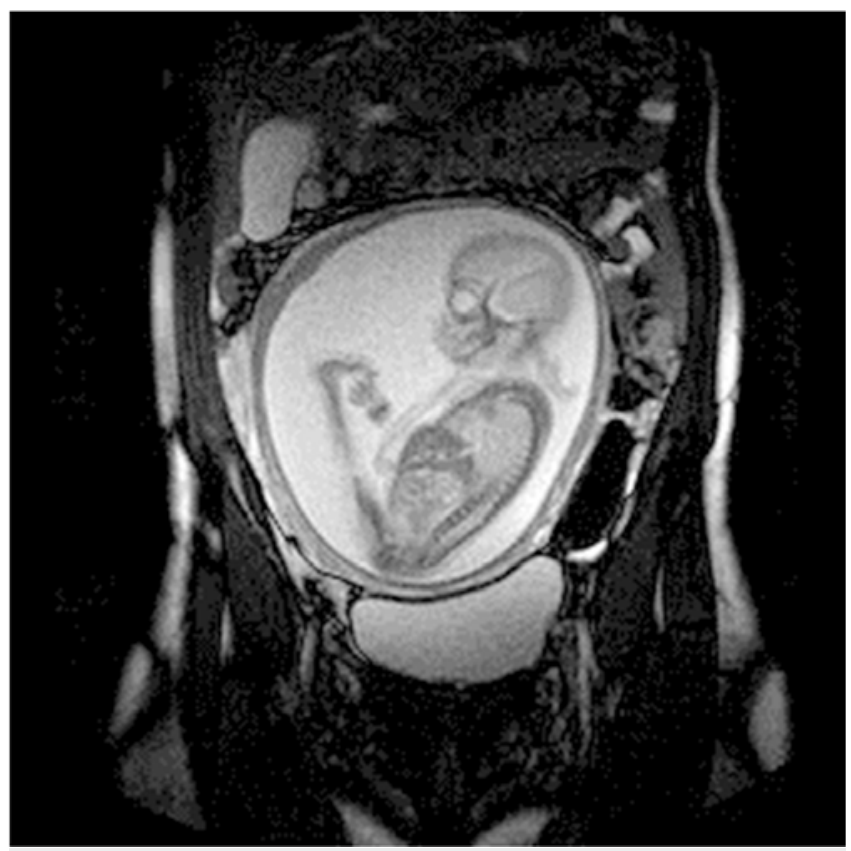

Abb. 1 MRT- Bild Extensionsstellung der unteren Extremität

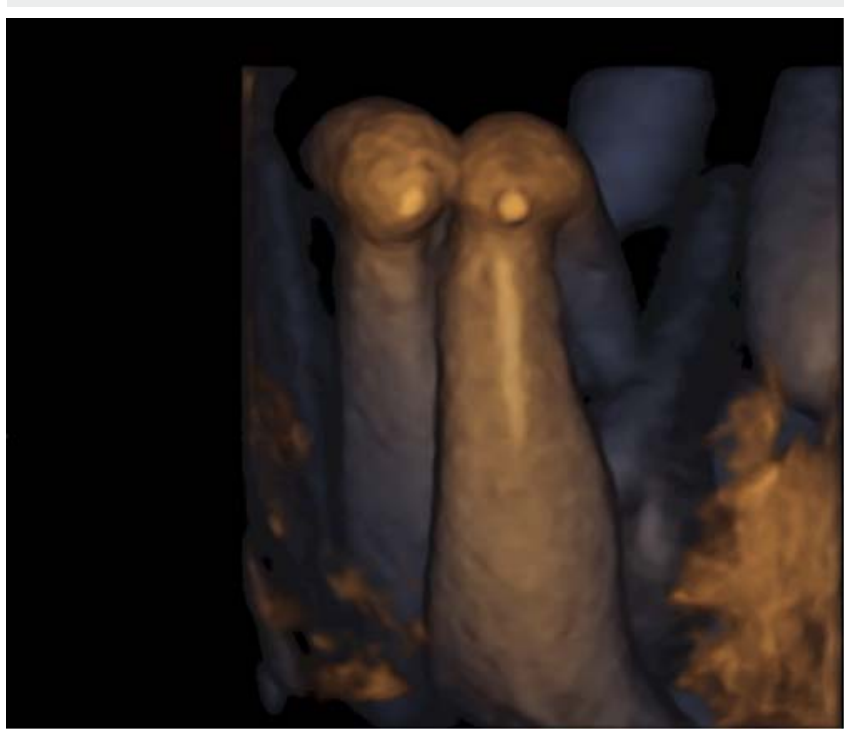

Abb. 2 3D- Ultraschall Genua recurvata

Ergebnis Im Rahmen der ersten Untersuchungen konnten die pränatal gestellten Verdachtsdiagnosen (Genua recurvata, Gelenkskontrakturen) bestätigt werden.

Schlussfolgerung Obwohl bei den meisten neuromuskulären Erkrankungen keine spezifischen Auffälligkeiten im Ultraschall zu beobachten sind, sollte bei suspektem Bewegungsmuster des Feten an die heterogenen Formen einer neuromuskulären Erkrankung gedacht werden. Die sonografischen Befunde können eine Polyhydramnie, verminderte oder abnorme fetale Bewegungsmuster, auffällige immer gleichbleibende Gelenksstellung, eine Zeltmundform und/oder eine grenzwertige Ventrikelerweiterung umfassen.

\section{6: Gyn/Pränatal III}

\section{V6.001 Untersuchung des Elastografiemusters der Zervix zur Vorhersage einer Frühgeburt}

Autoren Oturina $\mathrm{V}^{1}$, Hammer $\mathrm{K}^{2}$, Möllers $\mathrm{M}^{2}$, Braun J², Falkenberg $\mathrm{M}^{2}$, Oelmeier de Murcia $\mathrm{K}^{2}$, Eveslage $\mathrm{M}^{3}$, Fruscalzo $\mathrm{A}^{4}$, Klockenbusch $\mathrm{W}^{2}$, $\underline{\text { Schmitz } \mathrm{R}^{2}}$

Institut 1 St. Marien-Hospital Borken, Borken/DE; 2 Universitätsklinikum Münster, Münster/DE; 3 Universität Münster, Münster/DE; 4 St. FranziskusHospital Ahlen, Ahlen/DE DOI 10.1055/s-0037-1606904

Problemstellung Untersuchung der Elastografiemusters der Zervix mittels transvaginaler Sonografie zur Vorhersage einer Frühgeburt.

Patienten und Methode In einer Fall-Kontroll-Studie wurden 30 Schwangerschaften mit einer Frühgeburt und 30 gestationsalter geparte Kontrollen mittels transvaginaler Sonografie untersucht. Es wurde die Zervixlänge bestimmt und eine Elastografiemessung durchgeführt (IU22 und EPIQ7, Philips Medical Systems, Andover, MA, USA). Anschließend wurden offline die maximalen Strain Ratio Werte für vier unterschiedliche Regionen (ROIs) im Bereich der vorderen Muttermundslippe berechnet (SR1-SR4) (QLAB, Advanced Quantification Software, Release 10.2, Philips Medical Systems). Zur Überprüfung der Zuverlässigkeit der Elastografiemessungen wurde die Inter- und Intraobservervariabilität überprüft.

Ergebnis Die Elastografiemessung konnte mit einer hohen inter- und intraobserver Realibiltät durchgeführt werden (Intra-Class Correlation Coefficienten >0,96). Die Werte des Strain Ratio Maximums zwischen zwei parallel positionierten ROls im Bereich der mittleren vorderen Muttermundlippe (SR4) waren in der Gruppe der Schwangeren mit einer Frühgeburt gegenüber den Kontrollen erhöht (1,62 vs. 1,16; $p<0,0001)$. Die Vorhersagewahrscheinlichkeit der Zervixländenmessung (Area Under the Curve, AUC 0,74) und Elastografiemessung (SR4, AUC 0,83) für eine Frühgeburt waren vergleichbar. Die Kombination beider Messmethoden zeigte eine exzellente Vorhersagewahrscheinlichkeit (AUC 0.88). Es konnte ein Zusammenhang zwischen dem Auftreten eines speziellen Elastografiemusters („Kuhfleckenmuster“) und der Wahrscheinlichkeit einer Frühgeburt beobachtet werden.

Schlussfolgerung Die Berechnung des gewebespezifischen Strain Ratio Maximums mittels transvaginaler Elastografie Messung im Bereich der vorderen Muttermundlippe (SR4) korreliert mit der Wahrscheinlichkeit für eine Frühgeburt. Zukünftig könnte die Elastografiemessung als Zusatzparameter zur Zervixlängenmessung genutzt werden.

\section{V6.002 Schwangerschafts-Outcome bei feto-fetalem Transfusionssyndrom mit verkürzter Zervix $\leq 15 \mathrm{~mm}$}

Autoren Ortiz J ${ }^{1}$, Eixarch $\mathrm{E}^{2}$, Micheletti $\mathrm{T}^{2}$, Torres $\mathrm{X}^{2}$, Lobmaier $\mathrm{S}^{1}$, Martinez $\mathrm{J}^{2}$, Gratacós $\mathrm{E}^{2}$

Institut 1 Klinikum rechts der Isar, München/DE; 2 BCNatal, Barcelona/ES DOI 10.1055/s-0037-1606905

Problemstellung Analyse der Auswirkung einer verkürzten Zervix $\leq 15 \mathrm{~mm}$ auf das Schwangerschafts-Outcome bei Zwillingsschwangerschaften mit feto-fetalem Transfusionssyndrom (FFTS).

Patienten und Methode Retrospektive Analyse von 474 Zwillingsschwangerschaften mit FFTS, die mittels Laser behandelt wurden. Ultraschall- und chirurgische Parameter sowie das perinatale Outcome wurden zwischen 2 Gruppen ( $\leq 15 \mathrm{~mm}$ vs. $>15 \mathrm{~mm}$ ) verglichen. Außerdem wurde das Management (abwartendes Vorgehen vs. Cerclage) in der Gruppe mit Zervix $\leq 15 \mathrm{~mm}$ untersucht.

Ergebnis 30 (6,3\%) Patientinnen hatten eine Zervixlänge $\leq 15 \mathrm{~mm}$ vor dem Eingriff. Diese Gruppe zeigte eine geringere Zeitspanne zwischen Operation und Geburt, ein niedrigeres Gestationsalter bei der Geburt, eine höhere Früh- 
geburtsrate vor 32+0 SSW und eine niedrigere neonatale Überlebensrate von mindestens einem Zwilling. Die Abort- und vorzeitige Blasensprungrate $(<32$ +0 SSW) war vergleichbar zwischen den Gruppen. 21 Patientinnen erhielten keinen Zervixeingriff und 9 wurden mittels Cerclage behandelt. Es gab keinen Unterschied bzgl. des perinatalen Outcomes zwischen diesen Gruppen.

Schlussfolgerung Eine präoperative Zervixlänge $\leq 15 \mathrm{~mm}$ bei Zwillingsschwangerschaften mit FFTS korreliert aufgrund erhöhter Frühgeburtlichkeit und niedriger neonataler Überlebensrate mit einer schlechteren Prognose. Die Platzierung einer Cerclage scheint das Schwangerschafts-Outcome nicht zu verbessern.

\section{V6.003 Assoziation von Kurzzeitvariation und Doppler-Parametern mit dem perinatalen Outcome}

Autoren Stumpfe $\mathrm{F}^{1}$, Pretscher $\mathrm{J}^{1}$, Raabe $\mathrm{E}^{1}$, Baier $\mathrm{F}^{1}$, Kehl $\mathrm{S}^{1}$, Schneider $\mathrm{M}^{1}$, Schmid $\mathrm{M}^{2}$, Mayr $\mathrm{A}^{1}$, Beckmann $\mathrm{M}^{1}$, Faschingbauer $\mathrm{F}^{1}$

Institut 1 Universität Erlangen, Erlangen/DE; 2 Universität Bonn, Bonn/DE DOI 10.1055/s-0037-1606906

Problemstellung Analyse des Zusammenhangs zwischen Kurzzeitvariation und Doppler-Parametern mit dem perinatalen Outcome bei Feten am Termin. Patienten und Methode In diese historische Kohortenstudie wurden „lowrisk" Einlingsschwangerschaften (AGA-Feten) mit geplanter vaginaler Geburt $\geq 37$ SSW, vorhandener Doppleruntersuchung (A. umbilicalis (UA), A. cerebri media (ACM), cerebroplacentare Ratio (CPR)) innerhalb von 72 Stunden vor Geburt sowie durchgeführtem computerisiertem CTG mit Kurzzeitvariation eingeschlossen. Mittels univariater Regressionsanalyse wurde der Zusammenhang zwischen Kurzzeitvariation und Doppler-Parametern mit dem perinatalen Outcome analysiert. Outcome-Parameter waren sekundäre Sectio, vaginal-operative Entbindung, sekundäre Sectio und vaginal-operative Entbindung bei pathologischem CTG, Notkaiserschnitte sowie Nabelschnur-pH $\leq 7,10$, 5-min-Apgar $<7$ und kombiniertes adverses perinatales Outcome (APO) (Notkaiserschnitt und/oder pH $\leq 7,10$ und/oder 5-Minuten-Apgar $<7$ ). Ergebnis 1008 Frauen wurden eingeschlossen. 778 (77,2\%) entbanden spontan, 133 (13,2\%) per sekundärer Sectio caesarea und 97 (9,6\%) durch vaginaloperative Entbindung. Für alle Dopplerparameter ergaben sich bei Notkaiserschnitten $(n=8 ; 0,8 \%)$ signifikante Unterschiede (UA MoM: $p=0,04 / A C M$ MoM: 0,03/CPR MoM: <0,01). Während sich bei sekundären Sectiones und vaginal-operativen Entbindungen insgesamt keine signifikanten Unterschiede zeigten, waren UA MoM bei sekundären Sectiones aufgrund pathologischen CTGs signifikant höher $(p=0,02)$. Feten mit niedrigem Apgar-Score zeigten signifikant niedrigere ACM MoM $(p=0,02)$. Hinsichtlich des Nabelschnur-pHs ergaben sich höhere UA MoM $(p=0,02)$. Feten mit APO zeigten höhere UA MoM $(p<0,01)$ und niedrigere CPR MoM $(p<0,01)$.

Für keinen der untersuchten Outcome-Parameter zeigte sich ein signifikanter Zusammenhang mit der Kurzzeitvariation.

Schlussfolgerung Während Doppler-Parameter mit den meisten OutcomeParametern einen Zusammenhang zeigten, scheint die Kurzzeitvariation bei „low-risk“ Feten am Termin kein Prädiktor für ungünstiges perinatales Outcome zu sein.

\section{V6.004 Anwendung der Oxford-CTG gestützten Kurzzeitvariation bei Feten mit später intrauteriner Wachstumsrestriktion}

Autoren Graupner $\mathrm{O}^{1}$, Ortiz J ${ }^{1}$, Schmidt $\mathrm{G}^{1}$, Müller $\mathrm{A}^{1}$, Lees $\mathrm{C}^{2}$, Lobmaier $\mathrm{S}^{1}$ Institut 1 Klinikum rechts der Isar, Technische Universität München, München/DE; 2 Imperial College London, London/UK

DOI 10.1055/s-0037-1606907

Problemstellung Late-onset IUGR- (intrauterine Wachstumsrestriktion) Feten haben ein schlechteres peri- und postpartales Outcome. Die Fokussierung auf das Flussprofil des umbilikal-arteriellen (UA) Dopplers ist hierbei für die fetale Überwachung nicht zuverlässig. Ziel dieser Studie ist es, den Nutzen der Oxford-CTG gestützten Kurzzeitvariation (STV) zur besseren Klassifizierung sowie
Vorhersage des peripartalen Outcome von late-onset IUGR-Feten zu untersuchen.

Patienten und Methode Das Studienkollektiv umfasst 87 late-onset SGA(small for gestational age) Feten, davon 52 mit Anzeichen einer späten IUGR $($ EFW $<3$. Perzentile oder EFW $<10$. Perzentile in Kombination mit pathologischen feto-maternalen Dopplerwerten), und 138 Kontrollfeten. Die Doppleruntersuchung beinhaltete den UA-Pulsatilitätsindex (PI), den MCA- (mittlere zerebrale Arterie) PI, die CPR (cerebro-plazentare Ratio) und den mittleren PI der Aa. uterinae (mUtA). Die Oxford-CTG-Aufnahmen wurden mithilfe der Sonicaid FetalCare Software zur Ermittlung der STV anhand des Dawes/Redman-Algorithmus analysiert.

Ergebnis Das mittlere Zeitintervall zwischen STV-Messung und Entbindung betrug 15 Tage in der IUGR-Gruppe und 26 Tage in der Kontroll- und SGAGruppe. Bezogen auf die STV zeigte sich kein statistisch signifikanter Unterschied zwischen der Kontroll- (11,2 ms), SGA- (11,2 ms) und IUGR- (10,5 ms) Gruppe. Nur in der Kontrollgruppe ließ sich eine signifikant positive Korrelation von STV und 5 bzw. 10 Minuten Apgar-Wert beobachten. In der IUGRGruppe zeigte sich keine signifikante Korrelation zwischen STV- und Dopplerbefunden.

Schlussfolgerung Late-onset IUGR ist eine anspruchsvolle Diagnose. Unsere Ergebnisse zeigen, dass es keinen signifikanten Unterschied in den STV-Werten zwischen Kontrollen, late-onset SGA und IUGR Feten gibt. Weitere prospektive Untersuchungen sind erforderlich, um die Rolle der STV als sinnvolle Überwachungsmethode für late-onset IUGR Feten zu untersuchen.

\section{F 2 Mesenchymale Plazentadysplasie}

Autoren Delius $\mathrm{M}^{1}$, Hübener $\mathrm{C}^{1}$, Hasbargen $\mathrm{U}^{1}$, Flemmer $\mathrm{A}^{2}$, Mahner $\mathrm{S}^{2}$, Meuter $\mathrm{A}^{1}$

Institut 1 LMU München, München/DE; 2 LMU, München/DE DOI 10.1055/s-0037-1606908

Problemstellung Die mesenchymale Plazentadysplasie (PMD) ist eine seltene Zottenentwicklungsstörung, die zu einer molenartigen Plazentaanlage führt. Zytogenetisch besteht, im Gegensatz zur Partialmole, in der Regel ein diploider Chromosomensatz. Als mögliche Ätiologie wird ein androgenetisch biparentales Mosaik beschrieben, insbesondere im Zusammenhang mit kindlichen Leberharmatomen. Der fetale Karyotyp ist demnach mehrheitlich weiblich. Neugeborene können klinisch unauffällig sein, jedoch ist das Risiko für ein IUGR/IUFT erhöht. PMD kann mit dem Beckwith-Wiedemann-Syndrom (BWS), solitären Omphalozelen und selten mit multiplen mesenchymalen Leberhamartomen assoziiert sein. Ein Zusammenhang mit maternalen Thrombosen ist beschrieben.

Patienten und Methode Eine 25-jährige G2P1 wurde mit V.a. Partialmole und singulärer Nabelschnurarterie in der 12. SSW überwiesen. Die Chorionzottenbiopsie zeigte ein unauffälliges Karyogramm mit diploidem Chromosomensatz (46XX). Die Ätiologie der Plazentaauffälligeit konnte in der Schwangerschaft nicht eindeutig diagnostiziert werden. Bei Größenzunahme der Plazenta und progredientem IUGR wurden wöchentliche sonografische Kontrollen durchgeführt. Bei 33+0 SSW eine Lungenreifeinduktion initiiert.

Ergebnis Bei zunehmendem Plazentawachstum, IUGR, vaginaler Blutung und Wehentätigkeit wurde bei 33+1SSW die sekundäre Sectio durchgeführt. Es zeigte sich eine übergroße Plazenta $(945 \mathrm{~g})$ mit blasig und zystisch aufgetriebenen Stammzotten- und Deckplattengefäßen, teilweise mit Prolaps in die Nabelschnur, thrombotischen Gefäßverschlüssen sowie ausgedehnten peripheren Plazentainfarkten. Nachdem bei der Mutter postpartal eine Ovarialvenenthrombose und eine Lungenembolie auftrat und bei dem Neugeborenen multiple mesenchymale Leberhamartome und eine hämolytische Anämie festgestellt wurden, wurde, kongruent mit der Plazentahistologie die Diagnose der mesenchymalen Plazentadysplasie gestellt.

Schlussfolgerung Bei sonografischem V.a. Partialmole der Plazenta sollte PMD trotz ihrer Seltenheit, differentialdiagnostisch in Erwägung gezogen werden. 


\section{V6.005 Größe des fetalen Thymus bei Schwangeren mit diabetischen Erkrankungen}

Autoren Möllers $M^{1}$, Dörnemann $R^{1}$, Koch $R^{2}$, Möllmann $U^{1}$, Falkenberg $M^{1}$, Klockenbusch $\mathrm{W}^{1}$, Schmitz $\mathrm{R}^{1}$

Institut 1 Universitätsklinik Münster, Münster/DE; 2 Uniklinikum Münster, Münster/DE

DOI 10.1055/s-0037-1606909

Problemstellung Das Ziel der Studie war die Bestimmung der fetalen Thymusgröße bei diabetischen Schwangeren im Vergleich zu normalen Schwangeren. Patienten und Methode Die fetale Thymusgröße wurde retrospektiv bestimmt anhand von sonografischen Bildern von 161 Schwangeren mit Diabetes und 161 Schwangeren ohne Komplikationen, welche einander anhand des Gestationsalters zugeordnet wurden. Der anteroposteriore Thymusdurchmesser und der intrathorakale mediastinale Durchmesser wurden gemessen und ihr Quotient errechnet [(Thymus-Thorax-Ratio (TT-Ratio)]. Zusätzlich bildeten wir den Quotienten aus dem anteroposterioren Thymusdurchmesser und dem Kopfumfang, genannt Thymus-Kopf-Ratio (TH-Ratio). Die Diabetes-Gruppe wurde weiter in drei Subgruppen unterteilt: 1. diätetisch eingestellter Gestationsdiabetes, 2. insulinpflichtiger Gestationsdiabetes und 3. präexistenter Diabetes mellitus.

Ergebnis Die TT-Ratio und die TH-Ratio waren in der Diabetes-Gruppe niedriger als in der Kontroll-Gruppe (jeweils $p<0,001$ ). Auch in allen drei DiabetesSubgruppen waren die TT- und die TH-Ratio jeweils niedriger $(p<0,001$ für alle drei Gruppen).

Schlussfolgerung Eine geringere Thymusgröße des Feten scheint mit einer diabetischen Erkrankung der Schwangeren assoziiert zu sein. Wir konnten zeigen, dass die fetale Thymusgröße als neuer prognostischer Parameter für einen mütterlichen Diabetes geeignet ist.

\section{V6.006 Familiäre Mutation des DONSON Gens als Ursache für das wiederholte Auftreten von mikrocephalem Zwergwuchs}

\author{
Autoren Schneider $\mathrm{U}^{1}$, de Vries $\mathrm{H}^{1}$, Fröber $\mathrm{R}^{1}$, Eichhorn $\mathrm{KH}^{2}$, Möller $\mathrm{A}^{3}$, \\ Vilser $\mathrm{C}^{1}$, Mentzel $\mathrm{HJ}^{1}$, Schulz $\mathrm{S}^{1}$ \\ Institut 1 Universitätsklinikum Jena, Jena/DE; 2 Gemeinschaftspraxis für \\ Pränatale Diagnostik, Weimar/DE; 3 Frauenarztpraxis, Pränataldiagnostik, \\ Stadtroda/DE \\ DOI 10.1055/s-0037-1606910
}

Patienten und Methode Auf der Anwendung neuer diagnostischer Methoden der Humangenetik liegt große Hoffnung bei der Abklärung komplexer Fehlbildungssyndrome, insbesondere, wenn Wiederholungsfälle eine familiäre Ursache nahelegen. Wir berichten den Fall einer 34-jährigen Patientin deren Kinder zweier aufeinanderfolgender Schwangerschaften innerhalb eines Jahres ein Fehlbildungssyndrom nahezu identischen Phänotypes mit schwerer IUGR, Mikrocephalie, Oligogyrie, fazialen Dysmorphien, distalen Extremitätenverkürzungen und Syndaktylien aufwiesen. Im ersten Fall erfolgte die Beendigung der Schwangerschaft. Bei unauffälliger Zytogenetik wurde auch ein Chr1q21.1-Deletionssysndrom (Thrombocytopenia-Absent-Radius) ausgeschlossen. Der paidopathologische Verdacht auf ein Roberts-Syndrom bestätigte sich nicht, eine hohe Mitomycin (MMC)-Sensitivität der Amnionzellkultur wies auf eine Fanconi Anämie hin. Auch diese konnte in der Mutationsanalyse nicht gesichert werden. Das zweite Kind wurde bei 38+1 SSW mit $2000 \mathrm{~g}$ lebend geboren. Hier war die MMC Sensitivität nicht gegeben, ebenso wurden ein Baller-Gerold-, Smitz-Lemli-Opitz-, 3-Phosphoglycerat-DehydrogenaseMangel ausgeschlossen und eine array-CGH blieb ohne wegweisende Befunde. Auffällig ist neben den morphologischen Entwicklungsstörungen ein kombinierter B- und T-Zell-Defekt. Im Verlauf fand sich eine compound heterozygote Mutation im DONSON Gen (c.1391G>A, p.Pro464Leu and c.1005$2 \mathrm{~T}>\mathrm{C}$ ) als ursächlich für das syndromale Geschehen. Das Kind ist im SPZ angebunden, etwa monatlich wegen Komplikationen in stationärer Betreuung, trägt eine Trachealkanüle und wird über PEG ernährt.
Schlussfolgerung Das erst 6 Monate nach Geburt des Kindes identifizierte ursächliche Gen wurde aktuell bei 29 Kindern mit mikrocephalem Zwergenwuchs beschrieben. Wie in der Forensik, so steht auch in der Perinatalmedizin die zunächst ergebnislos abgeschlossene Vordiagnostik unter dem Vorbehalt neuen Erkenntnisgewinns. (Reynolds JJ, et al. Nat Genet. 2017; 49:537 - 549)

\section{V6.007 Zwischen Angst und Hoffnung - Emotionales Erleben Schwangerer im Rahmen invasiver Pränataldiagnostik}

\section{Autoren Friebe-Hoffmann $\mathrm{U}^{1}$, Nonnenmann $\mathrm{E}^{1}$, Ebner $\mathrm{F}^{1}$, Lato $\mathrm{K}^{1}$,} Hönig DK ${ }^{2}$, Hütter $W^{3}$, Friedl $T^{1}$

Institut 1 Universität Ulm, Ulm/DE; 2 Universität Ulm, Ulm/DE; 3 Praxisklinik Frauenstrasse, Ulm/DE

DOI 10.1055/s-0037-1606911

Problemstellung Im Rahmen pränataler Diagnostik (PND) bedeutet ein invasiver Eingriff für schwangere Frauen oft ein nachweislich angstbehaftetes Ereignis, da in vielen Fällen ein auffälliger Ultraschallbefund zugrunde liegt oder aber die eigene Angst vor einer fetalen Erkrankung.

Patienten und Methode In der noch andauernden prospektiven Befragungsstudie wurde seit 04.2015 mittels eines 3-teiligen Fragebogens unter Berücksichtigung eines Physical Health Questionnaires (PHQ-4) neben anamnestischen Merkmalen das emotionale Erleben der invasiven PND von über 100 Schwangeren erfragt.

Ergebnis 84 Fragebögen konnten bislang vollständig ausgewertet werden. Es zeigte sich eine eindeutige Veränderung des emotionalen Erlebens der Patientinnen im Verlaufe der Diagnostik: 92\% der Schwangeren $(n=77)$ gaben Angst und Unsicherheit vor dem geplanten Eingriff (Median 4 auf einer Skala von 1 8), wobei $20 \%(n=17)$ starke bis sehr starke Angst (Score $6-8)$ empfanden. In $31 \%$ der Fälle $(n=26)$ lag ein auffälliger US-Befund vor, bei $18 \%(n=15)$ ein familiär erhöhtes Risiko für eine Chromosomenstörung; eine Altersindikation fand sich bei $58 \%$ der Schwangeren $(n=49)$. Nach dem Eingriff waren $75 \%$ ( $n=63$ ) der Patientinnen zunächst vor allem erleichtert, jedoch danach empfanden $88 \%(n=74)$ erneute Angst vor einem schlechten Untersuchungsergebnis (Median 5 auf einer Skala von 1 -8). Diese Angst manifestierte sich bei einer längeren Wartezeit. Glücksgefühle und Erleichterung nach Bekanntgabe des Ergebnisses waren eindeutig abhängig vom genetischen Befund.

Schlussfolgerung Bei den meisten Schwangeren scheint das emotionale Erleben im Rahmen invasiver PND abhängig von der ärztlichen Aufklärung und Führung mäanderförmig zu verlaufen. Das Verständnis um diesen Sachverhalt sollte bei der Betreuung werdender Eltern Berücksichtigung finden.

\section{V6.008 Kaiserschnittentbindung in Schwangerschaften mit infauster Prognose: ein ethisches Dilemma?}

Autoren Kyvernitakis I ${ }^{1}$, Arabin B ${ }^{2}$

Institut 1 Bürgerhospital Frankfurt, Frankfurt/DE; 2 Philipps-Universität

Marburg, Marburg/DE

DOI 10.1055/s-0037-1606912

Patienten und Methode Die Begleitung und Entbindung von Schwangerschaften mit infauster Prognose stellen eine besondere Situation für werdende Eltern und behandelnde Ärzte dar. Wir berichten über zwei Fälle mit Omphalozele, Kloakenexstrophie, Anusatresie und Wirbelsäulendefekten (OEIS Komplex) mit einer überlappenden body stalk Anomalie. Trotzdem wurde die Schwangerschaft fortgeführt und die Kinder via Sektio entbunden.

1. Bei einer 39-jährigen Erstgravida nach ICSI wurde in der 12. SSW sonografisch ein OEIS festgestellt. Die Nabelschnur war extrem verkürzt im Sinne einer body stalk-Anomalie. Die Chromosomenanalyse war unauffällig (46xx). Obwohl die Eltern über die infauste Prognose aufgeklärt wurden, wünschten sie ein aktives Vorgehen. Die Sektio wurde in der 35. SSW bei V.a. AIS indiziert. Das Kind verstarb eine Stunde postpartal trotz aktiver Reanimationsmaßnahmen. 
2. Bei einer 24-jährigen G3 P0 zeigte sich im Rahmen des 1. Screenings bereits ein OEIS Komplex mit überlappender body stalk Anomalie. Der Chromosomensatz war 46XX. Nach eingehender interdisziplinärer Aufklärung wünschte das Ehepaar aus religiösen Gründen die Fortführung der Schwangerschaft und ein aktives Vorgehen. In der 38. SSW wurde eine Sektio durchgeführt und sogar eine EXIT-Prozedur durchgeführt. Das Kind verstarb am 1. postpartalen Tag.

Schlussfolgerung Es bedarf zeitweise mehr Mühe, Eltern eine rational vernünftige Entscheidung zur vaginalen Entbindung aufgrund einer infausten Prognose nahezulegen, als eine Indikation zum Kaiserschnitt zu stellen. Die Aussichtslosigkeit wird eher verschleiert, wenn die Pränataldiagnostik nicht in geburtsmedizinische Prozesse eingegliedert wird. Auch wenn Eltern sich nicht zu einem Abbruch entscheiden können, bleibt die Option eines „nicht aggressiven Managments“.

\section{V6.009 Prospektive Analyse von PIGF, sFlt-1 und PAPP-A im 3. Trimenon für die Prädiktion der late-onset fetalen Wachstumsretardierung}

Autoren Birdir $C^{1}$, Droste $L^{2}$, Fox $L^{2}$, Schmidt $B^{3}$, Frank $M^{3}$, Köninger $A^{2}$, Kimmig $R^{2}$, Wimberger $P^{1}$, Gellhaus $A^{2}$

Institut 1 Technische Universität Dresden, Dresden/DE;

2 Universitätsfrauenklinik Essen, Essen/DE; 3 Institut für Medizinische

Informatik, Biometrie und Epidemiologie, Essen/DE

DOI 10.1055/s-0037-1606913

Problemstellung Ist eine Prädiktion der late-onset fetalen Wachstumsretardierung durch eine prospektive Analyse der maternalen Serumwerte von PIGF, sFlt-1 und PAPP-A im 3. Trimenon möglich?

Patienten und Methode Es wurden maternale Serumwerte von PIGF, sFlt-1 und PAPP-A zwischen 28+1 und 40+0 Schwangerschaftswochen bei 22 Fällen mit fetaler Wachstumsretardierung und 676 Kontroll-Fällen mithilfe des ELISA (B.R.A.H.M.S KRYPTOR ${ }^{\circledR}$ ) prospektiv untersucht. Die Messungen wurden für das maternale Alter, Gestationsalter und BMI in logistischer Regressionsanalyse adjustiert. Eine ROC-Analyse wurde durchgeführt.

Ergebnis Es konnte signifikante Veränderungen der sFlt-1, PIGF und sFlt-1/ PIGF Quotient bei late-onset fetaler Wachstumsretardierung beobachtet werden. Die ROC-Analyse des sFlt-1/PIGF-Quotienten zeigte eine AUC von 0,70 (95\% Cl) mit einem cut-off von 63,6 (Sensitivität 50,0\%, Spezifität 93,9\%). Schlussfolgerung Eine Prädiktion der late-onset fetalen Wachstumsretardierung ist mit sFlt-1/PIGF Quotient im 3. Trimenon möglich.

\section{11: Gyn/Pränatal IV}

\section{V11.001 Die Kombination aus Afamin und 3D-Plazentabettdurchblutung im Ersttrimesterscreening verbessert die Vorhersage von Präeklampsie in niedrig-risiko Schwangerschaften}

Autoren Tramontana $A^{1}$, Hafner $E^{1}$

Institut 1 Donauspital Wien, Wien/AT

DOI 10.1055/s-0037-1606914

Problemstellung Das Ziel der Studie war, zum Zeitpunkt des Ersttrimesterscreenings bei gesunden Schwangeren und bei Schwangeren mit Gestationshypertonie, Präeklampsie, Gestationsdiabetes, intrauteriner Wachstumsretardierung und Frühgeburtlichkeit, die Bestimmung von Afaminserumwerten und ihrer Korrelation mit der 3D-Plazentabettdurchblutung, um zu untersuchen, ob die Kombination der Werte die Vorhersage dieser Schwangerschaftskomplikationen in einem Kollektiv mit niedrigem Risiko verbessert.

Patienten und Methode In dieser retrospektiven Fall-Kontrollstudie wurden 984 Patientinnen eines Kollektivs mit niedrigem Risiko zum Zeitpunkt des routinemäßigen Ersttrimesterscreenings zwischen der 11.-14. Schwangerschaftswoche untersucht. Schwangere mit pathologischem Outcome (Gesta- tionshypertonie $n=76$, Präeklampsie $n=33$, Gestationsdiabetes $n=170$, intrauterine Wachstumsretardierung $n=91$, Frühgeburtlichkeit $n=39$ ) wurden mit gesunden Kontrollen verglichen. Die Kombination von Afamin mit der 3DPlazentabettdurchblutung wurde auf ihren zusätzlichen prognostischen Wert zur Verbesserung der Vorhersage dieser Schwangerschaftskomplikationen getestet.

Ergebnis Signifikante Unterschiede von Afamin gab es in den Gruppen: Präeklampsie $(P=0,0214)$ und Gestationsdiabetes $(P=0,0074)$; Signifikante Unterschiede der Plazentabettdurchblutung gab es in den Gruppen: Gestationshypertonie $(P=0,0029)$, Präeklampsie $(P=0,0042)$, intrauterine Wachstumsretardierung $(P=<0,0001)$ und Frühgeburtlichkeit $(P=0,0306)$; In der Präeklampsiegruppe wurde ein signifikanter prognostischer Wert für Afamin $(O R=1,0197, P=0,0249)$ und die Plazentabettdurchblutung $(O R=0,9235$, $\mathrm{P}=<0,0001)$ festgestellt. Die Kombination dieser Werte konnte die Präeklampsievorhersage signifikant verbessern (AUC $=0,818$ ).

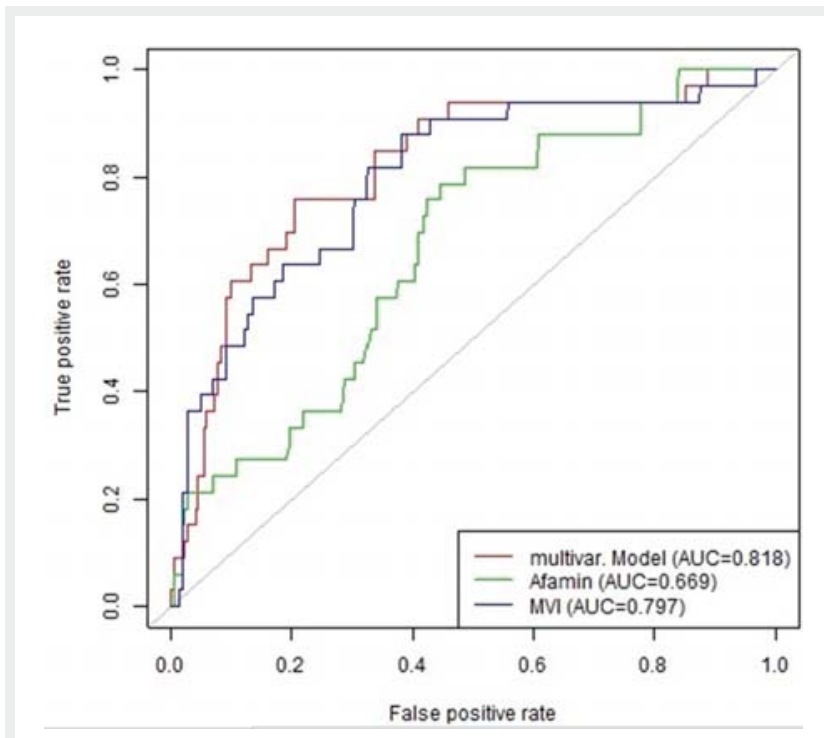

Abb. 1 ROC curve PE vs. Controls

Präeklampsievorhersage im Ersttrimesterscreening: ROC-Kurve für Afamin, Plazentabettdurchblutung und die Kombination aus Afamin und Plazentabettdurchblutung

Schlussfolgerung Afaminserumwerte in Kombination mit der 3D-Plazentabettdurchblutung zum Zeitpunkt des Ersttrimesterscreenings verbessern die Vorhersage von Präeklampsie in Schwangerschaften mit niedrigem Risiko und ermöglichen ein effizientes Screening zur Entdeckung von Risikoschwangerschaften für fetale und maternale Schwangerschaftskomplikationen bereits im ersten Trimenon.

\section{V11.002 Erfolgreiche pränatale Therapie einer genetisch bedingten Entwicklungsstörung durch Verabreichung eines rekombinanten Proteins ins Fruchtwasser}

Autoren Faschingbauer $\mathrm{F}^{1}$, Wohlfart $\mathrm{S}^{2}$, Schneider $\mathrm{P}^{3}$, Kirby $\mathrm{N}^{4}$, Beckmann $\mathrm{M}^{1}$, Schneider $\mathrm{H}^{2}$

Institut 1 Universität Erlangen, Erlangen/DE; 2 Universitätsklinikum Erlangen, Erlangen/DE; 3 Universität Lausanne, Lausanne/CH; 4 Edimer

Pharmaceuticals, Cambridge/US

DOI 10.1055/s-0037-1606915

Problemstellung Die X-chromosomale hypohidrotische ektodermale Dysplasie (XLHED) ist charakterisiert durch Mangel an Zähnen, Haaren und Schweißdrüsen. Ursache sind Mutationen des Gens EDA, welches Ektodysplasin A1 
kodiert. Aufgrund der fehlenden Schweißdrüsen kann es zu lebensbedrohlichen Hyperthermie-Episoden kommen.

Patienten und Methode Bei zwei Gemini-Feten und einem Einling mit XLHED wurde im Rahmen individueller Heilversuche mittels Amniozentese rekombinantes Fc-Ektodysplasin (Fc-EDA), das mithilfe von Fc-Rezeptoren im fetalen Darm transzytotisch in den fetalen Blutkreislauf aufgenommen werden könnte, mehrmals (Gemini, SSW 26 und 31) bzw. einmalig (Einling, SSW 26) ins Fruchtwasser appliziert. Zuvor wurde Fc-EDA bereits im Tiermodell, sowie in Studien an Erwachsenen und Neugeborenen evaluiert und für sicher befunden.

Ergebnis Die pränatale Verabreichung von Fc-EDA gelang jeweils komplikationslos. Zu verschiedenen Zeitpunkten danach entnommene Blutproben der Schwangeren gaben keinen Hinweis auf einen transplazentaren Übertritt des Proteins. Die Entbindung der Zwillinge erfolgte in der 33. SSW bei PROM durch Sectio (Geburtsgewichte 1.705 bzw. 1.615 g); der Einling kam in der 39. SSW mit $3.460 \mathrm{~g}$ Geburtsgewicht spontan zur Welt. Bei keinem der drei Kinder wurden Nebenwirkungen der Behandlung beobachtet. Konfokale LaserScanning-Mikroskopie der Fußsohlen zeigte jeweils eine normale Schweißdrüsenzahl. Pilocarpin-Schweißtests im Alter von 5 Monaten ergaben bei den Zwillingen eine normale, bei dem nur einmalig behandelten Kind eine reduzierte, aber ausreichende Schweißproduktion. Im Beobachtungszeitraum traten keinerlei Hyperthermie-Episoden auf.

Schlussfolgerung Pränatale Therapie mit Fc-EDA kann die Heilung einer bislang unheilbaren erblichen Entwicklungsstörung bewirken. Dieser weltweit erstmals durchgeführte Therapieansatz könnte auch auf andere genetische Erkrankungen übertragbar sein.

\section{V11.003 The maxillary gap sign at $11-13$ weeks - intra- and interobserver reliability in isolated cases}

\author{
Authors Lachmann $R^{1}$, Schilling $U^{1}$, Brückmann $D^{2}$, Brückmann $A^{1}$ \\ Institute 1 Fetal Medicine Centre, Dresden/DE; 2 Gemeinschaftspraxis \\ Brückmann, Erfurt/DE \\ DOI 10.1055/s-0037-1606916
}

Problemstellung To evaluate the reliability of the maxillary gap sign for detection of clef lip and palate (CLP) using the midsagittal view for measurement of nuchal translucency at 11-13 weeks of gestation in fetuses with isolated CLP.

Patienten und Methode This was a retrospective study of stored images of the mid-sagittal view of the fetal face at $11-13$ weeks' gestation in 5 cases of CLP and 100 normal controls. The affected cases were confirmed at 20-22 weeks. Two examiners blinded by the pregnancy outcome assessed independently the stored images twice. If identified the maxillary gap was measured. Ergebnis In all affected cases of CLP both sonographer identified the pictures independent correctly. There were no false-positive case. The gap diameter, if measurable was $>1.5 \mathrm{~mm}$ in all cases and in none of the normal controls. Schlussfolgerung The maxillary gap as described by Chaoui $R$ et al. is a reliable marker in cases with high risk for CLP with a low False Positive Rate and could increase the detection rate of CLP at $11-13$ weeks.

\section{V11.004 Artefakte bei der 3D/4D-Pränatalsonografie}

Autoren Miric Tesanic D' ${ }^{1}$, Merz $\mathrm{E}^{2}$

Institut 1 Poliklinika GynaeArs, Zagreb/HR; 2 Zentrum für Ultraschall und

Pränatalmedizin, Frankfurt a/M/DE

DOI 10.1055/s-0037-1606917

Einführung Die dreidimensionale Sonografie ermöglicht eine Darstellung des Feten in verschiedenen Darstellungsmodi. Für den Untersucher ergibt sich damit eine neue Sicherheit bei der Diagnosestellung und für die zukünftigen Eltern eine neue visuelle Erfahrung.

Ziel der Studie: Ziel dieser Studie war es, die Artefakte, die bei der 3D/4DUntersuchung auftreten können, einzugruppieren und deren Häufigkeit aufzuzeigen.
Material und Methode In den letzten fünf Jahren wurden 2349 Feten ohne Auffälligkeiten mit regelrechtem Wachstum und normaler Fruchtwassermenge in die Studie eingebracht. Alle Untersuchungen wurden mit dem Ultraschallgerät Voluson E8 (GE, Zipf, Austria) durchgeführt. Alle Kinder waren bei Geburt unauffällig.

Die bei der 3D/4D-Sonografie auftretenden Artefakte wurden je nach Auftreten in folgende Gruppen eingeteilt:

1. Bewegungsartefakte,

2. Schattenartefakte,

3. Amputationsartefakte infolge ungünstiger Volumenboxgröße,

4. Amputationsartefakte durch fehlerhafte Anwendung des elektronischen Skalpells,

5. Artefakte durch fehlerhafte Tresholdeinstellung und

6. Überlagerungsartefakte.

Resultate Insgesamt wurden bei 119 Untersuchungen ausgeprägte Artefakte gefunden: bei 39 Fällen zeigten sich Bewegungsartefakte, bei 25 Fällen traten Schattenartefakte auf, bei 19 Fällen fanden sich Amputationsartefakte infolge einer ungünstigen Volumenboxgröße, bei 15 Fällen Amputationsartefakte infolge fehlerhafter Anwendung des elektronischen Skalpells, bei 12 Fällen handelte es sich um eine fehlerhafte Thresholdeinstellung und bei 9 Fällen ergaben sich Artefakte durch Überlagerung.

Zusammenfassung Bei der 3D/4D-Ultraschalluntersuchung findet man unterschiedliche Artefakte, die zu einer Fehlbeurteilung führen können. Die rechtzeitige Erkennung eines Artefaktes während der Volumenaufnahme und die konsekutive Neuaufnahme eines Volumens verhindern eine Fehlbeurteilung des Feten.

\section{F 10 Fetales spontanes subdurales Hämatom in der 30. SSW bei Delta-Storage-Pool-Disease und Sichelzellenanämie: Sonografische Diagnose und Management}

Autoren lannaccone $A^{1}$, Darkwah Oppong $\mathrm{M}^{1}$, Kimmig $\mathrm{R}^{1}$, Köninger $\mathrm{A}^{2}$ Institut 1 Uniklinikum Essen, Essen/DE; 2 Uniklinikum Esse, Essen/DE DOI 10.1055/s-0037-1606918

Patienten und Methode Hintergrund: Die pränatale Diagnose eines subduralen Hämatoms ist selten, insbesondere ohne vorheriges Trauma.

Fallbericht: Wir berichten über eine 24-jährige Patientin IIG IP mit Sichelzellenkrankheit (HbSS).

Die Patientin wurde in der 23. SSW aufgrund Infekt-bedingter Krankheitsschübe stationär aufgenommen und bei einer Hämoglobinkonzentration $4 \mathrm{mg} / \mathrm{dl}$ wurden Erythroztenkonzentrate transfundiert.

Im Rahmen einer routinemäßigen sonografischen Verlaufskontrolle in der 29 +5 SSW zeigte sich beim Feten eine $15 \times 70 \mathrm{~mm}$ großer, vorwiegend echoarmer, subduraler Flüssigkeitsverhalt rechtshemismphärisch im Sinne eines subduralen Hämatoms. Hierdurch ergab sich eine deutliche Mittelinieverlagerung nach links, ein beginnender Hydrozephalus und eine erhöhte Flussgeschwindigkeit in der Arteria cerebri media.

Nach interdisziplinärer Diskussion mit Neurochirurgen und Neonatologen, folgte der Entschluss zur eiligen Sectio und anschließenden operativen Hämatom-Entlastung. Der Kaiserschnitt wurde komplikationslos durchgeführt mit Entwicklung eines Jungen (1600 g/Perc.: 70/Apgarwerte: 1'07/5'07/10'08). Die Diagnose wurde unmittelbar postnatal durch transkranielle Sonografie bestätigt. Es folgte die komplikationslose Entlastung des Hämatoms über eine rechts frontale Minitrepanation. Bei geringem Restbefund erfolgten regelmäßige sonografische Kontrollen. Zum Entlassungszeitpunkt zeigte sich kein nachweisbarer Rest. Neurologische Defizite bestanden nicht.

Die Patientin hatte einen komplizierten Verlauf mit mehrfachen Nachblutungen und Notwendigkeit operativer Revisionen.

Die Gerinnungsdiagnostik ergab eine Delta-Storage-Pool Disease bei Mutter und Kind, sowie ein Faktor X Mangel maternal. Bei dem Kind konnte ebenfalls eine HbSS Krankheit nachgewiesen werden. 


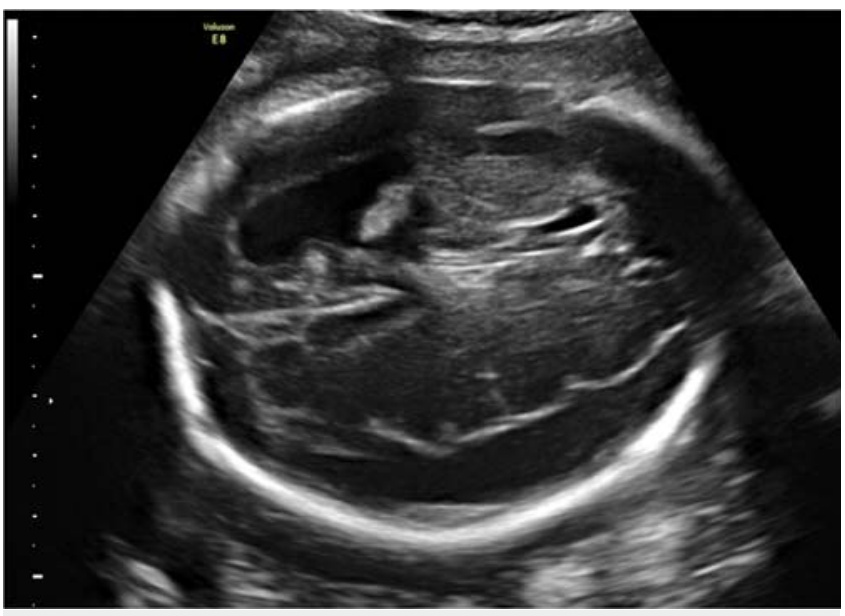

Abb. 1 Mittellinieverlagerung und beginnender Hydrozephalus

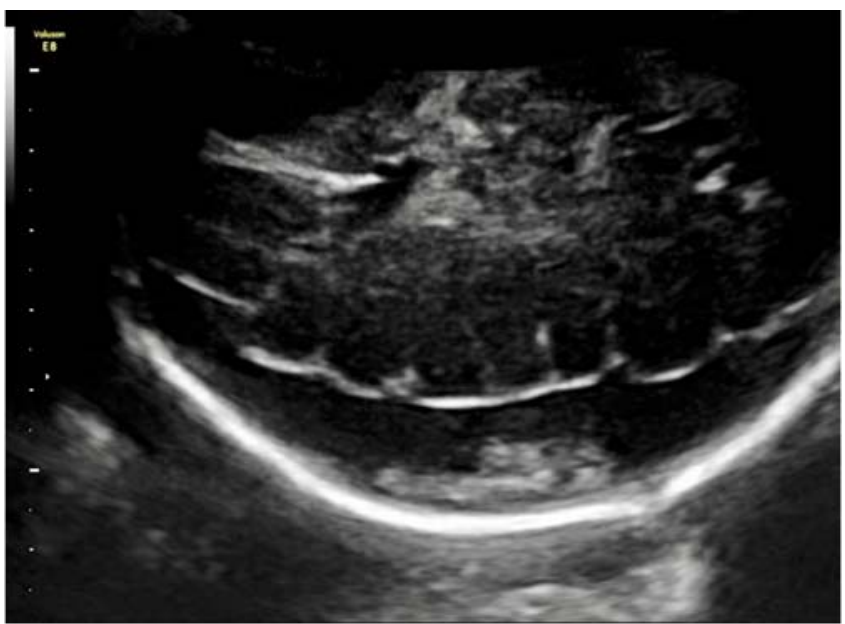

Abb. 2 Hämatom

Schlussfolgerung Fazit: Die intrauterine Diagnose von subduralen Hämatomen ist selten. In der Literatur finden sich einige Fallberichte, meistens liegen fetalen Traumata, Thrombozytopathien oder hypoxische Schäden und fetale Anämie vor (Ghi et al. 2003). Die sonografische Diagnosestellung ist gut möglich. Die multidisziplinäre Behandlung kann das Management und das Outcome verbessern.

\section{F 11 Cavum veli interpositi - Bedeutung dieses seltenen intrakraniellen Befundes für die pränatale Beratung}

Autoren Bosselmann $S^{1}$, Zierl $A^{2}$, Mielke $G^{1}$

Institut 1 Pränatalzentrum Stuttgart, Stuttgart/DE; 2 Olgäle, Stuttgart/DE DOI 10.1055/s-0037-1606919

Patienten und Methode Eine 33-jährige IIIG/IIP stellt sich zur sonografischen Fehlbildungsdiagnostik mit 21+6 SSW vor. Eigen- und Familienanamnese, der bisherige Schwangerschaftsverlauf und der aktuelle Befund sind unauffällig (Screeningsituation). Im weiteren Verlauf ( $32+5 \mathrm{SSW})$ kommt es zur Darstellung einer 10,6 $\times 5,5 \times 7,3 \mathrm{~mm}$ großen intrakraniellen Zyste im Bereich der Mittellinie unterhalb des Spleniums des Corpus callosum. Darüber hinaus ist die Neurosonografie unauffällig. Der Befund passt in seiner Erscheinung zu einem Cavum veli interpositi. Mit 37+0 SSW und postnatal ist der Befund reproduzierbar. Sowohl im pränatalen, als auch im postnatalen Verlauf gibt es keine weiteren Auffälligkeiten.

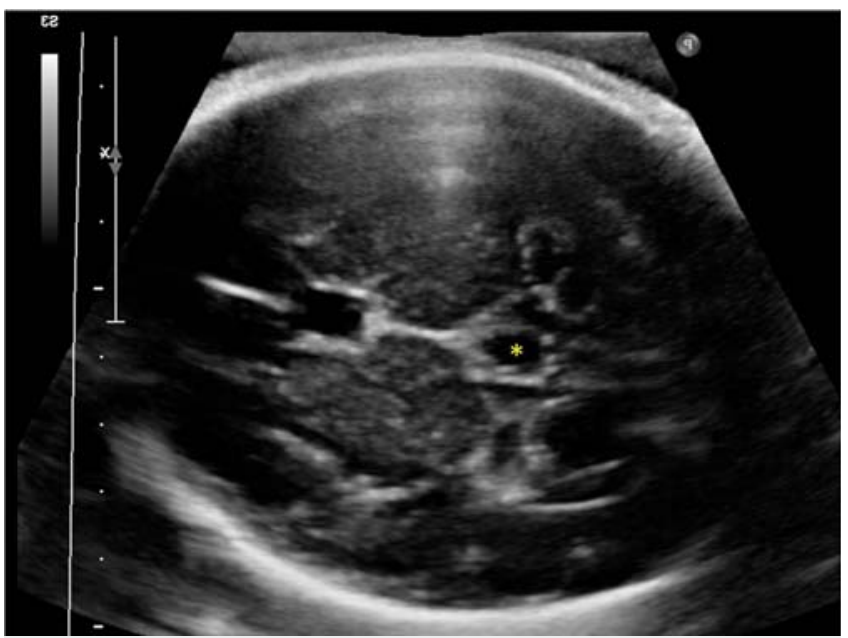

- Abb. 1 Cavum veli interpositi $\left({ }^{*}\right)$ im Axialschnitt (32+5 SSW)

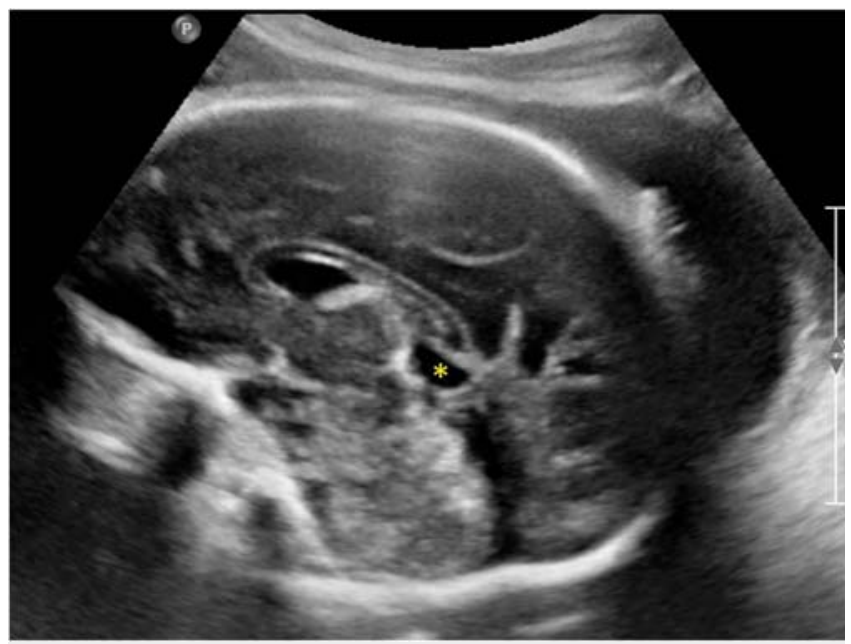

Abb. 2 Cavum veli interpositi $\left({ }^{*}\right)$ im Sagittalschnitt (32+5 SSW)

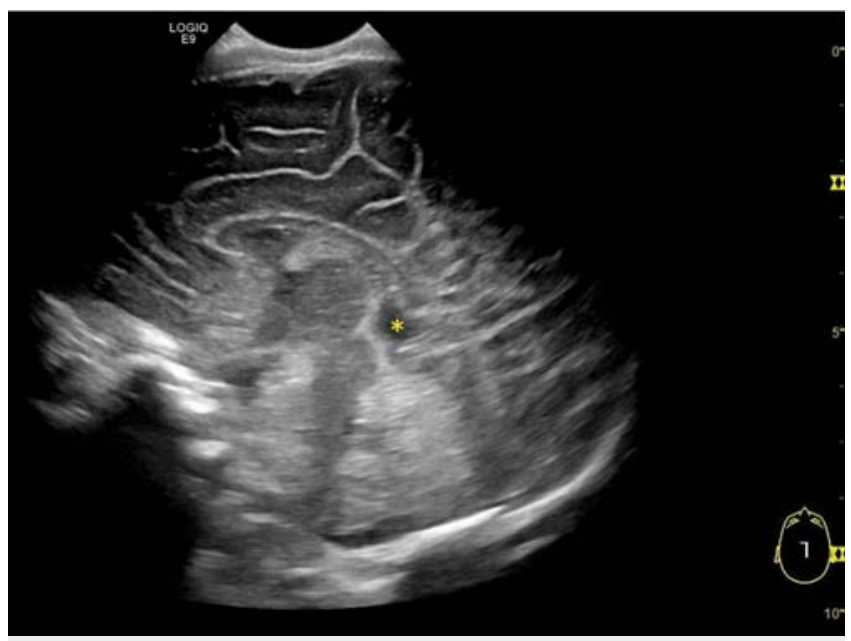

Abb. 3 Cavum veli interpositi $\left({ }^{*}\right)$ im Sagittalschnitt (4. Tag postnatal)

Schlussfolgerung Intrakraniell können Strukturen mit zystischer Erscheinung physiologisch sein (z. B. das Cavum septi pellucidi), als Variante mit untergeordneter klinischer Bedeutung (z. B. Plexuszysten) auftreten oder Ausdruck 
von Störungen mit erheblicher klinischer Bedeutung sein (z. B. Porencephalie, Vena-Galeni-Aneurysma).

Das Velum interpositum ist eine Invagination der Pia mater. Eine Erweiterung der Zisterne des Velum interpositum wird als Cavum veli interpositi bezeichnet und kann pränatalsonografisch als Zyste imponieren. Ihre Lage bedingt, dass das Splenium corporis callosi im Sagittalschnitt und der Interhemisphärenspalt frontal des Sulcus parieto-occipitalis im Axialschnitt als Leitstruktur fungieren können. Fälle wurden meist einzeln oder im Rahmen von kleinen Serien publiziert. Der Befund hat keinen Krankheitswert.

Die Diagnose insbesondere von Anomalien des Gehirns geht für die betroffenen Paare häufig mit einer besonders starken Beunruhigung einher. Die korrekte Einordnung von anatomischen Varianten ohne klinische Bedeutung kann sowohl unnötige Beunruhigung, als auch unnötige diagnostische Belastungen vermeiden.

\section{F 12 Thorakaler Fetus in Fet}

Autoren Götte $\mathrm{M}^{1}$, Seitz $\mathrm{C}^{2}$, Köhler $\mathrm{S}^{3}$, Enzensberger $C^{4}$, Axt-Fliedner $\mathrm{R}^{5}$ Institut 1 Zentrum für Frauenheilkunde und Geburtshilfe,

Universitätsklinikum Gießen \& Marburg, Abteilung für Pränatalmedizin, Gießen/DE; 2 Klinik für Kinderchirurgie, Universitätsklinikum Gießen \& Marburg, Marburg/DE; 3 Klinik für Frauenheilkunde und Geburtshilfe, Universitätsklinikum Gießen \& Marburg, Marburg/DE; 4 Zentrum für Frauenheilkunde und Geburtshilfe, Universitätsklinikum Gießen \& Marburg, Gießen/DE; 5 Universitätsklinikum Gießen und Marburg, Standort Gießen, Gießen/DE

DOI 10.1055/s-0037-1606920

Problemstellung Pränatale Diagnostik und postnatales Management bei Fetus-in-Fetu-Malformation.

Patienten und Methode Pränatale Vorstellung einer 29-jährigen IIG/OP in der 28. Schwangerschaftswoche mit einer dichorialen diamnialen Schwangerschaft.

Ergebnis Bei einem der Feten zeigte sich sonografisch eine thorakale parasitäre Zwillingsanlage mit zusätzlichen Extremitätenanlagen, einer weiteren Wirbelsäulenanlage sowie einer Omphalozele. Es präsentierte sich zusammenschauend das Bild einer Fetus-in-Fetu-Malformation des zweiten Geminus. Die Entbindung erfolgte in der 32+5 Schwangerschaftswoche aufgrund eines vorzeitigen Blasensprungs. Der Fet hatte ein Geburtsgewicht von 1980 Gramm, bei einem Apgar von 1-1-6. Im vier Wochen postpartum durchgeführten Kardio-MRT zeigte sich eine zusätzlich große einmündende Vene in den rechten Ventrikel. In der Twist-Angiografie konnte zudem die arterielle Versorgung des Phagen aus der großen Mesenterialarterie und der Arteria subclavia dargestellt werden. In der sechsten Lebenswoche erfolgte die erste operative Teilkorrektur mit Teilentfernung des Thorakophagus und seinen Anteilen. Anschließend erfolgte die intensivmedizinische Stabilisierung.

Schlussfolgerung Eine Fetus-in-Fetu-Malformation stellt eine seltene, meist benigne, Sonderform der siamesischen Zwillingsanlage dar, in der sich ein parasitärer Zwilling im Körper des Anderen befindet. In der Literatur sind nur circa 100 Fälle bekannt. Bezüglich des Zeitpunktes der Erstdiagnose und der pränatalen Entdeckungsrate, der Lokalisation und Ausprägung des Parasiten und der Behandlungsmöglichkeiten weist die Literatur starke Variationen auf. Die pränatale Diagnostik ist vor diesem Hintergrund ein wichtiger Faktor in Bezug auf die frühen diagnostischen und anschließend interventionellen Möglichkeiten im Rahmen dieser seltenen Zwillingsanomalie.

\section{F 13 Großes retrochoriales Hämatom in der 19. SSW im Rahmen eines HELLP-Syndroms und Schwangerschaftsverlauf}

\author{
Autoren Hamza $\mathrm{A}^{1}$, Takacs $\mathrm{Z}^{1}$, Solomayer $\mathrm{EF}^{1}$, Hamoud $\mathrm{BH}^{1}$, Sklavounos $\mathrm{P}^{1}$, \\ Pilch $\mathrm{J}^{1}$, Eichler $\mathrm{H}^{1}$, Meyberg-Solomayer $\mathrm{GC}^{1}$ \\ Institut 1 Universität des Saarlandes, Homburg/DE \\ DOI 10.1055/s-0037-1606921
}

Patienten und Methode Verlegung einer III. Gravida in der 18+5 SSW nach Sturz auswärts. Laborchemisch zeigte sich ein HELLP Syndrom entsprechend der Mississipi Klassifikation II. Bei der Aufnahme zeigte sich sonografisch ein $12 \times 9 \times 8 \mathrm{~cm}$ großes retrochoriales Hämatom, normotropher unauffälliger Fetus und eine Plazenta Prävia totalis. Die maternale Vitalparameter waren unauffällig. Die beiden vorausgegangenen Schwangerschaften wurden frühzeitig wegen einer schweren Präeklampsie per Sectio caesarea beendet. Mit der Patientin wurde eine Verlängerung der Schwangerschaft besprochen und eine Therapie mit Urbason (32 mg/d i.v.) begonnen. Hämostaseologisch bestand der V.a. eine Hyperfibrinolyse nach Faktor XIII Mangel mit dem laborchemischen Bild eines HELLP Syndroms. Nach Rücksprache mit der Hämostaseologie erfolgte zunächst eine antifibrinolytische Therapie und Substitution mittels $3 \mathrm{~g}$ Fibrinogen und 1250 IE Faktor XIII. Es kam zu einer hämostaseologischen Stabilisierung innerhalb von 6 Tagen und zu einer Verkleinerung des retrochorialen Hämatoms. Sonografisch zeigte sich der Verdacht auf eine Plazenta Accreta bei Z.n. 2 x Sectio. Die Schwangerschaft konnte bis zur 33. SSW fortgeführt werden. Bei vorzeitigem Blasensprung mit Amnioninfektionssyndrom wurde eine Sectio Caesarea durchgeführt. Aufgrund der Plazenta Prävia und Accreta, abgeschlossener Familienplanung und massive Blutung erfolgte die Hysterektomie im selber Sitzung. Das kindliche Outcome war unauffällig.

Schlussfolgerung Bei frühzeitigen HELLP Syndrom ist eine interdisziplinäre Zusammenarbeit zwischen den Gynäkologen und Hämostaseologen essentiell. Solche seltene Fälle sollten in Kliniken und Instituten mit entsprechender Expertise behandelt werden.

\section{F 14 Von der Darmdilatation zur Darmperforation: Verlauf eines Volvolus}

Autoren Kehl $\mathrm{S}^{1}$, Morhart $\mathrm{P}^{2}$, Carbon $\mathrm{R}^{2}$, Pretscher $\mathrm{J}^{1}$, Stumpfe $\mathrm{F}^{1}$, Beckmann $\mathrm{M}^{1}$, Faschingbauer $\mathrm{F}^{1}$

Institut 1 Universität Erlangen, Erlangen/DE; 2 Universitätsklinikum Erlangen, Erlangen/DE

DOI 10.1055/s-0037-1606922

Patienten und Methode Präpartaler Verlauf.

Eine 30-jährige Erstgebärende wurde mit 30+6 SSW erstmals wegen bereits extern seit längerem aufgefallener Darmdilatation vorgestellt. In der sonografischen Diagnostik fielen lediglich vereinzelte prominente und hyperechogene Darmschlingen auf, so dass eine Verlaufskontrolle vereinbart wurde. Bei dieser Untersuchung (33+1 SSW) wurde der Verdacht auf eine Darmperforation bei reichlich (mekoniumhaltigem) Aszites gestellt. Im Verlauf (33+3 SSW) wurde eine deutliche Zunahme des Aszites mit Zwerchfellverschiebung und Lungenkompression festgestellt, zudem eine progrediente Dilatation von Darmschlingen und des Magens. Aufgrund der Progredienz erfolgte die Sectio caesarea. Postnataler Verlauf.

Das Neugeborene wies ein grau-fahles Hautkolorit auf. Bei der Aszitespunktion des massiv ausladenden, gespannten Abdomens wurden direkt postnatal $60 \mathrm{ml}$ bräunliche Flüssigkeit entfernt, woraufhin die Beatmungsdrücke reduziert werden konnten. Präoperativ wurden kumulativ $280 \mathrm{ml}$ Aszites entfernt. In der apparativen Diagnostik wurde der Verdacht auf eine hohe Atresie/Stenose des Gastrointestinaltraktes mit einer Perforation gestellt. Intraoperativ stellten sich ein Volvolus ohne ersichtliche zugrunde liegende Fehlanlage und eine aseptische Peritonitis mit reichlich Mekonium in der Bauchhöhle dar. Es wurden circa $25 \mathrm{~cm}$ nekrotischer Dünndarm reseziert und ein endständiges Dünndarmstoma angelegt. Histopathologisch wurde eine Durchwanderungs- 
peritonitis mit teils chronisch-granulierender, teils fibrinös-eitriger Serositis, passend zu einem Volvolus beschrieben. Einige Wochen später konnte das Stoma rückverlagert werden und die Entlassung in die ambulante Betreuung erfolgen.

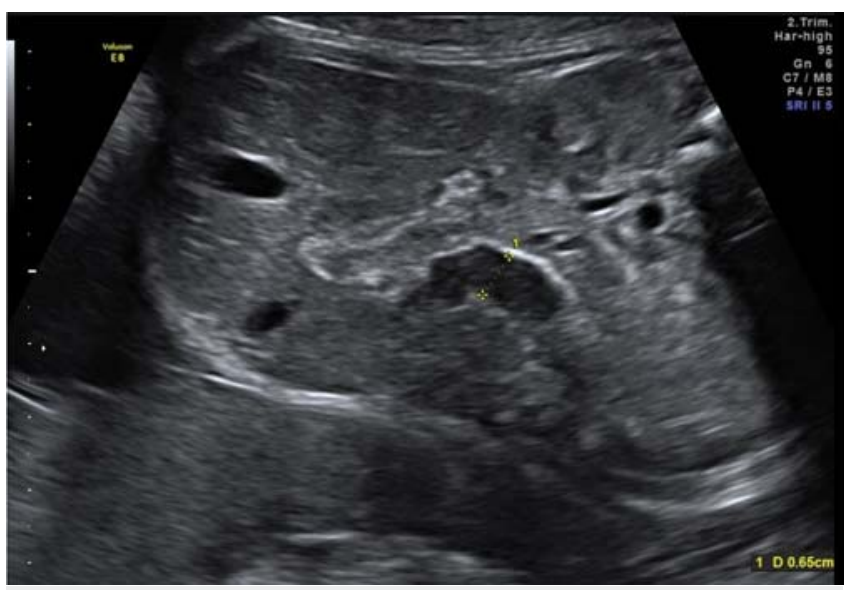

Abb. $130+6$ SSW

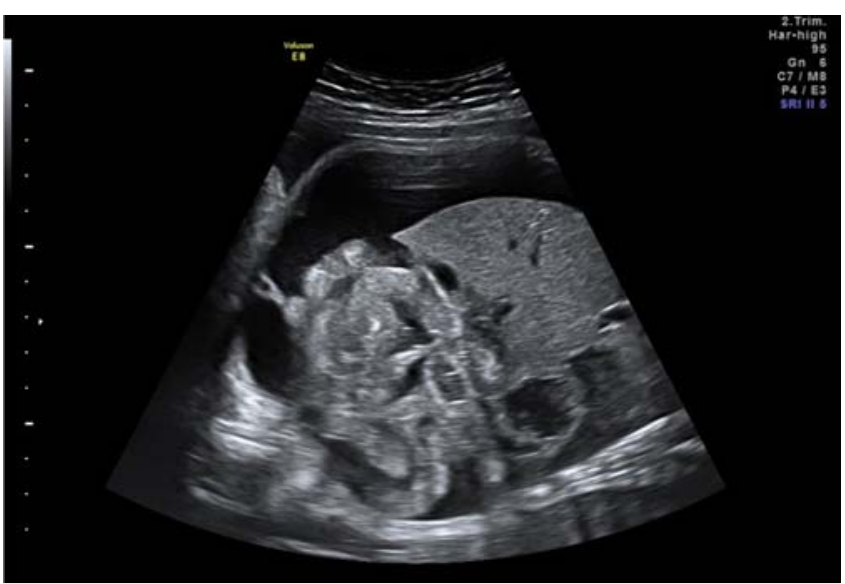

Abb. $233+1$ SSW

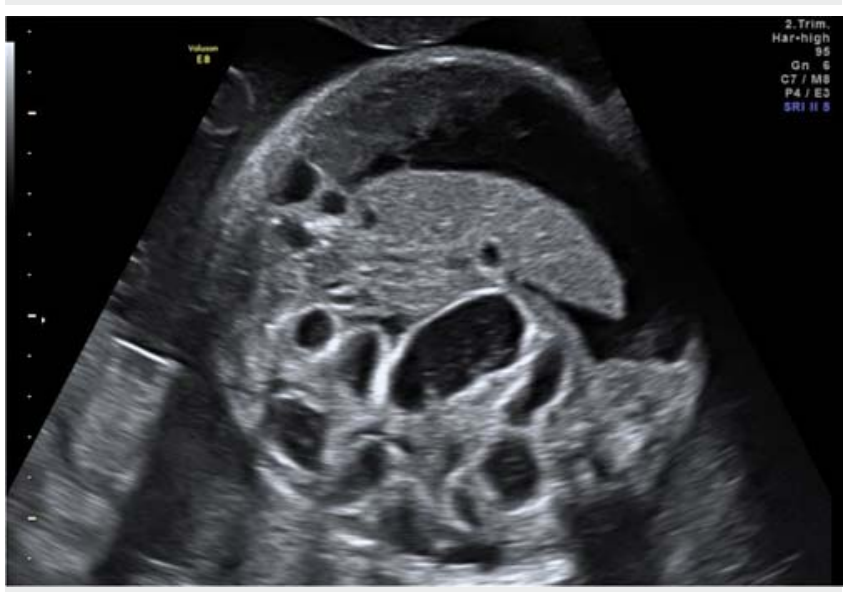

Abb. $333+3$ SSW

Schlussfolgerung Zusammenfassend handelt es sich hierbei um eine äußerst seltene Ursache einer hohen intestinalen Obstruktion mit hoher Mortalität und Morbidität. Die Diagnosestellung ist schwierig, aber entscheidend. Das Outcome hängt von Zeitpunkt des Auftretens und der Länge des betroffenen Darmabschnitts ab.

\section{V11.005 Desinfektion transvaginaler Ultraschallsonden - klinischer Vergleich einer automatischen Technik mittels ultravioletter Strahlung und einer manuellen Wischtuchmethode}

Autoren Schmitz ${ }^{1}$, Schmitz $\mathrm{R}^{1}$, Braun $\mathrm{J}^{1}$, Oelmeier de Murcia $\mathrm{K}^{1}$, Heese $\mathrm{S}^{1}$, Kossow $A^{1}$, Möllers $M^{1}$

Institut 1 Universitätsklinik Münster, Münster/DE

DOI 10.1055/s-0037-1606923

Problemstellung Da Keime über transvaginale Ultraschallsonden auf Patientinnen übertragen werden können, wird aktuell die Standardisierung der Desinfektionstechnik diskutiert.

In unserer Studie haben wir ein vollautomatisches Desinfektionsverfahren mit ultravioletter Strahlung im Vergleich zur herkömmlichen Wischtuchdesinfektion getestet.

Patienten und Methode Wir verglichen in einer prospektiven, kontrollierten Studie zwei Gruppen von je 160 Patientinnen die manuelle Wischtuchdesinfektion mittels Mikrozid ${ }^{\circledR}$ sensitive wipes (Schülke \& Mayr GmbH, Deutschland) mit der vollautomatische Desinfektion mittels Antigermix ${ }^{\circledR}$ AS1 (Germitec, Frankreich). Mikrobiologische Proben wurden steril vor und nach der Desinfektion von der gesamten Sonde entnommen und untersucht. Ergebnis Nach Auswertung der Ergebnisse kurz vor Abschluss der Studie waren vor Desinfektion 98\% der Proben kontaminiert. Nach Desinfektion durch den Antigermix ${ }^{\circledR}$ lag die Kontamination bei $42,0 \%$ und nach Tuchdesinfektion bei $42,1 \%$. Erreger von nosokomialen Infektionen wie Staphylococcus aureus und Enterococcus faecalis wurden durch beide Verfahren komplett entfernt. Gegen Keime der Vaginal- und Hautflora zeigte hingegen die Wischtuchdesinfektion mit 89,5\% eine bessere Wirksamkeit (Antigermix ${ }^{\circledR}$ 68,9\%). Eine Rekontamination mit Hautkeimen kann nicht ausgeschlossen werden. Umweltkeime und nicht fermentierende Bakterien, die größtenteils auf die Handhabung zurückzuführen sein könnten, wurden vor allem durch den Antigermix ${ }^{\circledR}$ beseitigt, spielen aber aufgrund der geringen Pathogenität eine untergeordnete Rolle.

Schlussfolgerung Antigermix ${ }^{\circledR}$ und Wischtuchdesinfektion zeigen beide eine sehr gute Wirksamkeit gegen pathogene Erreger. Bei den Verfahren ist eine adäquate Anwendung essentiell, um eine Kontamination nach Desinfektion zu vermeiden.

\section{13: Kontrastmittel}

\section{V13.001 CEUS-basierte Algorithmen für die Diagnose des hepatozellulären Karzinoms bei Risikopatienten - ESCULAP versus ACR CEUS-LI-RADS}

Autoren Schellhaas $B^{1}$, Pfeifer $L^{1}$, Görtz $R^{1}$, Neurath $M^{1}$, Strobel $D^{1}$ Institut 1 Universitätsklinikum Erlangen, Erlangen/DE

DOI 10.1055/s-0037-1606924

Problemstellung Ziel der Arbeit war die Untersuchung der diagnostischen Genauigkeit zweier Kontrastmittelultraschall-(CEUS-)basierter Algorithmen für die Diagnostik des hepatozellulären Karzinoms (HCC) bei Risikopatienten: ESCULAP (Erlanger Synopsis of Contrast-enhanced Ultrasound for Liver lesion Assessment in Patients at Risk) und ACR-CEUS-LI-RADSv.2016 (American College of Radiology CEUS-Liver Imaging Reporting and Data System).

Patienten und Methode Fokale Leberläsionen bei 120 Risikopatienten wurden mit CEUS untersucht. Die Leberläsionen wurden anhand ihrer Größe und ihres Kontrastmittelverhaltens in arterieller, portal-venöser und Spätphase klassifiziert. Es erfolgte eine Kategorisierung der Läsionen anhand beider CEUS-Algorithmen (ESCULAP und ACR CEUS-LI-RADSv.2016) unabhängig voneinander im direkten Vergleich. Für die Untersuchung der diagnostischen Genauigkeit der CEUS-basierten Diagnosen erfolgte ein Vergleich mit der Histologie als Goldstandard. 
Ergebnis Der Referenzstandard für die 120 Leberläsionen ergab 102 HCCs, 9 ICCs, und 9 Nicht-HCC-nicht-ICC-Läsionen. Die diagnostische Genauigkeit von CEUS für die Diagnose eines HCCs (ESCULAP-4, ESCULAP-V/LR-5, LR-5V) war signifikant höher mit ESCULAP versus ACR-CEUS-LI-RADS (95,1\%/62,7\%; $P<0,001)$. Sensitivität, Spezifität, positiver (PPW) und negativer prädiktiver Wert (NPW) für ESCULAP/ACR-CEUS-LI-RADS waren 95,1\%/62,7\%; 66,7\%/ $77,8 \% ; 94,2 \% / 94,1 \%$ und 70,6\%/26,9\%. Fusion der CEUS-LI-RADS-Kategorien LR-4 und LR-5 würde zu einer signifikanten Verbesserung von diagnostischer Genauigkeit, Sensitivität und NPV führen ohne Verlust an Spezifität.

Schlussfolgerung ESCULAP bietet im Vergleich zu CEUS-LI-RADS signifikant bessere diagnostische Genauigkeit, Sensitivität und NPW mit unwesentlich geringerer Spezifität. Beide Algorithmen haben einen exzellenten PPW. Fusion der Kategorien LR-4 und LR-5 würde zu einer signifikanten Verbesserung der diagnostischen Genauigkeit von CEUS-LI-RADS für die Diagnose eines HCCs führen, die dann mit der von ESCULAP vergleichbar wäre.

\section{V13.002 Kontrastmittelsonografie (CEUS) bei Neugeborenen und Säuglingen: Single-Center-Erfahrungen}

Autoren Balzer AK ${ }^{1}$, Franke $D^{1}$

Institut 1 Medizinische Hochschule Hannover, Hannover/DE

DOI 10.1055/s-0037-1606925

Problemstellung Die Kontrastmittelsonografie wird in der Pädiatrie bisher nur in wenigen Zentren durchgeführt und ist in Europa weiterhin Off-Lable-Use. Wir stellen Indikationen, Ergebnisse und Sicherheitsaspekte bei Neugeborenen und Säuglingen vor.

Patienten und Methode Von März 2009 bis Mai 2017 wurden 331 Kinder mittels Kontrastmittelsonografie untersucht, darunter 52 Säuglinge (16\%) inklusiv drei Neugeborener. Ausgewertet wurden insgesamt $n=58$ Organuntersuchungen (davon bei sechs Kindern jeweils zwei Organe). Medianes Alter 5,5 Monate, Range 0 - 12 Monate. Das am häufigsten untersuchte Organ war die Leber mit $n=43$ (74\%), gefolgt von den Nieren und ableitenden Harnwegen mit $n=8(14 \%)$. Die Applikation des Kontrastmittels (SonoVue ${ }^{\circledR}$ ) erfolgte intravenös (96\%) oder intrakavitär (4\%). Follow-up Untersuchungen bei $n=9$ (17\%) Kindern.

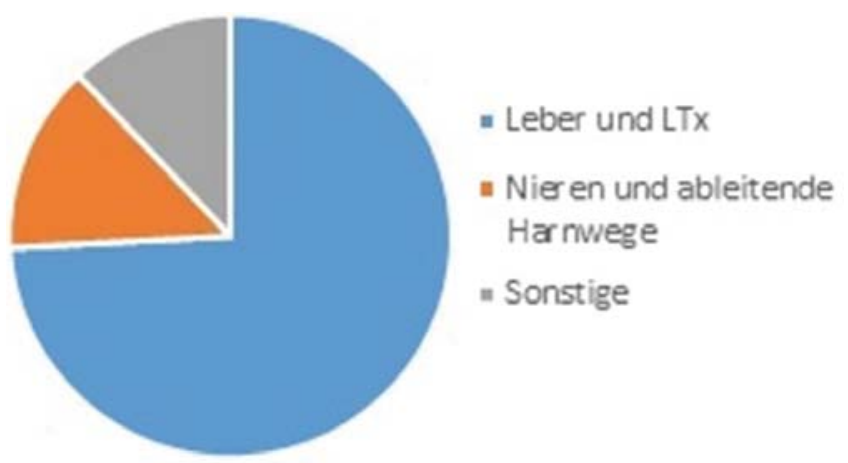

Abb. 1 Untersuchte Organe CEUS

Ergebnis Die häufigsten Indikationen waren fokale Leberläsionen (52\%), Perfusionsstörungen (16\%) und Verlaufskontrollen (9\%) der Leber. Maligne Leberläsionen fanden sich bei insgesamt $n=6$ (23\%) Kindern, darunter Neuroblastome (33\%), Hepatoblastome (15\%) und Metastasen (17\%). Bettseitige Untersuchungen auf der Intensivstation machten 33\% der Untersuchungen aus. Die intravenös applizierte Kontrastmitteldosis lag zwischen 0,2 und $2,5 \mathrm{ml}$. Bei keinem Kind traten Nebenwirkungen auf. Insgesamt waren $\mathrm{n}=52$ (90\%) der Untersuchungen von diagnostischem Wert.

Schlussfolgerung Die Kontrastmittelsonografie bei Säuglingen ist ein extrem hilfreiches und sicheres Verfahren, das bei schwerkranken oder immobilen
Kindern MRT und CT-Untersuchungen reduzieren oder ersetzen konnte und in der Mehrzahl der Fälle diagnostisch war.

\section{V13.003 Zur sonografischen Klassifikation der Leberhämangiome}

Autoren Kallenbach $\mathrm{M}^{1}$, Dörffel $\mathrm{Y}^{1}$, Wermke $\mathrm{W}^{1}$

Institut 1 Charité Universitätsmedizin Berlin, Berlin/DE

DOI 10.1055/s-0037-1606926

Problemstellung Hämangiome sind häufige Befunde bei Patienten mit malignen Erkrankungen. Die Kenntnis der unterschiedlichen Kontrastierungsverläufe ist zur Abgrenzung von bösartigen Leberläsionen hilfreich. Deshalb wurde von W. Wermke 2006 eine Einteilung der Hämangiome anhand des Kontrastverhaltens vorgenommen.

Patienten und Methode Über einen Zeitraum von 18 Monaten wurden 125 konsekutive Patienten mit 132 Leberläsionen, die ein für Hämangiome eindeutiges Kontrastierungsmuster aufwiesen, eingeschlossen. Mittels CEUS erfolgte eine detaillierte Beschreibung der auftretenden Kontrastierungsphänomene, sowie eine Einstufung in die von Wermke definierten 5 Typen.

Ergebnis 78/132 (59\%) kontrastierten sich allmählich - entweder vollständig ( $n=8 / 78,10 \%)$ oder teilweise $(n=70 / 78,90 \%)$ in zentripetaler Richtung. Die Hälfte der Tumorschnittfläche kontrastierte sich nach 108 s p.i. Eine Minderkontrastierung im Untersuchungsverlauf zeigten 13\% dieser Fälle.

54/132 (41\%) kontrastierten sich rasant, wobei bis zur 30. Sekunde p.i. mindestens 50\% der Tumorschnittfläche mit Mikroblasen gefüllt war. Im Mittel wurde dies $19 \mathrm{~s}$ p.i. beobachtet. Eine Minderkontrastierung im Untersuchungsverlauf wurde bei $28 \%$ dieser Hämangiome beobachtet.

Weitere Kontrastierungsphänomene fielen bei 16\% ( $n=21 / 132)$ der Hämangiome auf, davon zeigten $\mathrm{n}=3$ eine vorzeitige Lebervenenkontrastierung, $\mathrm{n}=15$ Pseudoläsionen durch arterioportale Fisteln und $\mathrm{n}=5$ beide Phänomene.
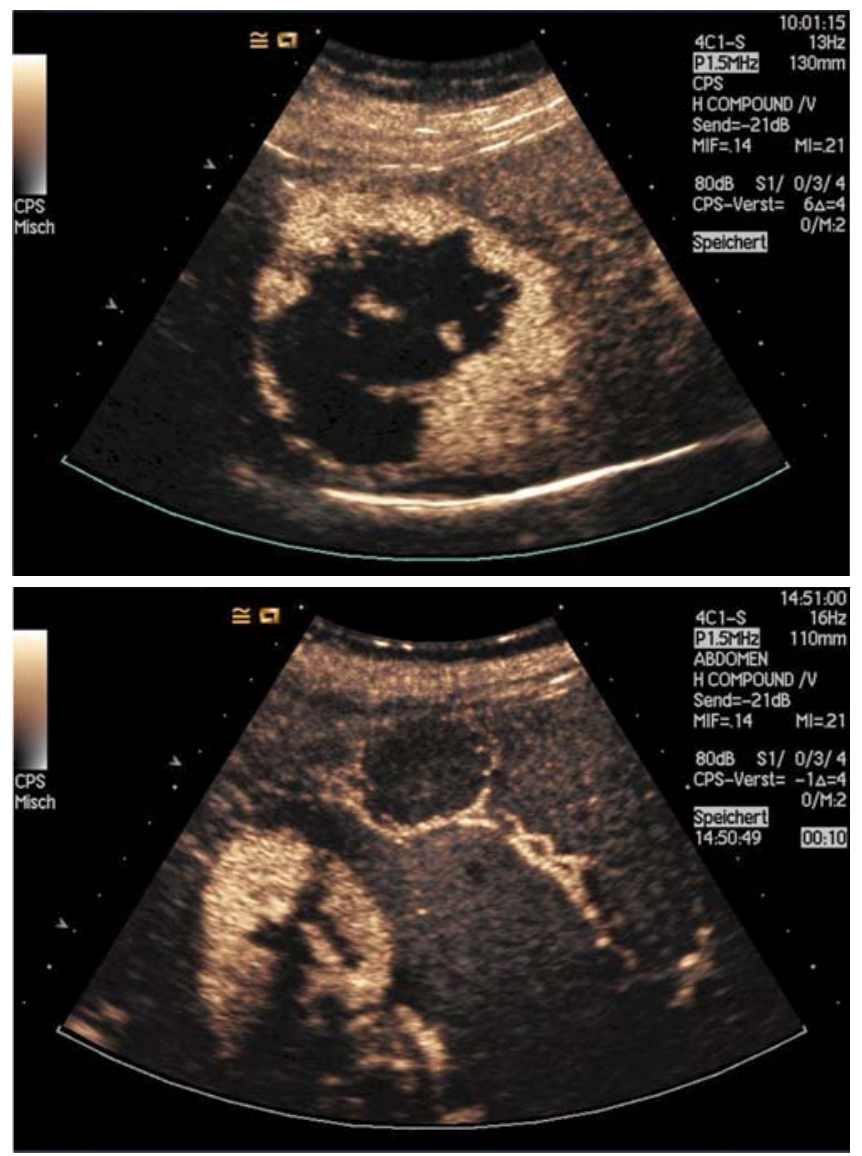
Schlussfolgerung Das unterschiedliche Kontrastierungsverhalten der Hämangiome lässt folgende Einteilung zu: Typ 1 - langsamer Blutfluss mit komplettem (1a) oder inkomplettem Irisblendenphänomen (1b), Typ 2 - hoher Blutfluss mit rascher Kontrastierung von 50\% der Schnittfläche innerhalb von 30 Sekunden. Ein Teil der Hämangiome des Typs 2 zeigen passagere Pseudoläsionen oder vorzeitige Venenkontrastierung. Diesen Phänomenen liegen arterioportale und arteriovenöse Fisteln zugrunde, welche eine weitere Differenzierung erlauben (Typ $3-5)$.

\section{8: Kopf/Hals}

\section{V8.001 Sonografische Charakteristika bei Schilddrüsenkarzinomen}

\section{Autoren Petz $\mathrm{V}^{1}$, Zettinig $\mathrm{G}^{1}$}

Institut 1 Schilddrüsenpraxis Josefstadt, Wien/AT

DOI 10.1055/s-0037-1606927

Problemstellung Während die Prävalenz von Schilddrüsenknoten in Österreich sehr hoch ist, ist die Inzidenz von Schilddrüsenkarzinomen relativ gering. Deshalb ist die präoperative Identifizierung von Knoten mit einer hohen Malignitätswahrscheinlichkeit entscheidend. Es wurden bereits Studien zu dem Thema ,sonografische Malignitätskriterien von Schilddrüsenknoten und Schemata für deren strukturierte Beurteilung، veröffentlicht. Bisher ist allerdings unbekannt wie weit diese Schemata auch im Jodmangelgebiet Österreich anwendbar sind.

Patienten und Methode Es wurden die sechs wichtigsten bisher beschriebenen sonografischen Malignitätskriterien von Schilddrüsenknoten mithilfe des Vergleichs von präoperativen Ultraschallbildern von 76 Karzinom- und 147 Struma-PatientInnen der Schilddrüsenpraxis Josefstadt, welche zwischen 2004 und 2014 prä- und postoperativ vorstellig gewesen waren, untersucht. In einer 'geblindeten' ExpertInnensitzung wurden die anonymisierten Ultraschallbilder beurteilt. Ziel dieser retrospektiven Studie war es, folgende sonografische Malignitätskriterien auf ihre Aussagekraft im Jodmangelgebiet Österreich zu testen: ,Hypoechogenität', ,deutliche Hypoechogenität', ,fehlender Halo', ,Mikrolobuli bzw. irregulärer Randsaum', ,Mikroverkalkungen' und ,taller than wide".

Ergebnis Alle bis auf das Merkmal ,Hypoechogenität‘ zeigten sich signifikant häufiger in malignen als in benignen Läsionen. Bei papillären Karzinomen traf dies für ,fehlender Halo' nicht mehr zu. Als wichtige Malignitätskriterien kristallisierten sich ,deutliche Hypoechogenität', ,Mikroverkalkungen', ,Mikrolobuli bzw. irregulärer Randsaum‘ sowie ,taller than wide“ heraus. Außerdem stieg die Merkmalsanzahl mit der Malignität, durchschnittlich zeigten Karzinome $(2,8 \pm 1,4$ bzw. PTC $3,0 \pm 1,3)$ mehr Merkmale als Kontrollen $(1,7 \pm 1,2)$. Schlussfolgerung Es konnte gezeigt werden, dass sich das Ultraschallmuster eines benignen Knotens von dem eines malignen Knotens unterscheidet und das Vorhandensein oder Fehlen von bestimmten Ultraschallkriterien im Jodmangelgebiet Österreich einen Hinweis auf die Malignität einer Läsion gibt.

\section{V8.002 Sonografie der Virchow-Drüse: Frühdiagnostik von Fernmetastasen am Venenwinkel bei abdominellen Tumoren}

Autoren Seeger $\mathrm{M}^{1}$

Institut 1 Unversitätsklinik Schleswig-Holstein, Campus Kiel DE, Kiel/DE DOI 10.1055/s-0037-1606928

Problemstellung Als Virchow-Drüse werden Fernmetastasen an der Einmündung des Ductus thoracicus am Venenwinkel bei abdominellen Tumoren bezeichnet, ihr Nachweis ist für die Bestimmung des Tumorstadiums und eine stadiengerechte Therapie wichtig. Die Palpation wird durch die Clavicula und den M. sternocleidomastoideus erschwert, in der klinischen Untersuchung werden häufig erst ausgedehnte Befunde entdeckt. Durch Sonografie mit
Linearsonden am Venenwinkel müsste sich die Diagnostik und Früherkennung von Metastasen am Venenwinkel deutlich verbessern lassen.

Patienten und Methode Bei 520 Patienten mit abdominellen Tumor wurde im Rahmen der Erstdiagnostik und des Stagings der Venenwinkel beidseits und der Ductus thoracicus mit Lineararsonden untersucht, die Befunde wurden mit den Palpationsbefunden verglichen. Nach der Untersuchung wurde erneut ein Tastbefund erhoben. Als suspekt wurden Lymphknoten mit aufgehobener Architektur, unregelmäßiger Begrenzung oder infiltrativem Wachstum gewertet. Auch eine Tumorbeteiligung des distalen Ductus thoracicus und der lokalen Venen wurde miterfasst.

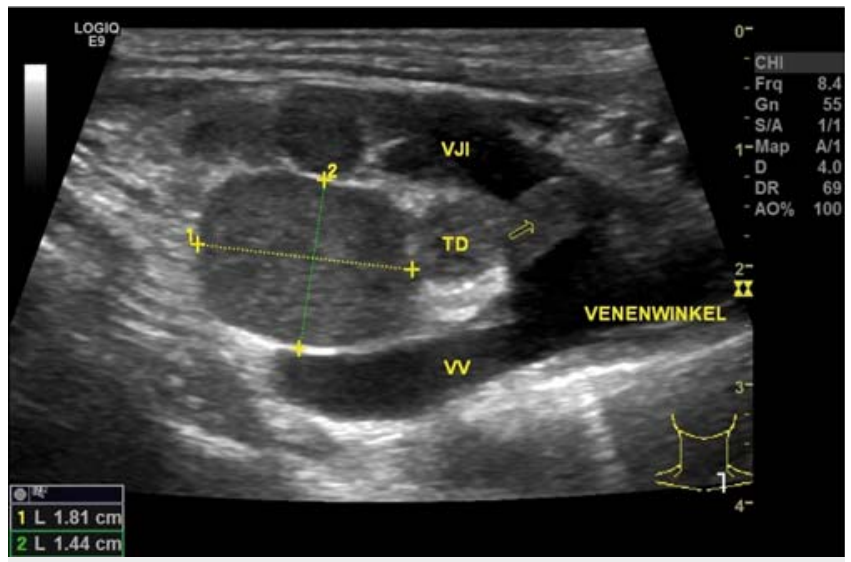

Abb. 1 Lymphknotenmetastase am linken Venenwinkel bei PankreasCA. Aus dem Ductus thoracicus (TD) ragt ein Tumorzapfen (Pfeil) in den Venenwinkel. Längsschnitt linker Venenwinkel, VJI: V. jugularis interna, VV: V. vertebralis.

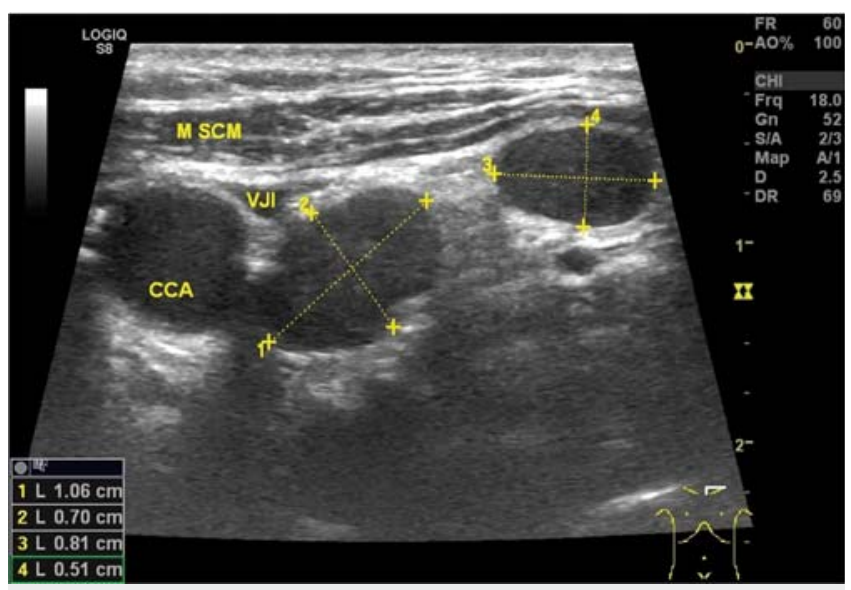

Abb. 2 Lymphknotenmetastasen am linken Venenwinkel bei neuroendokrinem Tumor des Dünndarms. Querschnitt durch den linken Venenwinkel, AO: Aorta, VJI: V. jugularis intern, M SCM: M. sternocleidomastoideus.

Ergebnis 32 von 520 Patienten mit abdominellen Tumoren hatten in der Sonografie metastasensuspekte Lymphknoten am Venenwinkel, 30 Patienten am linken Venenwinkel, zwei Patienten am rechten Venenwinkel. Bei 4 Patienten war ein Tumorwachstum im distalen Ductus thoracicus darstellbar. Nur bei 8 von diesen 32 Patienten (25\%) waren auffällige Lymphknoten in der klinischen Untersuchung getastet worden. In Kenntnis des Ultraschallbefundes konnten bei 18 von 32 Patienten Lymphknoten am Venenwinkel palpiert werden (56\%).

Schlussfolgerung Die Sonografie des Venenwinkels verbessert die Diagnostik von Lymphknotenmetastasen am Venenwinkel im Vergleich zur Palpation erheblich. Zusätzlich können weitergehende Aussagen über lokale Komplikationen und eine Tumorbeteiligung des Ductus thoracicus gemacht werden. Das 
Tumorstaging wird dadurch wesentlich verbessert, nicht stadiengerechte Behandlungen können vermieden werden.

\section{V8.003 Integration von dopplersonografisch untersuchter Perforator-Anatomie in die CAD/ CAM-Planung mikrovaskulärer Kieferrekonstruktion}

\author{
Autoren Goetze Ej ${ }^{1}$, Moergel $\mathrm{M}^{1}$, Al-Nawas $\mathrm{B}^{1}$ \\ Institut 1 Universitätsmedizin Mainz, Mainz/DE \\ DOI 10.1055/s-0037-1606929
}

Problemstellung Ultraschall ist ein etablierter Baustein in der präoperativen Diagnostik mikrovaskulärer Transplanate. Für die Rekonstruktion von Weich/ Hartgewebsdefekte übliche CAD/CAM-Planungen berücksichtigen diese Daten jedoch üblicherweise nicht. Wir präsentieren eine Methode zur Integration von dopplersonografisch detektierten Hautperforatoren in die 3D-Planung mikrovaskulärer Fibulatransplantate.

Patienten und Methode Nach Darstellung der Hautperforatoren des Fibulatransplantates und Dokumentation auf einer Messhilfe wurden diese in Bezug auf das obere Sprunggelenk dokumentiert. Diese Daten wurden in der Lokalisation auf die virtuelle Planung des Transplantates übertragen und in der weiteren 3D-Planung in der Ausrichtung miteinbezogen. Auf dieser Basis entstanden Schnittschablonen für Kiefer und Bein. Intraoperativ erfolgte eine dopplersonografische Gegenkontrolle der Perforatoranatomie.

Bei 35 Patienten wurde die Prozedur in 25 primären bzw. 10 sekundären Rekonstruktionen angewendet (Alter: $51 \pm 7$, w:m 1:1).

Ergebnis Planungslokalisation und klininsche Präsentation intraoperativ unterschieden sich in keinem Fall mehr als $5 \mathrm{~mm}$. Von chirurgischer Seite wurde die präoperative Planung wurde als hilfreich für Design der Hautinsel und Platzierung der Osteotomien eingeschätzt. Die Schnittschablonen fanden immer Verwendung.

Hautinseln wurden intraoral $(n=20)$, extraoral $(n=7)$ und geteilt $(n=3)$ angelegt. Es kam zum Verlust von 4 Transplantaten. Zusätzlich traten bei 6 Hautinseln ein partieller und einmal ein totaler Verlust auf. Diese Ergebnisse traten in Patienten mit Risikofaktoren wie präoperative Bestrahlung, fortgeschrittene Leberzirrhose o.ä. auf.

Schlussfolgerung Es ist möglich Hautperforatoren mittels dopplersonografischer Daten erfolgreich in eine 3D-Planung einzubinden. Eine weitere Integration von Weichgewebsaspekten ist noch nicht etabliert. Design und Splitting von Hautinseln sind ohne Präparation der Perforatoren möglich.

\section{V8.004 Veränderungen in der Sonografie der Speicheldrüsen bei Patienten mit Sjögren-Syndrom im Fünf-Jahres-Verlauf}

Autoren Hofauer $\mathrm{B}^{1}$, Mansour $\mathrm{N}^{2}$, Knopf $\mathrm{A}^{3}$

Institut 1 Klinikum rechts der Isar, München/DE; 2 Klinikum rechts der Isar der TU München, München/DE; 3 Klinikum rechts der Isar, TU München, München/DE DOI 10.1055/s-0037-1606930

Problemstellung Der Stellenwert sonografischer Verfahren in der Beurteilung der Speicheldrüsenbeteiligung des Sjögren-Syndroms (SjS) ist Bestandteil laufender Untersuchungen. Es wurden signifikante Unterschiede beim Einsatz von elastografischen Verfahren zwischen Patienten mit einem SjS und einer Sicca-Symptomatik beschrieben. Bisher liegen keine Untersuchungen zu longitudinalen Veränderungen der Speicheldrüsen bei Patienten mit einem SjS vor. Patienten und Methode Patienten mit einem primären SjS wurden in die Untersuchung eingeschlossen. Für die Evaluation der subjektiven Beschwerden wurde der ESSPRI verwendet und systemische Manifestationen anhand des ESSDAI dokumentiert. Zwischen 2011 und 2017 wurden die sono- und sonoelastografischen Veränderungen der Speicheldrüsen dokumentiert. Angewendet wurde die B-Mode-Sonografie, Realtime-Elastografie, Virtual Touch Imaging und Scherwellenelastografie.
Ergebnis Es wurden 50 Patienten eingeschlossen (45 weiblich, Alter: $56 \pm 16$ ). Zu Beginn betrug der ESSPRI 8,3 $\pm 4,6$ und der ESSDAI 5,6 $\pm 7,5$. Der mittels B-Mode-Sonografie erhobene Wert für die Gl. parotis betrug 1,6 $\pm 0,6$ und für die Gl. submandibularis 1,7 $\pm 1,0$. Der Wert der Scherwellenelastografie der $\mathrm{Gl}$. parotis $2,99 \mathrm{~m} / \mathrm{s} \pm 0,93$ und der $\mathrm{Gl}$. submandibularis $2,15 \mathrm{~m} / \mathrm{s} \pm 0,57$. Die Untersuchungen wurden nach fünf Jahren wiederholt. Hier zeigte sich eine Reduktion des ESSPRI auf $6,1 \pm 3,2(p=0,002)$ und des ESSDAI auf 4,6 $\pm 7,0$ $(p<0,001)$. Die B-Mode-Sonografie ergab einen Wert von $1,4 \pm 0,7$ $(p<0,001)$ für die Gl. parotis und $1,9 \pm 1,0(p=0,034)$ für die Gl. submandibularis. Es zeigte sich eine Reduktion der Scherwellenelastografie der Gl. parotis auf $2,33 \mathrm{~m} / \mathrm{s} \pm 0,7(\mathrm{p}<0,001)$. Die übrigen Methoden ergaben keine Veränderungen.

Schlussfolgerung In der Fünf-Jahres-Verlaufskontrolle zeigten sich unabhängig von der Erkrankungsdauer lediglich dezente Veränderungen der B-Modesonografischen Ausprägung des SjS. Es zeigte sich ein deutlicher Rückgang der Werte der Scherwellenelastografie für die Gl. parotis, was auf eine gewisse Modulierbarkeit in diesem Bereich hinweisen könnte.

\section{V8.005 Speicheldrüsensonografie in der Differentialdiagnostik von Patienten mit Siccasymptomatik}

Autoren Bergner $\mathrm{R}^{1}$, Wadsack $\mathrm{D}^{1}$, Sattler $\mathrm{H}^{1}$, Löffler $\mathrm{C}^{2}$

Institut 1 Klinikum Ludwigshafen, Ludwigshafen/DE; 2 Universitätsklinikum Mannheim, Mannheim/DE

DOI 10.1055/s-0037-1606931

Problemstellung Studien konnten zeigen, dass Patienten mit primärem Sjögren-Syndrom (pSS) verglichen mit Gesunden typische sonografische Veränderungen aufwiesen. Es ist allerdings unklar, ob diese Veränderungen spezifisch sind für das pSS verglichen mit anderen Kollagenosen mit Ro-SSA-AK oder Siccasymptomatik.

Patienten und Methode Wir verwendeten einen von Zhang et al. entwickelten Ultraschallscore (USGs) um Speicheldrüsen zu untersuchen. Hierbei konnte ein Score von 0-maximal 48 erreicht werden. Wir verglichen dabei 6 verschiedene Gruppen. Patienten mit: (Gruppe 1) einem pSS; (Gruppe 2) einem pSS aber Ro-SSA/La-SSB-AK negativ; (Gruppe 3) sekundärem Sjögen-Syndrom (sSS); (Gruppe 4) anderen rheumatischen Erkrankungen (ORD) ohne Ro-SSAAK; (Gruppe 5) ORD ohne Siccasymptome aber positiven Ro-SSA-AK; (Gruppe 6) Kontrollgruppe.

Soweit verfügbar wurde der USGs mit der Speicheldrüsenszintigrafie korreliert.

Ergebnis Wir schlossen 121 Patienten in die Untersuchung ein. Der USGs lag bei 16,7 $\pm 10,9$ (Gruppe 1); 2,6 $\pm 3,7$ (Gruppe 2); 9,25 $\pm 9,9$ (Gruppe 3); 4,8 $\pm 6,4$ (Gruppe 4); 4,5 $\pm 6,7$ (Gruppe 5) und 1,7 $\pm 2,1$ (Gruppe 6). Patienten mit pSS hatten einen signifikant höheren Score wie alle anderen Gruppen $(p<0,0001)$. Patienten mit sSS hatten signifikant höheren Score wie die Kontrollgruppe $(p<0,008)$. Die übrigen Gruppen unterschieden sich nicht signifikant untereinander.

Bei 48 Patienten waren Speicheldrüsenszintigrafien verfügbar. Die szintigrafische Speicheldrüsenfunktion korrelierte signifikant mit dem USG $(r=0,62 p<0,00001)$.

Schlussfolgerung USG zeigen beim pSS signifikant höhere Scores, wie Patienten mit ORD, unabhängig davon, ob diese Ro-SSA-AK und/oder eine Siccasymptomatik aufwiesen. USG als nicht invasives Verfahren ist daher ein hilfreiches Diagnostikum in der Abgrenzung des pSS. 


\section{V8.006 Benefit von B-Mode, FKDS und CEUS bei Paragangliomen des Glomus caroticum}

Autoren Mansour $\mathrm{N}^{1}$, Hofauer $\mathrm{B}^{1}$, Knopf $\mathrm{A}^{1}$

Institut 1 Klinikum rechts der Isar der TU München, München/DE

DOI 10.1055/s-0037-1606932

Problemstellung Paragangliome des Glomus caroticum (PGC) sind seltene Tumoren, die von sympathischen Fasern im Bereich der Karotisbifurkation ausgehen, stark vaskularisiert sind und sich durch eine schmerzlose, langsam progrediente Schwellung hochzervikal bemerkbar machen. Die Therapie der Wahl ist die chirurgische Resektion, wobei Tumoren mit Gefäßummauerung mit der Option eines Gefäßersatzes operiert werden müssen. Diese Patienten gilt es, präoperativ zu identifizieren.

Patienten und Methode Von 2009 bis 2016 wurden 9 Patienten mit 11 PGC bei uns mittels B-Mode US, farbkodierter Duplexsonografie (FKDS) und CEUS untersucht. Die Diagnose wurde anschließend mittels DOTATOC-PET/CT bestätigt.

Ergebnis Von den neun Patienten zeigten zwei einen beidseitigen Befund. B-Mode US, FKDS und CEUS zeigten bei einem der elf Tumoren eine Ummauerung der A. carotis interna, so dass dieser durch die Gefäßchirurgen operiert wurde.

Schlussfolgerung Mittels B-Mode US, FKDS und CEUS ist es dem Kopf-HalsChirurgen möglich, eine Ummauerung der Gefäße zu beurteilen. Zusätzlich bietet der CEUS eine nebenwirkungsarme Option, die Effektivität einer präoperativ durchgeführten Embolisation zu überprüfen.

\section{V8.007 Kontrast-verstärkte Sonografie zur Differenzierung echoleerer Kopf-Hals-Läsionen}

Autoren Knopf $A^{1}$

Institut 1 Klinikum rechts der Isar, TU München, München/DE DOI 10.1055/s-0037-1606933

Problemstellung Die Kopf-Hals-Region ist ein Schmelztiegel an Erkrankungen des dysontogenetischen, entzündlichen und neoplastischen Formenkreises. Die Sonografie kommt aufgrund ihrer hohen Ortsauflösung flächendeckend zum Einsatz. Insbesondere echoleere Läsionen, die keine eindeutige Makrovaskularisation in der FKDS aufweisen, erschweren jedoch die eindeutige Zuordnung der Entität und damit ein zielgerichtetes therapeutisches Vorgehen. Der Stellenwert kontrast-verstärkter Sonografie zur Beurteilung der Perfusion in der Differenzierung echoleerer Läsionen ist bislang unklar.

Patienten und Methode Es wurden 36 Patienten mit Kopf-Hals-Läsionen eingeschlossen, die nach HR-B-Bild Sonografie (9Mhz, linear, THI, Siemens S2000) und FKDS nicht weiter differenziert werden konnten. Patientenbezogene Daten (Alter, Geschlecht, Noxenstatus) sowie Ergebnisse der HNO-Spiegeluntersuchung wurden retrospektiv erfasst. Zur weiteren läsionalen Differenzierung wurden fraktioniert 2,5 ml SonoVue appliziert und das Perfusionsverhalten qualitativ und quantitativ (Bracco VueBox) erfasst.

Ergebnis Die vorliegende Studie schloss fünf exentrische Parotistumore, acht venöse Malformationen, fünf mediane und 10 laterale Halszysten, ein papilläres Schilddrüsenkarzinom ex medianer Halszyste, fünf zystisch regressive Metastasen und zwei Lymphozelen ein. Die Sensitivität und Spezifität der HR-BBild Sonografie und FKDS betrug 53\% bzw. 97\% und konnte durch Einsatz kontrastverstärkter Sonografie auf $92 \%$ und 99\% verbessert werden.

Schlussfolgerung Die kontrast-verstärkter Sonografie stellt eine sinnvolle Ergänzung der HR-B-Bild Sonografie und FKDS insbesondere bei echoleeren Läsionen dar.

\section{F 4 Tularämie - Fallbericht über einen protrahierten Verlauf}

Autoren Gass $M^{1}$, Schmelzeisen $R^{1}$, Semper-Hogg $W^{1}$, Füssinger $M A^{1}$ Institut 1 Universitätsklinikum Freiburg, Freiburg/DE DOI 10.1055/s-0037-1606934

Problemstellung Die Tularämie ist eine seltene, jedoch hochinfektöse Erkrankung bei der unbehandelt eine Letalität von über 30\% beschrieben ist. Die unspezifische Symptomatik und die niedrige Inzidenz erschweren die Diagnosestellung. Im vorliegenden Fallbeispiel wird die Diagnosestellung durch die Sonografie, die CT Diagnostik und der PCR ermöglicht.

Patienten und Methode Frau M.S., 66-jährig stellte sich bei uns mit einer Verschlechterung des Allgemeinzustandes, einer kürzlich aufgetretenen Lymphadenopathie, einer intraoralen ulzerierenden Läsion und einer B-Symptomatik vor. Nach einer Probeexzision des intraoralen Befundes wurde die Patientin zur intravenösen Antibiose aufgenommen. Der sonografische Befund zeigte multiple echoleere, rundliche Raumforderungen mit vermehrter randständiger Perfusion und dorsaler Schallverstärkung mit V.a. Lymphknoteneinschmelzung. Nach einer Verschlechterung des klinischen Bildes und einer Größenzunahme der Raumforderungen in der Sonografie, entschieden wir uns zur Anfertigung eines CT und zur Inzision der abszedierten Raumforderungen. Das histopathologische Ergebnis der ersten Probeexzision erwies sich als nekrotischer Zelldetritus mit granulozytärer Demarkation. Die im Rahmen der Inzision entnommenen Proben führten molekularbiologisch mittels PCR zur Diagnose einer Tularämie, woraufhin die Antibiose umgestellt wurde und sich klinisch wie auch sonografisch eine deutliche Regredienz zeigte.

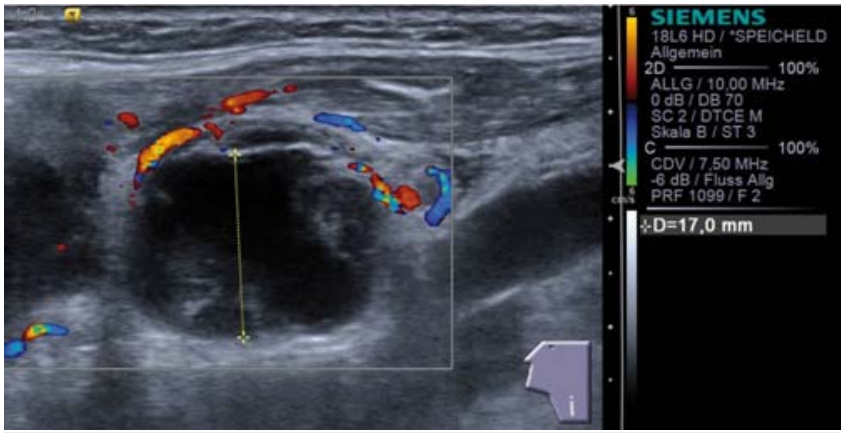

Abb. 1 Eingeschmolzener Lymphknoten

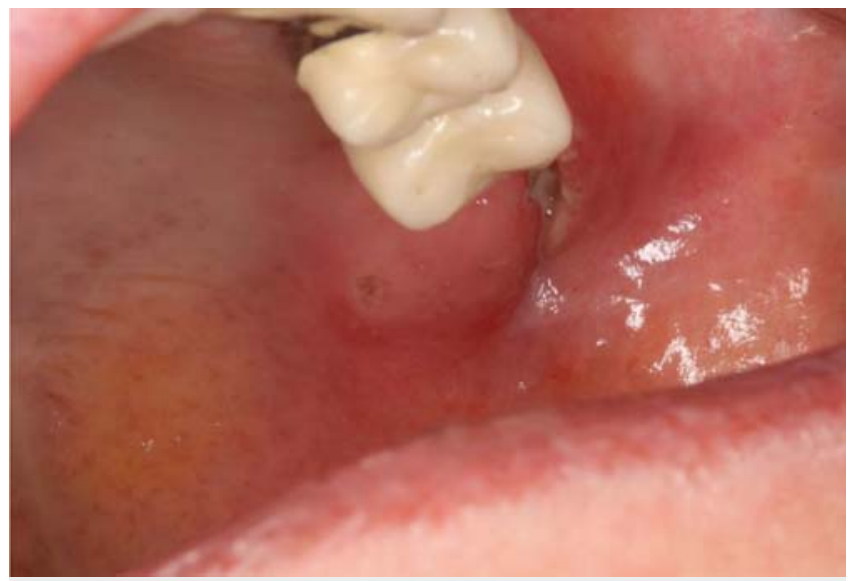

Abb. 2 Ulzerierende Läsion retromolar 


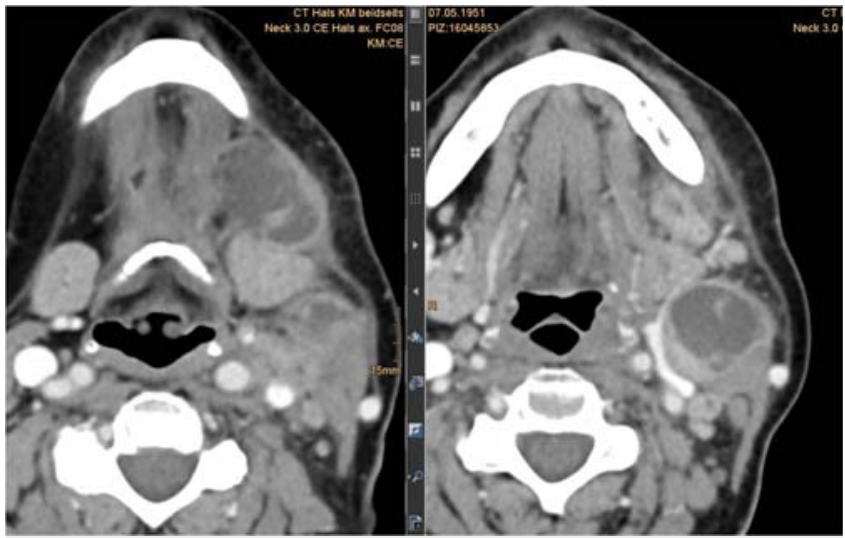

Abb. 3 CT mit KM

Ergebnis Die ulzeroglanduläre Tularämie charakterisiert sich durch ulzerierende Läsionen an der Eintrittsstelle mit regionaler, oft eitriger Entzündung der Lymphknoten. Die zielführende Diagnostik konnte bei der Patientin nicht durch eine histopathologische Bewertung erfolgen, sondern letztendlich durch eine mikrobiologische Untersuchung, sowie die klinische und sonografische Entwicklung gesichert werden.

Schlussfolgerung Unspezifische Raumforderungen im Halsbereich sind sonografisch bezüglich Ihrer Entität schwer zu beurteilen. Der vorliegende Fall zeigt, dass die Sonografie einen essentiellen Beitrag zur vollständigen Diagnostik bei unklaren Lymphadenopathien darstellt.

\section{V8.008 Sonografie der fokalen Schilddrüsenläsionen mit FDG-Anreicherung im PET-CT}

\section{Autoren Fernandez Sanchez J ${ }^{1}$ \\ Institut 1 Robert-Bosch-Krankenhaus Stuttgart, Stuttgart/DE \\ DOI 10.1055/s-0037-1606935}

Problemstellung Ein PET-CT wird vorwiegend bei onkologischen Fragestellungen, insbesondere bei Bronchialkarzinomen durchgeführt. Als Zufallsbefund werden manchmal fokale Läsionen in der Schilddrüse (SD) mit FDG-Anreicherung festgestellt. Ziel dieser prospektiven Studie ist die sonografische Evaluation dieser SD-Läsionen und ihrer Katalogisierung mittels TIRADS.

Patienten und Methode Bei 42 Patienten mit einer sog. PET-positiven fokalen SD-Läsion im PET-CT wurde eine weitere Abklärung mittels konventioneller Sonografie, Color Doppler und Elastosonografie durchgeführt. Danach erfolgte eine histologische Klärung und/oder eine Feinnadelpunktion und/oder eine sonografische Verlaufskontrolle (6 bis 18 Monate).

Ergebnis Bei 5/42 Patienten handelte es sich um TIRADS 4b bis 5 Läsionen. Hier wurde in allen Fällen ein SD-Karzinom festgestellt. 2/4 TIRADS 4a-Läsionen waren maligne. In keiner der TIRADS 3 Läsionen $(n=31)$ und TIRADS 2-Läsionen $(n=2)$ wurde histologisch oder zytologisch eine Malignität diagnostiziert.

Schlussfolgerung Eine fokale SD-Läsion mit FDG-Anreicherung in PET-CT sollte stets zuerst sonografisch weiter aufgeklärt werden. Diese Läsionen entsprechen in den meisten Fällen TIRADS-3-Knoten. Bei PET-positiven fokalen SDLäsionen, die sonografisch als TIRADS $4 \mathrm{~b}$ bis 5 klassifiziert sind, sollte immer eine histologische Klärung erfolgen.

\section{10: MSK/Nervensonografie}

\section{V10.001 Ultraschall gesteuerte Biopsie von osteolytischen Knochenläsionen}

\author{
Autoren Nitsch $T^{1}$, Schicht $C^{2}$, Südhoff $T^{1}$ \\ Institut 1 Klinikum Passau, Passau/DE; 2 Klinik am Goldenen Steig, \\ Waldkirchen/DE \\ DOI 10.1055/s-0037-1606936
}

Problemstellung Knochenmetastasen treten bei Krebspatienten häufig auf. Es gibt verschiedene Wege eine verlässliche Histologie zu gewinnen, wie z. B. die CT gesteuerte Punktion oder chirurgische Eingriffe. Die Sonografie ist eine einfache und häufig verfügbare Methode mit der auch eine Biopsie erfolgen kann. Jedoch ist Knochen nicht die Domäne der Sonografie, da mittels Sonografie die Knochenstruktur nicht dargestellt werden kann. Wird jedoch durch eine osteolytische Läsion die Knochenoberfläche zerstört, ergibt sich ein Fenster für Sonografie und entsprechend Biopsie. Wir berichten hier über die Effektivität und Komplikationsrate dieser Methode.

Patienten und Methode Dieses ist eine retrospektive Analyse der Ultraschallgesteuerten Biopsien von Osteolysen in unserer Abteilung. Die Biopsien wurden mit einem Thoshiba 400 Ultraschallgerät durchgeführt. Je nach Lokalisation erfolgte die Punktion mit einem „convex-array“ Punktionsschallkopf oder einem Linearschallkopf in „Freihand“-Technik. Wir benutzten True-cut Nadeln (Achieve) mit der Größe von 11 - 16G. Die Biopsien wurden unter Lokalanästhesie und aseptischen Bedingungen durchgeführt. Komplikationen wurden in drei Grade eingeteilt.

Ergebnis Wir führten 38 Biopsien mit folgenden Lokalisationen durch: Becken: 12, Rippen: 13, Brustbein: 3, Schienbein: 3, Wirbel: 2, Schädel: 1, Schlüsselbein: 1, Oberarm: 1, Schulterblatt: 1, Oberschenkel: 1. In 95\% erhielten wir eine verlässliche Histologie, die keine weitere Intervention erforderte. In diesen Fällen hatte die kleinste Läsion einen Durchmesser von $1 \mathrm{~cm}$. Lediglich in einem Fall (3\%) trat eine moderate Komplikation auf (selbstlimitierter Pneumothorax).

Schlussfolgerung Obwohl die Untersuchung des Knochens nicht die Domäne der Sonografie ist, zeigt unsere Analyse, dass die Ultraschall-gesteuerte Biopsie bei Osteolysen eine effektive und sichere Methode ist, eine verlässliche Histologie mit einer Rate von 95\% zu gewinnen.

\section{V10.002 Differenzierte Vermessung der Beckenneigung mit navigierten Ultraschall zur Luxationsvermeidung von künstlichen Hüftgelenken}

Autoren Kozak J ${ }^{1}$, Alk $A^{2}$, Martin $T^{1}$

Institut 1 Aesculap AG, Tuttlingen/DE; 2 Aesculap, Tuttlingen/DE

DOI 10.1055/s-0037-1606937

Problemstellung Die präoperative Analyse und die hierauf basierende Operationsplanung sind in der Orthopädie und Unfallchirurgie von zentraler Bedeutung, um ein bestmögliches Behandlungsergebnis zu erzielen. Individuelle anatomische Faktoren müssen erfasst werden, um hieraus die Operationsindikation abzuleiten, das Therapieziel zu definieren und ggf. eine personalisierte Operationsmethode ableiten zu können.

Patienten und Methode Die sonografische Bestimmung individueller anatomischer Faktoren, wie sie in der vorliegenden Arbeit anhand des Pelvic Tilt dargelegt wird, erfüllt die oben genannten Faktoren in vielerlei Hinsicht. Darüber hinaus handelt es sich hierbei um eine Methode, die für den Patienten keinerlei spezifische Risiken beinhaltet. Das generelle Potential der Ultraschall navigierten Vermessung anatomischer Strukturen (Beinachse, Pelvic Tilt) in der Orthopädie und Unfallchirurgie ist daher als sehr groß zu betrachten. Die vorliegende Arbeit legt dies anhand der komplexen Anatomie des knöchernen Beckens dar.

Ergebnis Insbesondere ist die Möglichkeit gegeben, dass der Tablet-PC ermittelt, ob die errechnete Inklination und die errechnete Anteversion außerhalb 
des vorgegebenen Winkelbereiches für die Inklination bzw. des vorgegebenen Winkelbereiches für die Anteversion liegen, wobei die Winkelbereiche diesmal auf die Frontalebene bezogen aufzufassen sind. Dadurch ist für den Operateur ein Hinweis anzeigbar, dass die Hüftpfanne am Becken so implantiert wird, dass sie außerhalb der „Safe Zone“ implantiert ist.
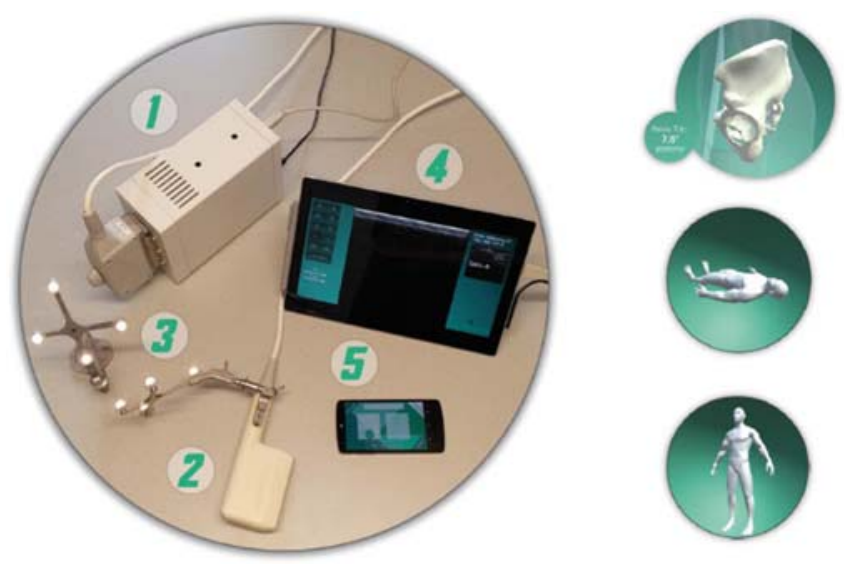

Abb. 1 Komponenten des Smart-Ultraschall-Systems

Schlussfolgerung Der Tablet-PC kann ferner ausrechnen, unter welchem Inklinationswinkel und welchem Anteversionswinkel die Hüftpfanne in Bezug auf die Beckeneingangsebene bei vorgegebenem Beckenneigungswinkel zu implantieren ist, damit die Hüftpfanne unter Berücksichtigung der Beckenneigung im Bereich der „Safe Zone“ implantiert wird.

\section{V10.003 Hüftluxation des Säuglings, Therapiemonitoring mittels transinguinalen Ultraschalls}

Autoren Zierl $\mathrm{A}^{1}$, Eberhardt $\mathrm{O}^{1}$, Nasko $\mathrm{M}^{1}$, Wirth $\mathrm{T}^{1}$, Kalle T von Institut 1 Olgahospital, Stuttgart/DE

DOI 10.1055/s-0037-1606938

Problemstellung Bei der Behandlung der Hüftdysplasie und -luxation erfolgt die Repositionskontrolle überwiegend mittels Röntgenbeckenübersichtsaufnahme, MRT oder CT. Der transinguinale Ultraschall, von van Douveren et al. 2003 etabliert und von Eberhardt et al. 2009 im Vergleich mit Arthrografie bzw. Röntgen validiert, ist als strahlungsfreies Real-Time-Verfahren eine exzellente Alternative.

Patienten und Methode Beim transinguinalen Ultraschall (Microkonvex-Sonde) in Hüftflexion und -abduktion bilden die Knochenkonturen des oberen Schambeinastes und des Schenkelhalses normalerweise eine modifizierte Ménard-Shenton-Linie, bei Dezentrierung zeigt sich eine Stufe. Neben knorpeligem Hüftkopf und Pfannendach sind evtl. repositionsverhindernde Interponate direkt sichtbar. Vor Gipsanlage wird die optimale Position für tiefe Zentrierung ermittelt. Mögliche Probleme können durch zusätzliche laterale (coronale) Darstellung (lineare Sonde) sowie topografisch orientierte Untersuchungstechnik vermieden werden.

Ergebnis Indikation und eigenes Vorgehen:

Die Hüftsprechstunde wird gemeinsam von Orthopädie, Kinderradiologie und Orthopädietechnik bestritten. Bei schwerer Dysplasie mit erforderlicher Retention wird mittels transinguinalen Ultraschalls die Stabilität auch unter Provokation überprüft und nach einer Woche erneut kontrolliert. Bei Instabilität wird auf die Gipsbehandlung gewechselt.

Gipsanlage und Gipswechsel erfolgen in Narkose im OP. Der Orthopäde positioniert das Hüftgelenk, vor Gipsanlage erfolgt der Ultraschall durch den Kinderradiologen von lateral linear und von transinguinal, die optimale Reposi- tionsposition wird ermittelt, Interponate werden direkt visualisiert. Nach Gipsanlage wird die Reposition transinguinal dokumentiert.

Bei arthroskopischer und offener Reposition wird der Schallkopf steril vom Operateur geführt, der Kinderradiologe bedient das Sonogerät, nach Gipsanlage Sonokontrolle im OP, am selben Tag MRT ohne Narkose.

Schlussfolgerung Intraoperative Repositionskontrollen, Therapieentscheidung und Therapiemonitoring bei Retentionsschienenbehandlung sind mittels transinguinalen Ultraschalls routinemäßig durchführbar. Voraussetzung ist eine gute Infrastruktur mit interdisziplinärer Zusammenarbeit.

\section{V10.004 Die Bedeutung der Strain-Elastografie für die Diagnose unspezifischer Rückenschmerzen}

Autoren Bauermeister $\mathrm{W}^{1}$, Raßmann $\mathrm{P}^{2}$

Institut 1 Schmerzinstitut, München/DE; 2 Schmerzinstitut München,

München/DE

DOI 10.1055/s-0037-1606939

Problemstellung Chronische Rückenschmerzen lassen mit herkömmlichen bildgebenden Verfahren in $85 \%$ der Fälle keine spezifische Ursache erkennen. Rückenschmerzen können durch eine neurogene Entzündung der Myofaszie entstehen, die dadurch verhärtet. Diese lässt sich mit der Indentometrie messen, jedoch erkennt man daraus nicht welche Gewebeschichten betroffen sind. Die Strain-Elastografie ermöglicht eine Abbildung der Gewebehärten, mit der Möglichkeit daraus eine kausale Behandlungsstrategie zu entwickeln. Hypothese.

Bei einer Härtedifferenz von $\geq 5 \%$ im Pixelcount der Strain-Elastografie im Seitenvergleich ist auf der härteren im Vergleich zur weicheren Seite mit der Indentometrie eine höhere Kraft von $\geq 10$ Newton erforderlich.

Patienten und Methode 30 Patienten mit einseitigen unspezifischen Rückenschmerzen wurden bilateral mit der Strain-Elastografie (Ultrasonix Tablet Analogic USA) am Rücken, Hüfte und Beinen untersucht. Die Indentometrie wurde mit dem Indentometer Pro (TU Chemnitz) durchgeführt. Mit der Software Image] wurden die Pixelanalyse der Elastografiebefunde durchgeführt. Eine sequentielle statistische Analyse erfolgte mit Bross Sequential Clinical Plan, Irrtumswahrscheinlichkeit $\alpha=5 \%$.

Ergebnis In der sequentiellen statistischen Analyse zeigte sich bereits nach 11 Probanden ein signifikantes Ergebnis mit Annahme der Nullhypothese. Auf der härteren Körperseite ist mit der Indentometrie eine signifikant höhere Kraft zum Erreichen einer definierten Eindringtiefe erforderlich.

Schlussfolgerung Die Annahme, dass bei chronischen Rückenschmerzen in $90 \%$ der Fälle keine spezifische Pathologie nachweisbar ist kann so nicht aufrechterhalten werden. In der Strain-Elastografie zeigen sich spezifische Verhärtungen in den Faszien und Muskeln die Folgen einer neurogenen Entzündung darstellen können. Mittels der Strain-Elastografie eröffnet sich eine neue Dimension der Diagnostik chronischer Rückenschmerzen.

\section{F 9 Rolle des Powerdopplers bei der klinischen Entscheidung zum akuten Ellenbogengelenk}

Autoren Maier $\mathrm{A}^{1}$, Scheel $\mathrm{M}^{1}$, Tiefenbach $\mathrm{M}^{1}$, Gehlen $\mathrm{M}^{2}$, Hammer $\mathrm{M}^{1}$ Institut 1 Nordwestdeutsches Rheumazentrum, St. Josef-Stift Sendenhorst, Sendenhorst/DE; 2 Der FÜRSTENHOF, Bad Pyrmont/DE

DOI 10.1055/s-0037-1606940

Patienten und Methode Plötzliche Steigerung der Entzündungsaktivität in einem isolierten Gelenk bei sonst stabiler rheumatoider Arthritis ist eine differenzialdiagnostische Herausforderung. Es kann sich um einen Schub der Grunderkrankung oder eine septische Arthritis handeln. Ferner kommen Kristallarthropathien in Frage.

50-jährige Patientin mit einer rheumatoiden Arthritis stellt sich mit einer akuten Schwellung und Bewegungseinschränkung des rechten Ellenbogens vor. Als Begleitsymptome wurden Schüttelfrost und Erbrechen berichtet. Die Grunderkrankung war mäßig gut kontrolliert: Die Prednisolon-Dosis betrug $7,5 \mathrm{mg} / \mathrm{Tag}$, als DMARD (disease-modifying anti-rheumatic drug) wurde Leflu- 
nomid $20 \mathrm{mg} /$ Tag eingenommen. Vor einer Woche kam es zu einer Gastroenteritis. Eine umgehend durchgeführte Punktatanalyse des betroffenen Gelenks zeigte eine Zellzahl von 58 Tausend/ $\mu$ l. Nach einer initial gering erhöhten systemischen Entzündungsaktivität kam es am Folgetag zu einem raschen Anstieg. Sonografisch zeigte sich eine Synovialitis Grad 3 des Gelenks, die in den täglichen Kontrollen unverändert erschien. Die Powerdoppler-Aktivität dagegen bildete sich vom Grad 3 am ersten Tag bis zum fehlenden Nachweis am dritten Tag zurück. Nachdem die mikrobiologische Diagnostik steril blieb, wurde eine intraartikuläre Injektion mit Triamcinolon-Hexacetonid durchgeführt.

Schlussfolgerung Die exakte Klärung der Genese einer plötzlichen Entzündungsaktivität in einem isolierten Gelenk muss erzwungen werden, um eine adäquate Therapie nicht zu verzögern. Eine erhöhte Aktivität im Rahmen der Grunderkrankung lässt sich mittels einer lokalen Steroid-Injektion limitieren. Das gleiche gilt für Kristallarthropathien. Eine septische Arthritis dagegen kann durch die genannte Intervention potenziert werden. Sie geht mit einer gesteigerten Mortalität einher und bedarf einer umgehenden antibiotischen Gabe und/oder eines operativen Eingriffs. Einer sonografischen Untersuchung mithilfe des Powerdopplers kommt in der Differenzialdiagnostik eine wichtige Rolle zu.

\section{V10.005 Nanochirurgie: Die sonografisch gesteuerte perkutane Durchtrennung des Retinaculum flexorum beim Karpaltunnelsyndrom}

Autoren Forster $\mathrm{B}^{1}$, Forster $\mathrm{I}^{2}$

Institut 1 Praxis, Winterthur/CH; 2 Kinderklinik, Zürich/CH

DOI 10.1055/s-0037-1606941

Problemstellung Von diversen Autoren wurden seit 2009 verschiedene Methoden publiziert, um das Retinaculum flexorum beim Karpaltunnelsyndrom unter Ultraschallkontrolle nanochirurgisch zu durchtrennen.

Zwei zentrale Probleme wurden in diesen Arbeiten nicht ausreichend gewürdigt.

1. Schutz des Nervus medianus vor Läsionen.

2. Schaffung eines sonografisch definierten Raums von welchem aus die Durchtrennung des Retinakulums vorgenommen werden kann.

Patienten und Methode Zur Lösung dieser Probleme haben wir bei 20 nicht selektionierten nanochirurgischen Retinakulumspaltungen folgende Schritte durchgeführt:

1. Einbringen des Lokalanästhetikums ( $2 \mathrm{ml}$ Lidocain $1 \%$ mit Epinephrin) unter sonografischer Kontrolle ausschließlich zwischen dem Retinaculum flexorum und der Subkutis.

2. Einbringen von $0.1 \mathrm{ml}$ Hyaluronsäure durch Hydrodissektion zwischen dem Retinaculum flexorum und dem subkutanen Fettgewebe.

Ergebnis

1. Wir haben in allen 20 Fällen eine ausreichende Anästhesie erreicht um das Retinaculum flexorum mit dem Mikromesser zu durchtrennen. Sämtliche Patienten behielten während des gesamten Eingriffes die volle Sensibilität des Nervus medianus.

2. Durch das Einbringen von $0.1 \mathrm{ml}$ Hyaluronsäure durch Hydrodissektion zwischen dem Retinaculum flexorum und dem subkutanen Fettgewebe konnten wir einen sonografisch freien Raum erzeugen. Bei keinem der Patienten trat postoperativ eine Reaktion auf diese Substanz ein.

Schlussfolgerung Diese beiden Massnahmen erhöhen die Sicherheit und Reproduzierbarkeit bei der nanochirurgischen Durchtrennung des Retinaculum flexorum.

\section{V10.006 Nachweis von Neurogenen Entzündungen beim Myofaszialen Schmerzsyndrom mittels Strain-Elastografie und Validierung durch Algometrie}

Autoren Bauermeister $\mathrm{W}^{1}$, Raßmann $\mathrm{P}^{2}$

Institut 1 Schmerzinstitut, München/DE; 2 Schmerzinstitut München, München/DE

DOI 10.1055/s-0037-1606942

Problemstellung Myofasziale Schmerzsyndrome werden verursacht durch Trigger-Punkte oder Trigger-Regionen der Faszien und Muskeln die eine neurogene Entzündung in sich bergen. In den Faszien kann sich das als Verhärtungen mit Fibrosierung, in den Muskeln als Sarkomerkontrakturen mit knotenförmigen Verhärtungen äußern. Die neurogene Entzündung in den TriggerRegionen lässt sich durch eine Erniedrigung der Schmerzdruckschwelle, gemessen mit einem Algometer, objektivieren. Jedoch erkennt man daraus nicht, in welchen Gewebeschichten verhärtete Trigger-Areale liegen. Die Strain-Elastografie ermöglicht eine Differenzierung der Gewebeschichten, mit der Möglichkeit einer Lokalisierung und gezielten Behandlung der Trigger-Areale.

Hypothese Bei einer Härtedifferenz von $\geq 5 \%$ im Pixelcount der Strain-Elastografie im Seitenvergleich ist auf der härteren im Vergleich zur weicheren Seite mit der Algometrie eine größere Kraft von $\geq 2$ Newton erforderlich um eine Schmerzreaktion auszulösen.

Patienten und Methode Im Rahmen einer sequentiellen statistischen Analyse (Bross) wurden Patienten mit einem einseitigen Myofaszialen Schmerzsyndrom bilateral mit der Strain-Elastografie (Ultrasonix Tablet - Analogic USA) an Nacken, Schulter, Rücken, Hüfte oder Beinen untersucht. Die Druckschmerzschwelle wurde mit einem Algometer (Model 320.1kN -TesT GmbH Deutschland) bestimmt. Mit der Software ImageJ wurden die Pixelanalyse der Elastografiebefunde durchgeführt.

Ergebnis In der sequentiellen statistischen Analyse zeigte sich nach 35 Probanden ein signifikantes Ergebnis mit Annahme der Nullhypothese. Auf der härteren Körperseite ist mit der Algometrie eine signifikant höhere Kraft zum Erreichen einer der Schmerzschwelle erforderlich.

Schlussfolgerung Triggerpunkt-Regionen der Myofaszie mit neurogene Entzündungen lassen sich mit der Strain-Elastografie abbilden. Damit eröffnet sich eine neue Dimension der Diagnostik und Behandlung Myofaszialer Schmerzen.

\section{12: Echo/Thorax/Notfall}

\section{F 15 Akzidentieller Pneumothorax infolge Ultraschall-gestützter supraclaviculärer Plexusblockade bei einer kachektischen Patientin mit distaler Radiusfraktur}

\author{
Autoren Oesterwind $K A^{1}$, Schöll $E^{1}$ \\ Institut 1 Universitätsspital Basel, Basel/CH \\ DOI 10.1055/s-0037-1606943
}

Patienten und Methode Zuweisung einer 89-jährigen kachektischen Patientin wegen distaler Radiusfraktur links infolge eines Stolpersturzes; Durchblutung, Motorik und Sensibilität der Hand waren unbeeinträchtigt. Die Patientin lebt selbständig im eigenen Haushalt mit 14-tägiger Pflegedienst-Unterstützung. Nach Rücksprache mit dem Traumatologen Entscheid für konservatives Vorgehen: Aushängen der Fraktur und manuelle Reposition in Regionalanästhesie. Ultraschallgestützte supraclaviculäre Plexusblockade links mit 20 ml Bupivacain $0,5 \%$, einmalige akzidentielle Luftaspiration unmittelbar nach Hautperforation, daraufhin Zurückziehen der Punktionskanüle und Fortführen der Blockade unter Sicht von Kanüle und umgebenden anatomischen Strukturen, keine Blutaspiration, keine Parästhesie. Reposition der distalen Radiusfraktur unter sonografischer Kontrolle. Die Patientin gab während und nach Interven- 
tion keine Beschwerden an, hatte jedoch trotz akzessorischem Sauerstoff nur eine $\mathrm{SpO} 2$ um $90 \%$.

Die postinterventionelle Thorax-Sonografie zeigte ein fehlendes Lungengleiten links sowie ein Barcode-Phänomen als klaren Hinweis auf einen Pneumothorax, welcher sich im Röntgenbild $(6,5 \mathrm{~cm})$ bestätigte. Einlage eines MatthysDrain links im 4. ICR in der mittleren Axillarlinie, worunter sich der Pneumothorax komplett regredient zeigte, im weiteren stationären Aufenthalt der Patientin aber rezidivierte, so dass er über zwei Tage mittels Bülau-Drainage therapiert werden musste. Nach dreiwöchigem Klinikaufenthalt konnte die Patientin in die ambulante Weiterbehandlung entlassen werden.

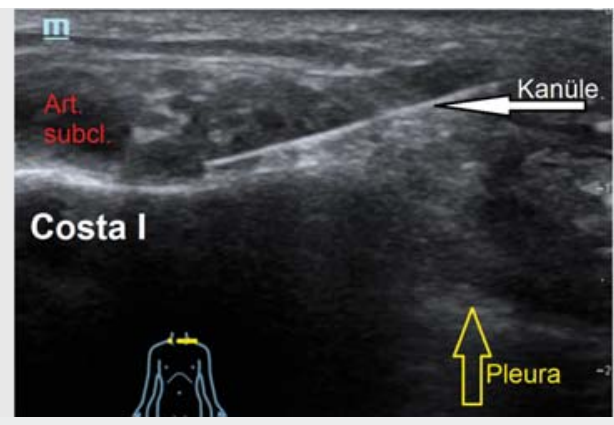

- Abb. 1 Punktionslokalisation bei kachektischer Patientin. Das Sonografie-Bild zeigt die Situation nach korrekter Platzierung der Kanüle zwischen erster Rippe und Plexus.
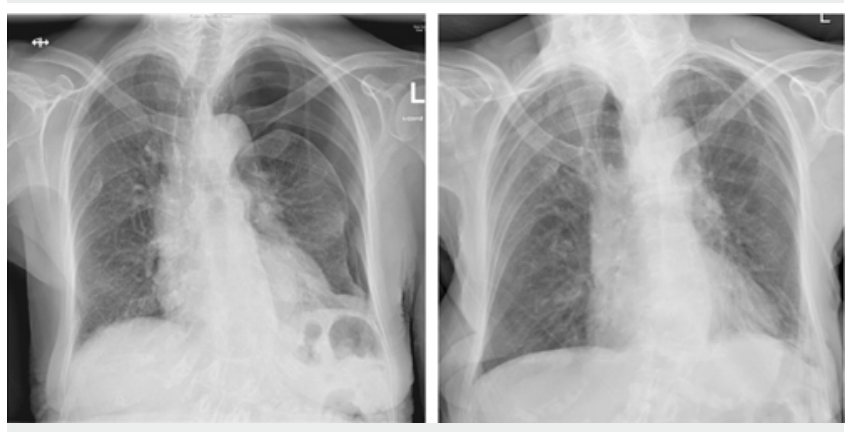

Abb. 2 Pneumothorax nach akzidentieller Pleurapunktion (linkes Bild) sowie Situation nach Matthys-Drain-Einlage (rechtes Bild)

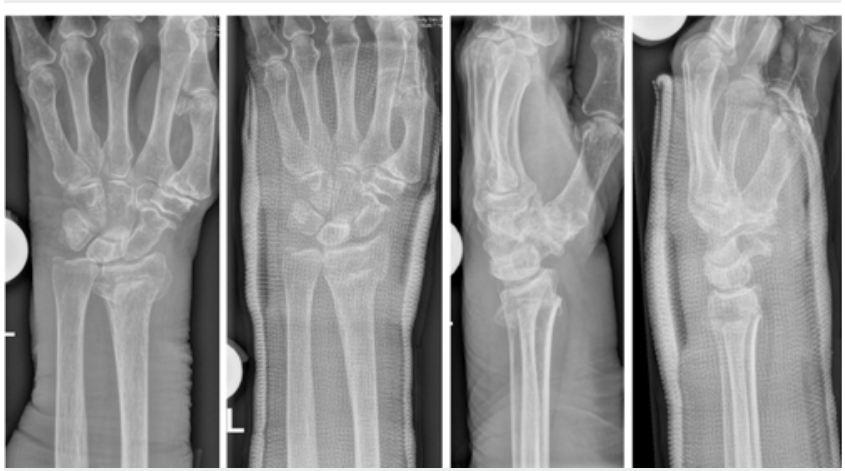

Abb. 3 ap und laterales Röntgenbild vor und nach Frakturreposition unter supraclaviculärer Plexusblockade

Schlussfolgerung Neueren Untersuchungen zufolge liegt die Häufigkeit eines akzidentiellen Pneumothorax unter periclaviculärer sonografisch gestützter Plexusblockade bei $0,05 \%$. Ohne Ultraschall-Führung der Punktionskanüle liegt die Rate indes bei bis zu 6\%. Unsere Komplikation lässt sich im Nachhinein mit dem plötzlichen Hautdurchtritt der atraumatischen Injektionskanüle (Facettenschliff $30^{\circ}$ ) bei kachektischer Patientin erklären. Für die Hautperforation wurde größerer Druck aufgewendet, welcher beim plötzlichen Durchtritt der Nadel zur Verletzung der darunterliegenden Pleura und Lunge geführt hat.

\section{V12.001 Ultraschall-gestützter supraclaviculärer Block zur Analgesie und Reposition distaler Radiusfrakturen auf der Notfallstation}

Autoren Schöll E ${ }^{1}$, Ammann $S^{2}$, Nieves Ortega $R^{1}$, Krähenbühl $G^{1}$, Bingisser $\mathrm{R}^{1}$

Institut 1 Universitätsspital Basel, Basel/CH; 2 Universität Basel, Basel/CH DOI 10.1055/s-0037-1606944

Problemstellung Distale Radiusfrakturen machen 25\% aller Knochenbrüche bei Erwachsenen aus und sind damit die häufigste Frakturart. Ältere Patienten sind öfter betroffen, werden indes zu 85\% konservativ behandelt. RepositionsManöver auf Notfallstationen (NFS) werden meist unter intravenöser- oder Bruchspalt-Analgesie durchgeführt. Supraclaviculäre Regionalanästhesie (SCRA) ist eine weitere Methode zur Schmerzbefreiung bei Radiusfrakturen. Unser Ziel war, SCRA als Standardprozedur bei diesen Frakturen einzuführen. Patienten und Methode Patienten (>18 Jahre) mit distaler Radiusfraktur, welche nicht unmittelbar nach Eintritt auf der NFS für eine Operation vorgesehen waren, wurde die SCRA als alleinige Analgesie zur Frakturreposition angeboten. Kontraindikationen waren Allergien auf Lokalanästhetika oder Infektionen im Punktionsbereich. Für die sonografische Visualisierung des supraclaviculären Plexus sowie der Punktionskanüle wurde ein MINDRAY TE7 mit einer linearen Sonde L16-4Hs verwendet. Der Plexus wurde oberhalb der ersten Rippe dargestellt und unter sterilen Kautelen sowie In-plane-Sicht der Punktionskanüle mit $20 \mathrm{ml}$ Bupivacain 0,5\% umspült. Nach Eintritt der anästhetischen Wirkung erfolgten eine 15-minütige Traktion sowie die manuelle Reposition ggf. unter Ultraschall-Kontrolle.

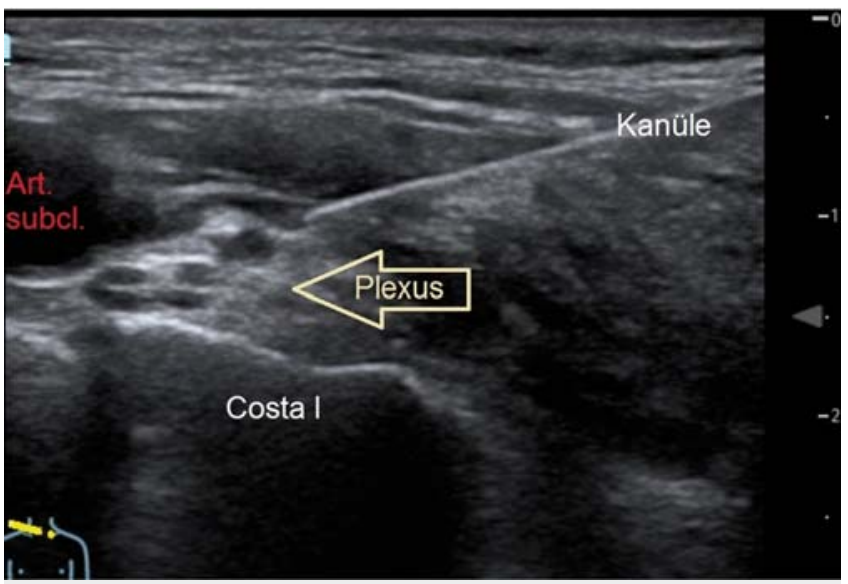

Abb. 1 Schema der supraclaviculären topografischen sowie sonografischen Anatomie mit Interventionsnadel

Ergebnis Von 02/2014 bis 04/2017 wurden 62 Patienten (w55; m7), mittleres Alter 71 Jahre ( $\min 21$; $\max 95)$ unter SCRA auf unserer NFS reponiert. Die SCRA wurde von zwei Oberärzten und 8 supervidierten Assistenzärzten durchgeführt. Post-interventionem wurden 34 Patienten ambulant entlassen oder in eine Pflegeeinrichtung überwiesen, 28 Patienten wurden stationär behandelt, wovon 25 operiert wurden. 

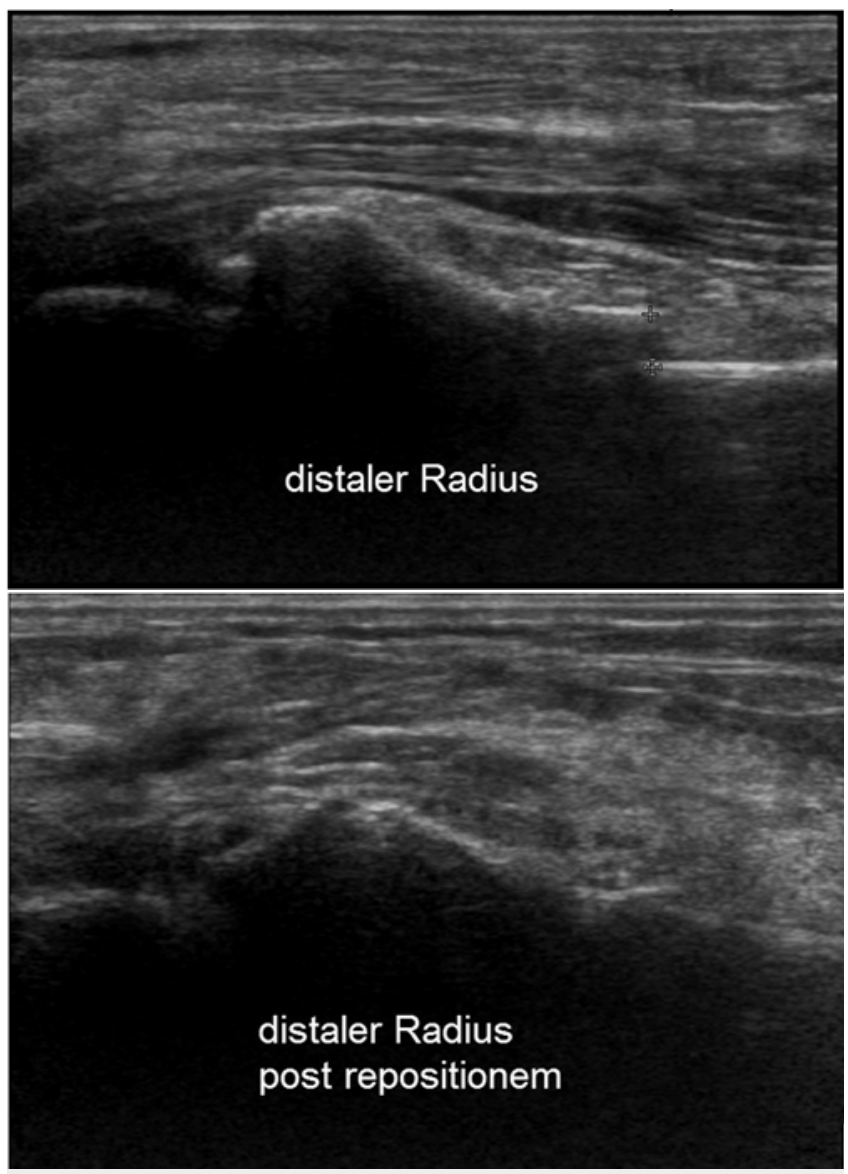

- Abb. 1 Volare Ansicht der sonografisch kontrollierten Reposition: oben vor und unten nach Manipulation

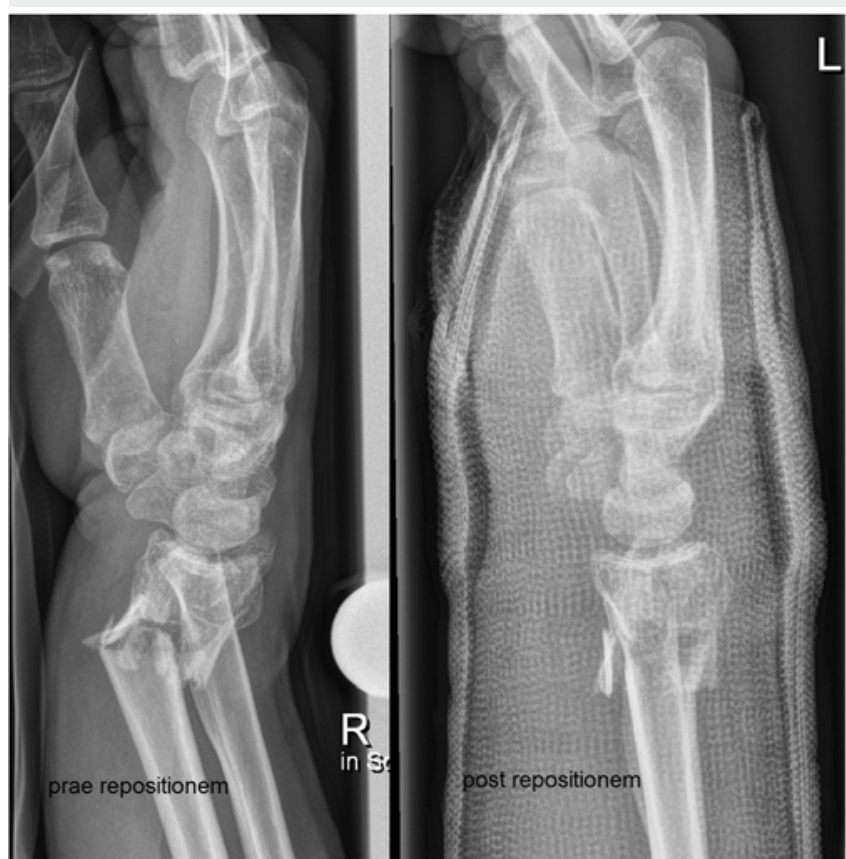

Abb. 2 Konventionelles laterales Röntgenbild vor und nach Reposition unter SCRA

Als einzige interventionsbezogene Komplikation war ein iatrogener Pneumothorax zu verzeichnen, welcher durch eine Thoraxdrainage therapiert werden musste und zum verlängerten stationären Aufenthalt führte.
Schlussfolgerung Ultraschall-gestützte SCRA auf der NFS ist eine zufriedenstellende Methode zur Analgesie und Reposition distaler Radiusfrakturen.

\section{V12.002 Orbitalphlegmone im Kindesalter: Stellenwert der Ultraschalldiagnostik zur Diagnosesicherung und zum Monitoring - Erfahrungen der letzten 10 Jahre}

Autoren Klee $\mathrm{D}^{1}$, Yu $\mathrm{Y}^{1}$, Schaper $\mathrm{J}^{1}$

Institut 1 Heinrich-Heine Universität Düsseldorf, Medizinische Fakultät, Düsseldorf/DE

DOI 10.1055/s-0037-1606945

Problemstellung Zur Diagnostik bei der Fragestellung „Orbitalphlegmone“ werden die Orbitasonografie (OS), die Computertomografie (CT) und die Magnetresonanztomografie (MRT) eingesetzt. Ziel dieser Studie war es, die Wertigkeit der OS als primär bildgebendes Verfahren zur Diagnosesicherung und zur Verlaufskontrolle zu überprüfen.

Patienten und Methode 124 Kindern (70 34 o; Alter: 4,2 54,2 Jahre) mit akuter Lidschwellung und der Fragestellung nach Orbitalphlegmone wurden retrospektiv anhand der Kriterien präseptale Weichteilvermehrung, postseptale Weichteilvermehrung, Abszessformationen und Lamina papyracea Defekte analysiert. Retrospektiv wurde eine Einteilung der Ergebnisse nach Jain und Rubin vorgenommen.

Ergebnis Bei 118 Patienten erfolgte als primär bildgebende Diagnostik die OS, drei Patienten erhielten primär eine CT und drei weitere eine MRT.

Von insgesamt 124 vorgestellten Kindern zeigten primär 34\% eine postseptale Weichteilvermehrung (Gruppe 2 nach Jain und Rubin) und 9\% eine Abszessformation (Gruppe 3). Bei den meisten Kindern waren präseptale Weichteilvermehrungen (Gruppe 1) oder andere Veränderungen außerhalb der Orbitae ursächlich für ein geschwollenes Auge.

Die Patienten der Gruppe 2/3 erhielten eine intravenöse antibiotische Therapie; im Durchschnitt erfolgte am dritten Tag eine Verlaufsdiagnostik. Dabei wurden im Mittel drei bildgebende Kontrollen durchgeführt (82\% OS, 10\% MRT, 8\% CT). Aufgrund klinischer oder sonografischer Verschlechterung des Befundes war bei 19 Patienten, die primär eine OS erhielten, weitere bildgebende Diagnostik (MRT/CT) indiziert. Im Verlauf war bei zehn Patienten eine invasive Therapie notwendig.

Schlussfolgerung Die OS ist als Verfahren zum Management bei „unklare Lidschwellung“ mit der Fragestellung Orbitalphlegmone geeignet. Die OS ist als primäres diagnostisches Verfahren bei „unklarer Lidschwellung“ immer geeignet und in den meisten Fällen als einziges bildgebendes Verfahren ausreichend.

\section{V12.003 Quantifizierung der diastolischen} Dysfunktion via Altersabhängigkeit der diastolischen Myokardfunktion - Einfluss der Insulin Resistenz ohne und bei Typ 2 Diabetes

\author{
Autoren Bibra $\mathrm{H}_{\text {von }}{ }^{1}$, Leclerque $\mathrm{C}^{1}$, Siegmund $\mathrm{T}^{1}$ \\ Institut 1 Städt. Krhs München-Bogenhausen, München/DE \\ DOI 10.1055/s-0037-1606946
}

Problemstellung Die alarmierende Prävalenz und Prognose von Herzversagen mit erhaltener Ejektions Fraktion verlangt eine Quantifizierung der diastolischen Dysfunktion (DDF). Die myokardiale diastolische Geschwindigkeit (E') ist durch das Lebensalter dominant determiniert.

Hypothese: Diese Abhängigkeit ermöglicht eine Quantifizierung der DDF sowie Bestimmung ihrer altersunabhängigen strukturellen und metabolischen Determinanten.

Patienten und Methode Diese prospektive Querschnittstudie analysierte kardiovaskuläre, metabolische und Echokardiografie-/Gewebedoppler-/CarotisSono Daten von 409 konsekutiv im Diabetes Center, Klinikum München-Bogenhausen, untersuchten Patienten zwischen 20 und 90 Jahren, in Sinusrhyth- 
mus, ohne bekannte Herzerkrankung und entweder mit Typ-2-Diabetes (D, $n=204)$ oder ohne $D$ als Kontrollgruppe $(K, n=209)$, unter Einschluss einer Subgruppe normaler Individuen ohne kardiovaskuläre Risikofaktoren ( $\mathrm{N}$, $n=94)$.

Ergebnis E' korrelierte hochsignifikant mit dem Lebensalter in $\mathrm{N}$ : E' ${ }_{\text {norm }}=$ $-0,16^{*}$ Jahre $+20\left(R^{2}=0,77 ; p<0,0001\right)$, bei $1 \%$ Reduktion pro Lebensjahr. Deshalb wurde DDF quantifiziert als Defizit E' - E' ${ }_{\text {norm }}$ und definiert als $>95 \%$ Konfidenzintervall. Im Vergleich zu K hatten Diabetiker niedrigeres E' bei höherem Alter, BMI, kardiovaskulären Risiko und DDF (28\% vs. 6\%). In K korrelierte die Schweregradeinteilung von DDF (anhand E'-E' ${ }_{\text {norm }}$ ) mit der des LV Füllungsdrucks E/E'. Multivariate Analyse bestätigte als Determinanten für DDF die Pulswellengeschwindigkeit, den diastolischen Blutdruck und den Triglyzerid/HDL Quotienten (Marker der Insulin Resistenz) für K und in umgekehrter Reihenfolge für Diabetiker. LA Größe oder LV Masse waren keine signifikante Determinanten für DDF.

Schlussfolgerung Der physiologische Einfluss des Lebensalters reduziert E' um $1 \%$ pro Jahr und ermöglicht die Quantifizierung der diastolischen Dysfunktion und die Ermittlung altersunabhängiger Risikofaktoren, wobei insbesondere Insulin Resistenz als metabolisches Risiko für DDF entlarvt wird.

\section{V12.004 Diagnostische Möglichkeiten einer detaillierten echokardiografischen Beurteilung des fetalen Herzens im 1. Trimenon - eine Standortbestimmung}

Autoren Weichert $\mathrm{J}^{1}$, Gembicki $\mathrm{M}^{1}$, Fernandes $\mathrm{T}^{1}$, Hartge $\mathrm{D}^{1}$ Institut 1 Universitätsklinikum Schleswig-Holstein, Lübeck/DE DOI 10.1055/s-0037-1606947

Problemstellung Evaluation aktueller und neuartiger diagnostischer Möglichkeiten der fetalen Echokardiografie im 1. Trimenon im Hinblick auf diagnostischen Nutzen und Reproduzierbarkeit in einem low-risk-Kollektiv.

Patienten und Methode Im Rahmen dieser prospektiven Studie wurden 174 unkomplizierte Einlingsschwangerschaften analysiert von denen alle einem 3stufigen echokardiografischen Untersuchungsansatz unterzogen worden. Die frühe Echokardiografie erfolgte ausnahmslos transabdominal, hierbei wurde neben der Quantifizierung kardialer Kenngrößen auch das Farbdopplermuster ausgewertet. Darüber hinaus erfolgte eine volumetrische 4D-Aufnahme des Fetalherzens mit nachfolgender semiautomatisierter workflow-basierter Analyse mittels 5DHeart-Technologie zur vergleichenden Darstellung der Möglichkeit der Rekonstruktion aller diagnostischen Herzebenen.

Ergebnis Insgesamt konnten die Daten von 164 Feten ausgewertet werden. Das mittlere Gestationsalter (GA) lag bei 12,9 SSW (11,0 bis 14,0 SSW). In 157/ 164 (96\%) Fällen konnten die Messparameter vollständig erhoben werden (Herz-/Thoraxdurchmesser, -fläche, Herzachse, LVOT/RVOT). In 100\% konnte das Farbdopplermuster (Ein-/Ausfluss) erfasst werden. In 4 Fällen fiel ein von Typ 1 abweichendes Füllungsmuster auf (jeweils 2 Feten Typ 2 bzw. Typ 3). Die Herzachse lag bei allen Feten im Normalbereich (36,5 bis $52,0^{\circ}$ ). Während der zusätzlichen 4D-Sonografie wurden 1 bis 4 Volumendatensätze pro Fet aufgenommen. In 118/164 Fällen (72\%) konnten $\geq 6$ Herzebenen visualisiert werden, in $>1 / 3$. konnten sogar alle oder zumindest $8 / 9$ diagnostischen Ebenen adäquat rekonstruiert werden. Mittels offline-Analyse war es möglich, in 92\% (150/164) vergleichbare Informationen zu erheben wie mit der konventionellen 2D-Echokardiografie.

Schlussfolgerung Sowohl die Registrierung des Farbdopplermusters, als auch die standardisierte volumetrische Analyse des Fetalherzens (5DHeart) sind geeignete Methoden zusätzliche, wertvolle Informationen zu anatomischen Integrität des frühen fetalen Herzens zu liefern, auch bereits zum Ende des 1. Trimenons.

\section{V12.005 Modellierung von PV Loops für LV basierend auf 3D-Echokardiografie}

Autoren Winkler $C^{1}$, Linden $K^{1}$, Recker $F^{1}$, Breuer $J^{1}$, Herberg $U^{1}$

Institut 1 Universitätsklinikum Bonn, Bonn/DE

DOI 10.1055/s-0037-1606948

Problemstellung Therapie und Krankheitsverlauf von Patienten mit angeborenen Herzfehlen sind komplex und abhängig von der individuellen Anatomie und Funktion des Herzens. Um Behandlungen und Eingriffe zu planen, werden meistens Blutdruckmessungen im LV (linken Ventrikels) benötigt, die bisher nur aus invasiven Messungen gewonnen werden können. Ziel dieser Studie ist es, die Blutdruckverläufe im LV mittels computergestützter Herzkreislaufmodellen und nichtinvasiver 3D-Echokardiografie (3DE) zu berechnen. Somit lassen sich patienten-individuelle Druck-Volumenschleifen (PV Loops) approximieren, die bei Diagnose und Planung von Eingriffen helfen.

Patienten und Methode Es wurde ein Programm entwickelt, das die Simulation von Druck- und Volumenverläufe im LV ermöglicht. Die Grundlage des Programms ist ein physikalisches Strömungsmodell des Herzkreislaufsystems (Abb. 1). Die individuelle Anpassung der Modelle erfolgt mit Volumenzeitkurven, die aus den 3DE-Daten ermittelt wurden. Resultierend aus der Anpassung wird ein Datensatz von Herzparametern bestimmt, der das Verhalten des patienten-individuellen Kreislaufs definiert.

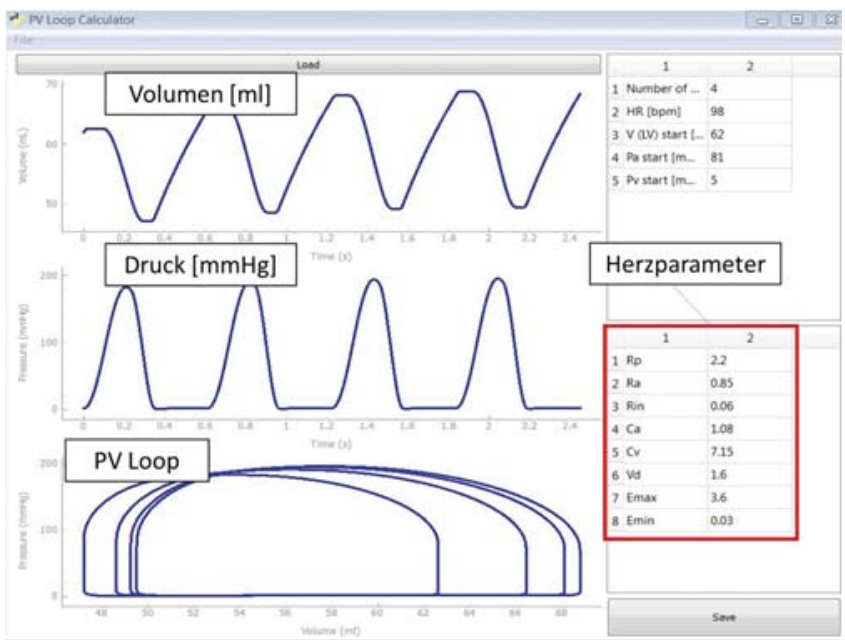

Abb. 1 Programm zur Simulation von Volumen, Druck und PV Loops

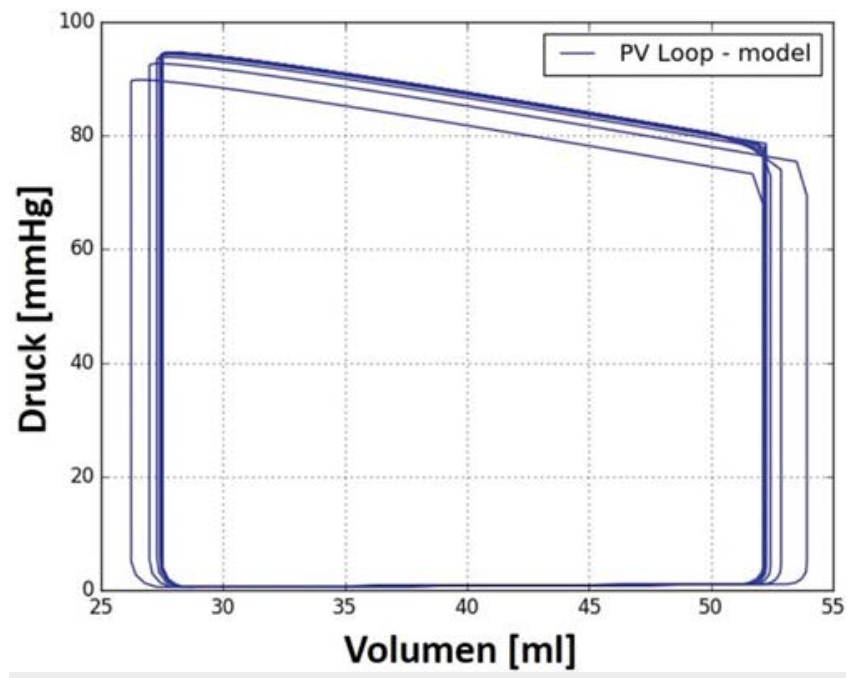

Abb. 2 Individuell angepasste PV Loops für eine gesunde Untersuchungsperson 


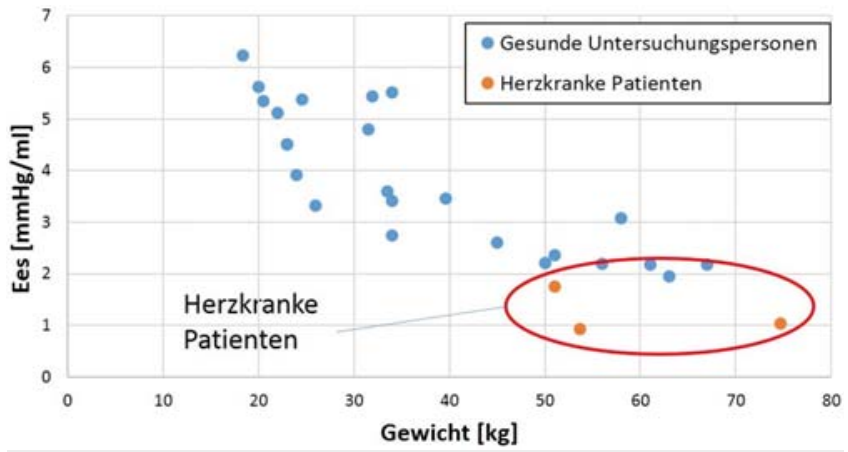

Abb. 3 Ees für 24 gesunde und 3 kranke Kinder gegen das Gewicht aufgetragen

Ergebnis In einem ersten Test wurden die Modelle an die 3DE-Daten von 24 gesunden Kindern und 3 univentrikulären Herzkranken angepasst. Als Ergebnis konnten PV Loops berechnet werden (Abb. 2). Zur Validierung werden invasiv gemessene PV Loops der Patienten herangezogen. Aus den angepassten Modellen können beispielsweise die endsystolische Elastanz (Ees) für LV bestimmt werden (Abb. 3). Abbildung 3 zeigt, dass Ees von Gewicht der Untersuchungsperson abhängig ist. Zudem konnten wir einen Unterschied zwischen gesunden und kranken Kindern bezüglich Ees feststellen.

Schlussfolgerung Die vorgestellten Modelle ermöglichen die Approximation von PV Loops und Herzparametern mittels nichtinvasiven Messungen. Dieser Ansatz kann Ärzten helfen klinische Parameter zu berechnen und invasive Messungen zu vermeiden. Im Folgenden müssen die Ergebnisse auf Validität überprüft werden.

\section{V12.006 B-Linien und comet tail artifacts als Ultraschall-Reverberationsartefakte am Rande von Lungenkonsolidierungen - eine retrospektive quantitative und qualitative Analyse}

\author{
Autoren Reifeltshammer $C^{1}$, Mathis $G^{2}$ \\ Institut 1 MUW, Wien/AT; 2 Internistische Praxis, Rankweil/AT \\ DOI 10.1055/s-0037-1606949
}

Problemstellung In der „International Consensus Conference on Lung Ultrasound (ICC-LUS)“" von 2012 wurde folgende Aussage über das fokale interstitielle Syndrom getroffen:

"Visualization of a focal B-line pattern can be suggestive of the presence of an adjacent pulmonary consolidative process, as in the case of pneumonia, infarction, contusion or cancer."

Über das Ausmaß und die Häufigkeit des Auftretens eines fokalen interstitiellen Syndroms im Rahmen der oben genannten Konsolidierungen gibt es sehr wenig Literatur.

Patienten und Methode In dieser retrospektiven Studie wurde geprüft, ob sich 4 Arten von Lungen-Konsolidierungen unterscheiden in der Anzahl, der Verteilung und dem Erscheinungsbild von B-Line-Ultraschallartefakten, die angrenzend zur jeweiligen Konsolidierung im Rahmen des fokalen interstitiellen Syndroms entstehen. Ebenso wurde das Auftreten von comet-tail-Artefakten untersucht.

Es wurden 127 Ultraschall-Aufnahmen von Konsolidierungen ausgewertet, davon 52 Pneumonien, 41 Pulmonalembolien,29 Lungentumore, 5 Lungenkontusionen.

Ergebnis Es zeigte sich, dass 71,7\% aller untersuchten Konsolidierungen keine B-Lines aufwiesen, bei 23,6\% waren es lediglich 1-2 B-Lines. 3,9\% der Konsolidierungen wiesen 3-10 B-Lines auf, 0,8\% mehr als 10 B-Lines.

Hinsichtlich der comet-tail-Artefakte in der Tiefe zeigte sich, dass diese in 35,4\% der Fälle vorhanden waren. 37\% der Konsolidierungen zeigten keine comet-tail-Artefakte und $27,6 \%$ der Konsolidierungen wiesen eine dorsale Pseudoschallverstärkung auf.

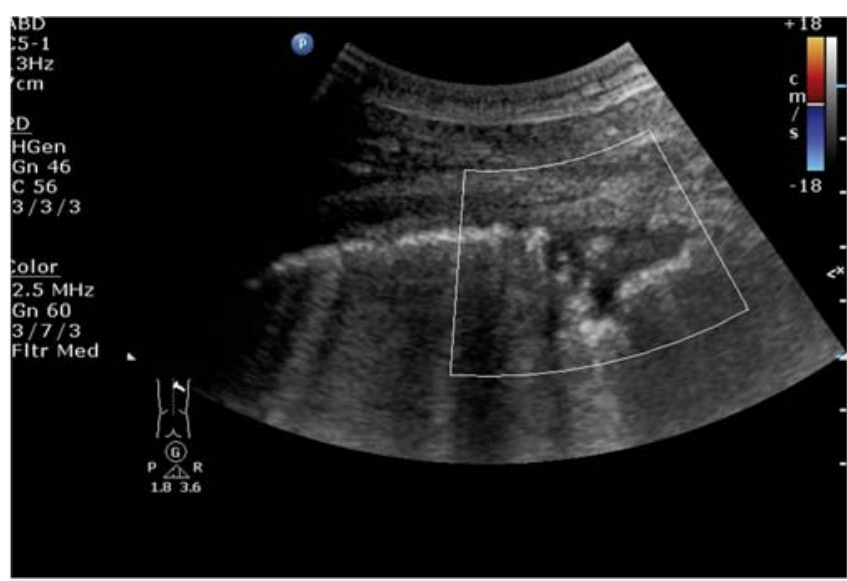

Abb. 1 fokales interstitielles Syndrom bei einer Pneumonie
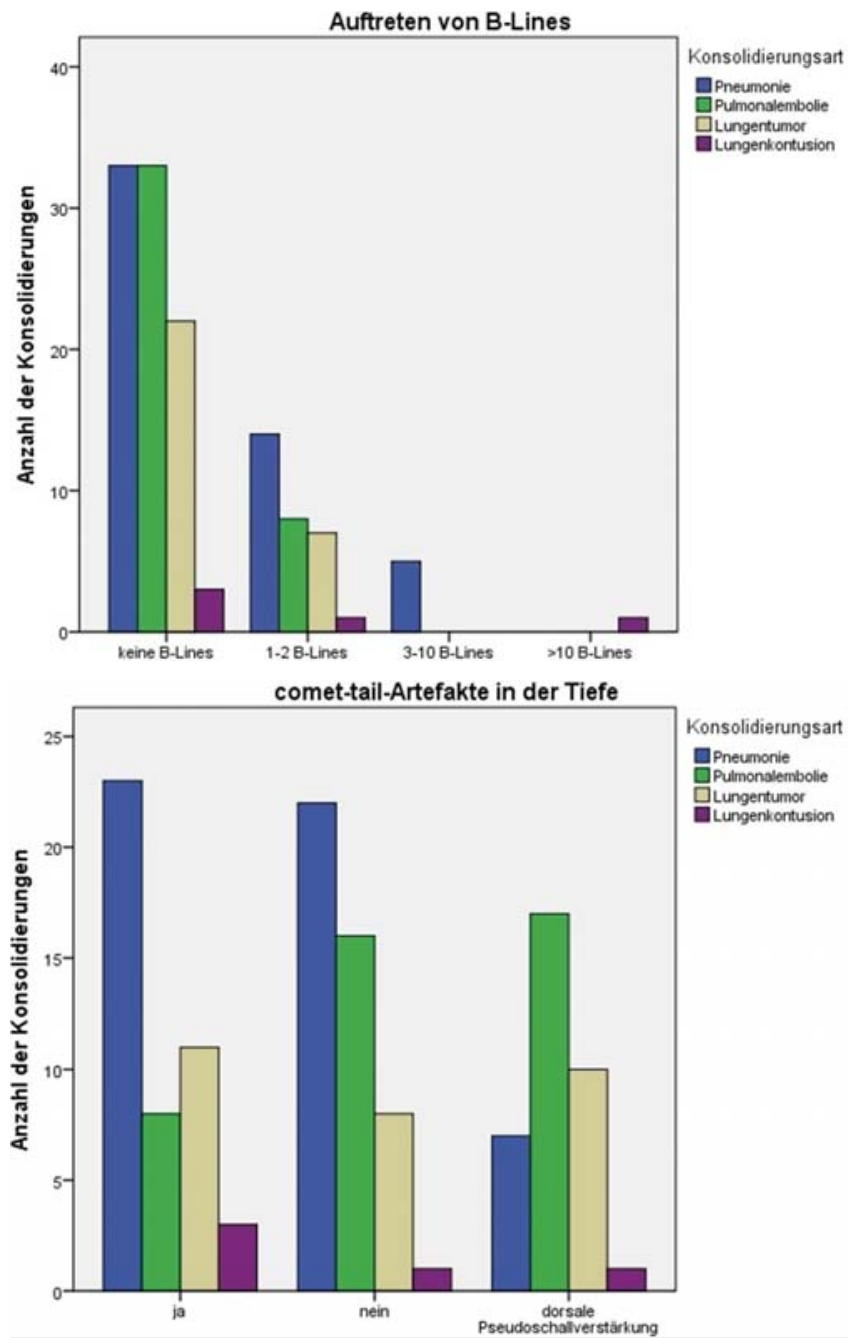

Abb. 1 Auftreten von B-Lines \& Auftreten von comet-tail-Artefakten

Schlussfolgerung Definiert man das fokale interstitielle Syndrom als ein Auftreten von zumindest 3 B-Lines, so ist dieses mit 4,8\% ein seltener Befund bei den untersuchten Konsolidierungen. Comet-tail-Artefakte als Zeichen interstitieller Flüssigkeitseinlagerung fehlen ebenso in $64,6 \%$ der untersuchten Konsolidierungen. 


\section{V12.007 Vaskularisation primärer maligner peripherer Bronchialkarzinomen in der CEUS- retrospektive Studie an $\mathbf{n}=89$ Patienten}

Autoren Findeisen $\mathrm{H}^{1}$, Trenker $\mathrm{C}^{1}$, Figiel $]^{1}$, Görg $\mathrm{K}^{1}$, Greene $\mathrm{B}^{2}$, Görg $\mathrm{C}^{1}$ Institut 1 Universitätsklinikum Marburg, Marburg/DE; 2 Philipps-Universität Marburg, Marburg/DE

DOI 10.1055/s-0037-1606950

Problemstellung Die Lunge ist durch eine duale Gefäßvaskularisation - pulmonalarteriell (PA) und bronchialarteriell (BA) - charakterisiert. Die Tumorneoangiogenese geht prinzipiell von den BA aus. Es ist unklar, ob alle Bronchialkarzinome (BC) diesen Vaskularisationtyp zeigen.

Patienten und Methode Im Zeitraum von April 2004 bis September 2015 wurden $\mathrm{n}=89$ Patienten mit peripherem BC zunächst im B-Bild untersucht (Airbronchogramm; $n=17$, Thoraxwandinfiltration; $n=17$ ). In der CEUS wurde Ausmaß (EE) (hypoechogen/isoechogen/hyperechogen), Homogenität (HE) (homogen/inhomogen/Nekrose; $n=42$ ) und die Zeit zum Enhancement (TE) bestimmt. Ein vorzeitiges Enhancement (PA) im Vergleich zur Thoraxwand wurde von einem zeitgleichen Enhancement (BA) unterschieden. CEUS-Daten wurden mit B-Bild Sonografie und Histologie verglichen.

Ergebnis $n=25$ Patienten (28\%) hatten ein vorzeitiges PA-Enhancement (TE: $8 \pm 3,7 \mathrm{~s}), \quad n=64$ (72\%) ein zeitgleiches BA-Enhancement (TE: 17,6 $\pm 6,2 \mathrm{~s})(\mathrm{p}<0,001)$. Das PA-Enhancement (EE/HE) war hyperechogen $(n=11 /$ $25)$, homogen $(n=11 / 25)$ und zeigte im B-Bild häufiger ein Airbronchogramm $(n=11 / 17, p<0,001)$. Das BA-Enhancement $(E E / H E)$ war bevorzugt hypoechogen $(n=34 / 64)$ und inhomogen $(n=54 / 64)$. Bei einem BA-Enhancement fanden sich häufiger Nekrosen $(n=36 / 42, p=0,009)$ und Thoraxwandinfiltrationen $(n=16 / 17, p=0,033)$. PA- und BA-Enhancement verteilten sich in den unterschiedlichen Histologien: $n=42$ Adenokarzinome (18 PA, 24 $\mathrm{BA}$ ), $\mathrm{n}=30$ Plattenepithelkarzinome ( $4 \mathrm{PA}, 26 \mathrm{BA}$ ), $n=13$ sonstige NSCLC ( 3 PA, $10 \mathrm{BA})$, und $n=4$ SCLC (0 PA, $4 \mathrm{BA})(p=0,016)$.

Schlussfolgerung Die Vaskularisation von peripheren $B C$ ist heterogen und wird durch Histologie und B-Bild-Parametern beeinflusst. Es zeigt sich neben einer vorherrschend bronchialarteriellen Versorgung von malignen Lungentumoren auch ein Anteil von Adenokarzinomen und Nicht-Adenokarzinomen, die ein PA-Enhancement zeigen.

\section{V12.008 ARFI-Elastografie der Lunge - eine neue Methode in der Lungenfunktionsdiagnostik?}

Autoren Klinger $C^{1}$, Kruse $A^{1}$, Grotz $A^{1}$, Fröhlich $E^{2}$, Riecken $B^{1}$ Institut 1 Klinikum Ludwigsburg, Ludwigsburg/DE; 2 Universität Tübingen, Tübingen/DE

DOI 10.1055/s-0037-1606951

Problemstellung Erste Studien weisen auf einen möglichen Stellenwert elastografischer Ultraschallverfahren bei der Detektion subpleural gelegener fokaler Lungenveränderungen hin. Untersuchungen zum Nutzen bei diffusen Lungenerkrankungen gibt es bisher nicht. Ziel dieses Pilotprojekts ist der Vergleich der mittels ARFI-Elastografie bestimmten Lungensteifigkeit von Lungengesunden und Patienten mit schwerer COPD.

Patienten und Methode Von Februar 2017 bis Mai 2017 wurden 10 Lungengesunde und 10 COPD-Patienten ( $\geq$ GOLD III) mittels ARFI-Elastografie (Siemens Acuson S3000; Virtual TouchTM Tissue Quantification) untersucht. Alle Messungen wurden rechtsthorakal mit einer 6C1HD-Schallsonde in MonaldiPosition (2. ICR, Medioklavikularlinie) ca. $1 \mathrm{~cm}$ subpleural senkrecht zur Lungenoberfläche durchgeführt. Bei jedem Patienten wurde $10 \mathrm{Mal}$ in maximaler In- und Exspiration gemessen.

Ergebnis Beide Gruppen waren bezüglich Alter und Geschlecht vergleichbar (72,1 $\pm 10,3$ Jahre vs. 67,9 \pm 11 , 1 Jahre; je $80 \%$ männlich). Die Werte in Exspiration waren bei Lungengesunden signifikant niedriger $(1,78 \mathrm{~m} / \mathrm{s}$ vs. $2,98 \mathrm{~cm} / \mathrm{s}, \mathrm{p}<0,01$ ) als bei COPD-Patienten (Abb. 1). Die Werte in Inspiration unterschieden sich nicht $(3,52 \mathrm{~m} / \mathrm{s}$ vs. $3,2 \mathrm{~m} / \mathrm{s}, \mathrm{p}=0,9)$. Im Gegensatz zu den COPD-Patienten $(p=0,8)$ unterschieden sich die Werte in Inspiration und Ex- spiration in der Gruppe der Lungengesunden signifikant $(p<0,001)$. Bei Betrachtung der individuellen Patientendaten zeigte sich, dass im Gegensatz zu den COPD-Patienten ein Großteil der Lungengesunden in Inspiration höhere Messwerterte aufwies als in Exspiration (Abb. 2).

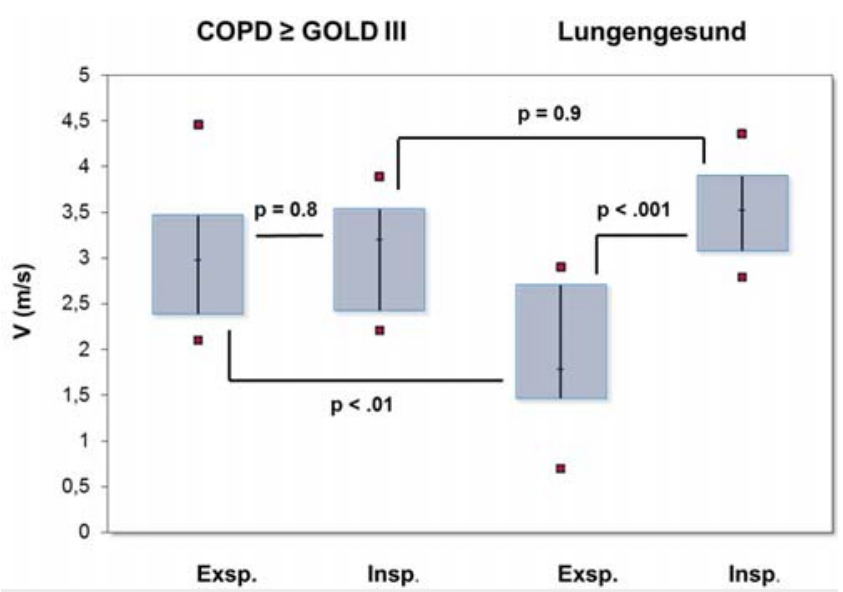

Abb. 1 Boxplots der ARFI-Messwerte in max. Exspiration und Inspiration.

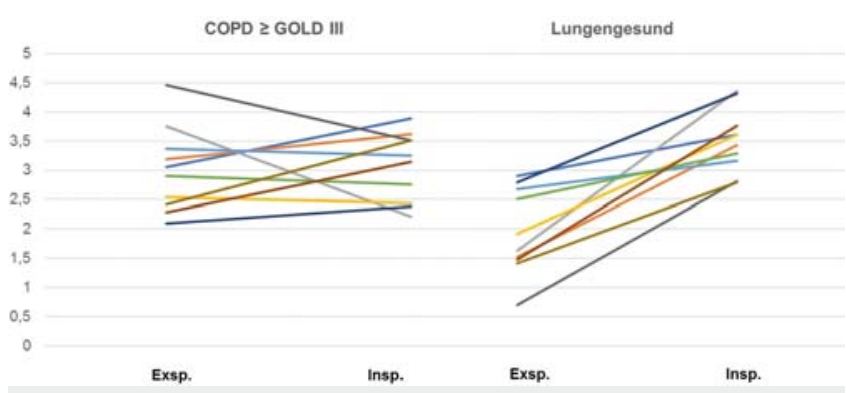

Abb. 2 Mediane individuelle ARFI-Messwerte in max. Exspiration und Inspiration.

Schlussfolgerung Mittels ARFI der Lunge können Lungengesunde von Patienten mit schwerer COPD unterschieden werden. Weitere Untersuchungen sollten Normwerte für Lungengesunde definieren und evaluieren, ob elastografische Parameter mit Parametern der Bodyplethysmografie korrelieren.

\section{V12.009 Der Einsatz von Ultraschall im mobilen Palliativteam}

Autoren Ausserer $\mathrm{C}^{1}$, Schwärzler $\mathrm{B}^{2}$, Gehmacher $\mathrm{O}^{2}$

Institut 1 LKH Hohenems, Wolfurt/AT; 2 LKH Hohenems, Hohenems/AT

DOI 10.1055/s-0037-1606952

Problemstellung Die Mehrzahl, der vom MPT betreuten Patienten hat eine Lebenserwartung von Tagen bis wenige Monate. In dieser Phase steht vor allem eine gute Symptomkontrolle an oberster Stelle. Mit dem mobilen Ultraschallgerät steht dem Palliativmediziner ein gutes diagnostisches Mittel zur Verfügung, die Symptomursache - meist Atemnot, Schmerz oder abdominelle Probleme - abzuklären, sowie Therapien wie Aszites- oder Pleurapunktionen zuhause durchführen zu können. Hierdurch bleibt den Patienten der oft beschwerliche Weg ins Krankenhaus erspart.

Patienten und Methode Von Jänner 2016 bis inklusive Jänner 2017 wurde eine prospektive Beobachtungsstudie durch das MPT Hohenems durchgeführt. Hierbei wurden der mobile Ultraschall hinsichtlich Indikation, Befund, therapeutischer Konsequenz und Komplikationen untersucht. In den 13 Monaten wurden 110 Patienten untersucht.

Ergebnis Beim Patientenkollektiv handelte es sich mehrheitlich um Patienten mit einer malignen Grunderkrankung $(n=92)$. Die Hauptindikation für den 
Ultraschall waren Atemnot $(n=53)$ und Schmerz $(n=35)$. Ansonsten standen hauptsächlich abdominelle Beschwerden im Vordergrund. Bei 45 Patienten wurde ein Pleuraerguss und bei 61 Patienten Aszites festgestellt. Als therapeutische Konsequenz wurden 29 Pleura- und 34 Aszitespunktionen durchgeführt. In lediglich 5 Fällen kam es dabei zu leichtgradigen Komplikationen. Insgesamt konnten 68 stationäre Aufnahmen verhindert werden. 3 Patienten wurden aufgrund des Ultraschallbefundes ins Krankenhaus überwiesen. In 12 Fällen wurde die medikamentöse Therapie geändert.

Schlussfolgerung Die Sonografie stellt eine nicht invasive Untersuchung dar, die es dem Palliativmediziner erlaubt, schwer kranke Patienten zuhause zu behandeln. Meist kann durch die Ultraschalluntersuchung als direkte Konsequenz eine Therapie wie z.B. eine Aszites- oder Pleurapunktion mit geringer Komplikationsrate durchgeführt werden. Dies erspart den Patienten, die sich in der letzten Phase ihres Lebens befinden, in der Mehrzahl der Fälle den beschwerlichen Transport ins Krankenhaus.

\section{2: Pädiatrie I}

\section{V2.001 Vereinfachte Ultraschall-Klassifikation der Säuglingshüfte nach Graf gemäss diagnostisch-therapeutischen Richtlinien}

\begin{abstract}
Autoren Schmid $\mathrm{R}^{1}$, Baumann $\mathrm{T}^{2}$, Essig $\mathrm{S}^{2}$, Munkuu $\mathrm{B}^{3}$
\end{abstract}
Institut 1 Baarer Kinderarztpraxis, Baar/CH; 2 Institut für Hausarztmedizin und Community Care, Luzern/CH; 3 National Center for Maternal and Child Health, Ulaanbaatar/MN

DOI 10.1055/s-0037-1606953

Problemstellung Basierend auf den Erfahrungen des SwissMongolian Pediatric Projects (von 2012 bis 2016 über 200'000 Untersuchungen und 3'500 erfolgreiche Therapien) und den Resultaten einer prospektiven Kohortenstudie, setzen wir im Rahmen des nationalen Ultraschall-Screeningprogrammes für Hüftdysplasie (DDH) eine neuartige, diagnostisch-therapeutische Gruppeneinteilung ein.

Patienten und Methode Es handelt sich um eine Adaptation des Graf-Systems in der Anwendung bei Neugeborenen. Nach therapeutischen Gesichtspunkten fassen wir die verschiedenen Hüfttypen zu lediglich 4 Gruppen zusammen. Gruppe A-Hüften sind nach Graf-Kriterien gesund und werden nicht weiter verfolgt; B-Hüften sind physiologisch unreif und benötigen eine Ultraschallbasierte Nachkontrolle um eine Progression zur DDH auszuschließen; C-Hüften sind dysplastisch und erhalten unmittelbar eine Behandlung mittels Flexions-Abduktions-Orthese; D-Hüften sind dysplastisch und der Femurkopf luxiert mit interponiertem Pfannendachknorpel, sie benötigen - nach initialem Versuch mit Orthese - meist eine chirurgische Versorgung.

Ergebnis Die ABCD-Einteilung der Säuglingshüfte wurde in fast der ganzen Mongolei eingeführt. In Grund- und Aufbaukursen für Screener wird die adaptierte Methode gelehrt. Inzwischen wird sie von rund 200 Anwendern in 23 Kliniken auf 36 digitalen Ultraschallgeräten eingesetzt. Da mit wenigen Ausnahmen alle Graf-Typen der DDH mittels einfacher Abspreizbehandlung in Sitz-Hock-Stellung zur Ausreifung gebracht werden konnten, erwies sich die strenge Einteilung nach Graf-Typen für die Anwender als zu kompliziert und für unser Konzept als nicht relevant.

Schlussfolgerung Für die Anwender der Hüftsonografie ist die neue Klassifikation eine erhebliche und plausible Vereinfachung.

\section{V2.002 Qualitätskonzept eines Ultraschall-basierten, nationalen Screeningprogramms für Hüftdysplasie in der Mongolei}

\author{
Autoren Essig $\mathrm{S}^{1}$, Schmid $\mathrm{R}^{2}$, Munkhuu $\mathrm{B}^{3}$, Baumann $\mathrm{T}^{1}$
}

Institut 1 Institut für Hausarztmedizin und Community Care, Luzern/CH;

2 Baarer Kinderarztpraxis, Baar/CH; 3 National Center for Maternal and Child Health, Ulaanbaatar/MN

DOI 10.1055/s-0037-1606954

Problemstellung Für eine Klassifizierung der Säuglingshüfte mittels Ultraschall sind Massnahmen zur Qualitätssicherung notwendig. In der Mongolei wurde ein nationales, Ultraschall-basiertes Screeningprogramm für Hüftdysplasie (DDH) eingeführt. Dieses multizentrische Vorhaben verlangt nach einem Qualitätskonzept, welches kontinuierlich und zuverlässig die Ultraschalldiagnostik überprüft und die Möglichkeit bietet korrigierend einzugreifen.

Patienten und Methode Rund 40.000 Neugeborene werden in der Mongolei pro Jahr im Rahmen des SwissMongolian Pediatric Projects mittels Ultraschall für DDH gescreent. Zur Überprüfung der Qualität der je vier annotierten Bilder (Hüftseite, Alter, Geschlecht) werden dabei zwei Methoden entwickelt. Erstens erlaubt ein webbasiertes, passwortgeschütztes Qualitätstool („HipScreen“) den Upload der vom Ultraschallgerät exportierten DICOM-Dateien durch den Screener. Vor Ort trainierte Experten und Schweizer Supervisoren können so alle Untersuchungen überprüfen und mit Kommentaren an den Screener zurücksenden. Das Tool wird den Mongolischen Partnern kostenlos zur Verfügung gestellt. Zweitens erarbeiten wir eine vollständig automatisierte Qualitätskontrolle mittels maschinellem Lernen durch ein feedforward künstliches neuronales Netz. Ein Algorithmus soll dafür in der Lage sein, anatomische Strukturen auf den Bildern zu erkennen und den Alpha-Winkel nach Graf zu überprüfen.

Ergebnis Allein in den letzten sechs Monaten wurden 19.664 Ultraschalluntersuchungen von Säuglingshüften durch Screener an 25 Abteilungen auf die Webplattform HipScreen geladen. Davon wurden über $90 \%$ durch einen Experten kontrolliert (siehe Bildschirmfoto: Kontrollstatus „closed by expert“). Das automatisierte Auswertungstool befindet sich noch in präklinischer Entwicklung.

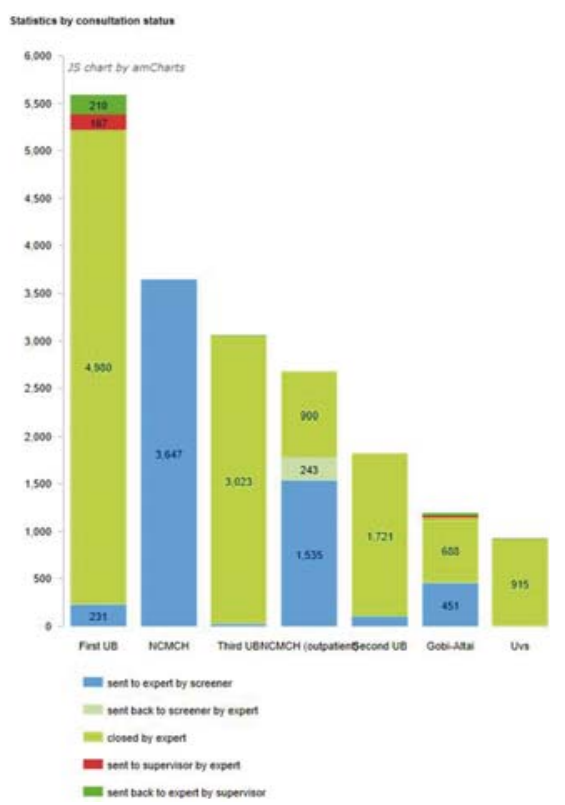

Abb. 1 Bildschirmfoto: Anzahl Hüftultraschall-Untersuchungen, eingeteilt nach Kontrollstatus (7 von 25 Abteilungen sind abgebildet) 
Schlussfolgerung Es entstehen neue, internetbasierte Konzepte, um eine qualitativ hochwertige Ultraschalldiagnostik der Säuglingshüfte sicherzustellen. Insbesondere in Weltregionen, wo Wissen und Ressourcen fehlen, sind diese für einen populationsweiten, effizienten Ultraschall notwendig. Aber auch in der Schweiz ist ein ähnliches Konzept in Vorbereitung.

\section{V2.003 Exposimetrie-Untersuchungen bei routinemäßigen Ultraschall-Anwendungen}

Autoren Malek $\mathrm{M}^{1}$, Kollmann $\mathrm{C}^{1}$

Institut 1 Zentrum für Medizinische Physik und Biomedizinische Technik,

Wien/AT

DOI 10.1055/s-0037-1606955

Problemstellung Während Ultraschalluntersuchungen kommt es zu einer Interaktion zwischen Ultraschallwellen und menschlichem Gewebe. Das hiermit entstehende Risiko möglicher auftretender Bioeffekte wird mit dem thermischen und mechanischen Index (TI, MI) abgeschätzt. Da dieses Wissen jedoch nicht bei allen durchführenden Untersuchern vorhanden ist werden diese oft nicht beachtet. Nun stellt sich die Frage nach der tatsächlichen Exposition der Patienten in der Gegenwart, aber auch der Entwicklung aus einer identischen Erhebung 2010.

Patienten und Methode Der TI und MI wurden mit dazugehörigen Rahmenbedingungen (Gerät, Schallkopf, Modus, Programm) im zeitlichen Verlauf einer kompletten Untersuchung dokumentiert. Die teilnehmenden Patienten eines mitwirkenden Universitätsspitals mit unterschiedlichen Kliniken (Kinderradiologie, Kardiologie, Frauenheilkunde, Gastroenterologie und Hepatologie, Radiologie) wurden per Zufall ausgewählt. Insgesamt 320 Untersuchungen wurden dokumentiert und in folgende vier Gruppen eingeteilt - Pädiatrie (99), Kardiologie (79), Gynäkologie (50), Abdominelle Untersuchungen (92). Die Grundlagen zu Aussagen über Maxima („max“), Grundeinstellungen („boot“) der Geräte/Programme und Verlauf vollständiger Untersuchungen, sind somit gegeben.

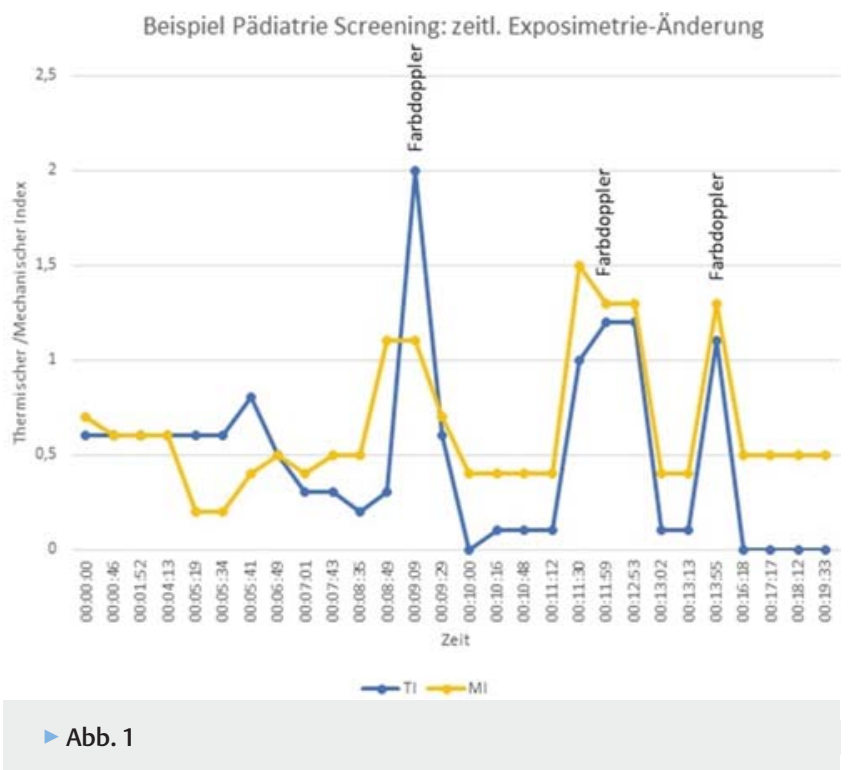

Ergebnis Der Vergleich zwischen aktuellen Daten und Daten aus 2010 lässt einen signifikanten Unterschied im Sinne einer Elevation in allen errechneten Merkmalen ( $\mathrm{TI}_{\max }, \mathrm{MI}_{\max }, \mathrm{TI}_{\text {boot }}, \mathrm{MI}_{\text {boot }}$ ) in den Gruppen Pädiatrie und Gynäkologie darstellen. In der Gruppe Abdomen konnten keine signifikaten Unterschiede berechnet werden. Eine signifikante Senkung aller Merkmale bis auf $\mathrm{TI}_{\max }$ konnte in der Kardiologie festgestellt werden.

Schlussfolgerung Ein Output mit steigender Tendenz in den letzten Jahren kann angenommen werden. Eine Ausnahme bildet hier das Fach Kardiologie.
Außerdem konnte der Einfluss unterschiedlicher Parameter auf den TI und MI aufgezeigt werden.

\section{V2.004 Schädelsonografie für alle Neugeborenen? Daten aus der populationsbasierten SNiP-Studie}

Autoren Lange $\mathrm{A}^{1}$, Weise $\mathrm{J}^{2}$, Bahlmann $\mathrm{H}^{2}$, Ittermann $\mathrm{T}^{1}$, Heckmann $\mathrm{M}^{2}$ Institut 1 Universitätsmedizin, Greifswald/DE; 2 Universitätsmedizin Greifswald, Greifswald/DE DOI 10.1055/s-0037-1606956

Problemstellung Eine Schädelsonografie (SSG) wird bei Neugeborenen (NG) $<30$ Schwangerschaftswochen (SSW) und nach Indikation durchgeführt. Ein SSG-Screening aller NG gibt es nicht. Ist die SSG bei allen NG eine sinnvolle Screening-Maßnahme?

Patienten und Methode 5109 NG erhielten im Rahmen der SNiP Studie (Survey of Neonates in Pomerania) eine SSG. Es wurden drei Gruppen gebildet: (I) NG mit Indikation für die SSG, (Ila) NG ohne Indikation aber mit stationärer Aufnahme, (IIb) NG ohne Indikation und ohne stationäre Aufnahme. Die Indikationen wurden den „AIUM Practice Guidelines for Neurosonography 2014“ entnommen. Alle auffälligen Befunde wurden von einer Neonatologin (AL) nachbefundet, eine Einteilung in vorab definierte milde (z. B. IVH I ${ }^{\circ}-I^{\circ}$, unilaterale/singuläre Zysten, Ventrikelasymmetrien) und schwere Auffälligkeiten (z.B. IVH III-IVº Corpus-callosum-Fehlbildungen, Hydrocephalus, Hirnödem, bilaterale/multiple Zysten) vorgenommen und das neurologische Follow-Up dokumentiert.

Ergebnis Von 5109 untersuchten NG fanden sich 224/5064 NG $\geq 30$ SSW mit auffälliger SSG (4,4\%): Gruppe I $(n=747) 44$ milde und 10 schwere Auffälligkeiten; Gruppe Ila ( $n=621) 36$ milde und 17 schwere; Gruppe IIb ( $n=3696$ ) 103 milde und 14 schwere Befunde ( $n=12$ bilaterale und/oder multiple Zysten, $n=1$ PVL, $n=1$ Corpus callosum-Hypoplasie). Neurologische Entwicklungsdefizite in Ilb entwickelten sich bei $14 \mathrm{NG}$ mit milder Störung und 1 NG mit schwerer Auffälligkeit.

Schlussfolgerung Auch bei NG ohne Indikation für eine Schädelsonografie fanden sich klinisch relevante Auffälligkeiten. Die kumulative Inzidenz betrug $2,8 \%$ bzw. $0,38 \%$ für IIb. Es fand sich bei $12,8 \%$ in Ilb ein auffälliges neurologisches Follow Up (I: 20,4\%; Ila: 18,9\%). Ob diese neurologischen Störungen jedoch einen günstigeren Verlauf durch die frühe SSG genommen haben, bedarf weiterer Analysen.

\section{V2.005 Thoraxsonografie in der Diagnostik atypischer Pneumonien im Kindesalter}

Autoren Sengewein j ${ }^{1}$, Rosenbaum $\mathrm{T}^{1}$, Feldkamp $\mathrm{A}^{1}$

Institut 1 Sana Kliniken Duisburg, Duisburg/DE

DOI 10.1055/s-0037-1606957

Problemstellung Pneumonien sind eine der häufigsten infektiologischen Ursachen für stationäre Krankenhausaufenthalte im Kindesalter. Neben Auskultationsbefund und Laborparametern ist die Röntgenaufnahme der Goldstandard in der Diagnostik. Oft lässt sich jedoch kein eindeutiger Befund erheben. Dank hochauflösender Schallköpfe nimmt die Lungensonografie in den letzten Jahren einen wachsenden Stellenwert ein. Zeichen einer bakteriellen (typischen) Pneumonie sind flächenhafte Belüftungsstörungen. Bei interstitiellen (atypischen) Pneumonien finden sich Pleuraaufrauhungen, kleine subpleurale Herde, ein eingeschränktes Pleuragleiten, vermehrte B-Linien und minimale Pleuraergüsse.

Patienten und Methode Es wurden 24 Patienten im Alter von 1 Monat bis 17 Jahren mit klinischem Verdacht einer Pneumonie ausgewertet, bei denen im Röntgen keine sicheren Pneumoniezeichen zu erkennen waren. Die Beurteilung der Röntgenbilder wurde von einem kinderradiologisch versierten Arzt vorgenommen. Alle Patienten erhielten zur weiteren Diagnostik eine Lungensonografie vom jeweils gleichen Untersucher. Zusätzlich wurden ein großes Blutbild und CRP, sowie eine Mykoplasmenserologie und ein respiratorisches Panel bestimmt. 
Ergebnis In allen Fällen zeigten sich sonografisch spezifische Zeichen für eine interstitielle Belüftungsstörung. Durchschnittlicher CRP-Wert 3,9 mg/dl. Durchschnittlicher Leukozyten-Wert 13,1/nl. Anzahl positiver Mykoplasmenserologien 13/24 Patienten. Anzahl positiver Virusnachweise 20/24 Patienten. Schlussfolgerung Eine Lungensonografie kann in der Diagnostik einer Pneumonie, gerade bei unklareren Fällen, hilfreiche Zusatzinformationen liefern. Interstitielle Belüftungsstörungen lassen sich per Sonografie besser differenzieren als radiologisch. Bei eindeutigem sonografischen Befund verzichteten wir auf eine antibiotische Therapie. Ob hierdurch sogar auf eine Röntgenuntersuchung verzichtet werden kann, kann anhand dieser Daten nicht beantwortet werden, sollte aber individuell erwogen werden.

\section{V2.006 Netzwerk Fetalkardiologie Hannover: Klinisches Outcome von Neugeborenen mit pränatal diagnostizierten Herzfehlern - unter Berücksichtigung der Reproduzierbarkeit der prä- und postnatal erhobenen Ultraschallbefunde}

Autoren Schubert $C^{1}$, Kaisenberg $C$ von $^{1}$, Staboulidou $I^{1}$, Schild $R^{2}$, Peter $D^{1}$, Bohnhorst $B^{1}$, Wüstemann $M^{3}$, Schwerdtfeger $R^{3}$, Mühlhaus $K^{3}$, Koluch $A^{4}$, Beerbaum $\mathrm{P}^{1}$

Institut 1 Medizinische Hochschule Hannover, Hannover/DE; 2 Diakovere

Perinatalzentrum Hannover, Hannover/DE; 3 Zentrum für Pränatalmedizin,

Hannover/DE; 4 Kinderkrankenhaus auf der Bult, Hannover/DE

DOI 10.1055/s-0037-1606958

Problemstellung Herzfehler gehören zu den häufigsten angeborenen Fehlbildungen. Das Spektrum dieser Vitien reicht von einfachen Anomalien bis zu komplexen Anatomien. Das klinische Outcome dieser Kinder ist entscheidend von der pränatalen Ultraschalldiagnose abhängig. Kinder mit komplexen, vorgeburtlich unbekannten Herzfehlern entwickeln gehäuft lebensbedrohliche Kreislaufdekompensationen und Komorbiditäten.

Patienten und Methode Offene prospektive Registerstudie: im interdisziplinären Setting erfolgten 270 Fetalkardiologische-Konsile bei etwa 150 Feten mit angeborenen Herzfehler zwischen April 2014 und März 2017. Die Eltern wurden aus kinderkardiologischer Sicht über den Herzfehler klinisch eingehend beraten. Nach Geburt erfolgten die weitere klinische Evaluation des Vitiums und die Einleitung notwendiger Therapieschritte. Insgesamt werden etwa 120 Neonaten mit einem Vitium jährlich in der Abteilung für Pädiatrischen Kardiologie, $\mathrm{MHH}$ zumeist operativ oder interventionell behandelt.

Ergebnis Bei etwa 25-30\% der lebendgeborenen Neonaten mit Herzfehler ist das Vitium pränatal bekannt. In >95\% der Fälle konnte die vorgeburtlich mittels Ultraschall gestellte Diagnose postnatal bestätigt werden. Die Rate der komplexen Vitien (>1 kardialer Defekt) betrug ca. 65\%. Aufgrund des bereits bekannten Vitiums ist eine an die Komplexität der Hämodynamik angepasste Therapie unmittelbar umsetzbar gewesen. Dabei konnte eine Senkung der Mortalität auf $<3 \%$ erzielt werden bei erwarteter Mortalitätsrate $>15 \%$ nach Lage der Literatur bei pränatal nicht-diagnostizierten Herzfehlern.

Schlussfolgerung Die Gesamtdetektionsrate der Herzfehler ist noch ungünstig (<30\%), allerdings ist eine hohe diagnostische Qualität bei den überwiesenen Feten zu verzeichnen. Im Vergleich zu Kontrollen ohne pränatale Herzfehleridentifizierung deutlich geringere Gesamtmortalität. Mithilfe der Ultraschalldiagnostik lassen sich Herzfehler bereits in einer frühen Schwangerschaftswoche zuverlässig diagnostizieren. Im Rahmen einer interdisziplinären Beratung und Betreuung lassen sich die Therapieaussichten erheblich verbessern.

\section{V2.007 Die Elastografie der Schilddrüse bei Kindern und Jugendlichen mittels Acoustic Radiation Force Impulse Imaging - Aussagekraft, Geschlechtsunterschiede und Normwerte}

\author{
Autoren Nurkan $E^{1}$, Behrens $\mathrm{CB}^{1}$, Harth $\mathrm{S}^{1}$, Krombach $\mathrm{GA}^{1}$ \\ Institut 1 Universitätsklinikum Gießen, Gießen/DE \\ DOI 10.1055/s-0037-1606959
}

Problemstellung Im pädiatrischen Bereich gibt es bislang nur wenige Ergebnisse zur ARFI-Elastografie der Schilddrüse. Ziel dieser prospektiven Studie waren die Ermittlung von Normwerten sowie die Untersuchung auf mögliche geschlechtsspezifische Unterschiede und des Streuungsverhaltens.

Patienten und Methode Insgesamt wurden 71 ARFI-Elastografien der Schilddrüse an 38 Gesunden und 33 Patienten mit Hashimoto-Thyreoiditis von 0 bis 18 Lebensjahren durchgeführt. Die Elastografiewerte wurden hinsichtlich ihrer Schwankungen mittels Variations- und Intraklassen-Korrelations-Koeffizient überprüft und auf Unterschiede zwischen beiden Gruppen sowie zwischen den Geschlechtern untersucht. Zur Ermittlung des Cut-off-Wertes wurden ROC-Analysen und der Youden's Index angewendet.

Ergebnis Die ARFI-Elastografie der Schilddrüse zeigte in allen Gruppen nur geringe Streuungen. Die Mädchen wiesen in beiden Gruppen höhrere Werte auf (weiblich: gesund $1,36 \mathrm{~m} / \mathrm{s}(0,23 \mathrm{SD})$, Hashimoto $1,75 \mathrm{~m} / \mathrm{s}(0,28 \mathrm{SD})$ gegenüber männlich: gesund $1,16 \mathrm{~m} / \mathrm{s}(0,17 \mathrm{SD})$, Hashimoto $1,71 \mathrm{~m} / \mathrm{s}(0,19$ $\mathrm{SD})$ ). Ein signifikanter Zusammenhang zwischen Probandenalter und Elastografie lag nicht vor. Im Rahmen der ROC-Analyse zeigte sich eine gute Trennung der beiden Gruppen (AUC (area under the curve): 0,93). Zur Bestimmung des Cutoff-Wertes kam es auf eine dem Untersuchungszweck angepasste Balance zwischen Sensitivität $(0,85)$ und Spezifität $(0,87)$ an, die bei einem Cutoff-Wert von $1,49 \mathrm{~m} / \mathrm{s}$ erfüllt ist.

Schlussfolgerung Schwankungen der Elastografiemesswerte waren hauptsächlich auf interindividuelle Unterschiede zurückzuführen. Für die Trennung beider Gruppen kann ein Cutoff-Wert von 1,49 m/s herangezogen werden. Hinsichtlich der höheren Messwerte der Mädchen könnten für eine zusätzliche Verbesserung von Sensitivität und Spezifität geschlechtergetrennte Normwerte in Erwägung gezogen werden.

\section{9: Pädiatrie II}

\section{F 5 Bilateraler Lungensequester - eine seltene postnatale Diagnose}

Autoren Wowra $\mathrm{T}^{1}$, Scheidt $\mathrm{F}$ von ${ }^{1}$, Brunner $\mathrm{H}^{1}$, Hummler $\mathrm{H}^{1}$, Apitz $\mathrm{C}^{1}$ Institut 1 Universitätsklinikum Ulm, Ulm/DE

DOI 10.1055/s-0037-1606960

Problemstellung Der sonomorphologische Befund eines Lungensequesters ist bestens bekannt. Seine bilaterale Erscheinungsform stellt jedoch eher eine Seltenheit dar. Differentialdiagnostisch gilt es ihn insbesondere von einer CCAM (Congenitale Cystische Adenomatoide Malformation) abzugrenzen. Patienten und Methode Im vorliegenden Fall eines Frühgeborenen der 30. Schwangerschaftswoche (1250 g) war der Befund pränatal nicht bekannt. Es zeigten sich am 7. Lebenstag folgende sonografische Charakteristika: ein kaliberstarkes, akzessorisches Gefäß in der farbkodierten Duplexsonografie aus der Aorta oberhalb des Truncus coeliacus (TC), welches nach einer Aufzweigung in eine schmetterlingsförmige, echoreiche, scharf abgrenzbare Struktur beidseits im unteren Thorax mündete (Abb. 1 und 2).

Eine Magnetresonanztomografie bestätigte wenige Wochen später den sonografischen Befund und zeigte den venösen Abfluss über die Pulmonalvenen. 


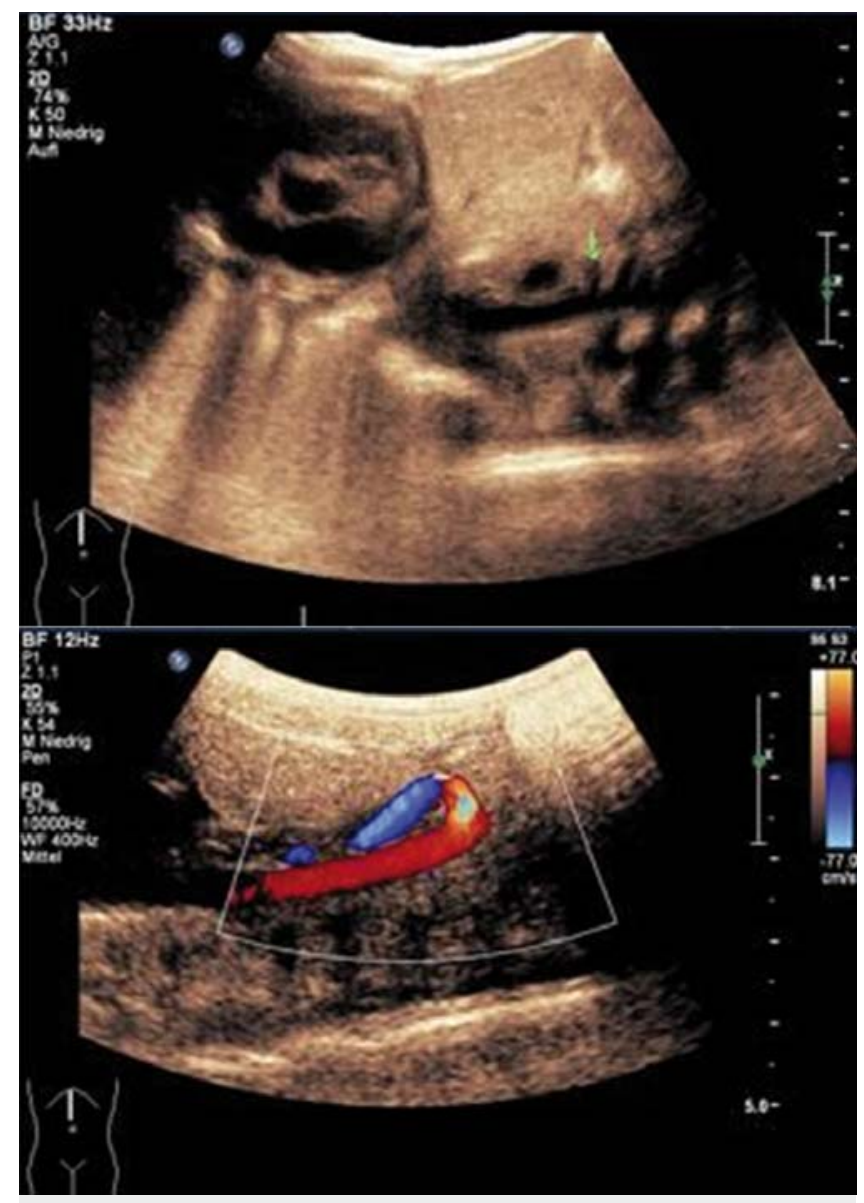

D Abb. 1 Sequesterarterie - akzessorisches Gefäß cranial des TC

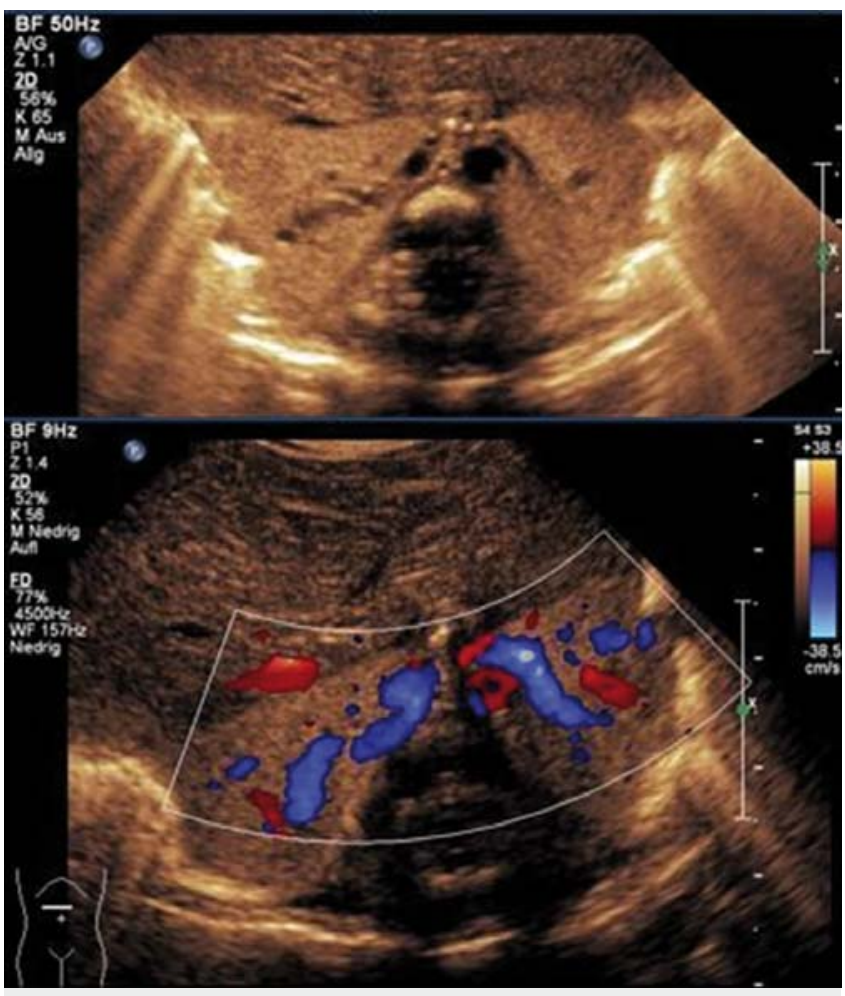

- Abb. 2 Sequester - bilaterale, schmetterlingsförmige, echoreiche, homogene Masse mit kräftiger Perfusion
Ergebnis Als Zeichen des ausgeprägten Links-Links-Shunts konnte in den sonograpischen Kontrolluntersuchungen eine linksseitige Vorhofdilatation beobachtet werden (Abb. 3).

Vorübergehend pathologische Flussprofile im Sinne eines Steel-Phänomens zeigten sich in den Arterien distal des aberranten Gefäßes (Abb. 3).

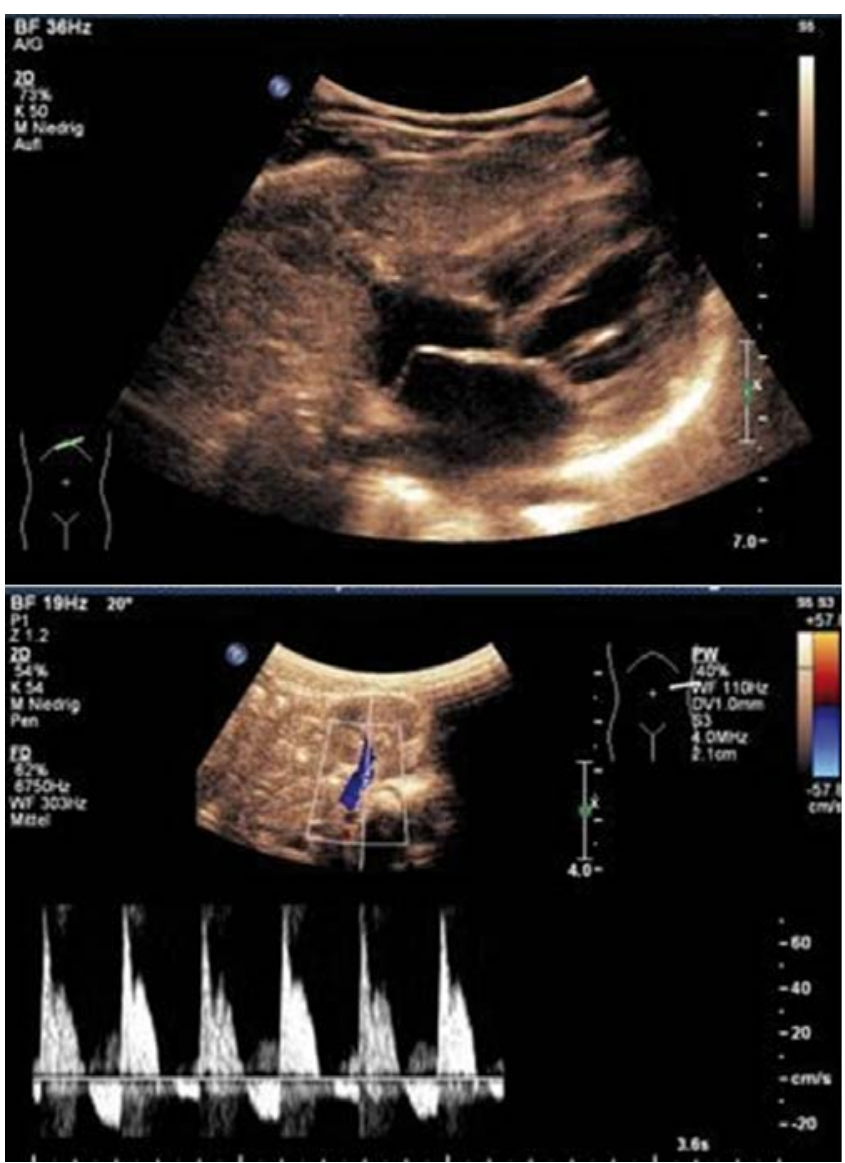

Abb. 1 Linksseitige Vorhofdilatation und pathologischer Fluss in der A. renalis

Schlussfolgerung Aufgrund der stabilen kardiorespiratorischen Situation konnte unter engmaschigen sonografischen Kontrollen der optimale Zeitpunkt für eine endovaskuläre Embolisation der Sequesterarterie abgewartet werden.

F 6 Sonografie und Kontrastmittelsonografie (CEUS) bei einem Kleinkind mit nodulärer regenerativer Hyperplasie (NRH) der Leber bei Tyrosinämie Typ 1

Autoren Taut $\mathrm{H}^{1}$, Hahn $\mathrm{G}^{2}$

Institut 1 Medizinische Fakultät der TU Dresden, Klinik und Poliklinik für Kinder- und Jugendmedizin, Dresden/DE; 2 Medizinische Fakultät der TU Dresden, Institut und Poliklinik für radiologische Diagnostik, Dresden/DE DOI 10.1055/s-0037-1606961

Patienten und Methode Vorgestellt wird ein 12 Monate altes Mädchen mit einer Tyrosinämie Typ 1. Dabei handelt es sich eine autosomal rezessive Störung im Stoffwechsel der Aminosäure Tyrosin mit Symptomen an Leber, Nieren und dem peripheren Nervensystem. Die Prävalenz beträgt 1:100.000. Die Sonografie zeigte eine mäßige Hepatomegalie mit diffus kleinknotiger Texturveränderung und multiplen sowohl echoreichen als auch echoarmen Noduli bis zu $11 \mathrm{~mm}$ Durchmesser. Duplexsonografisch bestand noch keine portale Hypertension, elastografisch fanden sich neben Arealen regelrechter 
Elastizität Areale mit hoher Steifigkeit. Morphologisch war ein Hepatozelluläres Karzinom nicht auszuschließen. Dieses bestätigte sich in der Kontrastmittelsonografie und im MRT sowie paraklinisch ( $\alpha$-Fetoprotein) nicht. Es handelte sich in diesem Stadium der Erkrankung um eine noduläre regenerative Hyperplasie. Diese ist charakterisiert durch Atrophie des Lebergewebes ohne Fibrose und regenerativen Knötchen, welche meist echoarm, aber auch hyperechogen sein können.

Schlussfolgerung Bei der Tyrosinämie Typ 1 besteht ein hohes Risiko der Entstehung eines Hepatozellulären Karzinoms. Deshalb sind regelmäßige sonografische Kontrollen unabdingbar. Hier spielt neben der gesamten Palette sonografischer Diagnostik, wie B-Bild, Duplexsonogaphie und Elastografie die Kontrastmittelsonografie auch im jungen Kindesalter eine zunehmend wichtigere Rolle. Daneben sind die regelmäßige Bestimmung des $\alpha$-Fetoproteins und bei sonografisch verdächtigem Befund MRT-Untersuchungen indiziert.

\section{F 7 Kortikale Nekrosen unklarer Genese bei asymptomatischem Frühgeborenen}

\section{Autoren Thomas $\mathrm{V}$}

Institut 1 Klinikum Dritter Orden, München/DE

DOI 10.1055/s-0037-1606962

Patienten und Methode Bei unserem Patienten handelt es sich um ein hypotrophes Frühgeborenes von 28+4 SSW, GG $790 \mathrm{~g}$. Indikation zur Sectio: fehlendes Wachstum, reduzierte Kindsbewegungen, HELLP-Symptomatik der Mutter.

Der Junge wurde bei der Erstversorgung im Kreißsaal intubiert, erhielt Surfactant, wurde nach wenigen Stunden extubiert und war im folgenden unter CPAP-Atemhilfe kardiorespiratorisch stabil. Ein Routinelabor im Alter von 36 Lebensstunden erbrachte ein erhöhtes $\operatorname{CrP}(2,96 \mathrm{mg} / \mathrm{dl})$ bei asymptomatischem Patienten, es wurde 5 Tage lang nach Standardschema kalkuliert antibiotisch behandelt, die Blutkultur war negativ.

Routinemäßig durchgeführte Schädelsonografien waren im Alter von 1, 4, 12 und 25 Lebenstagen unauffällig. Am Tag 40 bot sich ein stark verändertes Bild: Es zeigten sich beidseits periventrikulär multiple porenzephale Zysten, kortikale und subkortikale Läsionen parietal, parietooccipital und temporal. Das Verteilungsmuster ließ nicht auf ein ischämisches Geschehen schließen, die infektiöse Genese war mit dem klinischen Verlauf schlecht vereinbar. MRtomografisch erhärtete sich die Vermutung einer Nekrosebildung bei Cerebritis. Ein Keimnachweis gelang trotz ausführlicher Diagnostik (Blutkulturen, Lumbalpunktion, Punktat Hirnabszess mit Kultur und Virus-PCR) nicht. Es erfolgte eine antibiotische und antimykotische Therapie über insgesamt 15 Tage. Die zentralen Läsionen dehnten sich nicht weiter aus. Trotz der extensiven Nekroseherde entwickelt sich der Patient bislang (Alter 5 Monate) neurologisch unauffällig.

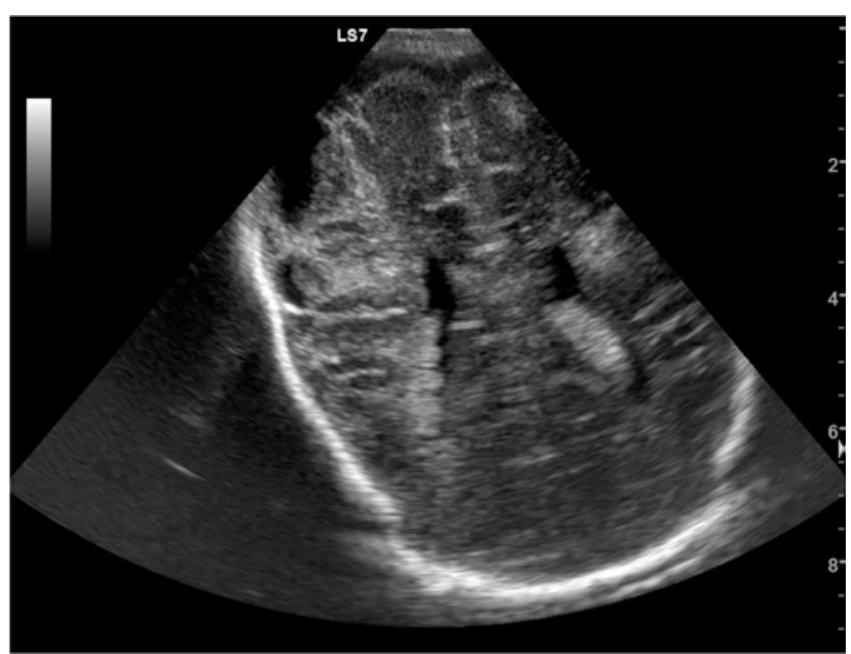

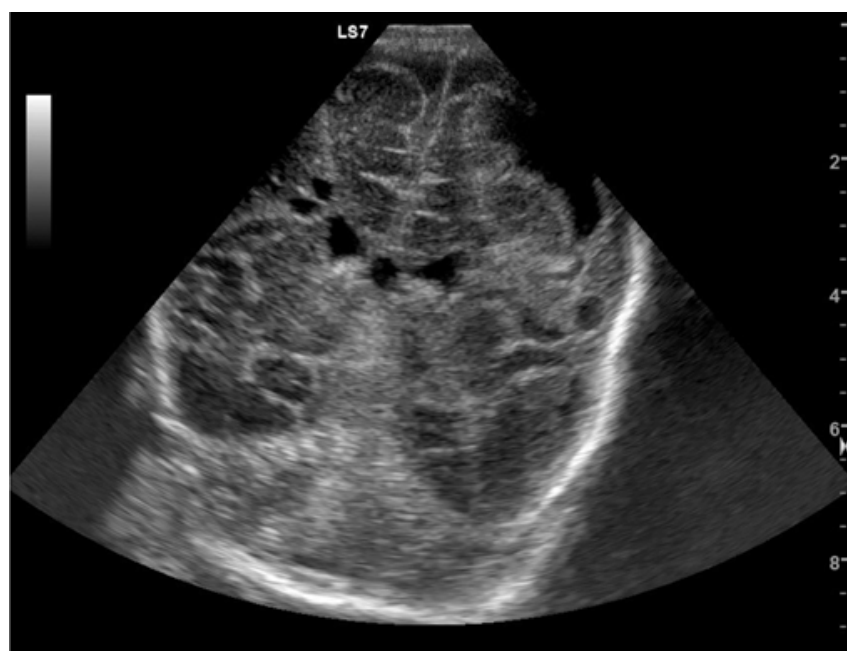

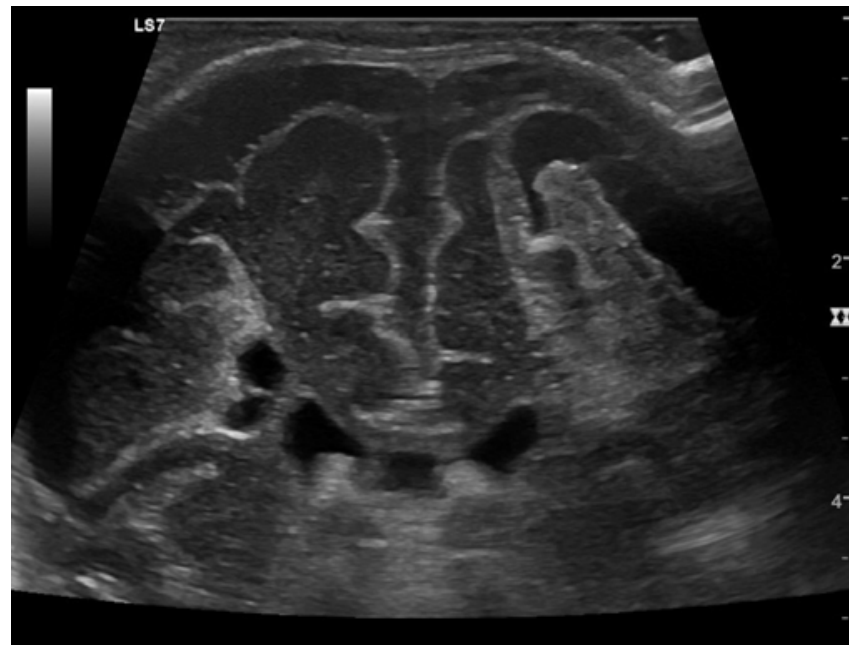

Schlussfolgerung Die Versorgung von extremen Frühgeborenen gehört zur alltäglichen Routine. Dabei dürfen auch bei scheinbar problemlosen Verläufen die Risiken der Frühgeburtlichkeit nicht unterschätzt werden. Hierzu gehören ungewöhnliche Infektionen und deren schwerwiegende Komplikationen.

\section{F 8 Akute Pankreatitis beim Kleinkind durch Dislokation der perkutanen Ernährungssonde}

Autoren Thomas $\mathrm{V}^{1}$

Institut 1 Klinikum Dritter Orden München, München/DE

DOI 10.1055/s-0037-1606963

Patienten und Methode Ein 1 10/12 J. alter, schwer psychomotorisch retardierter Junge wurde aufgrund von rezidivierendem Erbrechen und allgemeiner Zustandsverschlechterung vorgestellt. Vorbekannte Grunderkrankungen: Lissenzephalie - Tubulinopathie, epileptische Enzephalopathie und bilaterale dyskinetische Zerebralparese GMFCS Level V. Z.n. Anlage einer perkutanen Ernährungssonde (anamnestisch PEG) in externem Krankenhaus.

Das Aufnahmelabor wies auf eine Pankreatitis, die initiale Verdachtsdiagnose lautete Valproat-assoziierte Pankreatitis. Die Sonografie - erschwert durch die physischen Besonderheiten des Patienten - zeigte ein Subileusbild mit einem trotz nüchternem Kind extrem dilatierten Magen, einen ausgeprägten gastroösophagealen Reflux sowie leichte Pankreatitiszeichen. Ursächlich hierfür fand sich ein ungewöhlich stark dilatierter Blockungs-Ballon der perkutanen gastralen Sonde (Gastrotube) mit Fehllage im proximalen Duodenum.

Beim Entblocken der Sonde konnte festgestellt werden, dass der Patient über den falschen Schenkel der gastralen Sonde (Blockungsschenkel) Milchnahrung erhalten hatte. Nach Entblockung und korrekter Platzierung der Sonde nor- 
malisierten sich - unter antibiotischer und symptomatischer Therapie - Zustand und Laborwerte des kleinen Patienten rasch.

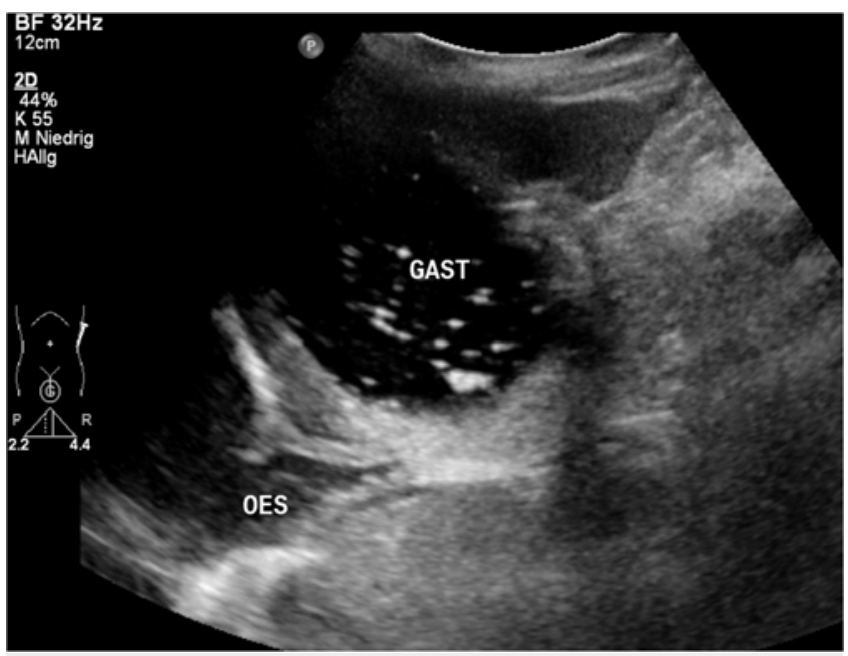

Abb. 1 dilatierter Magen, GÖR

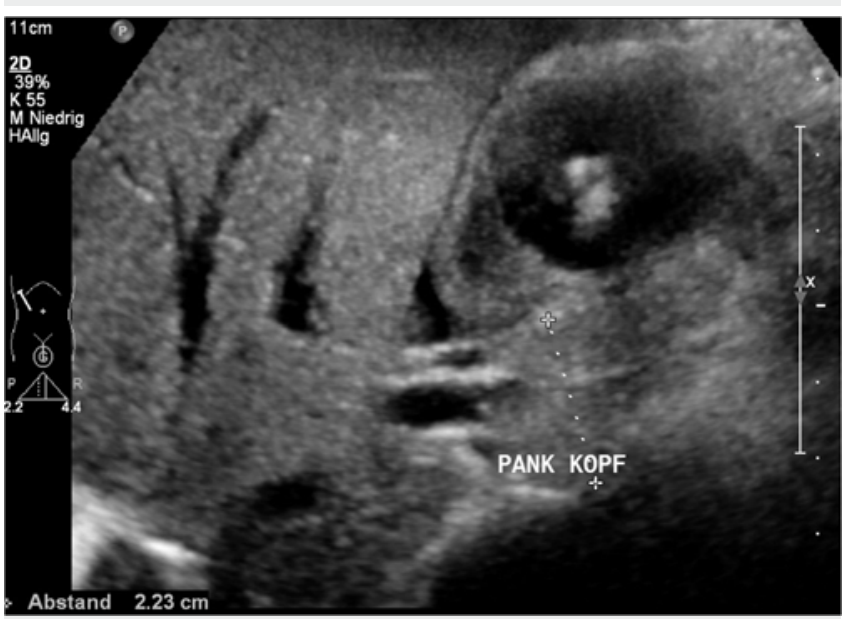

Abb. 2 Pankreaskopf, Gastrotube

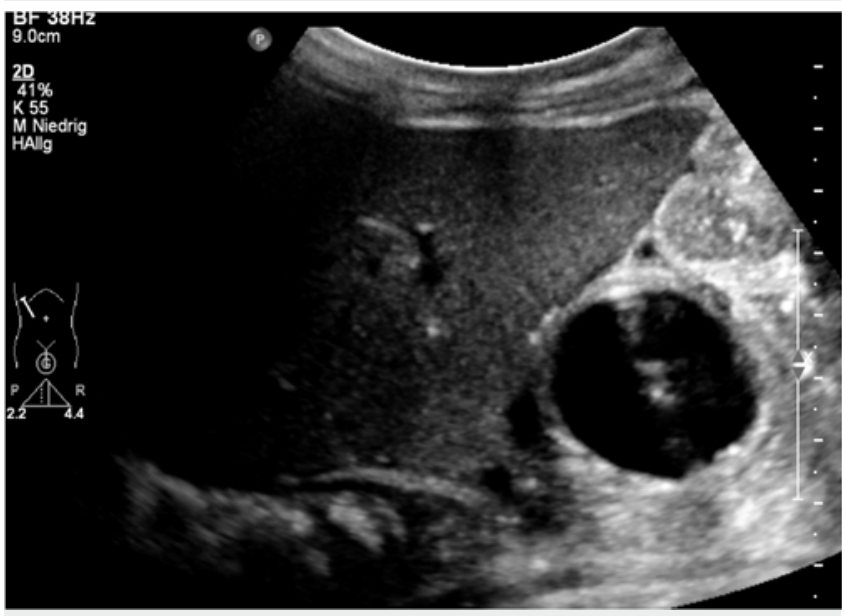

Abb. 3 Gastrotube-Ballon in prox. Duodenum

Schlussfolgerung Patienten mit schwerer Entwicklungsstörung stellen ein besonderes Patientengut dar. Die Diagnostik ist durch eine begrenzte Kommunikationsfähigkeit des Patienten erschwert, zudem bestehen bei den Patienten durch körperliche Besonderheiten oft eingeschränkte Schallbedingungen.
Dennoch und gerade hier ist der Ultraschall ein unverzichtbares Handwerkszeug.

Die akute Pankreatitis ist eine seltene, aber potentiell lebensbedrohliche Komplikation von gastralen, ballongeblockten Ernährungssonden. Die mechanische Obstruktion der Papille ist eine wichtige differentialdiagnostischen Überlegungen zur Ätiologie der akuten Pankreatitis. Die genaue Kenntnis der Patientenhistorie einschließlich Installationen sowie der Informationsfluss zwischen behandelnden Ärzten ist hierfür ein entscheidender Faktor.

\section{4: Technik}

\section{V4.001 Nicht-invasive präklinische Bildgebung auf Zellebene mittels Raster Scanning Optoacoustic Mesocopy}

Autoren Knieling $\mathrm{F}^{1}$, Claussen $\mathrm{J}^{2}$, Rascher $\mathrm{W}^{1}$, Neurath $\mathrm{M}^{1}$, Waldner $\mathrm{M}^{1}$ Institut 1 Universitätsklinikum Erlangen, Erlangen/DE; 2 iThera Medical $\mathrm{GmbH}$, München/DE

DOI 10.1055/s-0037-1606964

Problemstellung Optische Bildgebung ist ein wichtiges Werkzeug für Grundlagen- und präklinische Forschung, jedoch limitieren Lichtstreuungseffekte die Einsetzbarkeit auf Zellkulturen oder oberflächliche Gewebe. Die Raster Scanning Optoacoustic Mesocopy (RSOM) kombiniert 3D-Visualisierung bis zu $5 \mathrm{~mm}$ Eindringtiefe mit einer Auflösung bis $10 \mu \mathrm{m}$ und minimalen Akquisitionszeiten von 1,5-3 min. Ziel war es die Anwendbarkeit und Möglichkeiten von RSOM in präklinischen Modellen in- und ex-vivo zu zeigen.

Patienten und Methode Das RSOM (RSOM Explorer P50 iThera Medical $\mathrm{GmbH}$, München) induziert den optoakustischen Effekt durch kurze Bestrahlung mittels Laserlicht (532 nm) und Detektion von hochfrequenten Ultraschall (10-90Mhz) unter kontinuierliche Rasterung. Die entsprechenden Organe oder Versuchstiere wurden auf einer beheizten Untersuchungsliege platziert und narkotisiert. Als Modelle dienten gesunde C57BL/ 6 Mäuse und chemisch(DSS +/- AOM für Tumorentstehung, +/- transgen mit tdtomato-Expression in Fibroblasten) oder durch adoptiven T-Zelltransfer (CD $4^{+} C D 25^{\circ}$, labeling: CellTrace Yellow) induzierte Kolitismodelle. Gefäßfärbungen erfolgten durch systemische Perfusion mit 1,1'-dioctadecyl-3,3,3',3' tetramethylindocarbocyanine perchlorate (Dil).

Ergebnis Es konnte ex-vivo Mikro- und Markosvaskularisierung einzelner Organe 3D-hochauflösend darstellt werden; eine Färbung mittels Dil zeigte gegenüber dem Nativsignal des Hämoglobin eine weitere Verbesserung der bereits sehr guten Gefäßdarstellung (Abb. 1). In ex-vivo Kolitisexperimenten gelang sowohl die Darstellung von muskosaler Architektur und transgen-veränderten Zielzellen (Fibroblasten mit tdtomato) im gesunden als auch entzündeten und tumorösen Gewebe (Abb. 2). Weiterhin ließen sich im Transferkolitismodell auch einzelne gelabelte Zellen in der Darmschleimhaut nachweisen (Abb. 3). 

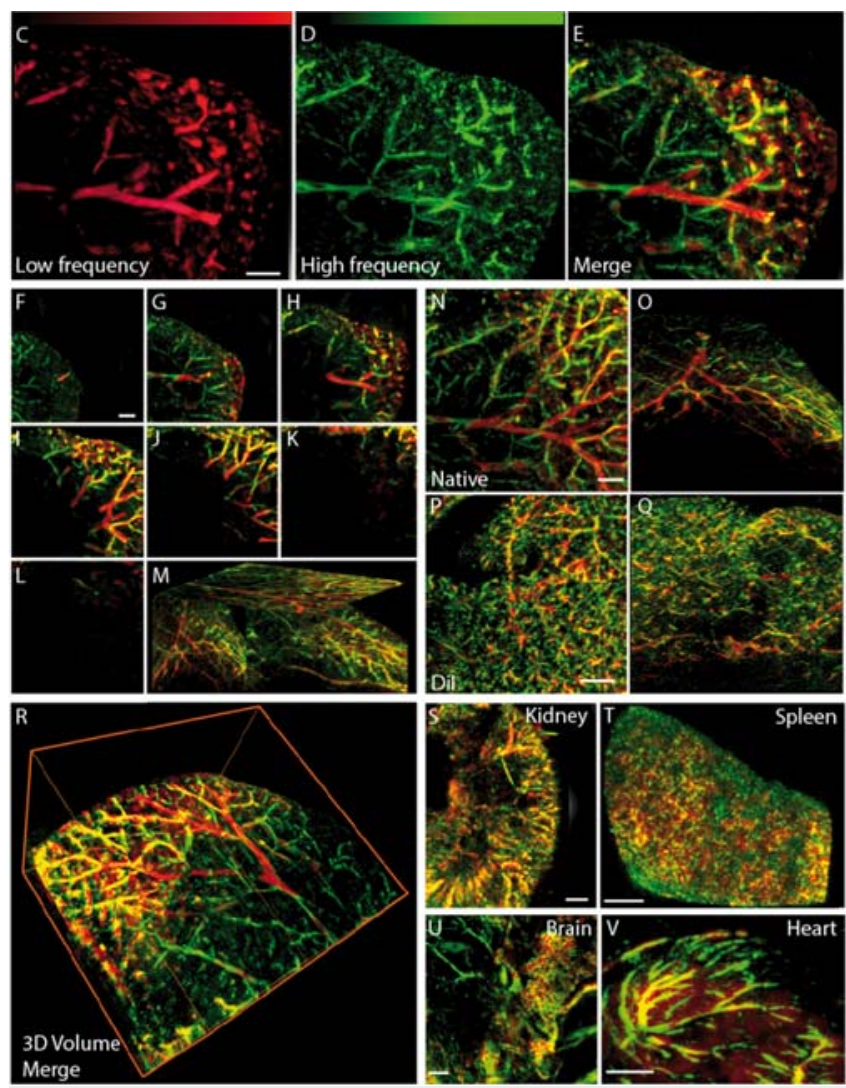

Abb. 1 Prinzip und ex-vivo Visualisierung von Organen. A: Setup, B: Optoakustisches Prinzip, C-E: Leber, F-L: XY alle 80 slices, M: alle 3 Schichten, N+O: Leber Nativ XY und YZ, P+Q: Leber Dil XY, R: 3DVolume, S: Niere, T: Milz, U: Gehirn, V: Herz

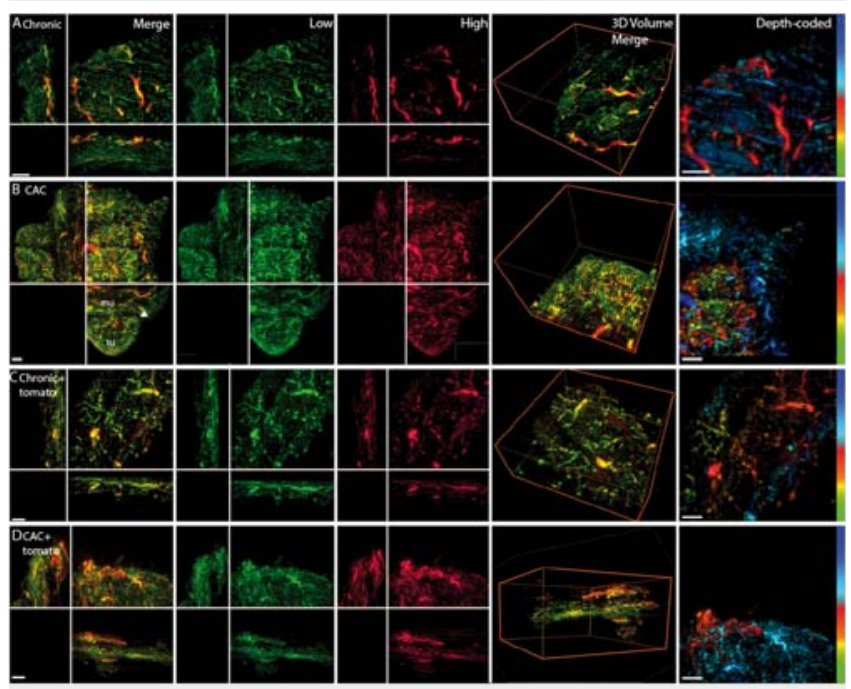

- Abb. 2 Ex-vivo Kolitis und Tumore A: Kontrolle in verschienden Ansichten B: Tumor in verschienden Ansichten (Dreiecke: Tumor, Pfeil: Gefäß) C: Fibroblasten mit tdtomato D: Fibroblast mit tdtomato und Tumor
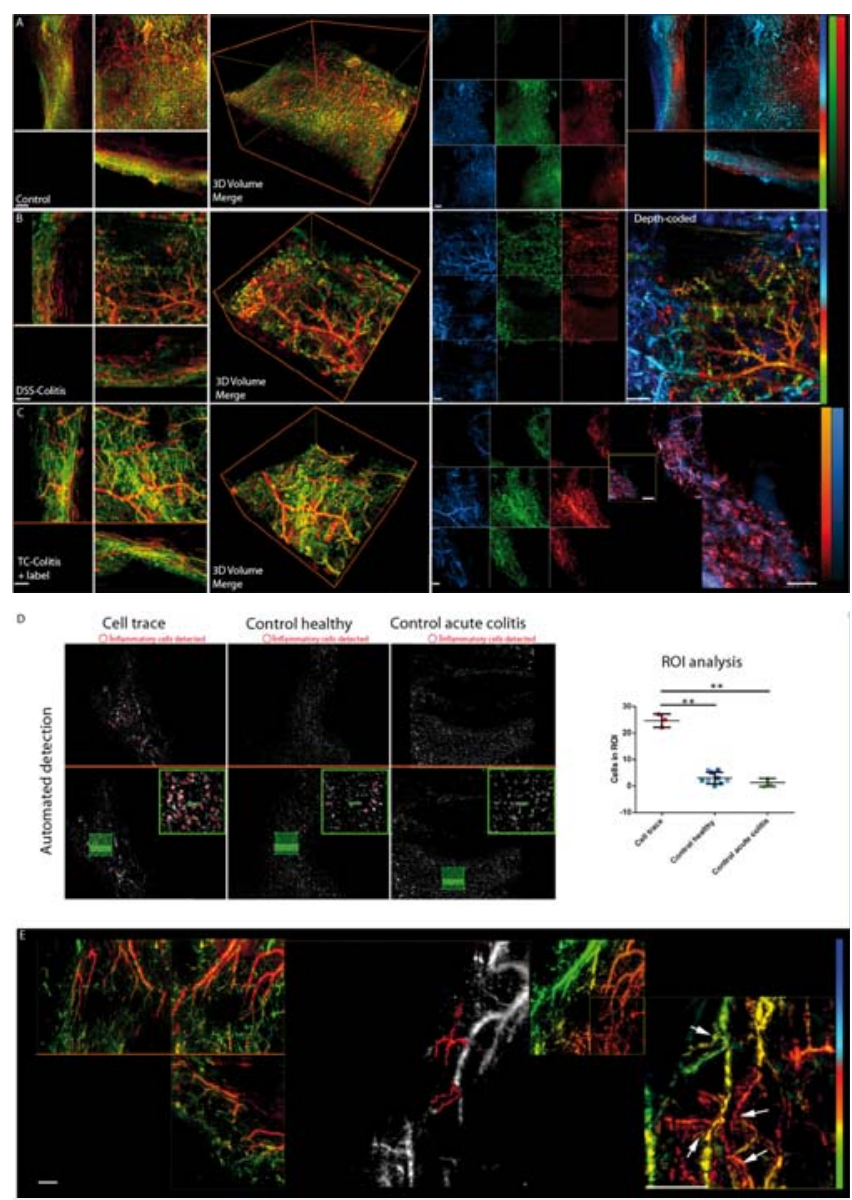

Abb. 3 In-vivo Kolitis A: Kontrolle: Blau: $10-30 \mathrm{MHz}$, Grün: 30 $90 \mathrm{MHz}$, Hot: $85-87 \mathrm{MHz}$ B: Akute Kolitis C: Transfer Kolitis D: Quantifizierung via Icy software (Scale1, 100, 1px, Size filter: $30-60)$ E: Fibroblasten mit tdtomato, Pfeile: Zellkörper

Schlussfolgerung RSOM ist eine neuartige Technologie zur Auflösung von Mirkostrukturen in präklinischen Modellen in-vivo. Tiefeneindringung, Auflösung und Scanzeiten bieten innovative neue Möglichkeiten für nicht-invasive Untersuchungen in murinen Modellen.

\section{V4.002 Lehrphantome zur Ultraschall- Artefaktdarstellungen unter Nutzung von Rapid-Prototyping Verfahren}

Autoren Kollmann $\mathrm{C}^{1}$, Hodul $\mathrm{A}^{1}$, Keilmann $\mathrm{N}^{1}$

Institut 1 Zentrum f. Med. Physik \& Biomed. Technik, Wien/AT DOI 10.1055/s-0037-1606965

Problemstellung Ein grundlegendes Verständnis der Ultraschallbildgebung und die Kenntnis der für die Modalität spezifischen auftretenden Bildartefakte ist essentiell, um eine hohe Diagnosequalität zu erreichen. Animationen und klinische Bilder kommen dabei meistens zum Einsatz. Spezielle Lehrphantome, die einzelne Artefakte einwandfrei produzieren können und so dem Anfänger im Skills-Lab eine individuelle Verständnisvermittlung ermöglichen, gab es bislang nicht. In dieser Arbeit werden Phantome vorgestellt, die die Wissensvermittlung gezielt und am Ultraschallgerät ermöglichen.

Patienten und Methode Das Phantomdesign ist so ausgelegt, dass es eine kompakte und vielfältige Nutzung zur Darstellung diverser Artefakttypen mittles 3D-Rapid Prototyping Drucks möglich wird. Die einzelnen Objekte sind in ein Material eingebettet, welches gewebe-dämpfende Eigenschaften aufweist und erst dadurch zu einigen Artefaktdarstellungen führt bzw. das charakteristische Ultraschall-Specklemuster erzeugt. 
Ergebnis Mit den bislang erstellten Phantomen können Schatten-, Verstärkungs-, Wiederholungs- und Spiegel-Artefakte eindeutig und wiederholbar dargestellt werden. Die einfache Handhabung gestattet es, die Phantome in Skills-labs individuell als Ergänzung zur Theorie zu nutzen und die reale Bildausprägung von Artefakten und zu erkennen bzw. auch Artefaktänderungen durch andere Geräteeinstellungen zu studieren.

Schlussfolgerung Die erstellten Lehrphantome sind erfolgreich in diversen Skills-Labs und Hand-on Kursen zum Einsatz gekommen und haben den Studierenden die spezielle Artefaktausprägung nach einer Theorieeinheit praktisch am Gerät vermittelt. Sowohl individuelles Erlernen als auch Erkennen (in einer Überprüfung) von speziellen Artefakten können diese Phantome unterstützen helfen.

\section{V4.003 Die Augmented Reality Ultraschall-App Studie (UppS)}

Autoren Lato $K^{1}$, Lato $C^{1}$, de Gregorio $N^{1}$, de Gregorio $A^{1}$, Polasik $A^{1}$, Schochter $F^{1}$, Friebe-Hoffmann $U^{1}$, Janni $W^{1}$, Ebner $F^{1}$

Institut 1 Klinik für Frauenheilkunde und Geburtshilfe, Ulm/DE

DOI 10.1055/s-0037-1606966

Problemstellung Bei der Ultraschallausbildung sind neben den theoretischen Kenntnissen die Entwicklung von motorischen Fähigkeiten entscheidend. Wir haben eine Augmented Reality Ultraschall-Anwendungssoftware entwickelt, um zusätzliche Übungen ohne Patient/-in zu ermöglichen. Im Rahmen der AppStudie wurde die Wertigkeit der neu entwickelten Apps getestet.

Patienten und Methode 33 Medizinstudenten haben die App getestet mit anschließend gegenseitiger Darstellung ihrer Nieren durch ein echtes Ultraschallgerät. Als Kriterien würden die dazu benötigte Arbeitszeit und die dargestellten Nierenparameter dokumentiert. 33 weitere Studenten haben als Kontrollgruppe nach schriftlicher Einführung und Erläuterung der Bedienung des Gerätes durch den Dozenten ebenso gegenseitig an sich den Nierenultraschall durchgeführt.

Ergebnis Es gab weder signifikante Unterschiede in den beiden Gruppen in Bezug auf die initialen Parameter (Alter, Geschlecht und Vorkenntnisse), noch gab es signifikante Unterschiede in der Arbeitszeit für die Nierendarstellung. In der App-Gruppe war die gemessene Niere signifikant größer (rechts: in der App-Gruppe: Median 105,3 mm (86,1 mm - 127,1 mm), Kontrollgruppe: Median 92 mm (50,4 mm - 112,2 mm); p<0,001, links: in der App-Gruppe Median 100,3 mm (81,7 mm - 118,6 mm), Kontrollgruppe: Median 85,3 mm $(48,3 \mathrm{~mm}-113,4 \mathrm{~mm}) ; p<0,001$. Dies weist darauf hin, dass in der AppGruppe die Nieren qualitativ besser erfasst wurden.

Schlussfolgerung Die neu entwickelte Augmented Reality Ultraschall-App scheint eine sinnvolle Ergänzung und Verbesserung beim Ultraschalltraining zu sein. Der neu entwickelte Algorithmus eröffnet den Weg zur Darstellung weiterer Organe und Pathologien.

\section{V4.004 Übersicht und neue Möglichkeiten der apparativen Qualitätssicherung bzw. Konstanzprüfung von Ultraschallgeräten und deren Sonden mit dem neuen TCC-3D-2.0 System}

Autoren Lange $\mathrm{L}^{1}$

Institut 1 Medizintechnik Lange, Marlow/DE

DOI 10.1055/s-0037-1606967

Problemstellung Die bisher am Markt erhältlichen Test- und Prüfsysteme lassen nur eine begrenzte Aussage über die Qualität und die Leistungsfähigkeit eines Ultraschalldiagnostiksystems zu.

Es bleiben leider einige Fragestellungen bei der Qualitäts- und Fehleranalyse offen.

Patienten und Methode Mit dem neuen TCC-3D-2.0 System (Fa. TCC, Timelkam, Austria) werden zusätzlich neue Parameter in der Qualitätsanalyse definiert.
1. Auswertung der Homogenität des Schallfeldes

2. Berechnung und Darstellung des akustischen Kontrastverhaltens des Ultraschallsystems

3. Darstellung des Schallfeldes des Ultraschallsystems (Haupt-, Neben- und Gitterkeulen), Berechnung der räumlichen Auflösung im Schallfeld

Der Test des Prüfsystems erfolgte unter realen Bedingungen des mobilen Serviceeinsatzes in Praxen und Kliniken.

Ergebnis Mit dem Prüfsystem lassen sich Qualitätsprobleme (geringe räumliche Auflösung, schlechtes Kontrastverhalten) erkennen und die möglichen Ursachen für bestehende Fehler und Defekte eingrenzen.

Eine schlechte Bildqualität mit einem geringen akustischen Kontrast hervorgerufen z. B. durch die Alterung von Sonden oder Sonden-Schäden sind nachweisbar.

Durch die qualitative und quantitative Darstellbarkeit des Schallfeldes ist eine Visualisierung der Hauptkeule sowie der Neben- und Gitterkeulen möglich.

Schlussfolgerung Das Test- und Prüfsystem ist für die Geräteprüfung von Ultraschallsystemen verschiedener Hersteller und Geräteklassen im Laborbetrieb und im mobilen Einsatz gut geeignet.

Bei entsprechender Festlegung von Grenzwerten ist die Verwendung des Systems im Rahmen der Technischen Qualitätssicherung und zur Konstanzprüfung von Ultraschallgeräten gut verwendbar.

\section{V4.005 Stellenwert der sonografisch gesteuerten Stanzbiopsie in der Diagnostik der ungeklärten Lymphadenopathie - eine retrospektive Studie bei $\mathrm{n}=793$ Patienten}

Autoren Wilczynski $A^{1}$, Trenker $C^{1}$, Görg $C^{1}$

Institut 1 Universitätsklinikum Marburg, Marburg/DE

DOI 10.1055/s-0037-1606968

Problemstellung Goldstandard in der Primärdiagnostik der unklaren Lymphadenopathie (LA) ist die komplette Lymphknoten (LK)-Exstirpation. Die sonografisch gesteuerte Stanzbiopsie bietet eine alternative Methode der Diagnosesicherung und wird zur Histologiegewinnung breitflächig eingesetzt. Patienten und Methode Im Zeitraum von 01/06-06/15 wurden $\mathrm{n}=793$ mit ungeklärter LA mit einer Vollschneid-Stanzbiopsienadel (BioPince) sonografisch gesteuert punktiert. Die LA war peripher (68\%) oder abdominell (32\%) gelegen. Die finalen Diagnosen waren: Nicht Hodgkin Lymphom (NHL) $n=245$, Hodgkin Lymphom $(H L) n=53$, Karzinommetastasen (MET) $n=356$, benigne LA $n=79$ und Sonstige $n=60$. Die Punktionsergebnisse wurden retrospektiv hinsichtlich Sensitivität (SE), Spezifität (SP) und Treffsicherheit (TR) untersucht.

Ergebnis Im Gesamtkollektiv aller $\mathrm{n}=793$ durchgeführten Biopsien betrug die SE 92,8\%, die SP 97,1\% und die diagnostische TR 93,6\%, wobei Subgruppen folgende Ergebnisse (SE, SP, TR) zeigten: $\mathrm{NHL}(93,8 \%, 99,8 \%, 98,0 \%), \mathrm{HL}$ (84,9\%, 100\%, 99,0\%), MET (96,5\%, 100\%, 97,4\%), benigne Erkrankungen (96,1\%, 99,7\%, 99,4\%) und Sonstige (96,6\%, 99,9\%, 99,6\%). In $n=90$ $(11,3 \%)$ Fällen wurde aus unterschiedlichen Gründen eine zusätzliche LK-Exstirpation durchgeführt. In n= 21 von 90 Fällen (23,3\%) kam es zu Abweichungen im Ergebnis der Stanzbiopsie und exstirpierten Lymphknotens ( $n=8 \mathrm{NHL}$, $\mathrm{n}=3 \mathrm{HL}, \mathrm{n}=6 \mathrm{MET}, \mathrm{n}=2$ Benigne, $\mathrm{n}=22$ Sonstige).

Schlussfolgerung Aufgrund der diagnostischen Treffsicherheit von 93,6\% im Gesamtkollektiv scheint die sonografisch gesteuerte Vollschneidstanzbiopsie bei ungeklärter LA eine alternative diagnostische Prozedur zur invasiven LK Exstirpation darzustellen. Eine prospektive vergleichende Studie zur definitiven Klärung des diagnostischen Stellenwertes der ultraschallgesteuerten Vollschneidebiospie in der Diagnostik ungeklärter LA ist notwendig. 


\section{P 1: Poster Abdomen \\ Poster Präsentation}

\section{P1.001 Liegt eine höhere Prävalenz von Krebs bei Patienten mit einem hepatischen Hämangiom vor?}

Autoren Akengin $A^{1}$, Malek NP², Boozari $B^{1}$, Kubicka $S^{1}$

Institut 1 Kreiskliniken Reutlingen, Reutlingen/DE; 2 Universitätsklinik

Tübingen, Tübingen/DE

DOI 10.1055/s-0037-1606969

Problemstellung Basiert auf einer klinischen Beobachtung haben wir uns die Frage gestellt, ob es eine höhere Prävalenz von Krebs bei Patienten mit einem hepatischen Hämangiom gibt.

Patienten und Methode Zwischen den Jahren 2012 und 2015 wurden 12056 Patienten in unserer Ultraschallabteilung untersucht. Bei 330 von den Untersuchten lag ein gesichertes hepatischen Hämangiom vor. Die Prävalenz von Krebs und das Risiko eine Krebserkrankung zu entwickeln wurde bei diesen Patienten retrospektiv untersucht und mit einer gleich großen Kontrollgruppe aus dem gleichen Patientenkollektiv (Geschlechtsverteilung und Alter waren zwischen den beiden Gruppen ausgewogen) verglichen.

Ergebnis Die Prävalenz von Krebs bei Patienten mit einem hepatischen Hämangiom war mit 28,5\% (95\% Cl; 23,7-33,7) signifikant höher als die der Kontrollgruppe mit 21,8\% (95\% Cl; 17,5-26,7), p<0,048. Das Risiko Krebs zu entwickeln war jedoch nicht unterschiedlich. Prävalenzratio (95\% Cl) Hämangiom versus Kontrolle betrug 1,31 (1,00 - 1,70), Odds Ratio (95\% Cl) Hämangiom versus Kontrolle lag bei 1,43 (1,00-2,03).

Schlussfolgerung Die Prävalenz von Krebs bei Patienten mit einem hepatischen Hämangiom war verglichen mit einem Kontrollkollektiv höher. Weitere prospektive Studien sind jedoch notwendig um diesen Zusammenhang zu beweisen.

\section{P1.002 ,Die kleine Milz، - Klinik, B-Bild und kontrastmittelunterstützte Sonografie (CEUS) bei Hyposplenie: Eine retrospektive Analyse bei $n=50$ Patienten}

\author{
Autoren Trenker $C^{1}$, Neesse $A^{2}$, Görg $C^{1}$ \\ Institut 1 Universitätsklinikum Marburg, Marburg/DE; 2 Innere Medizin, \\ Göttingen/DE \\ DOI 10.1055/s-0037-1606970
}

Problemstellung Im Gegensatz zur Splenomegalie ist der Krankheitswert der kleinen Milz (Hyposplenie) ungeklärt. B-Bild sonografische und CEUS Daten von größeren Kollektiven fehlen.

Patienten und Methode Im Zeitraum von Februar 2005- Januar 2017 wurden $\mathrm{n}=50$ Patienten mit Hyposplenie (Milzgröße $<7 \times 3 \mathrm{~cm}$ ) mittels B-Bild-Sonografie und CEUS untersucht. Es erfolgte eine retrospektive Analyse der B-Bild und CEUS- Daten hinsichtlich Ätiologie, Echogenität und Kontrastmittelverhalten der kleinen Milzen.

Ergebnis Bei $n=30(60 \%)$ der Patienten lag eine hämatologische Grunderkrankung (Knochenmarktransplantation $n=16$, Sichelzellanämie $n=6$, Lymphom $n=4$, sonstige $n=4$ ) vor. Unter den nicht-hämatologischen Grunderkrankungen $(n=20,20 \%)$ fanden sich $n=7$ solide Tumore, $n=6$ Autoimmunerkrankungen und $n=7$ Zufallsbefunde. B-Bild-sonografisch war die Echogenität der Milz normal (homogen echoarm) in $n=21$ (42\%) Patienten und pathologisch in $n=29$ Fällen (58\%), wobei zwischen inhomogen $n=29$ (58\%) und echoreich $n=9$ (18\%) Milztextur unterschieden wurde. In der CEUS zeigten $n=27$ Patienten (54\%) ein regelrechtes isoechogenes arterielles und parenchymales Enhancement und $n=23$ Patienten (46\%) ein pathologisches Enhancement in der arteriellen und/oder parenchymalen Phase im Sinne einer komplexen (arteriell und parenchymal $n=2$ ), fehlenden (arteriell $n=3$, parenchymal $n=6$ ) oder einer verminderten (arteriell $n=8$, parenchymal $n=15$ )
Anreicherung. In der Gruppe mit hämatologischer Grunderkrankung zeigten 57\% ein atypisches CEUS-Muster, unter den nicht-hämatologischen 30\%. Schlussfolgerung Die Hyposplenie ist häufig mit einer hämatologischen Grunderkrankung assoziiert. Das Symptom der Hyposplenie ist mit einer auffälligen B-Bild-Echotextur und CEUS-Muster assoziiert. Die klinische Bedeutung dieser Milzpathologie bleibt unklar.

\section{P1.003 MWTA - ein 8-Jahresrückblick}

Autoren Zimmermann $\mathrm{P}^{1}$, Will $\mathrm{U}^{1}$

Institut $1 \mathrm{SRH}$ Waldklinikum Gera, Gera/DE

DOI 10.1055/s-0037-1606971

Problemstellung Ergebnisbewertung der Mikrowellenthermoablation (MWTA) bei malignen Raumforderungen.

Patienten und Methode Ein 8-Jahresrückblick der durchgeführten MWTA's im SRH Waldklinikum Gera. Es erfolgte eine Aufarbeitung bezüglich der Erfolgsund Komplikationsrate. Das Resultat nach MWTA (Rezidiv oder avitale Nekrose) wurde mit Kontrastmittelultraschall (CEUS) in festgelegten Abständen kontrolliert.

Ergebnis Insgesamt wurden 84 MWTA's von Leberherden bei 57 verschiedenen Patienten durchgeführt (bei $n=15$ Ablationen, bei $n=5$ jeweils 3 Ablationen, bei $n=13$ jeweils 2 Ablationen, aufgrund von Rezidiven). Bei neun Patienten wurden mehrere Tumore in einer Sitzung abladiert (bei $n=2$ Patienten jeweils 3 Tumore, bei $n=7$ Patienten jeweils 2 Tumore) In der histologischen Aufarbeitung der zuvor erfolgten Leberpunktionen zeigten sich 8 verschiedene Entitäten ( 24 x HCC, 19 x KRK, 4 x Mamma-Ca, 6 x CCC, je einmal Nierenzell-Ca, Ovarial-Ca, Dünndarm-Ca und NET). Bei 4 Patienten wurden insgesamt 5 MWTA's ( 1 x Rezidiv) an der Niere bei Nierenzellkarzinom durchgeführt. Die Erfolgsrate im Allgemeinen lag bei $62 \%$ über alle Entitäten, wenn man die Pat. mit mehrfachen Ablationen aufgrund von Rezidiven herausrechnet bei $82 \%$. Es gab keine Todesfälle. Die Komplikationsrate schwerer Komplikationen lag bei 4,5\% (eine Hautnekrose, zwei Abszesse, eine Aspiration) und der leichten Komplikationen bei 7\% (4 Blutungen aus dem Stichkanal, ein Leberkapselhämatom, eine Einblutung in die Bauchdecke).

Schlussfolgerung Die MWTA ist eine gute Alternative bei Raumforderungen bis $5 \mathrm{~cm}$ Größe verschiedener Entitäten bei Patienten mit erhöhten operativen Risiko. Sie hat eine hohe Erfolgsrate bei niedriger Komplikationsrate. Auch die Nachsorge mit CEUS ist eine strahlungsfreie und nierenschonende Untersuchung mit niedriger Komplikationsrate.

\section{P1.004 Der Stellenwert bettseitig sonografisch erfassbarer Marker in der Dehydratations-Diagnostik bei älteren Patienten}

Autoren Diederich $\mathrm{H}^{1}$, Burkhardt $\mathrm{H}^{1}$

Institut 1 Universitätsmedizin Mannheim, Mannheim/DE

DOI 10.1055/s-0037-1606972

Problemstellung Die Diagnose Dehydratation ist bei älteren Patienten oft eine Herausforderung, da weder laborchemische Parameter noch klinische Zeichen eine große Spezifität aufweisen.

Patienten und Methode In einem zufällig ausgewählten Kollektiv älterer Patienten, die eine Notfallaufnahme aufsuchten wurden in einem bedside-Ansatz sonografisch erhebbare Maße erhoben und mit der klinischen Einschätzung verglichen. Da kein Gold-Standard der Diagnostik im Aufnahme-Setting abbildbar ist, wurde eine eine klinische Synopse verwendet, um dehydrierte Patienten zu identifizieren. Als Vergleich dienten nach dieser Einschätzung euhydrierte Patienten.

Ergebnis 78 Patienten mit einer nach klinischen Synopse eindeutige Dehydratation wurden mit 121 Kontrollen ohne Zeichen der Dehydratation verglichen. Es zeigten sich signifikant Unterschiede zwischen diesen beiden Gruppen was Kompressibilität der Vena cava, ihre maximale und minimale Exkursion im M-Mode sowie den Diameter im Inspirationsmanöver anbelangt $(<0,001)$. Allerdings ergaben sich in einer ROC-Analyse nur mäßige Werte für die dia- 
gnostische Effizienz. Der beste Wert fand sich für den inspiratorischen Diameter mit $A \cup C=0,73$.

Schlussfolgerung Sonografische Diagnostik kann die Diagnose einer Dehydratation unterstützen und ist im bed-side-Ansatz gut durchführbar. Die Diagnostik der Dehydratation bleibt insgesamt aber schwierig und wird am besten aus einer Synopse unterschiedlicher, sowohl apparativer wie klinischer Marker heraus gestellt.

\section{P1.005 Sonografische Diagnose eines Phytobezoar-lleus mit post-operativer, sekundärer Pneumatosis intestinalis}

Autoren Greger $\mathrm{A}^{1}$, Konermann $\mathrm{M}^{2}$

Institut 1 Agaplesion Diakonie Kliniken Kassel, Kassel/DE;

2 Marienkrankenhaus Kassel, Kassel/DE

DOI 10.1055/s-0037-1606973

Problemstellung Phytobezoare sind ein nicht-lösliches Konglomerat aus Pflanzenfasern im Gastrointestinal-Trakt. Sie sind eine seltene Ursache meist unspezifischer abdomineller Beschwerden, und werden in ca. 0,068\% -0,43\% der Gastroskopien gefunden. Die Therapie besteht üblicherweise aus einer Kombination aus einer Lyse mit Coca Cola ${ }^{\circledR}$ und/oder Enzymen sowie endoskopischer Fragmentation. Als Komplikation des Bezoars kann es u. a. zu einer intestinalen Obstruktion kommen. Insbesondere die Migration nach einer vorherigen Lyse-Therapie ist hierbei eine nur selten beschriebene Komplikation. Patienten und Methode Der Patient stellte sich nach der Diagnose eines gastralen Phytobezoars unter laufender Coca Cola ${ }^{\circledR}$-Lyse-Therapie im Verlauf mit einem Dünndarm-lleus vor. Sonografisch konnte der Bezoar im terminalen Ileum als Ursache bestätigt werden.

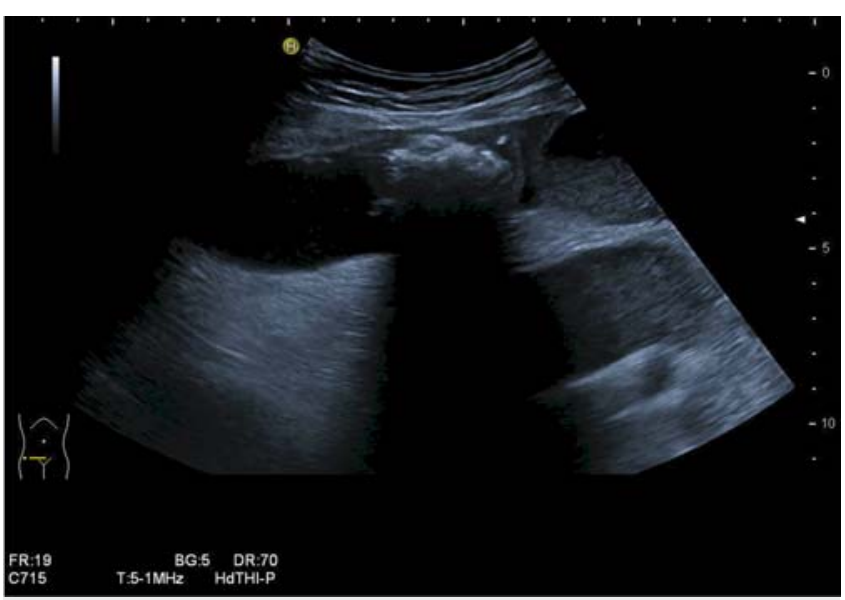

Abb. 1 Intestinale Obstruktion des terminalen lleum durch Phytobezoar

Ergebnis Nach primär erfolgreicher Resektion mittels lleotomie stellte sich nach wenigen Tagen sonograpisch ein post-operativer lleus mit sekundärer Pneumatosis intestinalis dar. Unter konservativer Therapie kam es zur restitutio ad integrum.

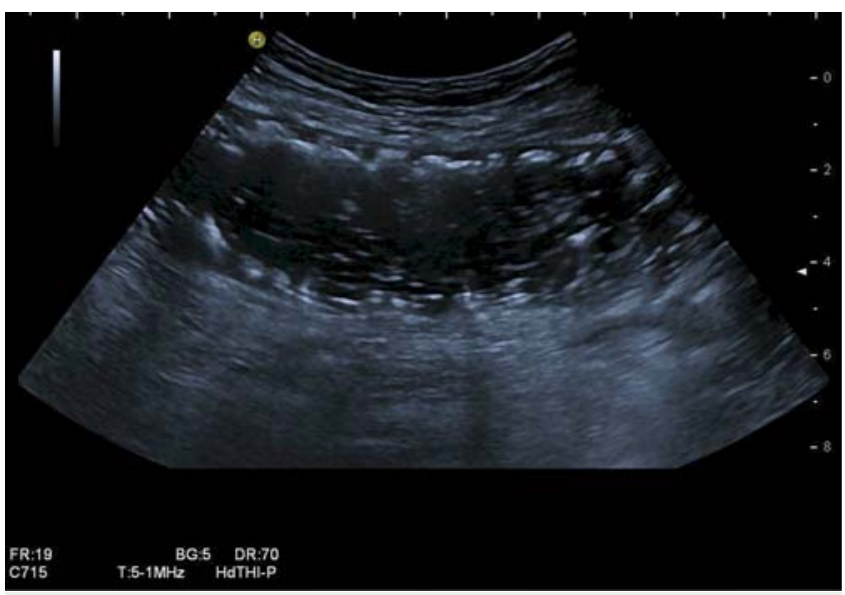

Abb. 2 Pneumatosis intestinalis des Dünndarms

Schlussfolgerung Nur ca. 0,4\% - 9\% der lleus-Fälle sind durch Bezoare bedingt. Eine Migration nach suffizienter lytischer Therapie mit konsekutiver intestinaler Obstruktion wurde nur sehr selten beschrieben, sollte aber bei Kenntnis der Diagnose als relevante Komplikation der konservativen Therapie des Phytobezoars bedacht werden.

\section{P1.006 B-Bild Sonografie (US), kontrastmittelunterstützte Sonografie (CEUS) und Histologie von omentalen Raumforderungen: eine retrospektive Analyse von $\mathbf{n}=\mathbf{2 8}$ Patienten}

Autoren Trenker $C^{1}$, Neesse $A^{2}$, Görg $C^{1}$

Institut 1 Universitätsklinikum Marburg, Marburg/DE; 2 Innere Medizin, Göttingen/DE

DOI 10.1055/s-0037-1606974

Problemstellung Eine omentale Verdickung gilt als charakteristisches Zeichen einer Peritonealkarzinose. US und CEUS Muster von tumorsuspekten omentalen Raumforderungen (RF) werden dargestellt.

Patienten und Methode Im Zeitraum von 2008 - 2016 wurden $n=28$ Patienten mit US detektierten omentaler RF > $1 \mathrm{~cm}$ Tumordicke mittels CEUS untersucht und anschließend biopsiert. Bei $n=15$ (53,6\%) bestand eine Tumoranamnese, bei $n=13(46,4 \%)$ keine. Retrospektiv wurden die RF hinsichtlich Tumordicke, Echogenität und Kontrastmittelverhalten analysiert.

Ergebnis $\mathrm{N}=24$ (85,7\%) der omentalen RF waren maligne ( $\mathrm{n}=22$ Metastasen, $\mathrm{n}=2 \mathrm{NHL}$ ) und $\mathrm{n}=4(14,3 \%)$ benigne ( $\mathrm{n}=2$ Fettgewebsnekrosen, $\mathrm{n}=2$ Inflammationen). $\mathrm{N}=17$ (60,7\%) Patienten hatten Aszites. Die Tumordicke war $<2 \mathrm{~cm}(\mathrm{n}=17,60,7 \%)$ bzw. $>2 \mathrm{~cm}(\mathrm{n}=11,39,3 \%)$. Die malignen RF stellten sich im US echoarm $(n=9 ; 37,5 \%)$ und echoreich $(n=15 ; 62,5 \%)$ im Vergleich zum umgebenden Fettgewebe dar mit Strukturinhomogenität ( $n=18 ; 75 \%$ ), sowie zusätzlich fokalen Herden in der RF ( $n=4 ; 16,7 \%)$. In der CEUS zeigten die malignen Läsionen arteriell ein Hyper- $(n=4 ; 16,7 \%)$, Iso- $(n=16 ; 66,7 \%)$ oder Hypo-enhancement $(n=4 ; 16,7 \%)$, sowie parenchymal ein Hyper- $(n=2$; $8,3 \%$ ), Iso- ( $n=1 ; 4,2 \%$ ) oder Hypoenhancement ( $n=21 ; 87,5 \%)$.

Im US waren die benignen Tumorformationen echoarm ( $\mathrm{n}=2 ; 50 \%)$ und echoreich ( $n=2 ; 50 \%$ ) und hatten zusätzlich Aszites ( $n=1 ; 25 \%$ ). In der CEUS zeigten die RF arteriell und parenchymal ein Hyper- $(n=2 ; 50 \%)$ Hypo( $n=1 ; 25 \%$ ) oder fehlendes Enhancement ( $n=1 ; 25 \%$ ).

Schlussfolgerung Tumorsuspekte omentale RF waren überwiegend maligner Genese $(85,7 \%)$. Sie sind in der CEUS durch ein parenchymales Auswaschen $(87,5 \%)$ charakterisiert. Lediglich die Tumordicke erscheint zur Dignitätseinschätzung omentaler Tumore hilfreich zu sein. Zur Diagnosesicherung sollte eine histologische Sicherung erfolgen. 


\section{P1.007 Ultraschallgezielte Fiducialanlage zur Steuerung hochfokussierter Strahlentherapie}

Autoren Schröder $\mathrm{W}^{1}$, Becker $\mathrm{M}^{2}$, Bleif $\mathrm{M}^{3}$, Schuler $\mathrm{A}^{1}$

Institut 1 Alb Fils Kliniken, Geislingen/DE; 2 Alb Fis Kliniken, Göppingen/DE;

3 Alb Fils Kliniken Göppingen, Göppingen/DE

DOI 10.1055/s-0037-1606975

Problemstellung Das Cyberknife ist eine gute Möglichkeit der hochfokusierten Strahlentherapie.

Die Bewegungen der Zielstruktur bzw. des Tumors aufgrund von Atmung oder Organverschiebungen (Darm, Leber, Prostata, Niere) müssen bei der Behandlung berücksichtigt werden. Deshalb werden die Lage des Organs und damit auch die Position des Tumors während der Behandlung ständig kontrolliert.

Da die inneren Organe keinen ausreichenden Kontrast in den Positions-Röntgenaufnahmen liefern, werden kleine Metallstifte aus Gold von wenigen Millimetern Größe in oder um den Tumor platziert. Dies geschieht vor der Bestrahlungsplanung in örtlicher Betäubung. Die Goldmarker (sogenannte „Fiducials“) werden als Referenzmarkierung in ihrer Lagebeziehung zum Tumor vom Röntgensystem während der Behandlung abgebildet.

Die ultraschallgezielte Platzierung solcher Marker wird unter transkutaner Lokalanästhesie oder in Propofol-Kurznarkose endosonografisch durchgeführt und ist das hierzu am schnellsten durchführbare Verfahren.

Patienten und Methode Im Zeitraum August 2014 bis April 2017 wurden in der Helfenstein Klinik Geislingen bei 26 Patienten Fiducialanlagen durchgeführt. Diese wurden prospektiv bezüglich technischem Erfolg und Komplikationsrate erfasst.

Die 26 Patienten wurden mit insgesamt 33 Goldfiducials verschiedener Hersteller versorgt. Sieben Anlagen erfolgten endosonografisch, die restlichen wurden durch transkutanen Ultraschall gestützt durchgeführt.

Es erfolgten Anlagen in Leber, Niere, Lymphknoten, Retroperitoneum, Pankreas, und Beckenweichteilen.

Ergebnis Die technische Erfolgsrate lag bei $100 \%$. Hierbei kam es bei zwei Patienten zu lokalen Schmerzereignissen aber ansonsten zu keinen weiteren erfassten Komplikationen. Die möglichen Blutungs-Komplikationen waren, wie aus der DEGUM-Studie zu erwarten, niedrig. (1)

Schlussfolgerung Zusammenfassend kann festgehalten werden, dass die ultraschallgezielte Anlage von Fiducials zur Steuerung einer hochfokussierten Strahlentherapie (Cyberknife) mittels dynamischer Positionskontrolle sicher, schnell und komplikationsarm sowohl transkutan als auch endosonografisch durchgeführt werden kann.

\section{P1.008 Evaluation der Anwendbarkeit der Fusions-Kontrastmittelsonografie zur Charakterisierung von in der CT unklar gebliebenen Raumforderungen}

\author{
Autoren Ignee $A^{1}$, Käb $A^{1}$, Schuessler $G^{1}$, Dietrich $\mathrm{CF}^{2}$ \\ Institut 1 Caritas-Krankenhaus, Bad Mergentheim/DE; 2 christoph. \\ dietrich@ckbm.de, Bad Mergentheim/DE \\ DOI 10.1055/s-0037-1606976
}

Problemstellung In häufiger werdender Frequenz wird die Kontrastmittelsonografie zur Charakterisierung von in der CT unklar gebliebenen Raumforderungen angefragt. Da sowohl nur in der CT als auch nur in der Sonografie sichtbare Raumforderungen vorkommen ergibt sich hier die Notwendigkeit sicherzustellen dass die in der Sonografie gesehene RF der mittels CT unklar gebliebenen entspricht. Ein Augenmerk muss darauf gelegt werden, dass hier die Fusionspunkte resp. die Fusionsebene die angefragte Raumforderung nicht mit einbeziehen.

Patienten und Methode Über einen Zeitraum von 3 Jahren wurden konsektutive Patienten in die Studie eingeschlossen, welche aufgrund von in der CT gesehenen unklaren Raumforderungen zur Charaktierisierung mittels Kontrastmittelsonografie zugewiesen wurden. Die Untersuchung erfolgte un- geblindet an der GE Logiq E9 nativ und nach Kontrasmittelinjektion (2,4 ml Sonovue)

Ergebnis In der Mehrzahl der Fälle war die Fusionssonografie erfolgreich. In einer signifikanten Anzahl allerdings gelang die technische Fusion nicht. Die relevanten Einflussfaktoren sind: Größe der Läsion, Position der Läsion, Darstellbarkeit der Läsion im B-Bild.

Schlussfolgerung Die Fusionssonografie existiert seit mehreren Jahren auf dem Markt. Die Machbarkeit der Fusionssonografie steht außer Frage. Um aus der Nischenfuktion einer ästhetischen Methode herauszukommen ist die Etablierung von eindeutigen Indikationen erforderlich. Diese sind bislang nicht in ausreichendem Maße evaluiert worden. In der vorliegenden Arbeit versuchen wir zu zeigen, in welcher Häufigkeit und unter welchen Voraussetzungen eine Fusion ohne Inanspruchnahme der Läsion selbst als Fusionspunkt eine eindeutige Zuordnung von der zu charakterisierenden Läsion gelingt.

\section{P1.009 22-jähriger allogentransplantierter Patient bei ALL mit klinischer Diagnose einer veno-occlusive disease (VOD) und nahezu fehlendem Kontrastmittelenhancement der Leber in der CEUS: Hinweis auf pathologische Vaskularisation der Leber}

Autoren Trenker $C^{1}$, Sohlbach $K^{2}$, Görg $C^{1}$

Institut 1 Universitätsklinikum Marburg, Marburg/DE; 2 UKGM Marburg, Marburg/DE

DOI 10.1055/s-0037-1606977

Patienten und Methode Tag 16 nach allogener Stammzelltransplantation zeigte ein 22-jährigem Patienten mit ALL einen Anstieg der Transaminasen (ALT max. $97 \mathrm{U} / \mathrm{I}$ ), Cholestaseparameter (Bilirubin gesamt max. 4,3 mg/d), eine Gerinnungseinschränkung (Quick 74\%), sowie eine deutliche Gewichtszunahme von 10 Kilogramm. B-Bild sonografisch imponierte eine Hepatomegalie, perihepatischer Aszites, eine Splenomegalie $(16 \times 6 \mathrm{~cm})$, ein Gallenblasenwandödem und Pleuraergüsse. Die Pfortader zeigte ein pulsatiles Flussprofil mit verminderter Flussgeschwindigkeit (Vmax $16 \mathrm{~cm} / \mathrm{s}, V \min 4 \mathrm{~cm} / \mathrm{s}$, Vmean $10 \mathrm{~cm} / \mathrm{s}$ ). Bei Verdacht auf VOD erfolgte die Therapieeinleitung mit Defibrotide. In der CEUS zeigte die Leber eine vorhandene arterielle und portalvenöse Kontrastmittelanflutung aber mit nahezu fehlender Kontrastmittelanreicherung in der portalvenösen und parenchymalen Phase im Vergleich zur Milz. Unter Therapie besserten sich Klinik, Laborwerte. Die sonografischen Befunde normalisierten sich im Verlauf von 14 Tagen, mit nun normwertig monophasischem Flussprofil $(21 \mathrm{~cm} / \mathrm{s})$ der Pfortader und deutlich gebesserter Kontrastmittelanreicherung des Leberparenchyms.

Schlussfolgerung Die VOD ist ein lebensbedrohliches und allein klinisch schwer diagnostizierbares Krankheitsbild. Eine Abgrenzung zu Differenzialdiagnosen wie Leber GvHD und toxischer Leberfunktionsstörung ist notwendig. Die Diagnosestellung und Therapieeinleitung erfolgt gewöhnlich klinisch ohne histologische Diagnosesicherung. Die B-Bild sonografischen Zeichen der VOD mit Nachweis eines vermindertem Pfortaderfluss, Hepatomegalie und Aszitesbildung gelten als hilfreich. CEUS-Untersuchungen bei VOD sind nicht bekannt. Das hier erstmals beschriebene pathologische CEUS Muster mit nahezu fehlendem Leberenhancement lässt auf eine Mikrovaskularisationsstörung der Leber schließen und ist möglicherweise ein zusätzlicher bildgebender Indikator zur Diagnose der VOD. 


\section{P 2: Poster Interdisziplinär \\ Poster Präsentation}

\section{P2.001 Beeinflussung der diastolischen \\ Ventrikelfuktion durch einen einwöchigen Urlaub mit unterschiedlichen Aktivitätsprogrammen (Nordic Walking, E-Bike, Golfen)}

Autoren Lechleitner $\mathrm{P}^{1}$

Institut 1 BKH Lienz, Lienz/AT

DOI 10.1055/s-0037-1606978

Problemstellung Zu den Auswirkungen eines einwöchigen Aktivitätsurlaubes auf Herz-Kreislaufparameter ist wenig bekannt. Wir haben eine prospektive, offene Vergleichsstudie mit folgender Fragestellung durchgeführt: Was bewirkt ein 1-wöchiger Aktivurlaub mit den Trendsportarten Nordic-Walking, elektounterstütztes Biking (E-Biking) und Golf auf die diastolische Ventrikelfunktion.

Patienten und Methode Es wurden 30 Golfer und je 11 Nordic-Walker bzw. 11 E-Biker (NWE-Gruppe) eingeschlossen.

Echokardiografisch (Vivid 7; GE) wurden erhoben: E/A-Verhältnis, E:e', linksventrikulärer Tei-Index, Dezellerationszeit, linksventrikuläre Auswurfsfraktion (Simpson rule).

Ergebnis Die Ergebnisse werden als Median angegeben.

Golfgruppe: Bei der diastolischen Funktion des linken Ventrikels (s. Tab. 1) verbesserte sich E: e' von 7,9 auf 7,7 sowie der linksventrikuläre Tei-Index von 0,61 auf 0,51 (n. s.).

Nordic-Walking und E-Bike-Gruppe: Es kam zu einer signifikanten Verbesserung der diastolischen Herzfunktion, wobei sich E: e' von 7,6 auf 7,2 ( $p=0,02)$ und der linksventrikuläre Tei-Index von 0,64 auf 0,45 ( $p=0,003)$ verminderte.

Tab. 1 Echokardiografische Parameter vor und nach der Aktivitätswoche.

\begin{tabular}{|c|c|c|c|c|c|}
\hline Golf & vor & nach & NWE & vor & nach \\
\hline EF (\%) & 61 & 61 & EF (\%) & 61 & 61 \\
\hline $\mathrm{E}: \mathrm{A}$ & 1,0 & 1,1 & $\mathrm{E}: \mathrm{A}$ & 1,1 & 1,2 \\
\hline$E: e^{\prime}$ & 7,9 & 7,7 & $\mathrm{E}: \mathrm{e}^{\prime}$ & 7,6 & 7,2 \\
\hline LV-Tei & 0,61 & 0,52 & LV-Tei & 0,64 & 0,45 \\
\hline Dec (ms) & 237 & 244 & Dec (ms) & 240 & 252 \\
\hline
\end{tabular}

Schlussfolgerung Die erstmals bei körperlicher Aktivität von Urlaubern untersuchte diastolische Links-Ventrikelfunktion, zeigt in der Golfgruppe eine tendenzielle, aber nicht signifikante und in der NWE-Gruppe eine signifikante Verbesserung der Parameter. Dies ging einher mit einer geringen Reduktion des NTproBNP Wertes in der Golfgruppe. Es scheint überraschend, dass bereits nach einer einwöchigen körperlichen Aktivität Auswirkungen auf die diastolische Funktion fassbar wurden.

\section{P2.002 Darstellung pleuraständiger Lungenherde mittels Bildfusion von CT und Ultraschall}

Autoren Klinger $C^{1}$, Grotz $A^{1}$, Kruse $A^{1}$, Fröhlich $E^{2}$, Riecken $B^{1}$ Institut 1 Klinikum Ludwigsburg, Ludwigsburg/DE; 2 Universität Tübingen, Tübingen/DE

DOI 10.1055/s-0037-1606979

Problemstellung Sonografisch gesteuerte Interventionen am Thorax beschränken sich bisher auf pleuraständige Läsionen. Neue Möglichkeiten eröffnet evtl. die Fusion von CT- oder MRT-Bildern mit einer sonografischen Echtzeituntersuchung, wodurch auch nicht pleuraständige Läsionen einer sonografisch gesteuerten Intervention zugänglich gemacht werden könnten. Ziel dieser Arbeit ist die erstmalige Überprüfung der Möglichkeit einer fusionssonografischen Darstellung pleuraständiger Lungenherde.

Patienten und Methode Von Februar 2017 bis April 2017 wurden 6 Patienten mit 7 pleuraständigen Lungenherden (entzündlich, $n=1$; diffus großzelliges B-NHL, $\mathrm{n}=2$; NSCLC, $\mathrm{n}=2$; Melanom-Metastase, $\mathrm{n}=1$; Pankreaskarzinom-Me- tastase, $\mathrm{n}=1$ ) mittels Fusionssonografie (Siemens Acuson S3000, 6C1HDSchallsonde) in Rückenlage untersucht.

Bei allen Patienten wurden 3 der folgenden Referenzpunkte für die Fusion verwendet: Processus xiphoideus $(n=4)$, Aufzweigung rechter Pfortaderast $(n=4)$, Abgang Truncus coeliacus $(n=3)$, rechter Nierenoberpol $(n=3)$, Lebervenenstern ( $n=2)$, Sternoclaviculargelenk rechts $(n=1)$, Portreservoir $(n=1)$. Ergebnis Der Zeitraum zwischen Computertomografie und Fusionssonografie betrug zwischen 1 und 11 Tage (Median 3,5 Tage). Eine Patientin hatte einen ausgeprägten Pleuraerguss, welcher jedoch zwischen CT und Fusionssonografie nicht punktiert wurde. 6/7 Läsionen (85,7\%) ließen sich gut mittels Fusionssonografie darstellen (Abb. 1 und 2). Die Größe dieser Läsionen lag zwischen 8 und 49 mm (Median $34 \mathrm{~mm}$ ). Schlecht fusionierbar war ein Rundherd (14,3\%), welcher nur durch maximale Kippung von kaudal transhepatisch angelotet werden konnte (Abb. 3).

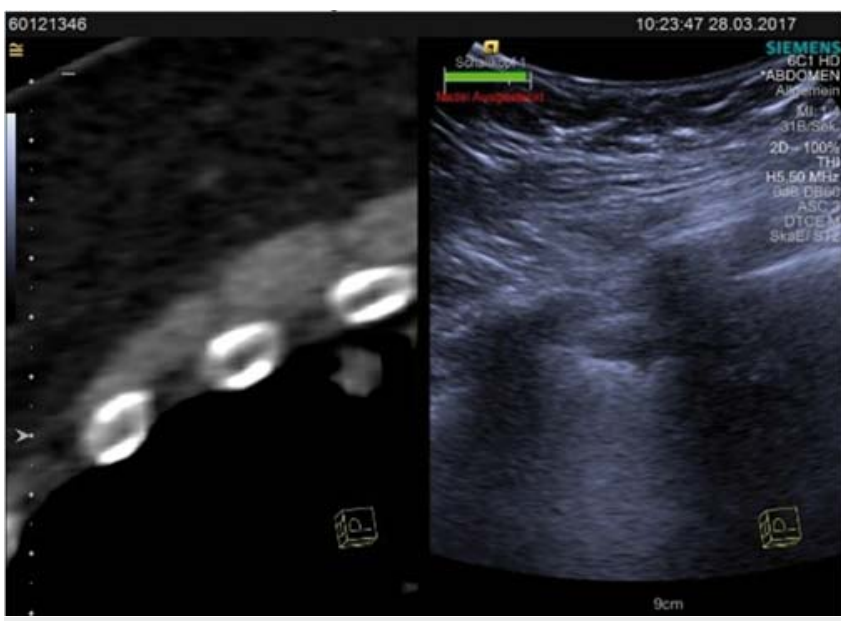

Abb. 1 Erfolgreiche Fusion eines $8 \mathrm{~mm}$ großen, pleuraständigen Lungenherdes im Bereich der vorderen Axillarlinie links.

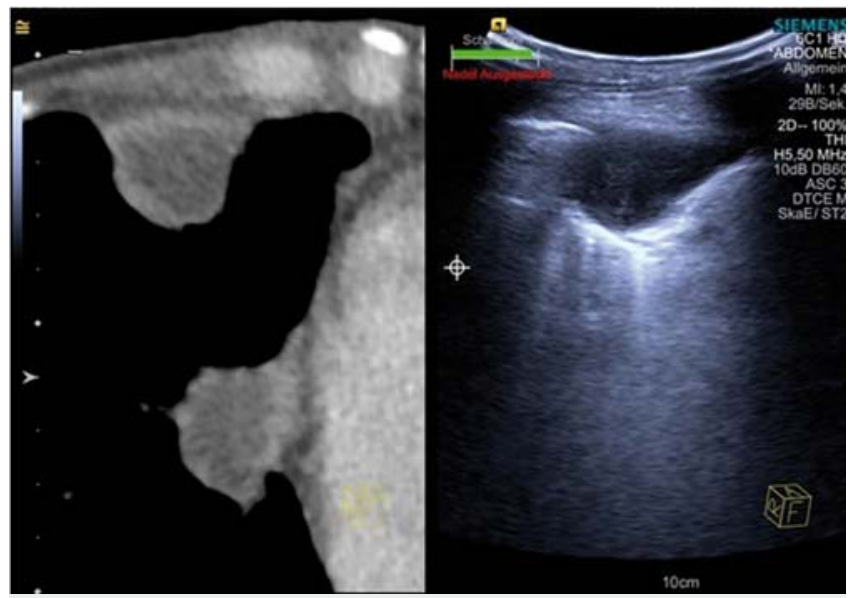

Abb. 2 Erfolgreiche Fusion eines pleuraständigen Lungenherdes im Bereich der Medioklavikularlinie rechts. 


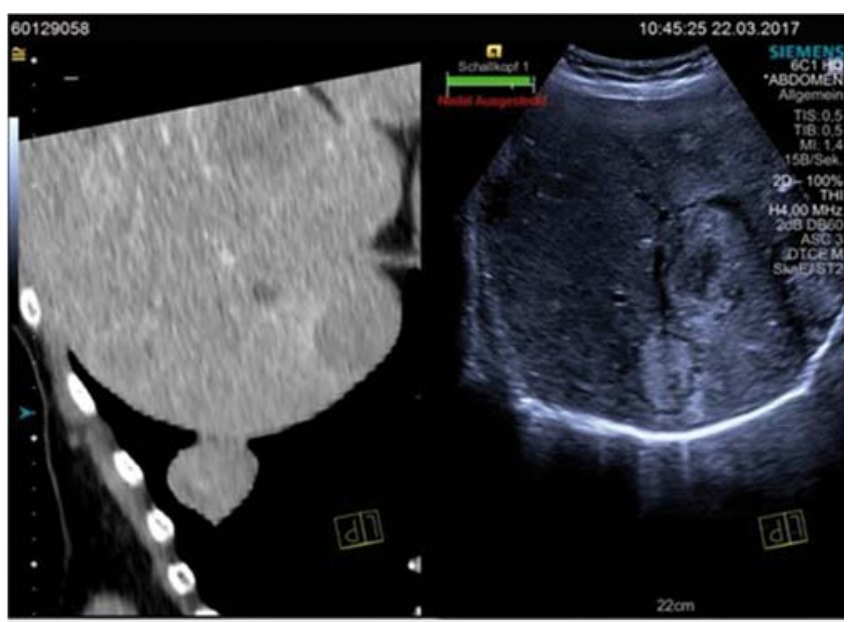

Abb. 3 Fehlende Fusion eines zwechfellnahen, nur durch maximale Schallkopfkippung von kaudal transhepatisch anlotbaren Lungenherdes.

Schlussfolgerung Die fusionssonografische Darstellung auch kleiner pleuraständiger Lungenherde ist bei orthograder Anlotung gut machbar. Schwierigkeiten bereiten hingegen Herde, welche nur durch maximale Schallkopfbewegungen erreichbar sind. Weitere Untersuchungen sind erforderlich, um zu überprüfen, ob in Zukunft mittels fusionssonografischer Steuerung auch subpleural gelegene Lungenherde sicher perkutan biopsiert werden können.

\section{P2.003 Schilddrüsensonografie bei amiodaroninduzierter Hyperthyreose}

Autoren Fernandez Sanchez J ${ }^{1}$

Institut 1 Robert-Bosch-Krankenhaus Stuttgart, Stuttgart/DE

DOI 10.1055/s-0037-1606980

Problemstellung Eine Nebenwirkung von Amiodaron ist die Entwicklung einer Hyperthyreose. Ziel dieser Studie ist eine Evaluierung des sonografischen Musters der Schilddrüse (SD) bei amiodaroninduzierter Hyperthyreose (AIH).

Patienten und Methode Bei 29 Patienten mit AlH wurde eine hochauflösende Sonografie und Color Doppler der SD durchgeführt. Vor der Amiodaronbehandlung war das TSH nicht supprimiert. Das Echomuster, Vaskularisation und die Präsenz von SD-Läsionen wurde evaluiert. Die Einteilung der AlH (Typ I, II, Mischform) wurde mittels SD-Szintigrafie mit 99 m.Tc-MIBI festgestellt.

Ergebnis 16 Patienten mit AlH Typ I, 5 mit Typ II und 8 mit Mischform.12/16 AlH Typ I zeigten Knoten (nicht suspekt, TIRADS 2 oder 3) mit einem weitgehend normalen Muster und normaler Vaskularisation des paranodulären SDGewebes. Bei 4/16 AlH Typ I lagen keine SD-Knoten vor und das Echomuster, sowie die Perfusion waren normal. 5 AlH Typ2 und 8 AlH Mischform zeigten ein inhomogenes Muster, leicht echoreich bis echoarm, ohne relevante Vaskularisation und ohne Knoten.

Schlussfolgerung In Patienten mit AlH kann beim sonografischen Nachweis von Knoten mit normalem Echomuster und Vaskularisation der SD eine AlH Typ I aufgrund einer multifokalen bzw. fokal-disseminierten SD-Autonomie diagnostiziert werden. Bei fehlendem Nachweis von fokalen SD-Läsionen mit normalem Echomuster und normaler Vaskularisation kann sonografisch den V.a. AlH Typ I bei einer disseminierten SD-Autonomie geäußert werden. Eine szintigrafische Bestätigung ist jedoch erforderlich. Eine leicht echoreiche bis echoarme SD ohne Knoten und ohne Vaskularisation ist vereinbar mit AlH Typ II, jedoch kann in diesen Fällen eine Mischform der AlH sonografisch nicht sicher ausgeschlossen werden, sodass hier eine weitere szintigrafische Abklärung notwendig ist.

\section{P2.004 25 Jahre Alkoholinstillation in hyperfunktionelle Schilddrüsenknoten}

Autoren Blank $W^{1}$, Müller $T^{1}$, Schuler $A^{2}$, Kubicka $S^{1}$, Braun $B^{1}$ Institut 1 Klinikum am Steinenberg, Reutlingen/DE; 2 Helfensteinklinik, Geislingen/DE DOI 10.1055/s-0037-1606981

Problemstellung Die sonografisch gesteuerte perkutane Ethanol-InstillationsTherapie (PEIT) von Schilddrüsenerkrankungen wurde 1990 von italienischen Arbeitsgruppen eingeführt. PEIT wird international in Guidelines in der Sklerosierung von rezidivierenden Schilddrüsenzysten als Therapie der ersten Wahl empfohlen, und seit 1990 weltweit als Alternative zu Operation bzw. RadiojodTherapie zur Zerstörung hyperfunktioneller Schilddrüsenknoten eingesetzt. Da mehrere Sitzungen notwendig sind und die Rezidivraten in einigen Follow-upStudien hoch waren, wurde PEIT in einigen Zentren durch Radiofrequenzablation (RFA) ersetzt.

Patienten und Methode In der Reutlinger Klinik wird die PEIT seit 25 Jahren bei einem selektionierten Krankengut (Operation, Radiojodtherapie nicht möglich, Patientenwunsch) durchgeführt. In unserer prospektiv angelegten Studie wurden die Erfolge nach Therapieende von 268 Patienten, der Jahren 1992 bis 2006 ausgewertet (G1) und mit 51 Pat. der Jahre 2010 bis 2015 verglichen (G2). In G2 wurde im Therapiemonitoring zusätzlich zur Farbdopplersonografie die Kontrastmittelsonografie (CEUS) eingesetzt. G1: 155 Pat. hatten vor Therapie eine manifeste Hyperthyreose (toxische Adenome, $t A$ ), 113 Pat. eine latente Hyperthyreose (prätoxische Adenome, pA). G2: 20 Pat. tA, 31 Pat. pA.

Ergebnis G1: Bei 133 Pat. (86\%) mit tA wurde eine Euthyreose erreicht, bei 81 Pat. (72\%) mit pA kam es zu einem TSH-Anstieg in Normalbereiche. G2: 19 Pat. (95\%) mit tA erreichten eine Euthyreose, 29 Pat (94\%) mit pA einen TSHAnstieg.

Schlussfolgerung Durch den zusätzlichen Einsatz der Kontrastmittelsonografie im Therapiemonitoring kann die Erfolgsrate der PEIT bei hyperfunktionellen Schilddrüsenknoten deutlich verbessert werden.

\section{P2.005 Doppler-Sonografie zur Beurteilung des venösen Rückflusses bei kraftvoller Aktivierung der Wadenpumpe im Sitzen}

Autoren Guthoff $A E^{1}$, Kropp $A T^{1}$, Guth $S^{1}$, Meiss $A L^{2}$, Bamberger $\mathrm{CM}^{1}$

Institut 1 Medizinisches Präventionszentrum Hamburg am

Universitätsklinikum Hamburg-Eppendorf, Hamburg/DE;

2 Universitätsklinikum Hamburg-Eppendorf, Hamburg/DE

DOI 10.1055/s-0037-1606982

Problemstellung Die mit Immobilität durch Reisen, Krankheit oder Arbeit einhergehende venöse Stase stellt einen wichtigen Risikofaktor für die Entstehung einer Becken-Beinvenenthrombose dar. Daher haben wir uns die Frage gestellt, in welchem Maße unterschiedliche Fußübungen zur Aktivierung der Wadenpumpe den venösen Rückfluss verbessern können.

Patienten und Methode 20 gesunde Probanden zwischen 22 und 65 Jahren führten in sitzender Position unterschiedliche Fußübungen durch. Der systolische Spitzenfluss in der Vena poplitea wurde bei jeder einzelnen Übung mittels Doppler-Sonografie bestimmt. Auf ein intraluminal positioniertes DopplerGate und einen Doppler-Winkel $\leq 60$ Grad wurde geachtet. Um die Beziehung zwischen Kraftaufwand und venösem Rückfluss zu korrelieren, verwendeten wir eine Kraftmessmatte (novel $\mathrm{GmbH}$ ) mit Zielvorgabe 100 und 250 Newton. Ergebnis Trotz der Bewegungsunruhe bei den kraftvoll durchgeführten Fußbewegungen gelang die Messung des systolischen Spitzenflusses in der Vena poplitea problemlos. Die gemittelten systolischen Spitzenflüsse (SS in cm/ Sekunde) ergaben: Ruhefluss SS = 5,6; Plantarflexion des Fußes mit 100 und 250 Newton Vorfußkraft SS=89,9 und 154,5; kraftvolle Plantarflexion des Fußes SS=91,0; kraftvolle Dorsalflexion des Fußes SS=193,6; kraftvolle Fersenhebung =107,4; kraftvolles Zehenkrallen SS =118,7. 
Schlussfolgerung Die Doppler-Sonografie ist ein geeignetes Messverfahren, um den venösen Rückfluss im Bein während gezielter Fußbewegungen beurteilen und vergleichen zu können. Alle durchgeführten Übungen führten zu einem signifikanten Anstieg des systolischen Spitzenflusses in der Vena poplitea. Der höchste Fluss wurde durch die kraftvolle Dorsalflexion, die Plantarflexion mit 250 Newton und ein kraftvolles Zehenkrallen des Fußes erreicht. Eine Empfehlung zur mechanischen Thromboseprophylaxe sollte diese Übungen einschließen.

\section{P2.006 Strömungsregulation der A. vertebralis}

Autoren Dähne $\mathrm{F}^{1}$, Schmehl $\mathrm{I}^{1}$

Institut 1 Unfallkrankenhaus Berlin, Berlin/DE

DOI 10.1055/s-0037-1606983

Problemstellung Der für den hinteren Kreislauf maßgebliche Volumenstrom (Q) der A. vertebralis (VA) bds. ergibt sich aus dem Produkt von Gefäßquerschnitt (A) und Strömungsgeschwindigkeit. Deren Regulationsverhalten ist Gegenstand dieser Arbeit.

Patienten und Methode Bei routinemäßigen Duplexsonografien von $371 \mathrm{~Pa}$ tienten im Alter von 62,5 $\pm 17,2 \mathrm{~J}$. mit regelrechter CT- oder MR-Angiografie und duplexsonografischem Normalbefund im hinteren Kreislauf wurde die enddiastolische Spitzengeschwindigkeit (v) und der Durchmesser (d) in V2 oder V1 im Doppler und Color Mode für beide VA erfasst.

In der enddiastolischen laminaren, quasistationären Strömungsphase ergeben sich nach Hagen-Poiseuille:

$\mathrm{Q}=5 \mathrm{v}^{*} \mathrm{~A}[\mu \mathrm{l} / \mathrm{s}]$, wobei $\mathrm{A}=\pi / 4^{*} \mathrm{~d}^{2}\left[\mathrm{~mm}^{2}\right]$

und das Druckverlustmaß dP' $=\mathrm{dP} / \mathrm{k}=5 \mathrm{v} / \mathrm{A}\left[\mathrm{mm}^{-1 *} \mathrm{~s}^{-1}\right]$, wobei in $\mathrm{k}$ Gefäßmessstrecke und Blutviskosität eingehen.

Ausgehend vom Mittelwert $A=7,65 \mathrm{~mm}^{2}$ (resp. $d=3,1 \mathrm{~mm}$ ) wurden die errechneten Gefäßquerschnitte in Gruppen unterteilt und in diesen die Mittelwerte für $\mathrm{v}, \mathrm{Q}$ und $\mathrm{dP}$ ' bestimmt.

Ergebnis

1. Q nimmt stetig, scheinbar linear mit $A$ zu.

2. $v$ nimmt stetig, scheinbar linear mit $A$ zu, jedoch nur bis wenig oberhalb des mittleren Gefäßquerschnitts. Darüber steigt v nicht weiter an.

3. dP' fällt mit A mit abnehmender Steilheit.

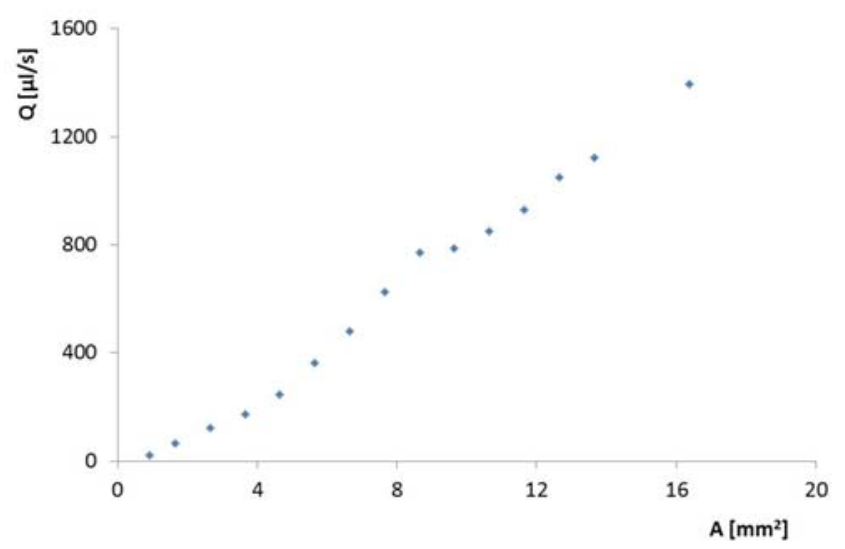

Abb. 1 Volumenstrom Q in Abhängigkeit vom Gefäßquerschnitt A

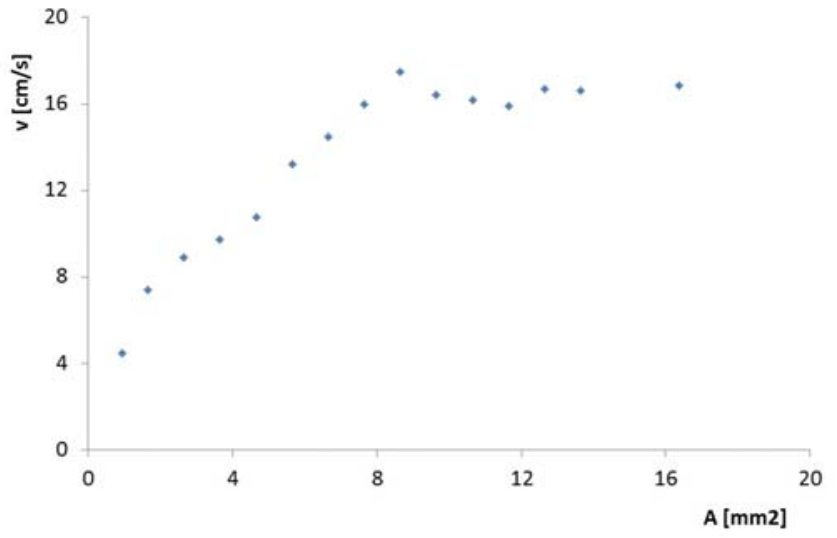

Abb. 2 Enddiastolische Spitzengeschwindigkeit v in Abhängigkeit vom Gefäßquerschnitt A

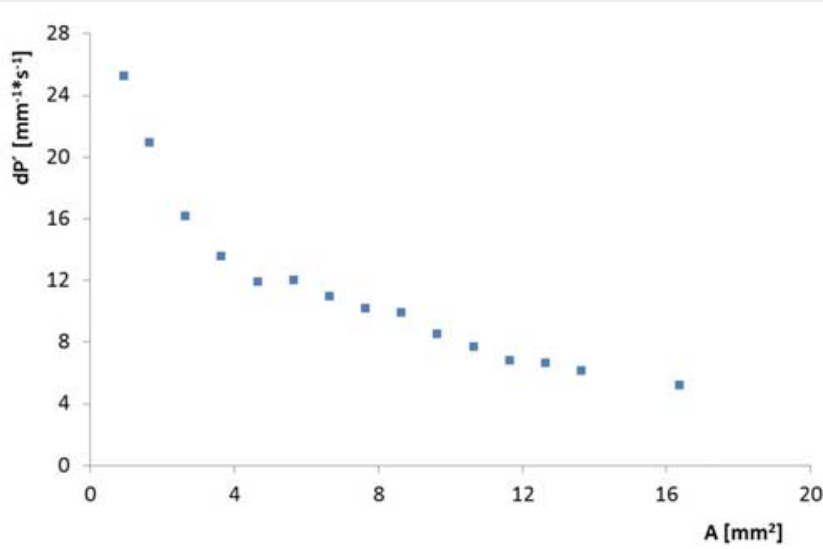

Abb. 3 Druckverlustmaß dP' in Abhängigkeit vom Gefäßquerschnitt $A$

\section{Schlussfolgerung}

1. Eine Durchblutungssteigerung der Wirbelarterie wird bis wenig oberhalb des mittleren Lumendurchmessers von 3,1 mm sowohl durch eine Weitstellung des Gefäßes als auch eine Strömungsbeschleunigung realisiert. Eine Durchblutungssteigerung darüber hinaus wird ausschließlich durch Weitstellung der Wirbelarterie bedingt.

2. Der enddiastolische Blutdruckverlust in der Wirbelarterie durch strömungsbedingte Reibung sinkt mit zunehmender Durchblutung. Mit anderen Worten wird bei zunehmender „Transitfunktion“ der Wirbelarterie Strömungsenergie für a.e. nachgeschaltete Strömungsbereiche aufgespart.

\section{P2.007 Bestimmung von Flächenwerten eines Nomogramms der ACl und ACC mittels B-Flow Technik}

Autoren Ausmeier $\mathrm{A}^{1}$, Weskott $\mathrm{HP}^{2}$

Institut 1 Nordstadt Klinikum, Hannover/DE; 2 Klinikum Siloah, Hannover/DE DOI 10.1055/s-0037-1606984

Problemstellung Evaluation alters- und geschlechtsbezogener Querschnittsflächenwerte mittels B-Flow-Technik für die $\mathrm{ACl}$ und $\mathrm{ACC}$ in einer gesunden Stichprobe.

Patienten und Methode Messung der morphologischen Flächenwerte der ACC und $\mathrm{ACl}$ als Referenzgefäß hinter dem Bulbus caroticus bei 272 gesunden Probanden zwischen 22 und 90 Jahren (133 Männer, 139 Frauen) mithilfe der B-Flow Sonografie bei einem Winkel von $90^{\circ}$.

US Gerät: LOGiQ E9,9L (GE Healthcare, Milwaukee, IL, USA).

Ergebnis Das Durchschnittsalter in der Gruppe der gesunden Probanden betrug 59,1+16,9 Jahre bei den Männern und 51,5+16,4 Jahre bei den Frauen. Die Flächenwerte für die LACC, RACC, LACI und RACI betrugen bei den Män- 
nern: $38,6 \mathrm{~mm}^{2}+6,7 \mathrm{~mm}^{2}, 39,1 \mathrm{~mm}^{2}+6,2 \mathrm{~mm}^{2}, 22,3 \mathrm{~mm}^{2}+2,4 \mathrm{~mm}^{2}$, $22,6 \mathrm{~mm}^{2}+2,5 \mathrm{~mm}^{2}$, bei den Frauen: $30,8 \mathrm{~mm}^{2}+5,7 \mathrm{~mm}^{2}, 31,9 \mathrm{~mm}^{2}+$ $5,8 \mathrm{~mm}^{2}, 17,7 \mathrm{~mm}^{2}+2,9 \mathrm{~mm}^{2}, 18,0 \mathrm{~mm}^{2}+3,1 \mathrm{~mm}^{2}$.

Es zeigte sich eine mit zunehmendem Alter leicht ansteigende Tendenz der Flächenwerte (Männer $r=0,38 \mathrm{ACC}, r=0,12 \mathrm{ACl}$; Frauen $r=0,32 \mathrm{ACC}, r=0,17$

$\mathrm{ACl}$ ) und ein hochsignifikanter Unterschied zwischen den Geschlechtern in jeder Altersdekade ( $\mathrm{p}<0,1 \% \mathrm{ACl}$ und $\mathrm{ACC})$.

Schlussfolgerung Die Flächen des Gefäßlumens für die ACC und ACI können verlässlich, mit einer geringen Standardabweichung, berechnet werden. Über alle Altersdekaden fanden sich signifikant größere Flächenwerte der ACC und ACl zwischen den Geschlechtern.

Hohe Carotisbifurkationen, gewundene Gefäßverläufe, Kinking und Kalzifikationen sind die Hauptlimitationen dieser Technik.

Die B-Flow-Sonografie ist ein geeignetes Verfahren, um verlässlich Flächenwerte für die ACC und ACl zu berechnen. Es ist eine Ergänzung zum Dopplerverfahren, um Aussagen über morphologische Werte zu liefern, die z.B. bei nicht zu beurteilendem Referenzgefäß zur Beurteilung von Stenosegraden herangezogen werden können.

\section{P2.008 Zusammenfassung der wissenschaftlichen Erkenntnisse aus 10 Jahren Ultraschall-basierter Diagnostik und konservativer Therapie von Hüftdysplasie in der Mongolei}

\author{
Autoren Baumann $\mathrm{T}^{1}$, Munkuu $\mathrm{B}^{2}$, Essig $\mathrm{S}^{1}$, Schmid $\mathrm{R}^{3}$ \\ Institut 1 Institut für Hausarztmedizin und Community Care, Luzern/CH; \\ 2 National Center for Maternal and Child Health, Ulaanbaatar/MN; 3 Baarer \\ Kinderarztpraxis, Baar/CH \\ DOI 10.1055/s-0037-1606985
}

Problemstellung Die Hüftdysplasie (DDH) ist die häufigste Entwicklungsstörung des Bewegungsapparats und führt, wenn falsch oder ungenügend behandelt, zu einem lebenslangen Leiden. So auch in der Mongolei. Seit zehn Jahren wird dort, in Zusammenarbeit mit der Regierung, ein Neugeborenscreening für DDH im ganzen Land aufgebaut. Neben der Ausrüstung mit Ultraschallgeräten wurden die mongolischen Ärztinnen in der Methode Graf ausgebildet und durch ein webbasiertes Qualitätskontrolltool unterstützt.

Patienten und Methode 2007 wurde nach Einführung der Graf-Methode in Ulaanbaatar eine erste Studie durchgeführt. 572 Kinder zeigten die aus dem deutschsprachigen Raum bekannte Inzidenz der Hüfttypen. Es entsteht ein landesweites Screeningprogramm, welches erlaubt in einer prospektiven Kohortenstudie eine wachsende Zahl von Kindern in immer mehr Landesregionen zu erfassen. Dabei werden alle Kinder mit DDH mittels Tübingerschiene behandelt.

Ergebnis Durch die einfache Behandlungsmethode, die in der Regel in den ersten Lebenstagen begann, konnten alle Kinder im Schnitt nach 6 Wochen geheilt entlassen werden (Munkhuu et al. 2013). Die Untersuchungen werden im Internet überwacht (4-Augen-Prinzip) und erreichen eine hohe Qualität. Eine Nachkontrolluntersuchung der behandelten Kinder im Alter von 4 Jahren ergab einen bleibenden Behandlungserfolg ohne Sekundärschäden in Form von Hüftkopfnekrosen oder residuellen Dysplasien (Essig et al., under review). Nach nun zehn Jahren können die Neugeborenen aller Regionen der Mongolei (rund 80'000 Geburten/Jahr) untersucht und allenfalls behandelt werden. Schlussfolgerung Das diagnostische und konservativ-therapeutische Vorgehen wurde vereinfacht und es konnte dessen Wirksamkeit nachgewiesen werden. Ein Präventionsprogramm für Hüftdysplasie kann mit einfachen Mitteln erreicht und nachhaltig weiterentwickelt werden. Eine Kosten-Nutzen-Studie zur Übertragbarkeit auf ein Land wie die Schweiz ist in Vorbereitung.

\section{P2.009 Das Überziehen der vaginalen Ultraschallsonden mit Schutzhüllen - Risiko für nosokomiale Infektionen?}

Autoren Takacs $F Z^{1}$, Thieme-Ruffing $S^{2}$, Barbara $G^{2}$, Hamza $A^{1}$, MeybergSolomayer $\mathrm{GC}^{1}$

Institut 1 Universität des Saarlandes, Homburg/DE; 2 Universitätsklinikum des Saarlandes und Medizinische Fakultät der Universität des Saarlandes,

Homburg/DE

DOI 10.1055/s-0037-1606986

Problemstellung Ziel der Studie war es zu untersuchen, ob durch die Vorbereitung der Vaginalsonografie, trotz adäquater Aufbereitung der Sonden, die Möglichkeit einer iatrogenen Übertragung von Infektionen besteht.

Patienten und Methode Nach Überziehen der Vaginalsonde mit einer Schutzhülle wurden Abklatsche durchgeführt. Die einzelnen Schritte des Überziehens wurden dabei modifiziert, wodurch wir deren Auswirkung auf die Bakterienbesiedlung der Schutzhülle festhalten konnten.

Die Entnahme von nicht einzelverpackten Schutzhüllen aus einem offenen Spender wurde in einer zweiten Versuchsreihe durch serielle Abklatsche untersucht.

Ergebnis Nach Aufrollen der Schutzhülle auf die Vaginalsonde ohne vorherige Händedesinfektion und ohne keimarme Einmalhandschuhe war eine bakterielle Kontamination nachweisbar. Nach hygienischer Händedesinfektion (ohne Einmalhandschuhe), sowie nach Benutzung von unsterilen Einmalhandschuhen zum Aufrollen der Hülle, war dies nicht der Fall.

Beim ersten Öffnen des Hüllenspenders konnte keine bakterielle Kontamination gezeigt werden. Nach 3 Tagen war bereits ein Keimwachstum im Spenderkarton nachweisbar. Die Keimbelastung nahm in den Folgeuntersuchungen zu.

Schlussfolgerung Beim Überziehen der Sonde mit nicht desinfizierten Händen oder ohne Einmalhandschuhe konnte eine bakterielle Kontamination der Schutzhülle nachgewiesen werden. Dieser Arbeitsschritt der Untersuchung könnte daher ein Risiko für eine Übertragung nosokomialer Infektionen sein. Bei offenen Schutzhüllenspendern steigt das Risiko einer bakteriellen Kontamination der Schutzhüllen mit der Zahl der Entnahmen. Unter den experimentellen Bedingungen worden keine multiresistente Erreger detektiert, eine Kontamination ist jedoch im klinischen Alltag nicht komplett auszuschließen. Resultierend aus dieser Studie wurde unserer Hygienestandard dahingehend angepasst, dass vor dem Überziehen von Sondenschutzhüllen eine Händedesinfektion erfolgen muss oder keimarme Einmalhandschuhe angezogen werden müssen. Außerdem kommen ausschließlich einzeln verpackte Schutzhüllen zum Einsatz.

\section{P2.010 Verbesserung theoretischer und praktischer Fertigkeiten durch zertifizierte Ultraschall- Ausbildungskurse in der Anästhesie}

\section{Autoren Stark $\mathrm{K}^{1}$, Scheiermann $\mathrm{P}^{1}$, Tomasi $\mathrm{R}^{1}$}

Institut 1 Universitätsklinik LMU München, München/DE

DOI 10.1055/s-0037-1606987

Problemstellung Da der Einsatz von Ultraschall Komplikation vermeidet, wird Ultraschall auch zunehmend in der Anästhesie und Intensivmedizin eingesetzt. Zu Ausbildungszwecken wurde das modulare Konzept der Anästhesie-fokussierten Sonografie (AFS) von der Deutschen Gesellschaft für Anästhesiologie und Intensivmedizin (DGAI) etabliert. Da eine wissenschaftliche Validierung der 5 AFS-Module bislang noch nicht erfolgt ist, sollte untersucht werden, ob die Teilnehmer DGAI-zertifizierter Ultraschallkurse einen theoretischen und praktischen Wissenszuwachs erfahren.

Patienten und Methode Alle AFS-Module wurden an insgesamt vier Tagen an der Klinik für Anästhesiologie am Klinikum der LMU München durchgeführt. 20 Teilnehmer absolvierten für alle Module jeweils einen Prä- sowie einen Postkurstest mit praktischen und theoretischen Aufgabestellungen. Die erho- 
benen Daten wurden mithilfe des Mann-Whitney-Test, sowie dem paired-TTest analysiert.

Ergebnis Im Kurs über die Grundlagen, Gefäß- und Neurosonografie verbesserten sich die Teilnehmer nach dem Kursbesuch im theoretischen Test um 19,2\% ( $p<0,05$ ). Bei den praktischen Übungen konnten nach dem Kursbesuch Bilder von vorgegebenen Strukturen signifikant schneller $(p=0,03)$ dargestellt, anatomische Strukturen aber nicht detaillierter $(p=0,52)$ benannt werden. Im Kurs über die Kardiosonografie und Thorakoabdominelle Sonografie verbesserten sich die Teilnehmer sowohl im theoretischen Test, wie auch in der Diagnosestellung von Krankheitsbildern ( $p=0,0079)$.

Schlussfolgerung Die Studie zeigt, dass das AFS-Kursformat vor allem die theoretischen bzw. diagnostischen Kenntnisse der Teilnehmer verbessert. In den praktischen Fertigkeiten konnten wir trotz zahlreicher Übungsstationen nur begrenzte Verbesserungen aufzeigen. Daher muss der praktische Wissenszuwachs in diesem Kursformat durch geeignete Strategien deutlich mehr gefördert werden.

\section{P2.011 Optimierung des Strain-Elastografie durch Computer assistierte Steuerung der Ultraschallsonde}

Autoren Bauermeister $\mathrm{W}^{1}$

Institut 1 Schmerzinstitut, München/DE

DOI 10.1055/s-0037-1606988

Problemstellung Das Ergebnis der Strain-Elastografie ist weitgehend vom Bediener abhängig. Die Erzeugung des Strain mit der freien Hand kann durch unterschiedlichen Anpressdruck, Druckfrequenz und Druckverteilung des Schallkopfes sehr variable Befunde ergeben. Wenn diese Parameter genau definiert und konstant gehalten werden, könnte es möglich sein, Ergebnisse zu erzielen, die vom Bediener weitgehend unabhängig sin.

Patienten und Methode Zur Erzeugung des Strains wird ein Vibrationsgenerator (Frederiksen no. 2185.00, Frequenzbereich $0,1-5 \mathrm{kHz}$, Maximalamplitude $7 \mathrm{~mm}$ bei $1 \mathrm{~Hz}$ ) eingesetzt. Zur Steuerung der Strain-Frequenz und StrainAmplitude wird ein Funktionsgenerator mit Leistungsausgang (Function Generator Type CL 01187, Signalform: Sinus, Rechteck, positives Rechteck, Frequenzbereich $0,05 \mathrm{~Hz}$ bis $50 \mathrm{kHz}$ ) verwendet. Durch eine zangenförmige Metallhalterung wird der lineare Ultraschallkopf $\mathrm{L}$ 14-5 W mit einer Breite von $60 \mathrm{~mm}$ (SonixOne Analogic Ultrasound USA) mit dem Vibrationsgenerator verbunden. Die Ultraschallsonde wird mittels des B-Bildes im Ruhezustand so positioniert um einen Kontakt mit der Haut zu erreichen ohne das Gewebe zu deformieren. Nach Einschalten des Vibrationsgenerators wird die Bewegung des Gewebes kontrolliert, um sicherzustellen, dass alle Schichten von dem Strain erfasst werden. Der Funktionsgenerator wird auf die optimale Frequenz zwischen 2-4 Hz eingestellt, um ein stehendes sich ständig wiederholende Elastografie-Bild zu erzeugen.

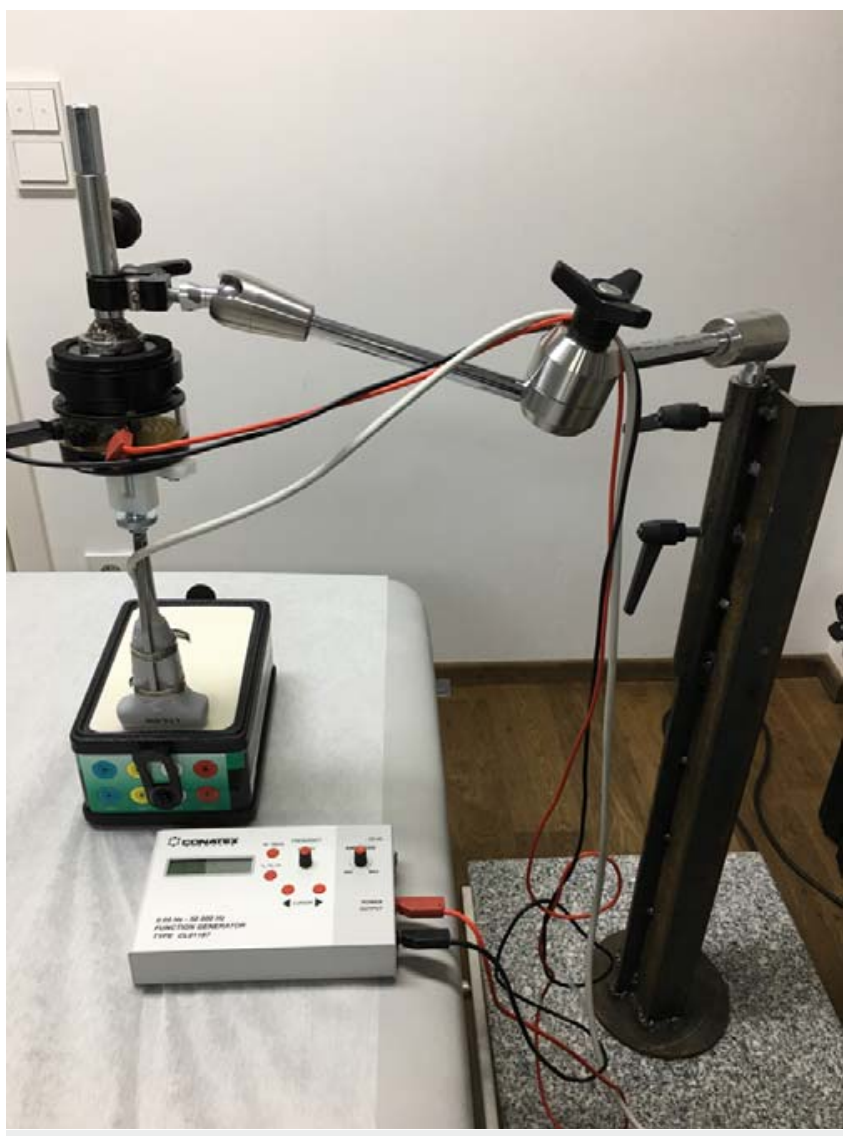

Abb. 1 Gelenkarm mit Vibrationsgenerator

Ergebnis Mit diesem Versuchsaufbau liegt die optimale Strain-Frequenz zwischen 2-4 Hz bei einer Amplitude von ca. 3- $7 \mathrm{~mm}$.

Schlussfolgerung Die Strain-Erzeugung mit einem Vibrationsgenerator ermöglicht es die Elastografie unter konstant gehaltenen Bedingungen durchzuführen. Dieses verbessert die Reproduzierbarkeit der Untersuchungsergebnisse und schafft die Möglichkeit auch für unerfahrene Untersucher gute Ergebnisse zu erzielen.

\section{P2.012 Vergleich von quantitativer monochromem Superb-Microvascular-Imaging (mSMI) mittels Image] und Kontrastverstärkter Sonografie (VueBox) bei Lebermetastasen - eine Pilotstudie}

Autoren Dobler $\mathrm{F}^{1}$, Kratzer $\mathrm{W}^{2}$, Schmidberger J ${ }^{1}$, Gräter $\mathrm{T}^{1}$, Hänle $\mathrm{M}^{1}$, Pfahler $\mathrm{M}^{1}$, Grüner $\mathrm{B}^{1}$

Institut 1 Universitätsklinikum Ulm, Ulm/DE; 2 Uniklinik Ulm, Ulm/DE DOI 10.1055/s-0037-1606989

Problemstellung Evaluation der diagnostischen Wertigkeit der quantitativer monochromem Superb-Microvascular-Imaging (mSMI) mittels ImageJ im Vergleich mit Kontrastmittelverstärktem Ultraschall (CEUS) VueBox und Histologie.

Patienten und Methode Es wurden dreizehn Patienten mit gastrointestinalen oder pankreatikobiliären Lebermetastasen mithilfe von mSMI quantitativ, CEUS quantitativ und Histologie untersucht. Dabei wurden die mSMI-Ergebnisse mittels Grauwertanalyse durch das Bildanalyseprogramm Imagej als Vaskularisierungsquotient (VQ) quantifiziert und mit den durch VueBox ${ }^{\circledR}$ errechneten Werten der CEUS-Untersuchung verglichen.

Ergebnis Der Vaskularisierungsquotient (VQ) zeigte in allen Fällen ein im Vergleich zum normalen Leberparenchym stärkeres mSMI-Signal $(p=0,0002)$. 
Zwischen dem VQ und den quantifizierten CEUS-Werten besteht eine wertebezogene Korrelation von $r=0,31$ bis $r=0,83$.

Schlussfolgerung Mithilfe von mSMI quantitativ und dessen Quantifizierung durch ImageJ kann der Durchblutungszustand von fokalen Leberläsionen (FLL) beurteilt werden. Dies könnte zukünftig bei der Dignitätsbeurteilung von Lebertumoren hilfreich sein.

\section{P2.013 Die Rolle des Ultraschalls bei der Kaudalanästhesie}

Autoren Santamaria $\mathrm{M}^{1}$, Erker $\mathrm{C}^{1}$, Möllmann $\mathrm{M}^{2}$

Institut 1 St. Franziskus Hospital Münster, Münster/DE; 2 St. Franziskus

Hospital Münster, Münser/DE

DOI 10.1055/s-0037-1606990

Problemstellung Der Einsatz von Ultraschall bei regionalanästhesiologischen Verfahren erfährt in den letzten Jahren eine zunehmende Verbreitung und Akzeptanz. Prognostisch werden Ultraschallgeräte in wenigen Jahren zur Grundausstattung von Anästhesiearbeitsplätzen gehören.

Patienten und Methode Auch im Bereich der Kinderanästhesie ergeben sich mit der Etablierung von Ultraschallgeräten interessante Anwendungsmöglichkeiten. Klassische und häufig nach Landmarkentechnik durchgeführte Verfahren wie die Kaudalanästhesie können von den technischen Möglichkeiten der Visualisierung der Prozedur mittels Ultraschall profitieren.

Ergebnis So kann z.B. durch eine orientierende sonografische Untersuchung vor der Punktion eine eventuelle Fehlbildung im Bereich des Hiatus sacralis ausgeschlossen werden. Die Nadel kann sonografisch dargestellt und das korrekte Anfluten des Lokalanästhetikums im Periduralraum beobachtet und bestätigt werden.

Schlussfolgerung Somit erlangt man durch das Verfahren eine größere Sicherheit im Hinblick auf die grundsätzliche Möglichkeit der Punktion, der korrekten Injektion und auch bezüglich einer genauen Aussage über den Erfolg der Blockade. Dies ist insbesondere dann von Vorteil, wenn der Kaudalblock als reines Regionalanästhesieverfahren durchgeführt wird. Darüber hinaus kann es aus didaktischen Gründen sinnvoll sein, die Ausbreitung des Lokalanästhetikums zu visualisieren, um ein Gefühl für die Punktion und die Injektion zu entwickeln.

Literatur [1] Koscielniak-Nielsen Z] (2008): Ultrasound peripheral nerve blocks: what are the benefits? Acta Anaesthesiol Scand; 52 (6): 727 - 737

[2] Mauch J, Weiss M (2012): Kaudalanästhesie bei Kindern. Der Schmerz; 16

(4): $443-454$

[3] Jöhr M, Berger TM (2012): Caudal blocks. Paediatr Anaesth 22: 44 - 50

[4] Hillmann R, Döffert J (2009): Praxis der anästhesiologischen Sonografie. Urban \& Fischer, 1. Auflage

\section{P2.014 Lumbar puncture with Ultrasound Study (LUPUS Study) - International Prospective Randomized Multicentre Trial}

Authors Vosko MR ${ }^{1}$, Brunner $C^{2}$, Schreiber $S^{3}$

Institute 1 Kepler Universitätsklinikum, Linz/AT; 2 Kepler

Universitätsklinikum, Med Campus III, Linz/AT; 3 ASKLEPIOS Fachkliniken, Brandenburg/DE

DOI 10.1055/s-0037-1606991

Problemstellung Lumbar puncture is a common procedure used to obtain information about the cerebrospinal fluid (CSF), but in obese patients still remains challenging. Ultrasound-guided lumbar puncture seems to be helpful in those patients in whom surface landmark-guided lumbar puncture is difficult.

Patienten und Methode LUPUS is an international prospective randomized multicentre trial comparing the ultrasound defined puncture site to palpatory and landmarks defined puncture site for a routine lumbar puncture in patients with body mass index (BMI) higher then 25. Using ultrasound (US), the lumbar spine anatomy is visualized in transverse and longitudinal planes and the needle insertion site is marked.

The hypothesis is, that ultrasound enhances the effectiveness of lumbar puncture. Primary endpoints are: number of puncture attempts by one patient and number of re-directions. Inclusion criteria: patients indicated for lumbar puncture with $\mathrm{BMI}>25$, consecutive randomisation in the group with palpatory definition or in the group with US definition of the puncture site.

Ergebnis 220 Patients with BMI of $31 \pm 5$ were divided into palpatory $(\mathrm{N}=113)$ and US groups $(\mathrm{N}=107)$. There was a statistically relevant difference in number of punctions (1.6 vs. $1.5 ; \mathrm{p}<0.02)$ as well as in number of redirections (6.7 vs. 5.2; $p<0.0017)$.

Schlussfolgerung Ultrasound defined puncture site for lumbar puncture reduces the number of puncture attempts and number of re-directions.

\section{P2.015 Evaluation of complex renal cystic lesions with contrast enhanced ultrasound (CEUS) and functional MRI versus the gold standard: computer tomography (CT)}

Authors Arampatzis $\mathrm{S}^{1}$, Kiss $\mathrm{B}^{1}$, Tshering Vogel $\mathrm{D}^{1}$, Burkhard $\mathrm{F}^{1}$,

Heverhagen $]^{1}$, Vogt $B^{1}$

Institute 1 Inselspital, Bern/CH

DOI 10.1055/s-0037-1606992

Problemstellung The primar aim of this study was to assess and compare the diagnostic performance of contrast enhanced ultrasound (CEUS) and magnetic resonance imaging (MRI) with computer tomography (CT) for classification of cystic renal lesions according to the Bosniak system.

Patienten und Methode This prospective observational study, starting from July 2014, has been approved by the ethic committee. 28 patients ( 9 women and 19 men; age range 36-88 years; median age 57years) with complex cystic lesions of the kidney were scanned on an Acuson $\$ 3000$ ultrasound scanner (Siemens) after injection of SonoVue and in a 3Tesla MRI machine, Skyra (Siemens). CT scan was performed during routine clinical workup.

Ergebnis On CT scans, the lesions were classified as Bosniak I $(n=1)$, Bosnaik II $(n=9)$, Bosniak IIf $(n=4)$, Bosniak III $(n=3)$ and Bosniak IV $(n=11)$. Similar evaluation on MRI resulted in Bosniak I $(n=1)$, Bosnaik II $(n=12)$, Bosniak IIf $(n=1)$, Bosniak III $(n=4)$ and Bosniak IV $(n=10)$ and on CEUS as Bosniak I $(n=0)$, Bosnaik II $(n=2)$, Bosniak IIf $(n=9)$, Bosniak III $(n=5)$ and Bosniak IV $(n=12)$.

MRI showed a better correlation with CT $(24 / 28)$ than CEUS. CEUS overestimated the Bosniak grade in 11/28 patients. One Bosniak IV lesion on CT was classified as Bosniak III on both MRI and CEUS due to better visualization of the multiple septae which appeared more solid on CT.

Schlussfolgerung MRI classification of Bosniak cysts correlated better with the $\mathrm{CT}$ classification. CEUS tended to upgrade the classification due to better spatial resolution and better visualization of the enhancement.

\section{P 3: Poster Gyn/Geburtshilfe Poster Präsentation}

\section{P3.001 Fetale Gastroschisis: pränatale Diagnostik - neonatales Outcome}

Autoren Fröhlich $C^{1}$, Festl $]^{2}$, Wöckel $A^{2}$, Rehn $M^{2}$

Institut 1 Unifrauenklinik Würzburg, Würzbrug/DE; 2 Uniklinik Würzburg, Würzburg/DE

DOI 10.1055/s-0037-1606993

Patienten und Methode Eine pränatale Diagnose und Überwachung einer fetalen Gastroschisis ist ausschlaggebend für ein gutes neonatales Outcome. Anhand verschiedener Ultraschallparameter ist die weitere pränatale Überwachung vorzunehmen. Im Falle einer Darmdilatation kommt dem eine entscheidende Bedeutung zu. Diese ist vergesellschaftet mit einer erhöhten Rate an 
postnatalen Darmkomplikationen. 2 Fälle einer pränatal diagnostizierten fetalen Gastoschisis mit intraabdominaler Darmdilatation sollen im Kontext zu dem postnatalen Outcome vorgestellt werden.

Methodik: 2 Patientinnen wurden mit der Diagnose einer fetalen Gastroschisis an unserem Perinatalzentrum überwacht. Bei einer Patientin bestand ein Opiat- und Nikotinabusus während der gesamten Schwangerschaft. Engmaschige sonografische Kontrollen erfolgten. In der 33. bzw. 34 SSW deutliche Zunahme der Darmdilatation, Entbindungsmodus ${ }^{\circ}$ Sectio.

Ergebnisse: Im Falle des Neugeborenen der polytoxikomanen Mutter zeigte sich ein komplexes abdominales Fehlbildungssyndrom mit Gastroschisis, Jejunalatresie, Dünndarmatresie Typ IIlb sowie eine Kolonstenose und ein funktionelles Kurzdarmsyndrom. Bis zum Alter von 3 Monaten mussten 4 Operationen, unter anderem mit Anlage multipler Stomata, durchgeführt werden. Im Falle der zweiten Patientin zeigte das Kind postnatal eine vollständige Nekrose des intrauterin extraabdominal gelegenen Dünndarms. Auch hier wurden in den ersten Lebensmonaten 4 Operationen durchgeführt.

Schlussfolgerung In beiden Fällen handelt es sich um schwere postnatale Darmkomplikationen bei pränatal diagnostizierter fetaler Gastroschisis mit intra- und extraabdominaler Darmdilatation. Eine pränatal diagnostizierte Darmdilatation ist assoziiert mit einem postnatal komplikationsreicheren Verlauf. In wieweit in dem ersten Fall der maternale Drogenabusus das Outcome negativ beeinflusst, muss in der Diskussion berücksichtigt werden. Literatur [1] K. Lato, et al, Ultraschall in Med 2013; 34: 157 - 161

[2] A. Brantberg et al., Ultrasound Obstet Gynecol 2004; 23: 4-13

\section{P3.002 Chorangiom mit $11 \times 9 \times 6 \mathrm{~cm}$ Größe - perinatales Management}

Autoren Vogeler $\mathrm{F}^{1}$, Duwe $\mathrm{W}^{2}$, Dombrowski $\mathrm{F}^{3}$, Ruhland $\mathrm{F}^{1}$

Institut 1 gSund Kompetenzzentrum Gynäkologie, Stralsund/DE; 2 Helios Klinik Stralsund, Stralsund/DE; 3 Universitätsmedizin Greifswald, Greifswald/ DE

DOI 10.1055/s-0037-1606994

Patienten und Methode Mit einer Inzidenz von 0,6\% - 1\% gilt das Chorangiom als der häufigste Tumor der Plazenta. Arteriovenöse Anastomosen können fetale hämodynamische Komplikationen bewirken (sekundäre Herzinsuffizienz; Hydrops; IUFD; Anämie mit konsekutiver IUGR). Beschriebene maternale Komplikationen sind Hydramnion, vorzeitige Plazentalösung/Blasensprung, schwangerschaftsinduzierte Hypertonie; HELLP-Syndrom sowie postpartale Blutung.

Fallbericht Aufnahme einer 34-jährigen GII/PI in der 29+3. SSW mit vorzeitiger Wehentätigkeit und Polyhydramnion.

Sonografie: vitale Einlingsgravidität $1736 \mathrm{~g}, \mathrm{AFI} 44 \mathrm{~cm}$, einem Chorangiom entsprechender $11 \mathrm{~cm} \times 6 \mathrm{~cm} \times 9 \mathrm{~cm}$ inhomogener, vaskularisierter Tumor der Vorderwandplazenta (Abb. 1a-1c). Zunächst Lungenreifeinduktion unter i.v.-Bolus-Tokolyse. Bei Größenkonstanz des Plazentatumors (Abb. 2), Regredienz des Hydramnions und unauffälligen Dopplerparametern Fortführung des konservativen Managements ab 32. SSW im ambulanten Setting.

Beendigung der Schwangerschaft in der 37. SSW per elektiver Sectio (3020 g; APGAR 9/10/10; NapH:7,33; keine neonatalen Anämiezeichen).

Histologie: Chorangiom bei altersentsprechender ausgereifter Plazenta.

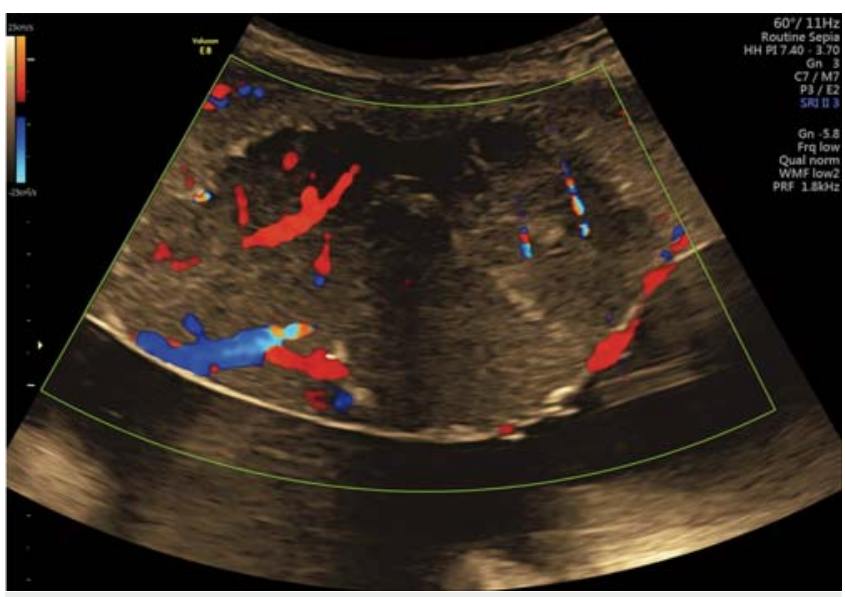

Abb. 1a Chorangiom

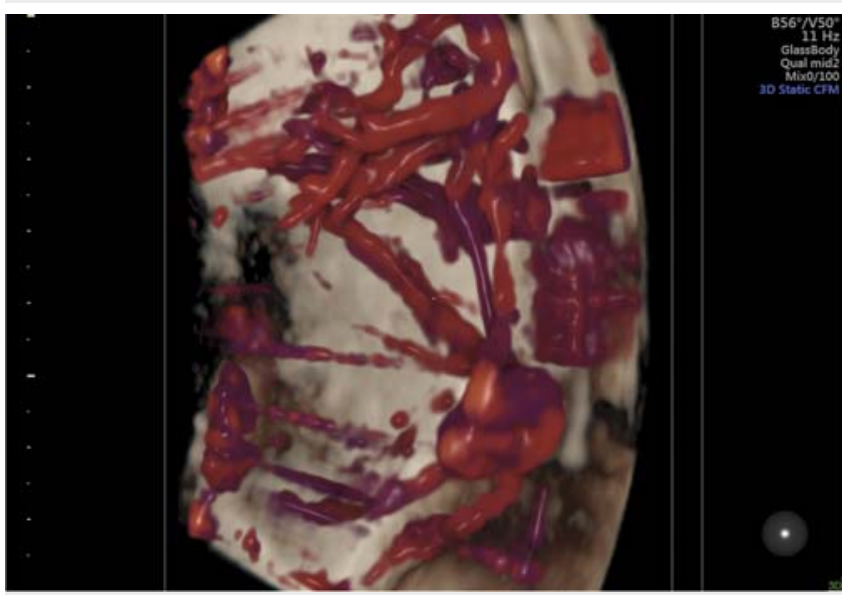

Abb. 1c Chorangiom

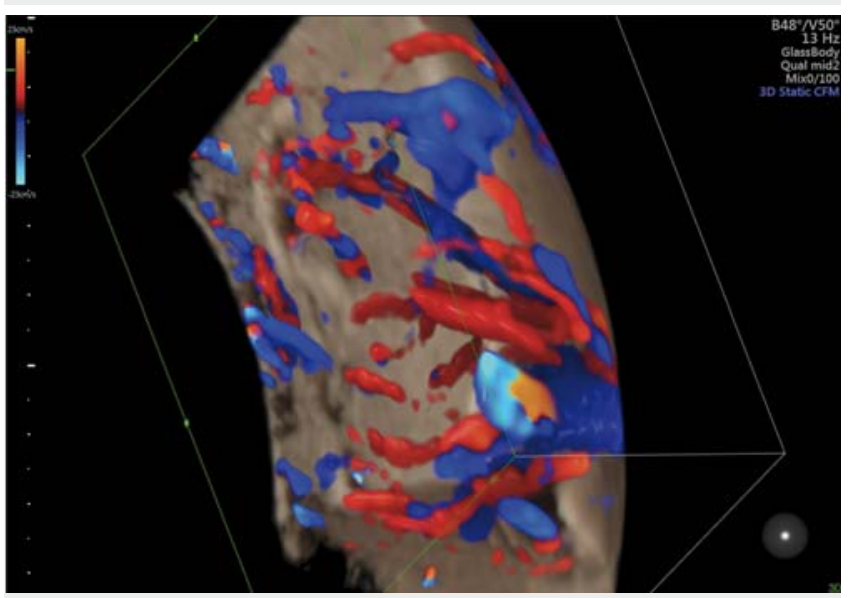

Abb. 2 Chorangiom

Schlussfolgerung In der Literatur wird die perinatale Mortalität mit 10\% (Sirotkina $M$ ) - 33\% (Lutgardo G.) angegeben sowie die konforme Empfehlung zur engmaschigen sonografischen Surveillance. In 2/3 der Fälle die primäre Sectio als Entbindungsmodus gewählt.

Kein Konsens besteht derzeit bzgl. dem perinatalem Management: invasive in utero Therapie vs. konservativem Vorgehen. Nach Entezami M. haben die wenigsten Chorangiome eine pathophysiologische Relevanz. Auf Basis der derzeitigen Studienlage lassen sich laut Vaidyanathan G. keine Selektionskriterien für das Verlassen des konservativen Regimes definieren. Für Leeb K. bleiben invasive Therapieoptionen experimentell. 
Dieser Fallbericht widerlegt das große, stark vaskularisierte Chorangiom zwangsläufig eine fetale kardiale Dekompensation bewirken und bestätigt den konservativen Therapieansatz.

\section{P3.003 Monoamniale Zwillingsschwangerschaften - Betreuung und Ausgang von Hochrisikoschwangerschaften}

\author{
Autoren Geiler $\mathrm{S}^{1}$, Riße $\mathrm{S}^{1}$, Stepan $\mathrm{H}^{2}$, Faber $\mathrm{R}^{1}$ \\ Institut 1 Zentrum für Pränatale Medizin, Leipzig/DE; 2 Universitätsklinik \\ Leipzig, Leipzig/DE \\ DOI 10.1055/s-0037-1606995
}

Problemstellung Monoamniale Geminigraviditäten treten selten auf und sind aufgrund der fetalen Gefährdung durch Nabelschnurverschlingungen, Frühgeburt und angeborenen Anomalien Hochrisikoschwangerschaften. Jüngere Daten zeigen, dass der perinatale Ausgang dennoch sehr gut ist, wenn die Schwangerschaften bis zur 20. Schwangerschaftswoche komplikationslos verlaufen. Die nachgewiesenen Nabelschnurverschlingungen stellen dabei keinen Grund für eine erhöhte Mortalität dar. Diese beruhen eher auf angeborenen Anomalien, TRAP-sequenzen oder spontanen Fehlgeburten in frühen Schwangerschaftswochen.

Patienten und Methode Im Zeitraum von 2007-2015 wurden im Zentrum für Pränatale Medizin Leipzig alle Schwangerschaften $(n=13)$ einbezogen, welche sonografisch, klinisch oder pathologisch anatomisch eindeutig als monoamnial definiert wurden.

Ergebnis Aufgrund von schwerwiegenden angeborenen Anomalien $(n=4)$, IUFT $(n=1)$ und TOP $(n=1)$ endeten 6 Schwangerschaften bereits von der 15. SSW. Eine Gravidität mit TRAP-sequenz wurde extern mit Lasertherapie behandelt und endete mit einem gesunden Mehrling in der 32+4 SSW. Bei den anderen 6 Schwangerschaften, welche bis zur 20. SSW komplikationslos verliefen, zeigte sich trotz sonografisch nachgewiesener Nabelschnurverschlingung keine Mortalität. Das mittlere Gestationsalter dieser 13 Feten betrug bei Entbindung $33+4$ SSW $(32+2$ SSW bis $36+0$ SSW) und das durchschnittliche Geburtsgewicht 2053 Gramm (1630 Gramm bis 2700 Gramm).

Schlussfolgerung Wir können die Ergebnisse der Literatur bestätigen, dass bei fehlenden Komplikationen bis zur 20. SSW trotz bestehenden Nabelschnurverschlingungen eine sehr geringe bis keine Mortalität zu erwarten ist. Bei hochspezialisierter Betreuung ist deshalb eine lange ambulante Überwachung gerechtfertigt.

\section{P3.004 Einführung eines praktischen Ultraschall- und Laparoskopiekurs in der Anatomielehre am UKS}

Autoren Hamza A $\mathrm{A}^{1}$, Meyberg-Solomayer $\mathrm{GC}^{1}$, Solomayer $\mathrm{EF}^{1}$, Takacs $Z^{1}$, Juhasz-Boess $I^{1}$, Radosa $J^{1}$, Ströder $R^{1}$, Tschernig $T^{1}$, Maxeiner $S^{1}$

Institut 1 Universität des Saarlandes, Homburg/DE

DOI 10.1055/s-0037-1606996

Problemstellung Die Anatomie ist ein Grundstein in der Medizinlehre. Aufgrund der zunehmenden Neuigkeiten in der Medizin wurden im Verlauf der Jahre viele Fächer neu eingeführt, dafür aber die Lehrzeit u. a. im Fach Anatomie gekürzt. Daneben bestehen seit Jahren ideale und genaue bildgebende diagnostische Verfahren, u.a. Laparoskopie und Ultraschall, die in der Grundlehre nicht optimal eingeführt wurde. In unserer Arbeit untersuchten wir die Auswirkungen der Anwendung standardisierter gynäkologischer Ultraschallund Laparoskopielehre in der Frauenklinik bei Studenten im Anatomiekurs.

Patienten und Methode Anatomiestundenten haben nach abgeschlossenen konventionellen Anatomiekurs (Leichendissektion und Vorlesungen) an einen theoretischen und praktischen Ultraschall- oder Laparoskopiekurs erhalten. In beiden Kursen wurden Organe des weiblichen Beckens in Anwesenheit der Studenten dargestellt und erklärt. Im Anschluss an die Kurse erfolgte eine Evaluation dieses Kurses durch 2 Fragebögen. Im ersten Fragebogen mussten die Studenten 11, im zweiten 16 Fragen beantworten. Es wurden die allge- meine Zufriedenheit, Probleme, subjektive Wissenserweiterung und Qualität des Kurses mittels Likert-Score bewertet.

Ergebnis 25 Studenten haben am Kurs teilgenommen (52\% Laparoskopie-, $48 \%$ Ultraschallgruppe). Aus dem Evaluationsbogen ergab sich eine allgemeine Zufriedenheit der Studenten (Likert 1,5), bei knapp über der Hälfte subjektiv die Einschätzung einer Wissenserweiterung (Likert 2,4) und die Forderung nach mehr sonografischem und laparoskopischem Hands-on im Gebiet der Frauenheilkunde (Likert 1,5) sowie in anderen Bereichen der Medizin (Likert 1,2). Zwischen der Laparoskopie- und Ultraschallgruppe ergaben sich keine statistisch signifikanten Unterschiede.

Schlussfolgerung Die Einführung von Ultraschall- und Laparoskopielehre in die Anatomielehre ist eine versprechende Methode und sollte künftig vermehrt evaluiert, standardisiert und ausgeweitet werden.

\section{P3.005 Assoziation fetaler biometrischer Parameter mit dem perinatalen Outcome}

Autoren Pretscher $\mathrm{J}^{1}$, Raabe $\mathrm{E}^{1}$, Baier $\mathrm{F}^{1}$, Kehl $\mathrm{S}^{1}$, Schneider $\mathrm{M}^{1}$,

Stumpfe $\mathrm{FM}^{1}$, Schmid $\mathrm{M}^{2}$, Beckmann $\mathrm{M}^{1}$, Mayr $\mathrm{A}^{1}$, Schild $\mathrm{R}^{3}$,

Faschingbauer $\mathrm{F}^{1}$

Institut 1 Universität Erlangen, Erlangen/DE; 2 Universität Bonn, Bonn/DE;

3 Universität Hannover, Hannover/DE

DOI 10.1055/s-0037-1606997

Problemstellung Analyse des Zusammenhangs zwischen sonografisch gemessenem Kopfumfang, Bauchumfang und Gewicht mit Geburtsmodus und perinatalem Outcome.

Patienten und Methode In der retrospektiven Studie wurden bei Einlingsschwangerschaften $\geq 37$ SSW mit vorhandener Ultraschalluntersuchung innerhalb einer Woche vor Entbindung die Assoziation zwischen sonografisch gemessenem Kopfumfang, Bauchumfang und Gewicht und fetalem (5-min Apgar $<7, \mathrm{pH}<7,10$, postpartaler Verlegung in die Kinderklinik, Schulterdystokie) und maternalem (sekundäre Sectio caesarea oder vaginal-operativer Entbindung, höhergradigen Geburtsverletzungen, postpartale Hämorrhagie) Outcome mittels uni- und multivariater logistischer Regression untersucht.

Ergebnis 12572 Frauen wurden eingeschlossen: 8942 (71,13\%) entbanden spontan, $3486(27,73 \%)$ entbanden per Sectio caesarea oder vaginal-operativ (geburtshilfliche Intervention). In der multivariaten Analyse zeigte sich eine signifikante Assoziation für die Vorhersage einer geburtshiflichen Intervention durch den sonografischen Kopf- $(O R$ 1,110, $p<0,001)$ und Bauchumfang (OR $1,100, p=0,002$ ), wohingegen das fetale Schätzgewicht keinen signifikanten Zusammenhang zeigte. Eine Schulterdystokie trat in 73 Fällen (0,58\%) auf; die multivariate Analyse dieses Parameters zeigte eine signifikante Assoziation für den sonografischen Abdomenumfang ( $O R$ 1,468, $p=0,006$ ), wohingegen weder der sonografische Kopfumfang noch das Schätzgewicht einen signifikanten Einfluss ergaben. Für die anderen Outcome-Parameter zeigten sich keine signifikanten Zusammenhänge. Die Detektionsraten für die Prädiktion des adversen perinatalen Outcomes durch die erhobenen Parameter waren insgesamt niedrig.

Schlussfolgerung Entscheidungen über geburtshilfliches Management sollten nicht allein von der Erhebung sonografischer biometrischer Parameter oder dem Schätzgewicht abhängig gemacht werden.

\section{P3.006 Sonografische und invasive Pränataldiagnostik in Hessen von 2001 bis 2015}

Autoren Noever $\mathrm{K}^{1}$, Barkhaus $\mathrm{C}^{2}$, Timmesfeld $\mathrm{N}^{2}$, Misselwitz $\mathrm{B}^{3}$, Arabin $\mathrm{B}^{1}$ Institut 1 Philipps-Universität Marburg, Marburg/DE; 2 Philipps Universität Marburg, Marburg/DE; 3 Qualitätssicherung Hessen, Eschborn/DE DOI 10.1055/s-0037-1606998

Problemstellung Die Schwangerenbetreuung wird in Deutschland vorwiegend im ambulanten Bereich durchgeführt. Internationale Forschungsergebnisse haben hier zu grundlegenden Veränderungen geführt. Wir wollen unter- 
suchen, welche Veränderungen in der pränatalen Diagnostik sich über die Zeit zeigen.

Patienten und Methode Die Daten der Hessischen Perinatalerhebung von 2001-2015 umfassen 701708 Geburten darunter 688961 Einlings- und 12747 Mehrlingsschwangerschaften. Innerhalb dieses Kollektivs wurden pränatal und postnatal die Diagnose von Fehlbildungen nach der ICD-10-Klassifikation, Kapitel XVII verschlüsselt. Zusätzlich wurde die Durchführung von Chorionzottenbiopsien und von Amniozentesen erhoben.

Ergebnis Insgesamt stieg die Rate an pränatal diagnostizierten Fehlbildungen signifikant von 110 (0,24\%) im Jahr 2001 auf 206 (0,41\%) im Jahr 2015 an $(p<0,0001)$. Dagegen fiel die Rate an Chorionzottenbiopsien signifikant von $362(0,8 \%)$ auf $198(0,41 \%)$ im Jahr 2014 an $(p<0,0001)$. Ebenso verhielt es sich mit den Amniozentesen (Abfall von 12,35\% auf 2,89\%, $p<0,0001$ ).

Über den gesamten Zeitraum wurden 4194 (0,59\%) Kinder mit einer Fehlbildung geboren. Von diesen wurden bei $982(23,41 \%)$ bereits pränatal eine Fehlbildung diagnostiziert. Diese Rate nahm von 19,18\% im Jahr 2001 signifikant zu auf 30,25\% im Jahr 2015 ( $p=0,0002)$.

Insgesamt wurde bei 224047 (31,92\%) Geburten eine Sectio durchgeführt. Eine Fehlbildung als Indikation wurde in 1035 Fällen (0,46\%) angegeben.

Schlussfolgerung Die Analyse zeigt eine Zunahme der pränatal diagnostizierten Fehlbildungen, während die Durchführung der Amniozentese und Chorionzottenbiopsie abnahm. Dies muss jedoch im internationalen Vergleich beurteilt werden. Es wäre von hoher Wichtigkeit, das Ersttrimester-Screening und die zellfreie DNA-Diagnostik heute in populationsbezogene Erfassungsbögen aufzunehmen.

\section{P3.007 Isolierter fetaler Aszites: Korrelation des postnatalen Outcome mit pränatalen sonografischen Befunde}

Autoren Komar $\mathrm{M}^{1}$, Nitzsche $\mathrm{K}^{1}$, Birdir $\mathrm{C}^{1}$

Institut 1 Universitätsklinikum Carl Gustav Carus Dresden an der Technischen Unviersität Dresden, Dresden/DE

DOI 10.1055/s-0037-1606999

Problemstellung Der isolierte fetale Aszites ist als pathologische Ansammlung von Körperflüssigkeit in der fetalen Bauchhöhle definiert. Er stellt eine Rarität unter den pränatalen sonografischen Pathologien dar. Die Ätiologie des isolierten fetalen Aszites zeigt eine große Heterogenität. Ursachen können unter anderem chromosomale Aberrationen, intrauterine Infektionen, sowie Anomalien des Gastrointestinaltraktes sein. In ca. 4\% der Fälle kann keine Ursache gefunden werden.

Patienten und Methode Ziel dieser Arbeit ist Darstellung der prä- und postnatalen Befunde von 8 Patientinnen, die sich im Zeitraum von 2014 bis 2017 mit isoliertem fetalen Aszites in unserer Klinik vorstellten.

Ergebnis Die Erstdiagnose in den 8 Schwangerschaften wurde zwischen der $19+4$ bis $34+6$ SSW gestellt. In drei Fällen stellte sich zusätzlich ein Polyhydramnion dar. In einem Fall lag eine Cholestase aufgrund einer Hämatochromatose vor. Als weitere Ursachen fanden sich in einem Fall eine Duodenalstenose und in je zwei Fällen eine Trisomie21 und eine Mekoniumperitonitis. In zwei Fällen blieb die Ursache unklar. Von den 8 Neugeborenen wurde ein Kind operativ behandelt, ein Kind verstarb.

Schlussfolgerung Die Prognose hängt hauptsächlich von Begleiterkrankungen ab. Die Überlebensrate beträgt über $94 \%$. Neugeborene mit dieser Symptomatik haben meist ein besseres Outcome als Neugeborene mit einem generalisierten Hydrops fetalis. Zu den negativen prognostischen Faktoren gehören eine frühe Schwangerschaftswoche zum Zeitpunkt der Erstdiagnose und Grad der Ausprägung des Aszites.

\section{P3.008 Management einer Präeklampsie nach auffälligem Screening}

Autoren Becker $\mathrm{WH}^{1}$, Luths $\mathrm{A}^{2}$

Institut 1 Perinatalzentrum Altona, Hamburg/DE; 2 Städtisches Klinikum

Lüneburg, Lüneburg/DE

DOI 10.1055/s-0037-1607000

Patienten und Methode Die 29-jährige Erstgravida wurde unserem Zentrum in SSW 12+6 zum erweiterten Ersttrimesterscreening zugewiesen. Außer einem BMI von 38,2 fanden sich keine weiteren maternalen Risikofaktoren für Präeklampsie. Die Kalkulation für Trisomie 13/18/21 ergab unauffällige Werte, für Präeklampsie eher SSW 34+0 ergab sich 1:51 (Algorithmus der FMF UK inklusive PIGF). Wir verordneten ASS 100 jeweils abends bis SSW 34+0.

Bei persistierend erhöhtem uterinen Gefäßwiderstand und ansonsten durchweg unauffälligen Parametern bestimmten wir in SSW 31+0 die Angiogenesemarker: Der sFlt-1/PIGF-Quotient war mit 109,92 erhöht, eine Kontrolle in SSW 32+1 zeigte einen Anstieg auf 183,25. Die Geburt war in einem Haus der Grundversorgung geplant, nun erfolgte die Einweisung in ein Level1-Perinatalzentrum.

In SSW 33+2 wurde dort ein arterieller Hypertonus WHO II mit 100\% der Werte über der Norm diagnostiziert, der sFlt-1/PIGF-Quotient stieg auf 223,01. Es erfolgten eine antihypertensive Therapie und RDS-Prophylaxe.

In SSW 34+1 entwickelte sich innerhalb von 14 Stunden klinisch und laborchemisch ein fulminantes HELLP-Syndrom. Per Sectio wurde ein Mädchen entbunden: $2265 \mathrm{~g}$, APGAR 6/7/9, pHa 7,28.

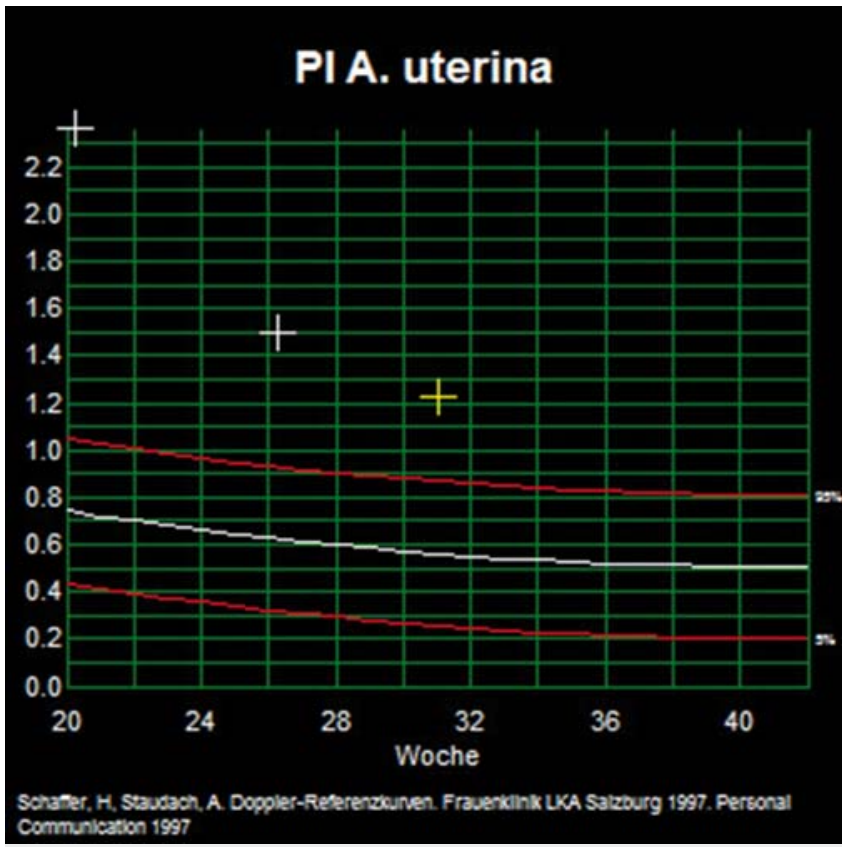

Abb. 1 PI arteria uterina li. 


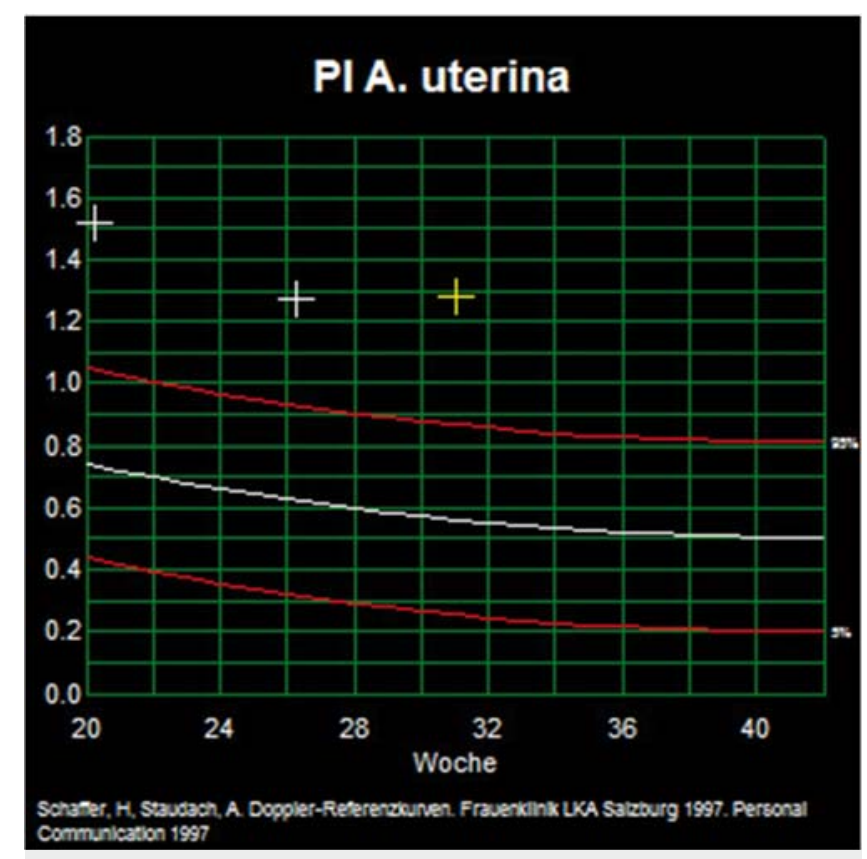

Abb. 2 PI arteria uterina re.

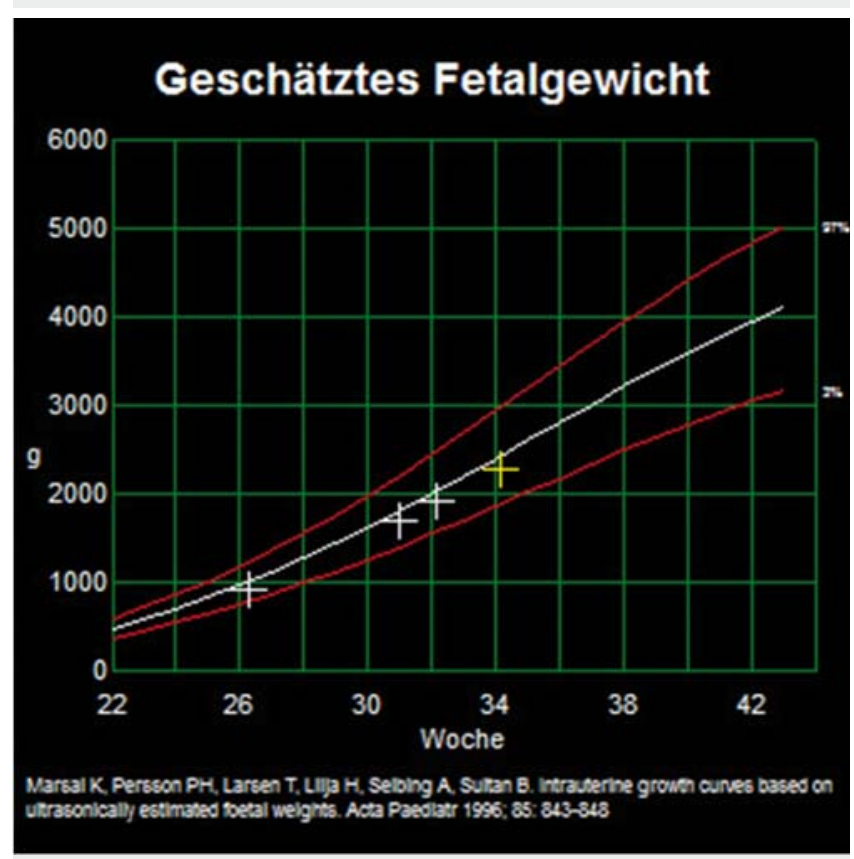

- Abb. 3 Sonografisch geschätztes Fetalgewicht

Schlussfolgerung Ein routinemäßiges Präeklampsiescreening im Rahmen der erweiterten Ersttrimesteruntersuchung erscheint sinnvoll, da die Sensitivität für frühe Präeklampsie mit 93\% hoch und mit ASS 100 ein Medikament zur Prophylaxe verfügbar ist.

Die Bestimmung der Biomarker sFlt-1/PIGF erlaubt ab SSW 20+0 das Erkennen einer angiogenetischen Dysbalance mit einer hohen Sensitivität von 88\%. Dadurch können wir lange vor anders objektivierbaren Parametern ein Präeklampsierisiko erkennen und entsprechende Konsequenzen ziehen.

Der hier beschriebene Fall zeigt somit ein modernes Management der Präeklampsie. Mit hoher Wahrscheinlichkeit konnten die Tragzeit verlängert und das kindliche Outcome verbessert werden.
P3.009 Megazystis-Microcolon intestinales Hypoperistaltik-Syndrom - pränatal eine diagnostische Herausforderung - 2 Fallberichte

Autoren Riße $S^{1}$, Huhle $D^{2}$, Geiler $S^{1}$, Thome $U^{3}$, Faber $R^{1}$ Institut 1 Zentrum für Pränatale Medizin, Leipzig/DE; 2 Praxis für Humangenetik und Kinderheilkunde, Leipzig/DE; 3 Universitätsklinikum Leipzig, Leipzig/DE

DOI 10.1055/s-0037-1607001

Problemstellung Das Megazystis-Microcolon intestinale Hypoperistaltik-Syndrom ist sehr selten. Es ist gekennzeichnet durch eine nicht obstruktiv bedingte Megazystis, ein Microcolon und eine Hypoperistaltik des Darmes. Die Prognose ist infaust. Sonografisches Leitzeichen ist die Megazystis, wie sie sich auch bei anderen Uropathien findet. Differentialdiagnostisch stellt das noch seltenere MMIHS, von dem in 2 Kasuistiken berichtet wird, dagegen pränatal eine Herausforderung dar.

Patienten und Methode Kasuistik I: Bei einer 39-jährigen IIG, IP fällt zur Feindiagnostik ein isoliert hyperechogener Darm auf. Der Karyotyp ist unauffällig weiblich. Eine CF wird ausgeschlossen. Im weiteren Schwangerschaftsverlauf entwickelt sich eine Megazystis und gegen Ende der Schwangerschaft ein Polyhydramnion. Neben einer neurogen gestörten Harnblase wird auch ein MMIHS vermutet. Nach Spontangeburt in der 38. SSW ist eine Spontanmiktion bei dem Neugeborenen nicht möglich. Hinzu kommen massive Darmentleerungsstörungen, an denen das Kind verstirbt. Kasuistik II: Eine 27-jährige IG wird in der 18. SSW mit Verdacht auf ein fetales Syndrom vorgestellt. Es liegen eine Megazystis, ein Double-Bubble-Phänomen und ein Ventrikelseptumdefekt vor. Der Karyotyp ist unauffällig weiblich. Neben einer VACTERL-Assoziation wird wegen der normalen Fruchtwassermenge und gastrointestinalen Auffälligkeiten auch an ein MMIHS gedacht. Aufgrund der hohen Letalität entscheiden sich die Eltern in der 21. SSW zum Schwangerschaftsabbruch.

Ergebnis Bei Kasuistik I bestätigt eine Autopsie das Vorliegen eines MMIHS. Im zweiten Fall erbringt die fetale Array-CGH eine homozygote Mikrodeletion im MYH11-Gen.

Schlussfolgerung Bei der Befundkonstellation Megazystis und normaler Fruchtwassermenge oder Polyhydramnion sollte auch an das seltene MMIHS gedacht werden. Unterdessen ist eine genetische Diagnostik für krankheitsauslösende Mutationen auf zwei Genen möglich.

\section{P3.010 Diagnose einer isolierten Gaumenspalte anhand des „equals sign“ in der Routinediagnostik}

Autoren Bosselmann $S^{1}$, Mielke $G^{1}$

Institut 1 Pränatalzentrum Stuttgart, Stuttgart/DE

DOI 10.1055/s-0037-1607002

Patienten und Methode Eine 36-jährige IG/OP stellt sich zur sonografischen Fehlbildungsdiagnostik mit 22+1 SSW vor. Eigen- und Familienanamnese, sowie der bisherige Schwangerschaftsverlauf sind unauffällig (Screeningsituation). Die Untersuchungsbedingungen sind günstig (BMI 21,4), jedoch gelingt die Darstellung von Rachen und Gaumen lagebedingt nur eingeschränkt. Bei fehlender Darstellbarkeit des „equals sign“ wird der Verdacht auf eine isolierte Gaumenspalte gestellt. Eine sonografische Verlaufskontrolle bei günstigeren Schallbedingungen ergibt den Nachweis einer Spalte des weichen und des harten Gaumens. Die Diagnose wird postnatal bestätigt. 


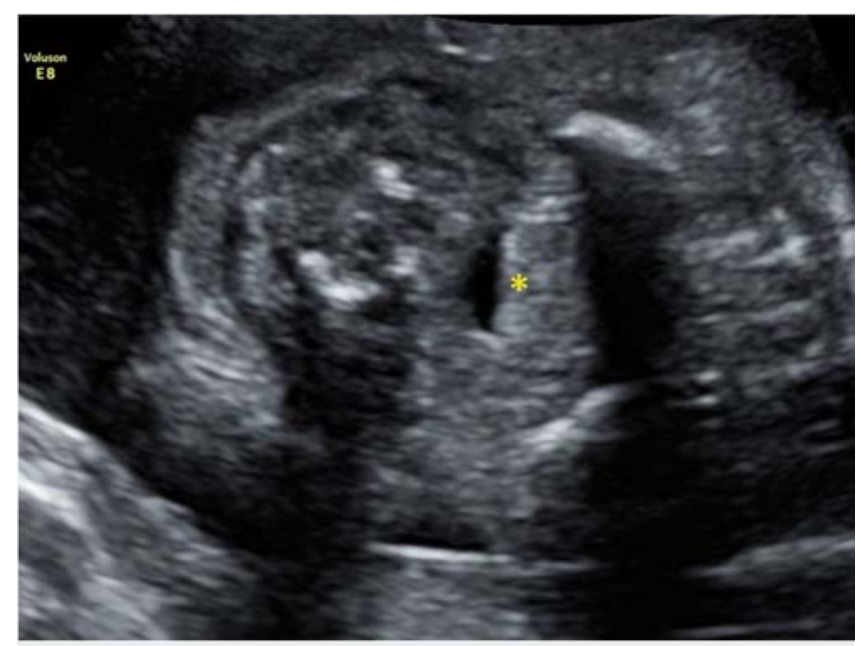

Abb. 1 Fetaler Rachen im Axialschnitt ohne Darstellbarkeit des „equals sign“. Zungengrund $\left({ }^{*}\right)$

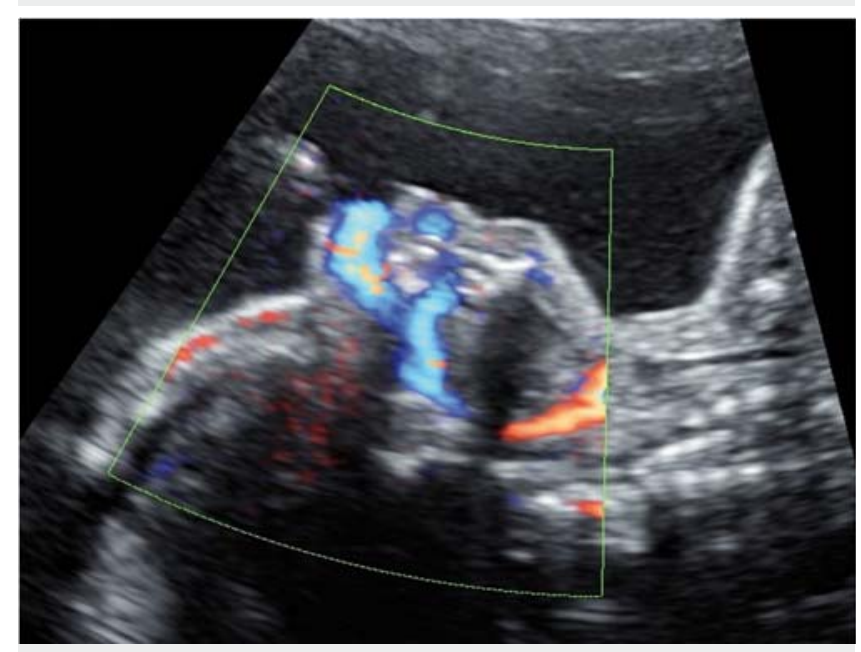

Abb. 2 Dopplersonografische Darstellung der Kommunikation zwischen Mund- und Nasenhöhle im Bereich der Gaumenspalte

Schlussfolgerung Die Diagnose einer isolierten Gaumenspalte wird in der Routinediagnostik relativ selten gestellt. Die klinische Bedeutung einer Gaumenspalte liegt in der Assoziation zu syndromalen Erkrankungen, in der Gefahr von peripartalen Komplikationen (z.B. Verlegung der Atemwege) und nachgeburtlichen Problemen bei der Ernährung, der Sprachentwicklung, sowie der Notwendigkeit von zum Teil mehrfachen Operationen.

Das von Wilhelm und Borgers 2010 beschriebene „equals sign“ zur Beurteilung des weichen Gaumens entspricht im lateralen Axial- oder Coronarschnitt der fetalen Uvula. Im Gegensatz zur direkten Darstellung von weichem Gaumen und Uvula im Sagittalschnitt durch den geöffneten fetalen Mund gelingt die Darstellung des „equals sign“ in der überwältigenden Mehrzahl der Untersuchungen.

Durch das „equals sign“ lässt sich auch in der Screeningsituation die Uvula und damit eine Schlüsselstruktur für die Diagnose von Gaumenspalten mit guter Zuverlässigkeit darstellen. Abzugrenzen ist das „equals sign“ gegenüber den ebenfalls parallelen Linien, die im weiter kaudal gelegeneren Axialschnitt vom oberen Larynx bzw. der Epiglottis gebildet werden.
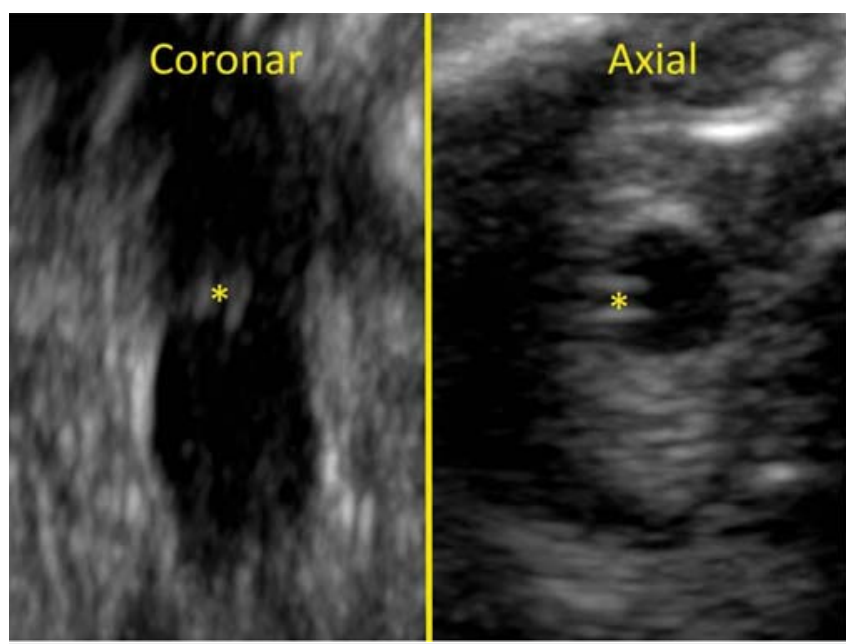

- Abb. 3 Darstellung des „equals sign“ bei unauffälligem weichen Gaumen im Coronar- und Axialschnitt. Uvula/,equals sign“ ( ${ }^{*}$ )

\section{P3.011 Seltene Differentialdiagnose bei schwerer Wachstumsrestriktion: Beckwith-Wiedemann- Syndrom}

Autoren Mommsen $\mathrm{H}^{1}$, Gloning $\mathrm{KP}^{1}$, Shoukier $\mathrm{M}^{1}$, Janke $\mathrm{A}^{1}$, Schramm $\mathrm{T}^{1}$ Institut 1 Pränatal-Medizin München, München/DE

DOI 10.1055/s-0037-1607003

Problemstellung Das Beckwith-Wiedemann-Syndrom fällt pränatal unter anderem durch übermäßiges Wachstum auf. Diese genetisch determinierte Wachstumsregulationsstörung kann selten zu einer auffälligen plazentaren Morphologie und Funktion mit extremer IUWR statt Makrosomie führen. Die pränatale als auch die postpartale Diagnostik kann hierdurch erheblich erschwert werden, da das Leitsymptom Makrosomie fehlt.

Patienten und Methode Erstvorstellung der 22 J. IG in der 19. SSW: Dicke inhomogene Plazenta mit kleiner Haftfläche; Insertio velamentosa; frühe IUWR; kleine Omphalozele mit kleiner Darmschlinge; echodichter Darm. Uterine und fetale Doppler unauffällig. Fruchtwassermenge im oberen Normbereich. Plazentabiopsie: unauffälliges Karyogramm. MolGen: CF negativ. ArrayCGH abgelehnt. Stationäre Überwachung ab der 25. Woche: abflachendes Wachstum, zunehmende klinische und laborchemische Präeklampsiezeichen, weiterhin unauffälliger Doppler. Entbindung in der 28+0. Woche bei schwerer progredienter Präeklampsie durch primäre Sectio.

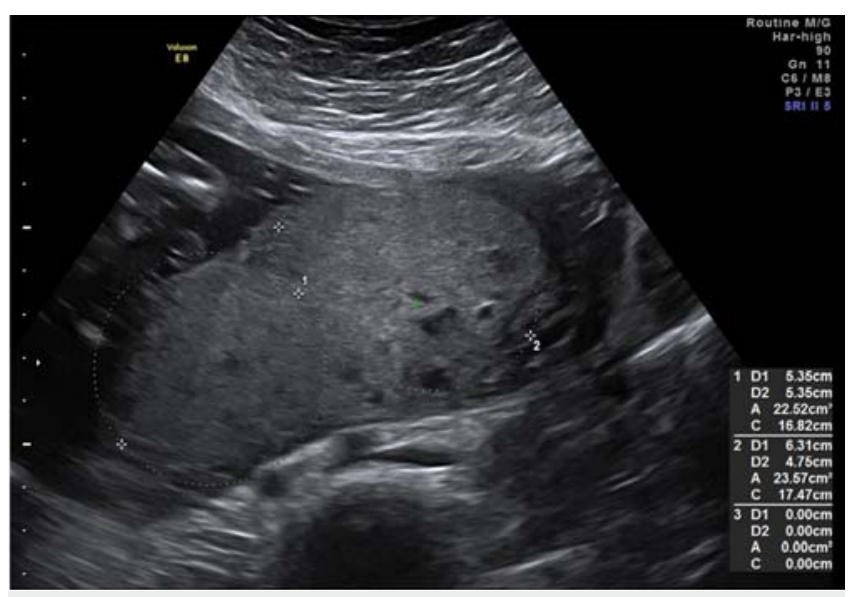

Abb. 1 Schwere IUWR: Plazenta 
Ergebnis Weiblich, $860 \mathrm{~g}$, Apgar 4/7/9, pH 7,17. Neonatologische Behandlung mit teils HFO-Beatmung bis zum 16. Tag. Glukoseregulationsstörung, Hypothyreose. Sonst weitgehend komplikationsfreier Verlauf. Dysmorphologie: IUWR <3. Perz., kleine nicht OP-bedürftige Omphalozele, Makroglossie. Genetik: Array-CGH unauffällig. Molekulargenetik: Hypomethylierung von KCNQ 1OT1:TSS-DMR (ICR2): Beckwith-Wiedemann-Syndrom. Plazentahistologie: Hyperplastische Plazenta $(600 \mathrm{~g})$, Insertio velamentosa. Ausgedehnte Gitterinfarkte, intervillöse Fibrinablagerungen und Zottenstromafibrose.

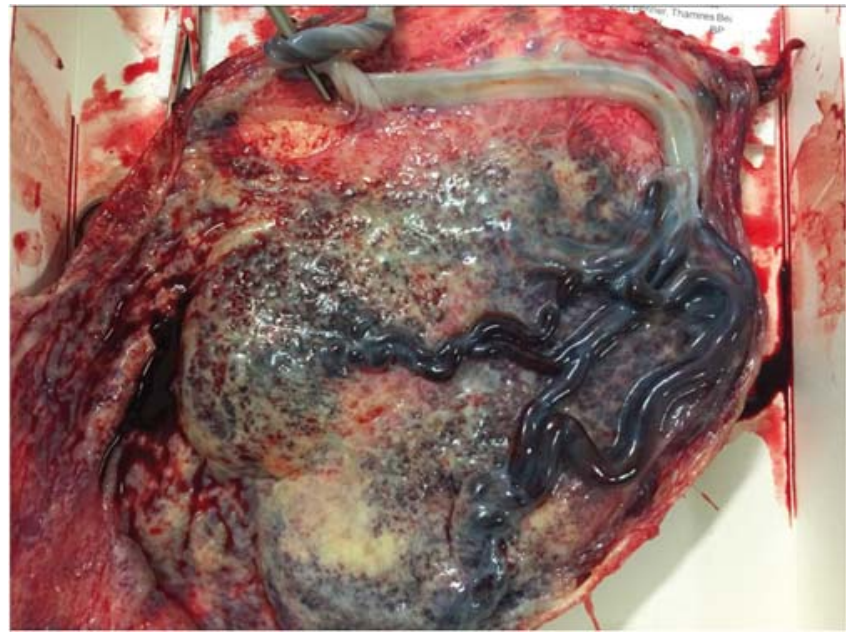

Abb. 2 Schwere IUWR und BWS: Plazenta

Schlussfolgerung Insbesondere bei atypischer IUWR mit unauffälliger Fruchtwassermenge und Dopplerparametern liegt häufig ein genetisches Syndrom vor. Die möglichst genaue pränatale sonografische Fehlbildungsdiagnostik ist wichtig, um eine zielgerichtete genetische Klärung zu veranlassen. Bei unklaren Fällen ist die postpartale dysmorphologisch-syndromologische Diagnostik unerlässlich, um die korrekte Diagnose stellen zu können.

Ein BWS mit plazentar bedingter Wachstumsrestriktion ist extrem ungewöhnlich und wurde als plazentare mesenchymale Dysplasie beschrieben. Bei untypischer IUWR mit kleiner Omphalozele und Makroglossie sollte auch daran gedacht werden.

Die molekulargenetische Heterogenität der IUWR wird hierbei deutlich.

\section{P3.012 Qualität der (semi-)automatischen,} sonografischen Beurteilung des fetalen Herzens (Fetal Intelligent Navigation Echocardiography „FINE“): eine Frage der „Zeit"?

Autoren $\underline{\text { Hartge } \mathrm{D}^{1}}$, Fernandes $\mathrm{T}^{1}$, Gembicki $\mathrm{M}^{1}$, Dracopoulos $\mathrm{C}^{1}$, Weichert $]^{1}$ Institut 1 Universitätsklinikum Schleswig-Holstein, Lübeck/DE

DOI 10.1055/s-0037-1607004

Problemstellung Beurteilung der Möglichkeiten und Grenzen des sinnvollen Einsatzes von „FINE“ bei idealen und ungünstigen fetalen Lagen.

Patienten und Methode Prospektive Analyse der korrekten Darstellung der 9 Standardebenen zur fetalen Echokardiografie in 653 STIC-Volumina evaluiert mit der „FINE“ software mit einer fetalen Rückenlokalisation bei 5- 7 Uhr, 2 und 10 Uhr, 3 und 9 Uhr und 4 und 8 Uhr während der initialen Volumenakquise.

Ergebnis Von 04/2015-04/2017 wurden 653 „FINE“ Volumina gewonnen. Das mittlere Gestationsalter lag bei $20^{5 / 7} \mathrm{SSW}\left(14^{0 / 7}-32^{0 / 7} \mathrm{SSW}\right)$.

Dabei lag der fetale Rücken während der initialen Volumenakquise in 340 Fällen zwischen $5-7$ Uhr, in 224 bei $4 \& 8$ Uhr, in 69 bei $3 \& 9$ Uhr und in 20 Fällen bei $2 \& 10$ Uhr.
Die Visualisierung von $\geq 8$ Ebenen gelang in $81 \%$ der Fälle mit dem Rücken zwischen 5 - 7 Uhr, in $74 \%$ bei 4 \& 8 Uhr, in $49 \%$ bei 3 \& 9 Uhr und in $70 \%$ der Fälle mit dem fetalen Rücken bei 2 \& 10 Uhr.

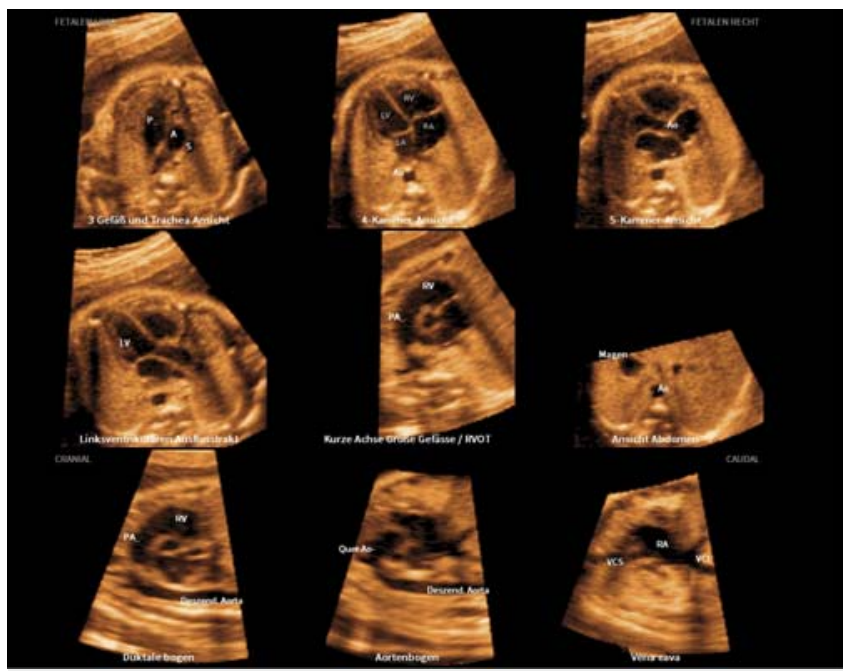

D Abb. 19 Standardebenen bei „FINE“

Schlussfolgerung Der Einsatz von „FINE“ ist selbst dann sinnvoll möglich, wenn sich der Fet nicht in einer idealen Position befindet.

Die bedeutendsten Störfaktoren für eine gelungene Anwendung von „FINE“ sind vor allem Schallauslöschungen durch knöcherne Strukturen, sowie eine erschwerte Beurteilbarkeit von Herzstrukturen, die sich parallel zur ursprünglichen Insonation präsentierten.

\section{P3.013 Performance und inter-rater Reliabilität der FINE (Fetal Intelligent Navigation Echocardiography, 5D Heart) Technik bei angeborenen Herzfehlern}

Autoren Weichert $A^{1}$, Tucher E von ${ }^{1}$, Weichert $J^{2}$, Kalache $K^{3}$, Henrich $W^{1}$ Institut 1 Charité - Universitätsmedizin Berlin, Berlin/DE;

2 Universitätsklinikum Schleswig-Holstein, Lübeck/DE; 3 Sidra Medical and

Research Center, Doha/QA

DOI 10.1055/s-0037-1607005

Problemstellung Angeborene Herzfehler gehören zu den häufigsten organspezifischen Fehlbildungen und sind die Hauptursache neonataler Morbidität und Mortalität. In Abhängigkeit von dem untersuchten Studienkollektiv liegt ihre Inzidenz zwischen 4/1000 and 50/1000 Lebendgeburten.

Trotz nationaler und internationaler Leitlinien mit standardisierten Untersuchungsebenen und weitreichender Verfügbarkeit von Ultraschall wird die Mehrheit der betroffenen Fälle erst nach der Geburt diagnostiziert. Die FINE (Fetal intelligent navigation echocardiography, 5D Heart) Technik ermöglicht dem Untersucher die leitlinienbasierten Untersuchungsebenen aus einem STIC Volumen zu extrahieren.

Patienten und Methode In unserer retrospektiven Beobachtungsstudie wurden STIC Volumina von Feten mit angeborenen Herzfehlern und Anomalien identifiziert $(n=60)$. Die Herzfehler schließen HLHS, AVSD, DORV und andere größere Fehlbildungen des Herzens ein.

Nach Markierung von sechs festgelegten Wegmarken wurden neun Untersuchungsebenen extrahiert und dargestellt. Die Ebenen wurden auf Darstellbarkeit der Pathologie geprüft und weiteren verblindeten Untersuchern zur Beurteilung vorgelegt.

Ergebnis Wir konnten zeigen, dass verblindete Untersucher den Herzfehler anhand des Volumens in einem hohen prozentualen Anteil identifizieren können. Dies könnte Untersucher in Regionen ohne direkten Zugang zu einem auf angeborene Herzfehler spezialisierten Zentrum unterstützen, die pränatale Betreuung Schwangerer zu verbessern. 
Schlussfolgerung Unsere Studie bestätigt bereits publizierte Daten, dass die FINE/5D Heart Technik ein nützliches Werkzeug ist, cardiale STIC Volumina zu untersuchen und angeborene Herzfehler zu beurteilen.

\section{P3.014 Die Bedeutung des isolierten, kurzen Femurs < 5. Perzentile für das kindliche Outcome und die Gewichtsschätzung ante partum}

\author{
Autoren Beyer $]^{1}$, Lauten $A^{1}$, Jahn $C^{1}$, Schlembach $D^{2}$, Schleußner $E^{1}$, \\ Schneider $\mathrm{U}^{1}$ \\ Institut 1 Universitätsklinikum Jena, Jena/DE; 2 Vivantes Klinikum Neukölln, \\ Berlin/DE
}

DOI 10.1055/s-0037-1607006

Problemstellung Die fetale Femurlänge zählt zu den 3 etablierten Grundparametern für die fetale Gewichtsschätzung. Eine isolierte Femurlänge $<5$. Perzentile kann im zweiten Trimenon ein frühes Hinweiszeichen für eine IUGR oder einen SGA-Feten sein. Bei Feten mit einer Femurlänge < 5. Perzentile wird vermutet, dass die gängigen Formeln zur Gewichtsschätzung systematisch zu leicht schätzen.

Patienten und Methode Es wurden retrospektiv Daten von 2231 Patientinnen ausgewertet, die im Zeitraum von 2010 - 2012 am Universitätsklinikum Jena entbunden wurden. Ausgeschlossen wurden Mehrlinge und Totgeburten. Die biometrischen Daten der Feten wurden jeweils mit verschiedenen etablierten Formeln berechnet. Die statistische Auswertung erfolgte mit dem Bland-Altman Plot und Bestimmtheitsmaß.

Ergebnis Von 2231 Patientinnen hatten 80 Feten mit einem Femur < 5. Perzentile. 33 davon waren SGA-Kinder < 10. Perzentile (41,3\%), 17 (52\%) der SGA-Kinder waren Frühgeborene. Beides ist signifikant höher als im Gesamtkollektiv. Der Vergleich der Formel zur Gewichtsschätzung zeigt, dass Hadlock III und Ott das fetale Gewicht am genauesten schätzen. Dies galt sowohl im Gesamtkollektiv, als auch bei SGA-/IUGR-Kindern. Zu den „ungenauesten“ Formeln zählten die von Schillinger und Hansmann. Die Vermutung, dass die gängigen Formeln das Gewicht bei Feten mit kurzem Femur systematisch unterschätzen bestätigt sich nicht.

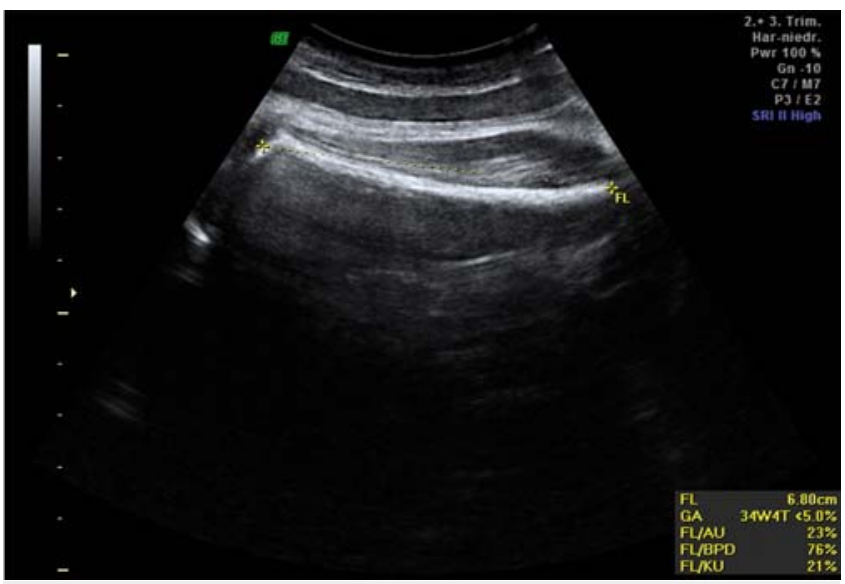

Abb. 1 Femur <5. Perzentile

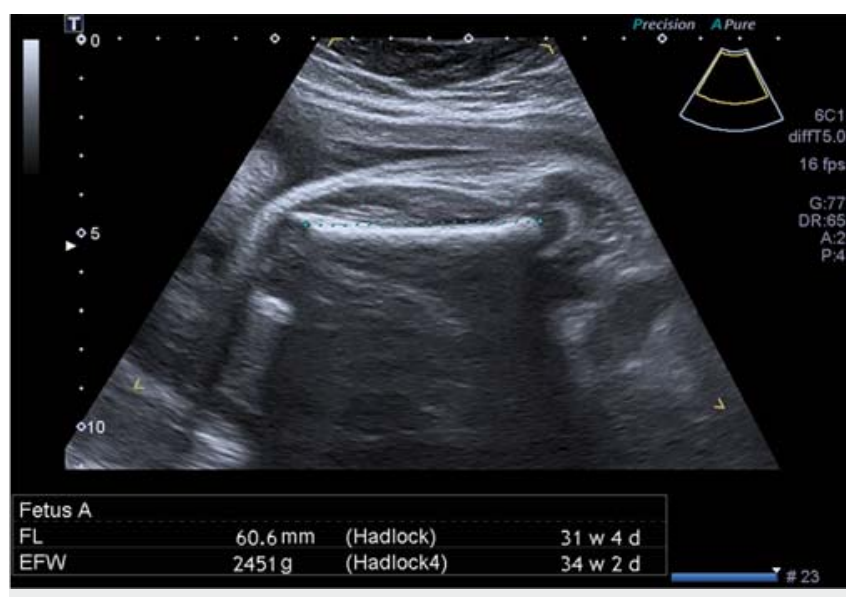

Abb. 2 2. Beispiel

Schlussfolgerung Wir können bestätigen, dass eine Femurlänge $<5 \%$ bei Feten ist mit einer signifikant höheren Rate an Frühgeburten und SGA-Kindern assoziiert ist. Diese Schwangerschaften sollten engmaschig kontrolliert werden. Die Gewichtsschätzung mit den üblichen Formeln (besonders nach Hadlock III) zeigt keine relevanten Abweichungen, diese können demzufolge auch in diesem Kollektiv mit den bekannten Grenzen genutzt werden.

\section{P3.015 Einfluss der Bildrate auf die Strainanalyse bei zweidimensionalem Wall Motion Tracking in der fetalen Echokardiografie}

Autoren Enzensberger $C^{1}$, Rostock $L^{1}$, Götte $\mathrm{M}^{1}$, Wolter $A^{1}$, Herrmann $]^{2}$, Axt-Fliedner $\mathrm{R}^{1}$

Institut 1 Zentrum für Frauenheilkunde und Geburtshilfe,

Universitätsklinikum Gießen \& Marburg, Gießen/DE; 2 Statistikberatung

Gießen, Gießen/DE

DOI 10.1055/s-0037-1607007

Problemstellung Die beim Speckle Tracking in der fetalen Echokardiografie angewandten Bildraten zur Strainanalyse sind in der Literatur variabel angegeben. Durch die erhöhte fetale Herzfrequenz erscheint eine hohe Bildrate notwendig. Diese Studie untersuchte den Effekt der Bildrate auf die Strainanalyse beim 2D Speckle Tracking.

Patienten und Methode In einer prospektiven Studie an Patientinnen der pränataldiagnostischen Ambulanz wurde ein Toshiba Aplio 500 zur fetalen Echokardiografie verwendet. Es wurden Videosequenzen eines fetalen Herzzyklus im apikalen oder basalen Vierkammerblick erstellt und in zwei Bildformaten gespeichert, mit der originalen Bildrate (acoustic frame rate, AFR) sowie im DICOM-Format (Digital Imaging and Communications in Medicine) mit 60 Bildern pro Sekunde (Bilder/s =fps, $60 \mathrm{fps}$ ). Unter Verwendung von Wall Motion Tracking wurden globale longitudinale maximale Strain-Werte für den linken (LV) und rechten (RV) Ventrikel ermittelt.

Ergebnis Es wurden $n=103$ gesunde Feten zwischen der 17. - 39. SSW eingeschlossen. Die originale Bildwiederholungsrate lag bei $127 \pm 26 \mathrm{fps}$. Zwischen DICOM- und AFR-Auswertung zeigte sich bezüglich des globalen longitudinalen maximalen Strains für beide Ventrikel ein hoch signifikanter Mittelwertunterschied ( $L V=2,89$; $R V=2,51$; beide $p<0,001$ ), wobei die DICOMWerte im Mittel unter den AFR-Werten lagen.

Nach Bland-Altmann-Analyse kann erwartet werden, dass 95\% der Messungen DICOM-Werte im Bereich zwischen 1,36 (LV) bzw. 1,13 (RV) höher als der jeweilige AFR-Wert und bis 7,13 (LV) bzw. 6,15 (RV) niedriger als der AFRWert ergeben.

Schlussfolgerung Die Bildrate hat einen signifikanten Einfluss auf die Messwerte. Die Vergleichbarkeit von mit unterschiedlichen Bildraten erhobenen Strain-Werten ist hierdurch etwas eingeschränkt. Eine Notwendigkeit für hohe Bildraten (>100 fps) zur Strain-Analyse ergibt sich hieraus jedoch nicht. 


\section{P3.016 Adipositas bei Risikoschwangeren}

Autoren Eggensberger $\mathrm{T}^{1}$

Institut 1 Caritaskrankenhaus St. Josef, Regensburg/DE

DOI 10.1055/s-0037-1607008

Problemstellung Bei Adipositas wird eine höhere Rate an fetalen Anomalien beschrieben. Andererseits ist die Sensitivität für die Entdeckung von fetalen Anomalien in der pränatalen Ultraschalldiagnostik bei Adipösen geringer.

Nimmt der Anteil adipöser Patientinnen im Risikokollektiv für fetale Erkrankungen zu?

Patienten und Methode Im Zentrum für Pränatalmedizin am Caritas Krankenhaus St. Josef in Regensburg wurden Schwangere in der 20. bis 22. SSW von 2004 bis 2016 in die Studie eingeschlossen. Untersucht wurde der Anteil an Übergewichtigen mit einem BMI $\geq 25$. Der BMI wurde aus der im Mutterpass dokumentierten Größe sowie dem aktuellen Gewicht der Patientin errechnet. Zudem analysierten wir das Ausmaß der Adipositas.

Ergebnis Im Beobachtungszeitraum wiesen 53,5\% 1,19 [52,1\% - 54,7\%] der Patientinnen zum Untersuchungszeitpunkt einen BMI $\geq 25$ auf.

\begin{tabular}{|l|l|}
\hline Jahre & BMI $\geq 25$ (in\%) \\
\hline $2004 / 2005$ & 52,1 \\
\hline $2006 / 2007$ & 54,7 \\
\hline $2008 / 2009$ & 52 \\
\hline $2010 / 2011$ & 54,7 \\
\hline $2012 / 2013$ & 52,7 \\
\hline $2014 / 2015$ & 54,2 \\
\hline 2016 & 54 \\
\hline
\end{tabular}

Betrachtet man den Schweregrad der Adipositas lässt sich ein steigender Anteil der Patientinnen mit höhergradiger Adipositas erkennen. Insgesamt weisen 20,4\% der Patientinnen einen BMI $\geq 30,7,8 \%$ einen $\mathrm{BMI} \geq 35$ und $2,9 \%$ einen $\mathrm{BMI} \geq 40$ auf. Der Anteil der Patientinnen mit einem $\mathrm{BMI} \geq 40$ stieg von 2,1\% zwischen den Jahren 2004 bis 2005 auf einen Anteil von 3,7\% im Jahr 2016 an.

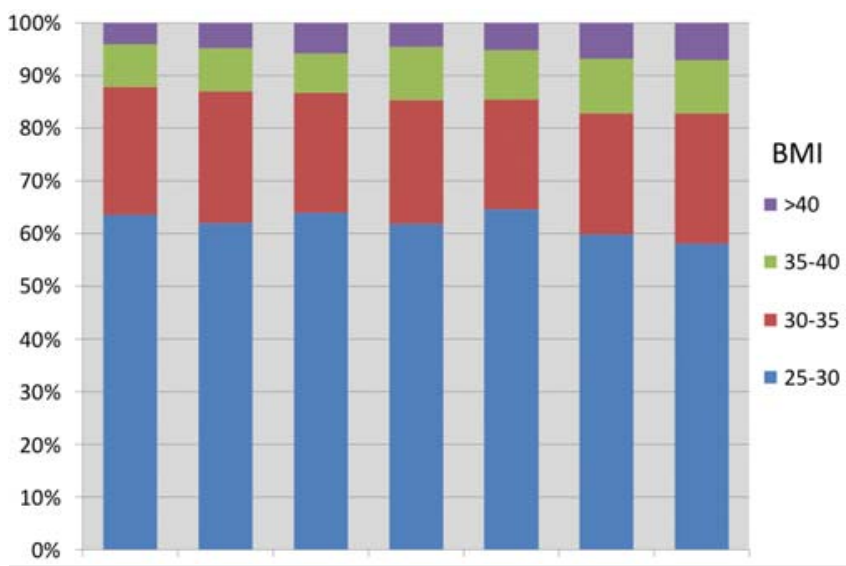

Abb. 1 Verteilung der Adipositasgrade in der 20. - 22. SSW

Schlussfolgerung Der Anteil adipöser Patientinnen war im Beobachtungszeitraum von 2004 bis 2016 mit einem Anteil von über 52\% stabil. Es zeigte sich jedoch eine kontinuierliche Zunahme der Patientinnen mit höhergradiger Adipositas.

\section{P3.017 Asymmetrie der Plexus choroidei im ersten Trimenon als mögliches frühes Zeichen einer fetalen Hemimegalencephalie (HME)}

Autoren $\underline{\text { Weichert } A^{1}}$, Becker $\mathrm{R}^{2}$, Kalache $\mathrm{K}^{3}$, Tucher E von ${ }^{1}$, Henrich $\mathrm{W}^{1}$, Entezami $\mathrm{M}^{2}$

Institut 1 Charité - Universitätsmedizin Berlin, Berlin/DE; 2 Zentrum für Pränataldiagnostik - Kurfürstendamm 199, Berlin/DE; 3 Sidra Medical and

Research Center, Doha/QA

DOI 10.1055/s-0037-1607009

Patienten und Methode Die Darstellung der fetalen axialen BPD Ebene beim Ersttrimesterscreening (ETS) schließt die Plexus choroidei (PC) mit dem sogenannten „Butterfly sign“ ein, welches als eines der sensitivsten Zeichen für strukturelle Hirnfehlbildungen im ersten Trimenon gilt.

Während Formveränderungen mit Fusion der PC mit einer Holoprosencephalie assoziiert sind, werden Missverhältnisse der PC und zum Ventrikeldurchmesser/der Ventrikelfläche als frühe Zeichen einer Ventrikulomegalie gedeutet. PC-Assymmetrien gelten als frühe Zeichen der physiologischen Hirnlateralität. Die Informationen hinsichtlich der klinische Relevanz sind jedoch begrenzt. HME ist eine extrem seltene Hirnfehlbildung, die durch eine vergrößerte Hemisphäre mit und ohne Zeichen corticaler Entwicklungsstörungen characterisiert ist.

Wir berichten von einem Fall einer 25-jährigen Patientin (G1, P0), die initial zum ETS in $13+3$ SSW überwiesen wurde. Bis auf eine milder PC Differenz war der Befund unauffällig und resultierte in einem adjustierte Risiko für eine Trisomie 21 von 1:4134 und 1:7622 für eine Trisomie 13/18.

In $22+2$ Schwangerschaftswochen zeigte der Fetus eine Makrozephalie mit abnormalien Wachstum mit corticaler Entwicklungsstörung der rechten Hemisphäre mit einseitiger Ventrikulomegalie, die zu einer Mittellinienverschiebung und Kompression der linken Hemisphäre führte. Die Verdachtsdiagnose lautete HME, die mittels 2D und 3D Ultraschall sowie MRT bestätigt werden konnte.

Schlussfolgerung Zusätzlich zu vorangegangenen Studien belegt dieser Fall, dass eine PC Asymmetrie auch ein frühes Zeichen einer abnormalen Laterilität wie bei einer HME sein könnte. Wir demonstrieren die diagnostischen Befunde und das pränatale Management.

\section{P3.018 Fetales Bardet-Biedl-Syndrom mit polyzystischer Nierendysplasie bds., Polydaktylie, Dolichozephalie und vergrößerter Cisterna magna bei Konsanguinität}

Autoren Beyer J $]^{1}$, Lauten $A^{1}$, Fröber $R^{1}$, Schleußner $E^{1}$, Schneider $U^{1}$ Institut 1 Universitätsklinikum Jena, Jena/DE

DOI 10.1055/s-0037-1607010

Patienten und Methode Bardet-Biedl-Syndrom (BBS). Das BBS ist eine rare (Inzidenz ca. 1:160.000) komplexe syndromale Erkrankung, eine Ziliopathie. Die Symptome sind heterogen, typisch sind Adipositas, Hypogenitalismus, mentale Retardierung (ca. 50\%, moderat) und postaxiale Polydaktylien (inkonstant) sowie in nahezu immer eine Pigmentretinopathie und in ca. $62 \%$ zystische Nierendysplasien mit progredienter Insuffizienz. Die Zeichen sind häufig erst nachgeburtlich zu erkennen, eine antenatale Diagnose ist äußerst selten. Ätiologisch liegt eine autosomal rezessive Vererbung zu Grunde, 19 Genloci konnten bislang identifiziert werden (BBS-Komplex). Die Prognose wird durch den Grad der geistigen Retardierung sowie der Progredienz der Niereninsuffizienz und der Einschränkung des Sehvermögens bestimmt.

Kasuistik 20-jährige I. Gravida, 0 Para, Erstvorstellung zur Feindiagnostik bei 18/5 und 20/6 SSW. in unserer Ambulanz bei Verwandtenehe. Fruchtwasser im unteren Normbereich, Dolichocephalie, vergrößerte Cisterna magna, vergrößerte, polyzystische Nieren bds., Hexadaktylie. Dringender V.a. komplexe syndromale Erkrankung mit autosomal rezessivem Vererbungsmuster. Amniocentese (45XX T15/21), humangenetische Beratung erfolgt. Nach perinatologischem Konsil ist erfolgt die Schwangerschaftsbeendigung bei $21 / 6$ 
SSW. Weiblicher Fet, $480 \mathrm{~g}, 27 \mathrm{~cm}$. Im paidopathologischen Befund können die sonografisch detektierten Veränderungen post mortem dargestellt und bestätigt werden. Molekulargenetisch Diagnose eines BBS.

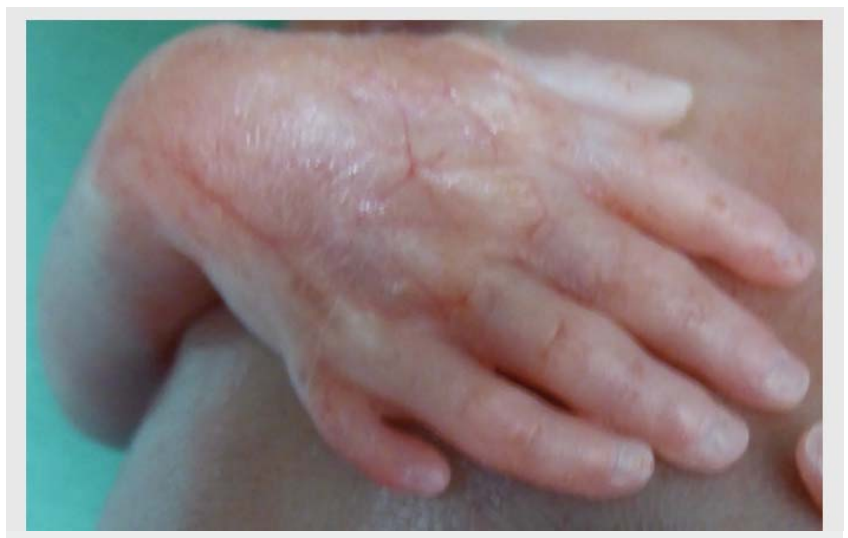

Abb. 1 postaxiale Polydaktylie

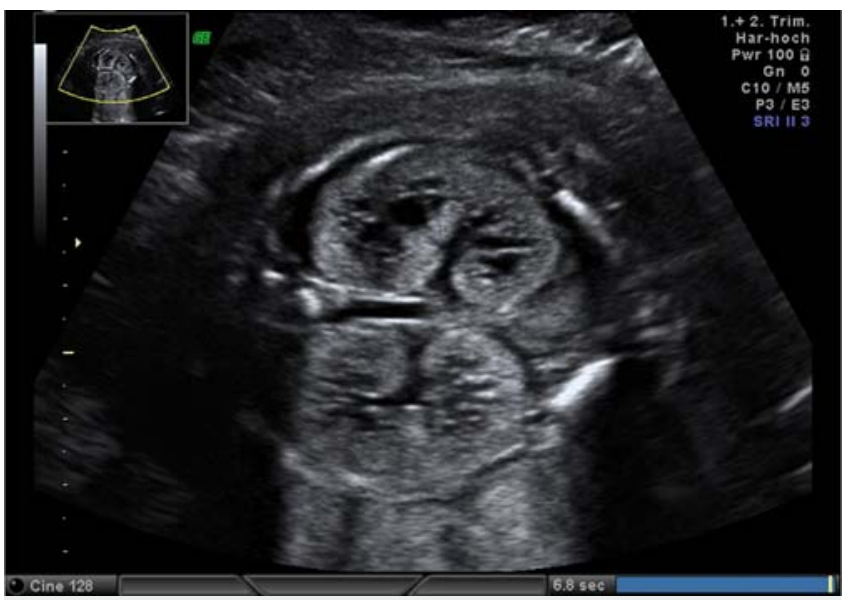

Abb. 2 polyzystische, vergrößerte Nieren bds.

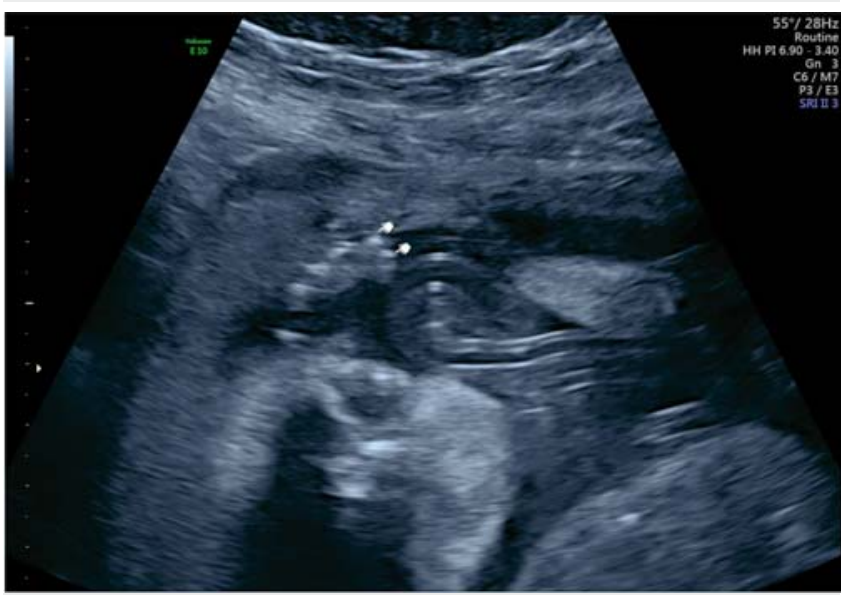

Abb. 3

Schlussfolgerung Aufgrund der komplexen Veränderungen kann von einer schlechten Prognose ausgegangen werden. Die antenatale Diagnose eines Bardet-Biedl-Syndroms ist extrem selten, da die meisten Symptome erst postnatal auffallen. Wegweisend sind a.e. die Nierenveränderungen sowie die Polydaktylie. Auf das $25 \%$ ige Wiederholungsrisiko für Folgeschwangerschaften muss das Paar hingewiesen werden. Die Beratungssituation bleibt auch bei antenataler Diagnose schwierig aufgrund der Heterogenität und der inkons- tanten Ausbildung einer geistigen Retardierung und sollte die Humangenetiker einbeziehen.

\section{P3.019 Inter- und intraobserver-Variabilität in der Beurteilung des fetalen Herzens unter Nutzung der 5DHeart ${ }^{\mathrm{TM}}$-Technologie}

Autoren Gembicki $\mathrm{M}^{1}$, Hartge $\mathrm{D}^{1}$, Weichert ${ }^{1}$

Institut 1 Universitätsklinikum Schleswig-Holstein, Lübeck/DE

DOI 10.1055/s-0037-1607011

Problemstellung Es wurde die inter- und intraobserver-Variabilität und die hiermit korrespondierende Lernkurve in der Beurteilung des fetalen Herzens unter Nutzung einer semi-automatisierten Software (5DHeart ${ }^{\mathrm{TM}}$ ) untersucht. Patienten und Methode Insgesamt 30 gespeicherte Volumendatensätze von Feten aus dem zweiten Trimester wurden von drei Ärzten mit unterschiedlicher Expertise in fetaler Echokardiografie (Beginner, Fortgeschrittener, Experte) mittels der 5DHeart ${ }^{\mathrm{TM}}$-Software zu zwei unterschiedlichen Zeitpunkten (t0, t1) im Abstand von 4 Wochen untersucht.

Die Daten wurden hinsichtlich Untersuchungszeit und korrekter Darstellung der diagnostischen Ebenen des fetalen Herzens von einem verblindeten Expertenpanel analysiert.

Ergebnis Alle Untersucher konnten die Analyse an sämtlichen Datensätzen durchführen. Zu Zeitpunkt t0 war die interobserver-Variabilität sowohl zwischen dem Beginner und dem Fortgeschrittenen $(P=0,0013)$ als auch dem Experten $(P<0,0001)$ hoch.

Bezüglich der intraobserver-Variabilität zeigte der Beginner eine deutliche Verbesserung von t0 zu t1 ( $P=0,0087)$ wohingegen der Fortgeschrittene und der Experte keine signifikante Veränderung zeigten $(P=1,000 / P=0,8383)$.

Der Beginner wies bezüglich der mittleren Untersuchungszeit eine bemerkbare Verbesserung auf (t0: 82,8 s; t1: 73,4 s; P=0,0895). Nichtsdestoweniger waren sowohl der Fortgeschrittene als auch der Experte schneller (t1: 20,9 s/ $28,3$ s, jeweiliges $P<0,0001)$.

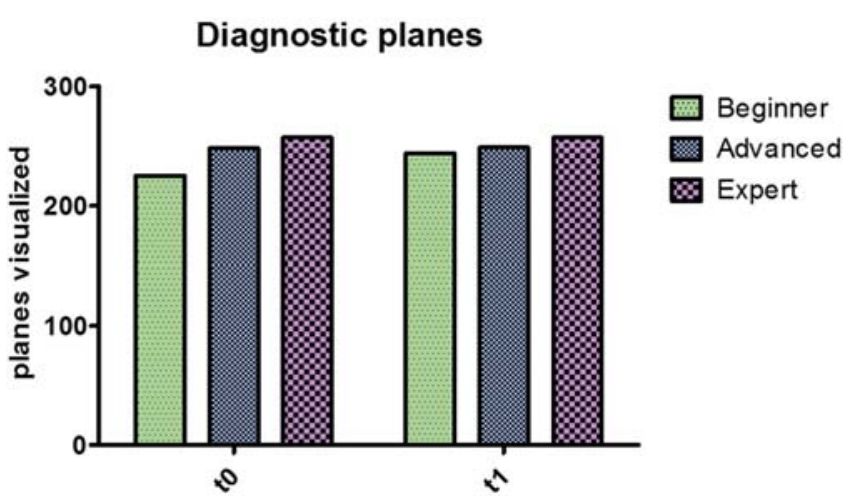

\section{Examination time}

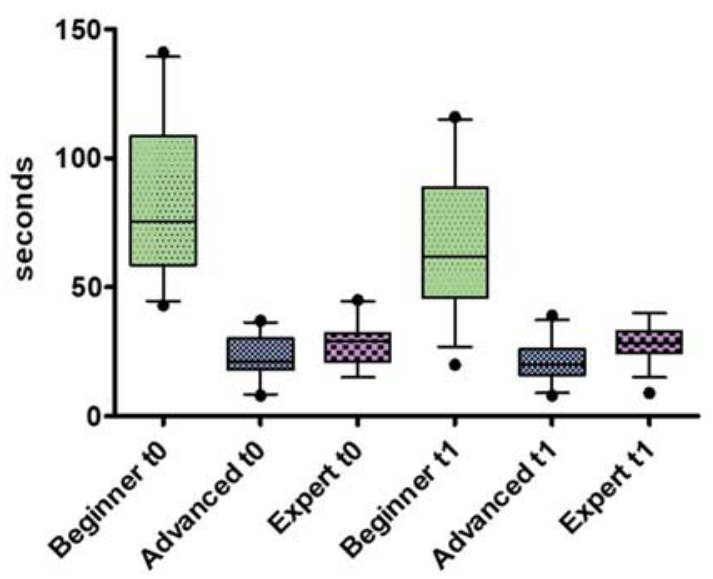


Schlussfolgerung Anhand unserer Daten konnte gezeigt werden, dass die 5DHeart ${ }^{\mathrm{TM}}$-Technologie in der praktischen Anwendung eine zuverlässige und leicht zu erlernende Methode ist. Der Einsatz einer (semi-)automatisierten Software kann die Beurteilung des fetalen Herzens auf eine standardisierte und zeitsparende Art und Weise unterstützen. Software-basierte Ansätze zur fetalen Echokardiografie könnten die Detektion kardialer Anomalien - besonders in ungeübten Händen - verbessern.

\section{P3.020 Fertilität, Schwangerschaft, Post-Partum und Schilddrüse}

Autoren Fernandez Sanchez J ${ }^{1}$

Institut 1 Robert-Bosch-Krankenhaus Stuttgart, Stuttgart/DE

DOI 10.1055/s-0037-1607012

Problemstellung Evaluation einer Funktionsstörung der Schilddrüse und deren Diagnostik bei Kinderwunsch, im Rahmen der Schwangerschaft, sowie Post-Partum.

Patienten und Methode Bei Patientinnen mit einer Schilddrüsenerkrankung werden anhand eigener Erfahrung $(n=12)$ mit Revision der Literatur die unterschiedlichen Szenarien (Hypothyreose, Hyperthyreose) mit Wirkung auf die Fertilität und Verlauf der Schwangerschaft, sowie der Post-Partum-Periode und die diagnostischen Maßnahmen analysiert.

Ergebnis Die häufigste Ursache einer Hypothyreose bei Kinderwunsch und während der Schwangerschaft ist die Hashimoto Thyreoiditis (HT). Hier sollte das mögliche sonografische Spektrum der HT ausreichend bekannt sein (diffuse und fokale Formen), um falsche Diagnosen zu vermeiden, insbesondere bei der fokalen nodulären Form. Bei Patientinnen mit unerfülltem Kinderwunsch und TSH supprimiert bzw. erniedrigt, sollte eine weitere Abklärung mittels Sonografie und evtl. auch mit Szintigrafie erfolgen. Die häufigste Ursache einer Schilddrüsenüberfunktion in der Schwangerschaft ist die B-HCG induzierte Hyperthyreose. Neben der Labordiagnostik ist eine sonografische Untersuchung (B-Bild, Color Doppler, Elastosonografie) notwendig, um eine korrekte Differenzierung zwischen B-HCG und M. Basedow durchführen zu können. Auch ausreichende sonografische Kenntnisse für die Diagnose einer postpartalen Thyreoiditis mit korrekter Evaluation der möglichen Phasen der Erkrankung sind erforderlich.

Schlussfolgerung Eine systematische, laborbiochemische und bildgebende Diagnostik einer Funktionsstörung der Schilddrüse bei unerfülltem Kinderwünsch und während der Schwangerschaft sowie in der Post-Partum-Periode ist hinsichtlich der therapeutischen Konsequenzen notwendig, um Komplikationen zu vermeiden. Diese Diagnostik wird präsentiert und diskutiert.

\section{P3.021 Freie Trisomie 9 im 2. Trimenon}

Autoren Kreiselmaier $\mathrm{P}^{1}$, Becker $\mathrm{WH}^{2}$, Krapp $\mathrm{M}^{1}$

Institut 1 amedes experts Hamburg, Hamburg/DE; 2 Perinatalzentrum

Altona, Hamburg/DE

DOI 10.1055/s-0037-1607013

Problemstellung Die freie Trisomie 9 ist eine seltene Chromosomenstörung, die in den meisten Fällen in einem Frühabort im ersten Trimenon endet, während nur wenige Feten bis zum Erreichen des zweiten Trimenons überleben. Häufiger finden sich Feten mit einer Mosaik Trisomie 9. Das Krankheitsbild ist sehr heterogen. Wir berichten über einen Feten mit freier Trisomie 9 mit multiplen Fehlbildungen.

Patienten und Methode Die 32-jährige GVI/PI kam zur Fehlbildungssonografie in unsere Sprechstunde. Es wurde eine transabdominale Untersuchung in der 21+2 SSW (Philips iU 22) durchgeführt.

Ergebnis Es zeigte sich eine symmetrische Wachstumsrestriktion. Bei gesichertem Gestationsalter lagen alle entsprechenden biometrischen Parameter an bzw. knapp unterhalb der 5. Perzentile. Ferner zeigte sich ein „strawberry sign“ und ein symmetrischer Hydrozephalus. Das Mittelgesicht war hypoplastisch und im Seitenvergleich zeigte sich eine Mikrophtalmie des linken Auges. In der farbkodierten Echokardiografie wurde eine valvulären Aorten- stenose diagnostiziert. Zusätzlich fanden sich ein singulärer hyperechogener Focus links, eine singuläre Nabelschnurarterie rechts und „clenched fingers“. Aufgrund dieser Befunde wurde eine Amniozentese durchgeführt. Der Schnelltest für die häufigen Chromosomenstörungen (Trisomie 21,18 und 13 sowie Gonosomen) war unauffällig. Es wurde die medizinische Indikation zum Schwangerschaftsabbruch gestellt. Die Autopsie bestätigte die Befunde am Herz und im Gesicht, aufgrund der autolytischen Prozesse war eine Beurteilung des Gehirnes aber nicht mehr möglich. Zusätzlich zeigte sich eine Hufeisenniere.

In der Kultur der Amniozentese konnte durchgängig eine freie Trisomie 9 nachgewiesen werden.

Schlussfolgerung Die freie Trisomie 9 ist ein sehr seltenes Krankheitsbild. Es umfasst ein weites Spektrum an unterschiedlichen Fehlbildungen. Der vorliegende Fall liefert weitere phänotypische Details zur Trisomie 9.

\section{P3.022 Partielle Monosomie des langen Arms von Chromosom 13 - das Orbeli-Syndrom - eine Rarität}

Autoren $\underline{\text { Voigt } C^{1}}$, Beyer $]^{1}$, Lauten $A^{1}$, Schneider $U^{1}$, Schulz $S^{1}$, Fröber $R^{1}$, Schleussner $\mathrm{E}^{1}$

Institut 1 Universitätsklinikum Jena, Jena/DE

DOI 10.1055/s-0037-1607014

Problemstellung 28-jährige Patientin, Gravida I Para 0, in der 21. SSW mit fetalen Hirnfehlbildungen - dem V.a. eine Dandy-Walker-Malformation und okzipitale Encephalocele.

Patienten und Methode Die zytogenetische Untersuchung ergab einen auffälligen weiblichen Karyotyp 46,XX, del(13)(q22.1). Molekulargenetisch entspricht das einer partiellen Monosomie des langen Arms von Chromosom 13. Nach durchgeführtem perinatologischen Konsil entschied sich die Patientin zur Beendigung der Schwangerschaft nach §218a (2) aus medizinischer Indikation.

Ergebnis In der paidopathologischen Untersuchung zeigte sich ein medianer umschriebener Calvariadefekt mit interparietal gelegener zystischer Cele im Sinne einer Meningocele. Eine erhebliche Mikrocephalie, vorallem im occipitalen Bereich, eine Arhinencephalie und eine Mikrognathie bei gleichzeitiger Verbreiterung und Abflachung des Nasenrückens. Darüber hinaus wurde postmortal eine kongenitale Fehlbildung des Herzens diagnostiziert.

Schlussfolgerung Eine Kombination von Mikrocephalie und Mikrognathie ist extrem selten und geht mit einer partiellen Monosomie des langen Arms von Chromosom 13 einher. Die phänotypische Ausprägung des ,13q-Syndroms“, auch Orbeli-Syndrom genannt, ist dabei abhängig von der deletierten Chromosomenbande. Allgemein treten Retinoblastome, mentale - und Wachtumsretardierungen, Missbildungen des Gehirns, Herzdefekte, Veränderungen der distalen Gliedmaßen und auch Fehlbildungen des Urogenitaltraktes auf. Die Inzidenz der Encephalocele beträgt 1:5000 Lebendgeburten.

\section{P3.023 Sonoanatomische Befunde eines Feten mit kompletter Trisomie 22}

Autoren $\underline{\text { Voigt } C^{1}}$, Beyer $]^{1}$, Lauten $A^{1}$, Schneider $U^{1}$, Schreyer $I^{1}$, Fröber $R^{1}$, Schleussner $\mathrm{E}^{1}$

Institut 1 Universitätsklinikum Jena, Jena/DE

DOI 10.1055/s-0037-1607015

Problemstellung Eine 35-jährige Patientin, Gravida IV Para II, in der $19+2$. SSW mit einem erheblich wachstumsretardiertem Kind und dem V.a. ein syndromales Geschehen mit komplexen fetalen Fehlbildungen.

Patienten und Methode Ultrasonografisch stellte sich ein Hydrops fetalis mit Hydrothorax, Aszites und Hygroma colli, eine Dextrocardie, V.a. AVSD, LKGS, V.a. Hypertelorismus, Nierenaplasie rechts und -dysplasie links mit Anurie und fehlender Blasendarstellung sowie ein Oligohydramnion dar. Die Röhrenknochen zeigten sich ubiquitär verkürzt. Cerebral zeigte der V.a. eine komplexe Hirnfehlbildungen und eine okzipitale Encephalocele. Die zytogenetische Untersuchung ergab einen auffälligen männlichen Karyotyp 47,XY,+22. 
Ergebnis Nach durchgeführtem perinatologischen Konsil entschied sich die Patientin zur Beendigung der Schwangerschaft nach §218a (2) aus medizinischer Indikation. Der paidopathologische Befund bestätigte die pränatal erhobenen Befunde im Sinne der morphologischen Stigmata einer kompletten Trisomie 22.

Schlussfolgerung Die Trisomie 22 ist die dritthäufigste autosomale Trisomie. Sie tritt in $0,4 \%$ aller Schwangerschaften und $5 \%$ aller Spontanaborte auf. Die komplette Nichtmosaik-Form ist bei Lebendgeburten extrem selten. Herzanomalien werden bei $85 \%$ der Kinder mit kompletter Trisomie 22 beschrieben. Dabei ist, wie auch im vorliegenden Fall, das Vorherrschen atrioventrikulärer Septumdefekte zu beobachten. In 78\% treten kraniofaziale Dysmorphien auf. Malformationen des Gehirns und urogenitale Anomalien werden bei Kindern mit kompletter Trisomie 22 ebenfalls gehäuft beobachtet.

\section{P3.024 Pränataler Hydrometrokolpos, Differentialdiagnosen und assoziierte Fehlbildungen}

Autoren Mallmann $\mathrm{M}^{1}$, Reutter $\mathrm{H}^{2}$, Gottschalk $\mathrm{I}^{3}$, Mack-Detlefsen $\mathrm{B}^{4}$,

Boemers $T^{4}$, Geipel $A^{5}$, Berg $C^{6}$, Gembruch $U^{5}$

Institut 1 Uniklinik Köln, Köln/DE; 2 University of Bonn, Bonn/DE;

3 Universitätsfrauenklinik Köln, Köln/DE; 4 Kinderkrankenhaus Amsterdamer Straße Köln, Köln/DE; 5 Universitätsklinik Bonn, Bonn/DE;

6 Universitätsfrauenklinik Köln, Köln/DE

DOI 10.1055/s-0037-1607016

Problemstellung Der fetale Hydrometrokolpos ist eine seltene Malformation, welche durch eine Anhäufung von Sekret in Vagina und Uterus aufgrund einer angeborenen Obstruktion der Vagina verursacht wird. Er kann isoliert oder kombiniert mit anderen Missbildungen als Teil einer syndromischen Störung auftreten. Aufgrund der Seltenheit der Krankheit gibt es keine großen Serien, die das pränatale Spektrum der Erkrankung oder zusätzliche Fehlbildungen beschreiben.

Patienten und Methode In dieser Studie stellen wir die pränatalen und postnatalen Befunde in einer Reihe von 19 Fällen in zwei tertiären Überweisungszentren von 2004 bis 2016 vor.

Ergebnis Alle Feten zeigten ein Hydrometrokolpos. 5 Feten zeigten eine zusätzliche Hexadaktylie und wurden mit dem McKusick-Kaufman-Syndrom oder dem Bardet-Biedl-Syndrom diagnostiziert. 11 Feten zeigten Kloaken-Fehlbildungen (zusätzliche Fehlbildungen: VSD in 2 Feten, Spalthand und LPVSC in 1 Fetus). Bei 2 Feten wurde ein Mayer-Rockitansky-Küster-Hauser-Syndrom II (1 Fetus mit Agenesie der rechten Niere) diagnostiziert. 1 Fetus zeigte ein intraabdominal wachsendes zystisches Teratom mit Fistel zum Hydrometrokolpos.

Schlussfolgerung Diese Erkenntnisse veranschaulichen das Spektrum der Erkrankung in der pränatalen Diagnostik. Insbesondere eine dezidierte Differentialdiagnostik zu anderen zystischen Befunden im fetalen Abdomen ist notwendig. 


\section{Namenverzeichnis}

\author{
A \\ Akengin A S3, S43 \\ Alk A S27 \\ Al-Nawas B S25 \\ Altmann R S11, S14 \\ Ammann S S30 \\ Apitz C S37 \\ Arabin B S17, S53 \\ Arampatzis S S51 \\ Arzt W S11, S14 \\ Ausmeier A S7, S48 \\ Ausserer C S34 \\ Axt-Fliedner R S8, S9, S10, S21, S58
}

B

Bahlmann H S36

Baier F S16, S53

Balzer AK S23

Bamberg C $\mathrm{S} 10$

Bamberger CM $\$ 47$

Barbara G $\$ 49$

Barkhaus C S53

Bauermeister W S28, S29, S50

Baumann T S35, S49

Becker M S45

Becker R S59

Becker WH S54, S61

Beckmann M S16, S18, S21, S53

Beerbaum P S37

Behrens CB S37

Berg C S9, S13, S62

Bergner R S25

Beyer J S58, S59, S61

Bibra $\mathrm{H}$ von $\mathrm{S} 31$

Bingisser R S30

Birdir C S18, S54

Blank W S47

Bleif M S45

Boemers T S62

Bohnhorst B S37

Boozari B S3, S43

Bosselmann S S8, S20, S55

Braun B 547

Braun J S14, S15, S22

Breuer J S32

Brückmann A $\$ 19$

Brückmann D $\quad$ S19

Brünn K S3

Brunner C S51

Brunner H S37

Burkhard F S51

Burkhardt H S43

Busack B S13

C

Caca K S4

Carbon R S21

Chupina V S6

Claussen J $\mathbf{S 4 0}$

Crispi F S10

Czihal M S7

$$
\text { D }
$$

Dähne F $\quad$ S48

Darkwah Oppong M S19

de Catte L S8

de Gregorio A S42

de Gregorio N S42

de Vries $\mathrm{H} \quad \mathrm{S} 17$

Dechant C S7

Dechant FX S3

Degenhardt J S8, S9

Delius M S16

Diederich H S43

Diemon N S14
Dietrich CF S45

Dirks K S3

Dobler F S50

Dombrowski F S52

Dörffel Y S23

Dörnemann R S17

Dracopoulos C S57

Droste L S18

Duwe W S52

E

Eberhardt O S28

Ebner F S17, S42

Eggensberger T S59

Eichhorn KH S17

Eichler H S21

Eixarch E S10, S15

Entezami M S59

Enzensberger C S8, S9, S10, S21, S58

Erker C S51

Essig S S35, S49

Eveslage M S15

Faber R S11, S12, S53, S55

Falkenberg M S10, S15, S17

Faschingbauer F S16, S18, S21, S53

Feldkamp A S36

Fernandes T S32, S57

Fernandez Sanchez J S27, S47, S61

Festl J S51

Figiel J S34

Findeisen H S34

Flemmer A S16

Forster B S29

Forster I S29

Fox L S18

Frank M S18

Franke D S23

Friebe B S7

Friebe-Hoffmann U S12, S17, S42

Friedl T S12, S17

Fröber R S17, S59, S61

Fröhlich C S51

Fröhlich E S4, S34, S46

Fruscalzo A S15

Funke K S14

Füssinger MA S26

\section{G}

Gass M S26

Gehlen M S28

Gehmacher O S34

Geiler S S11, S12, S53, S55

Geipel A S13, S62

Gelbrich T S3

Gellhaus A S18

Gembicki M S6, S32, S57, S60

Gembruch U S13, S62

Gittinger FS S2

Gloning KP S9, S56

Glosemeyer P S10

Goetze E] S25

Görg C S5, S34, S42, S43, S44, S45

Görg K S34

Görtz R S22

Götte M S21, S58

Gottschalk I S62

Gratacós E S10, S15

Gräter T S4, S5, S50

Graupner O S10, S16

Greene B S34

Greger A S44

Grotz A S4, S34, S46

Grüner B S5, S50
Guth S S47

Guthoff AE S47

$\mathrm{H}$

Hafner E S18

Hahn G S38

Halloul Z S7

Hammer K S14, S15

Hammer M S28

Hammer R S12

Hamoud BH S21

Hamza A S14, S21, S49, S53

Hänle M S4, S5, S50

Hartge D S6, S32, S57, S60

Harth S S37

Hasbargen U S16

Hawemann M S7

Hechenberger C S11

Hecher K S10

Heckmann M S36

Heese S S22

Henrich W S9, S13, S57, S59

Herberg U S32

Herrmann J S9, S58

Heverhagen J S51

Hiltmann A S12

Hodul A S41

Hofauer B S25, S26

Hoffmann U S7

Hönig DK S17

Hübener C S16

Huhle D S55

Hummler H S37

Hütter W S17

(a)

lannaccone A S19

Ignee A S45

Ittermann T S36

$\sqrt{2}+x^{2}$

Jahn C S58

Janke A S9, S56

Janni W S12, S42

Juhasz-Boess I S53

Jung EM S3

K

Käb A S45

Kaisenberg $C$ von S37

Kalache K S57, S59

Kalle T von S28

Kallenbach M S23

Kämmer JC S6

Kawecki A S8, S9

Kehl S S16, S21, S53

Keilmann N S41

Khalil M S10

Kiener TA S14

Kimmig R S18, S19

Kirby N S18

Kirchner Gl S3

Kiss B S51

Klee D S31

Klinger C S4, S34, S46

Klockenbusch W S10, S14, S15, S17

Knieling F S40

Knopf A S25, S26

Koch R S17

Köhler S S21

Kollmann C S36, S41

Koluch A S37

Komar M S54

Konermann M S44 
Köninger A S18, S19

Kossow A S22

Kozak J S27

Kozlowski P S12

Krähenbühl G S30

Krapp M S8, S61

Kratzer W S4, S5, S50

Krause K S10

Kreiselmaier $\mathrm{P} \quad \mathrm{S} 61$

Krombach GA S37

Kropf S S7

Kropp AT S47

Kruse A S4, S34, S46

Kubicka S S3, S43, S47

Kurkevych A S8

Kyvernitakis I S17

$$
\text { L. }
$$

Lachmann R S19

Lange A S36

Lange L S42

Lato C S42

Lato $\mathrm{K}$ S12, S17, S42

Lauten A S58, S59, S61

Lebek H S9

Lechleitner $\mathrm{P} \quad \mathrm{S46}$

Leclerque C S31

Lees C S16

Linden K S32

Lobmaier S S10, S15, S16

Löffler C S25

Lottspeich C S7

Luths A S54

Lützow C S4

M

Mack-Detlefsen B S62

Mahner S S16

Maier A S28

Malek M S36

Malek NP S3, S43

Mallmann M S62

Mamalis M S8

Mansour N S25, S26

Marinova M S4

Martin T S27

Martinez J S10, S15

Mathis G S33

Maxeiner S S53

Mayr A S16, S53

Meier CM S14

Meiss AL S47

Mentzel HJ S17

Merkel D S6

Merz E S19

Meuter A S16

Meyberg-Solomayer GC S14, S21, S49, S53

Meyer F S7

Meyer-Wittkopf M S8

Micheletti T S15

Mielke G S8, S20, S55

Minderer S S9, S11

Miric Tesanic D S19

Misselwitz B S53

Moergel M S25

Möller A S17

Möllers M S10, S14, S15, S17, S22

Möllmann M S51

Möllmann U S17

Mommsen H S11, S56

Morhart P S21

Mühlhaus K S37

Müller A S16

Müller AM S11

Müller T S47

Müller V S14

Munkhuu B S35

Munkuu B S35, S49
N

Nasko M S28

Neesse A S43, S44

Neubauer A S5

Neurath M S22, S40

Nieves Ortega R S30

Nitsch T S27

Nitzsche K S54

Noever K S53

Nonnenmann E S17

Nurkan E S37

0

Oelmeier de Murcia K S15, S22

Oesterwind KA S29

Ortiz J S10, S15, S16

Oturina V S15

P

Paulick J S9

Peter D S37

Petz V S24

Pfahler M S4, S50

Pfeifer L S22

Pilch J S21

Polasik A S42

Pretscher J S16, S21, S53

Pschebezin F S14

Püttmann S S4

$$
\mathrm{R}
$$

Raabe E S16, S53

Radosa J S53

Rascher W S40

Raßmann P S28, S29

Recker F S32

Rehn M S51

Reifeltshammer C S33

Respondek-Liberska M S8

Reutter H S62

Riecken B S4, S34, S46

Ripoll C S2

Riße S S11, S12, S53, S55

Ritgen J S8, S9

Rizzo G S8

Rosenbaum T S36

Rostock L S58

Ruhland F S52

$$
\text { S }
$$

Santamaria M S51

Sattler H S25

Sauerland C S14

Schaper」 S31

Scharnreitner I S11, S14

Scheel M S28

Scheidt $F$ von S37

Scheier M S11

Scheiermann P $\$ 49$

Schellhaas B S22

Schicht C S27

Schild R S37, S53

Schilling U S19

Schimke C S14

Schlembach D S58

Schleußner E S58, S59

Schleussner E S61

Schmehl I S48

Schmelzeisen R S26

Schmid M S16, S53

Schmid R S35, S49

Schmidberger J S4, S5, S50

Schmidt B S18

Schmidt G $\$ 16$

Schmitz J S22

Schmitz R S10, S14, S15, S17, S22

Schneider C S6

Schneider H S18
Schneider M S16, S53

Schneider P S18

Schneider U S17, S58, S59, S61

Schochter F S42

Schöll E S29, S30

Schramm T S9, S11, S56

Schreiber S S51

Schreyer I S61

Schröder W S45

Schröttle A S7

Schubert C S37

Schuessler G S45

Schuler A S45, S47

Schulz S S17, S61

Schulze W S9

Schulze-Koops H S7

Schwärzler B S34

Schwerdtfeger R S37

Seeger M S24

Seitz G S21

Semper-Hogg W S26

Sengewein J S36

Shoukier M S56

Siegmund T S31

Sklavounos P S21

Słodki M S8

Sohlbach K S45

Solomayer EF S21, S53

Specht C S11

Springer C S14

Staboulidou I S37

Stark K S49

Steinhard J S14

Stepan H S11, S12, S53

Stressig R S8, S9

Strizek B S13

Strobel D S22

Ströder R S53

Strunk H S4

Stumpfe F S16, S21

Stumpfe FM S53

Südhoff T S27

T

Takacs FZ S14, S49

Takacs Z S21, S53

Taut H S38

Thieme-Ruffing S S49

Thomas V S39

Thome UH S55

ThulJ S8, S9

Tiefenbach M S28

Timmesfeld N S53

Tinschert S S8

Tomasi R S49

Torres X S10, S15

Tramontana AA S18

Trenker C S5, S34, S42, S43, S44, S45

Tschernig T S53

Tshering Vogel D S51

Tucher E von S9, S57, S59

$\mathrm{U}$

Udelnow A S7

Urban P S8

V

Verlohren S S13

Vilser C S17

Vogeler $\mathrm{F}$ S52

Vogt B S51

Voigt C S61

Vosko MR S51

W

Wadsack D S25

Waldner M S40

Weichert A S9, S57, S59 
Weichert J S6, S32, S57, S60

Weise J S36

Wermke W S23

Wertaschnigg D S14

Weskott HP S7, S48

Wieg L S10

Wilczynski A $\$ 42$

Will U S43

Wimberger $\mathrm{P} \quad \mathrm{S} 18$

Winkler C S32

Wirth T S28
Wöckel A S51

Wohlfart S S18

Wohlmuth C S14

Wollmer E S5

Wolter A S8, S9, S10, S58

Wowra T S37

Wüstemann M S37

Yerebakan C S10

Yu Y S31
Zemlin M S14

Zettinig G S24

Zierl A S20, S28

Zimmermann P 543

Zych-Krekora K S8 